\title{
Euler characteristics of Teichmüller curves in genus two
}

\author{
MATT BAINBRIDGE
}

We calculate the Euler characteristics of all of the Teichmüller curves in the moduli space of genus two Riemann surfaces which are generated by holomorphic one-forms with a single double zero. These curves are naturally embedded in Hilbert modular surfaces and our main result is that the Euler characteristic of a Teichmüller curve is proportional to the Euler characteristic of the Hilbert modular surface on which it lies.

The idea is to use techniques from algebraic geometry to calculate the fundamental classes of these Teichmüller curves in certain compactifications of the Hilbert modular surfaces. This is done by defining meromorphic sections of line bundles over Hilbert modular surfaces which vanish along these Teichmüller curves.

We apply these results to calculate the Siegel-Veech constants for counting closed billiards paths in certain L-shaped polygons. We also calculate the Lyapunov exponents of the Kontsevich-Zorich cocycle for any ergodic, $\mathrm{SL}_{2}(\mathbb{R})$-invariant measure on the moduli space of Abelian differentials in genus two (previously calculated in unpublished work of Kontsevich and Zorich).

32G15, 37D50; 11F41, 30F30

\section{Introduction}

Let $\mathcal{M}_{2}$ be the moduli space of genus two Riemann surfaces. Given any $D>0$ with $D \equiv 0$ or $1(\bmod 4)$, let $W_{D}^{\prime} \subset \mathcal{M}_{2}$ be the locus of Riemann surfaces $X$ such that:

- The Jacobian $\operatorname{Jac}(X)$ has real multiplication by $\mathcal{O}_{D}$, the unique real quadratic order of discriminant $D$.

- There is an Abelian differential $\omega$ on $X$ which is an eigenform for real multiplication and has a double zero.

Let $W_{D}$ be the normalization of $W_{D}^{\prime}$, a possibly disconnected curve.

The Hilbert modular surface,

$$
X_{D}=\mathbb{M} \times \mathbb{M} / \operatorname{SL}\left(\mathcal{O}_{D} \oplus \mathcal{O}_{D}^{\vee}\right)
$$


is the moduli space for Abelian surfaces with real multiplication by $\mathcal{O}_{D}$. In $X_{D}$, let $P_{D}$ be the Shimura curve consisting of those Abelian surfaces in $X_{D}$ which are polarized products of elliptic curves. There is a commutative diagram,

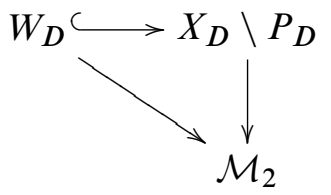

where the top map is an embedding, and the vertical map is a two-to-one map sending an Abelian surface $A$ to the unique Riemann surface $X \in \mathcal{M}_{2}$ such that $\operatorname{Jac}(X) \cong A$.

The curve $W_{D}$ is not a Shimura curve on $X_{D}$; however, it is a Teichmüller curve, a curve which is isometrically immersed in the moduli space $\mathcal{M}_{2}$ with respect to the Teichmüller metric. In fact, the curves $W_{D}$ with $D$ nonsquare are all but one of the primitive Teichmüller curves in $\mathcal{M}_{2}$, where a Teichmüller curve is said to be primitive if it does not arise from a Teichmüller curve of lower genus by a certain branched covering construction. The curves $W_{D}$ arise from the study of billiards in certain $L$-shaped polygons, and the study of these curves has applications to the dynamics of billiards in these polygons.

Euler characteristics The main object of this paper is to calculate the Euler characteristics of the curves $W_{D}$. The idea is to relate $\chi\left(W_{D}\right)$ to $\chi\left(X_{D}\right)$ and $\chi\left(P_{D}\right)$. It turns out that these Euler characteristics are all proportional if $D$ is not square. The following is our main result.

Theorem 1.1 If $D$ is not square, then

$$
\chi\left(W_{D}\right)=-\frac{9}{2} \chi\left(X_{D}\right) .
$$

Remark Zagier conjectured formula (1-1) from numerical evidence. In [73], Siegel calculated the volume of $X_{D}$ when $D$ is a fundamental discriminant (that is, $D$ is not of the form $D=f^{2} E$ for some $E \equiv 0$ or $1(\bmod 4)$ and $\left.f>1\right)$. This yields formulas for $\chi\left(X_{D}\right)$ for all $D$. For $D \neq 1$ a fundamental discriminant, we obtain

$$
\chi\left(W_{f^{2} D}\right)=-9 \zeta_{\mathbb{Q}(\sqrt{D})}(-1) f^{3} \sum_{r \mid f}\left(\frac{D}{r}\right) \frac{\mu(r)}{r^{2}},
$$

where $\mu$ is the Möbius function, and $\left(\frac{D}{r}\right)$ is the Kronecker symbol. When $f=1$, this reduces to

$$
\chi\left(W_{D}\right)=-9 \zeta_{\mathbb{Q}(\sqrt{D})}(-1)
$$


The Euler characteristics $\chi\left(W_{D}\right)$ when $D$ is not square are given by the Fourier coefficients of a modular form. More precisely, there is a function $H(2, D)$ such that for $D \neq 1$ a fundamental discriminant and $f \in \mathbb{N}$,

$$
\sum_{r \mid f} \chi\left(W_{r^{2} D}\right)=\frac{3}{4} H\left(2, f^{2} D\right)
$$

and

$$
\sum_{\substack{D \equiv 0,1(4) \\ D \geq 0}} H(2, D) q^{D}
$$

is a modular form which was studied by Cohen in [19]. See Section 2.3 for more about this form.

When $D=d^{2}$, Eskin, Masur, and Schmoll calculated $\chi\left(W_{d^{2}}\right)$ in [22]. Our methods give a new proof of their formula. In this case, it is no longer true that $\chi\left(W_{d^{2}}\right)$ is proportional to $\chi\left(X_{d^{2}}\right)$.

Theorem 1.2 For any $d>1$,

$$
\chi\left(W_{d^{2}}\right)=-\frac{1}{16} d^{2}(d-2) \sum_{r \mid d} \frac{\mu(r)}{r^{2}} .
$$

Connected components of $\boldsymbol{W}_{\boldsymbol{D}}$ McMullen showed that when $D \neq 9$ and $D \equiv$ $1(\bmod 8), W_{D}$ has two connected components, $W_{D}^{0}$ and $W_{D}^{1}$; otherwise $W_{D}$ is connected [54].

We will also show that when $D$ is not square, the connected components of $W_{D}$ have the same Euler characteristic. It should be true that these components are actually homeomorphic, but we have not proved this. It is not true that the connected components of $W_{D}$ are isomorphic as curves over $\mathbb{C}$.

Theorem 1.3 If $D \equiv 1(\bmod 8)$ is not square, then

$$
\chi\left(W_{D}^{0}\right)=\chi\left(W_{D}^{1}\right)
$$

There is a canonical involution $\tau: X_{D} \rightarrow X_{D}$ which is covered by the involution $(z, w) \mapsto(w, z)$ of $\mathbb{M} \times \mathbb{H}$. This involution replaces an Abelian surface $A$ together with a choice of real multiplication,

$$
\rho: \mathcal{O}_{D} \rightarrow \text { End } A,
$$


with the Galois conjugate real multiplication $\rho^{\prime}$ obtained by precomposing $\rho$ with the Galois automorphism of $\mathcal{O}_{D}$. It is not true that $\tau$ permutes the connected components of $W_{D}$ (otherwise Theorem 1.3 would be trivial). In fact, $W_{D}$ is not even invariant under $\tau$ by [52, Theorem 10.1].

We also calculate $\chi\left(W_{d^{2}}^{\epsilon}\right)$, but it is no longer true in the square discriminant case that the connected components of $W_{d^{2}}$ have the same Euler characteristic.

Theorem 1.4 If $d>0$ and $d^{2} \equiv 1(\bmod 8)$, then

and

$$
\begin{aligned}
& \chi\left(W_{d^{2}}^{0}\right)=-\frac{1}{32} d^{2}(d-1) \sum_{r \mid d} \frac{\mu(r)}{r^{2}}, \\
& \chi\left(W_{d^{2}}^{1}\right)=-\frac{1}{32} d^{2}(d-3) \sum_{r \mid d} \frac{\mu(r)}{r^{2}} .
\end{aligned}
$$

Remark These formulas were established independently by Lelièvre and Royer [47].

Siegel-Veech constants Given a positive, nonsquare integer $D \equiv 0$ or $1(\bmod 4)$, let $P(D)$ be the L-shaped polygon as in Figure 1, where the side lengths are given by

$$
a=b=\frac{1+\sqrt{D}}{2}
$$

if $D$ is odd and by

$$
a=\frac{\sqrt{D}}{2} \quad \text { and } \quad b=1+\frac{\sqrt{D}}{2}
$$

if $D$ is even. By a standard unfolding construction, an $L$-shaped polygon determines a genus two Riemann surface equipped with an Abelian differential, and the surface determined by $P(D)$ lies on $W_{D}$.

A billiards path on $P(D)$ is a path which is geodesic on the interior of $P(D)$ and bounces off the walls as a physical billiards ball would (angle of incidence equals angle of reflection). We allow billiards paths to pass through the corners of angle $\pi / 2$ but not through the one of angle $3 \pi / 2$. Closed billiards paths occur in parallel families which are bounded by two paths which each start and end at the corner of angle $3 \pi / 2$. Let

$N(P(D), L)=\#\{$ families of simple closed billiards paths on $P(D)$

of length at most $L\}$. 


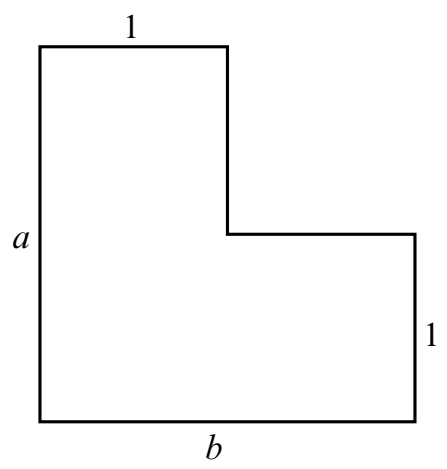

Figure 1: $P(D)$

Note that we do not count paths which go around a single closed path several times, and we are counting unoriented paths. Since the unfolding lies on a Teichmüller curve, we have by [77]

$$
N(P(D), L) \sim c(D) \frac{1}{8 \pi \operatorname{Area}(P(D))} L^{2},
$$

for some constants $c(D)$ called Siegel-Veech constants. Evaluating the constants $c(D)$ amounts to calculating the Euler characteristics of the $W_{D}$ together with the volumes of certain neighborhoods of the cusps of $W_{D}$. In Section 14, we calculate these constants. For example, we obtain:

Theorem 1.5 For $D \leq 29$, the Siegel-Veech constants $c(D)$ are as given in Table 1 .

\begin{tabular}{|l|cccccccccc|}
\hline$D$ & 5 & 8 & 12 & 13 & 17 & 20 & 21 & 24 & 28 & 29 \\
\hline$c(D)$ & $\frac{25}{3}$ & $\frac{28}{3}$ & $\frac{26}{3}$ & $\frac{91}{9}$ & $\frac{221}{24}+\frac{1}{8} \sqrt{17}$ & $\frac{31}{3}$ & $\frac{133}{15}$ & $\frac{148}{15}$ & $\frac{82}{9}$ & $\frac{377}{35}$ \\
\hline
\end{tabular}

Table 1: Siegel-Veech constants for $P(D)$

Note that there are other L-shaped polygons, classified in [52], whose unfoldings lie on the Teichmüller curves $W_{D}$ and our results apply equally well to those because the Siegel-Veech constants only depend on the connected component of $W_{D}$ on which the unfolding lies. We also show in Section 14:

Theorem 1.6 If $W_{D}$ has two connected components, then the associated Siegel-Veech constants are Galois-conjugate elements of $\mathbb{Q}(\sqrt{D})$. If $W_{D}$ is connected, then the Siegel-Veech constants are rational. 
The Kontsevich-Zorich cocycle There is a well-known action of $\mathrm{SL}_{2} \mathbb{R}$ on $\Omega \mathcal{M}_{g}$, the moduli space of genus $-g$ Riemann surfaces equipped with an Abelian differential, which preserves the subspace $\Omega_{1} \mathcal{M}_{g}$ of Abelian differentials $(X, \omega)$ of unit norm with respect to the norm

$$
\|\omega\|=\left(\int_{X}|\omega|^{2}\right)^{1 / 2} .
$$

The diagonal subgroup $A \subset \mathrm{SL}_{2} \mathbb{R}$ induces a flow $g_{t}$ on $\Omega \mathcal{M}_{g}$, the Teichmüller geodesic flow. Kontsevich [42] and Zorich [84] introduced a linear cocycle over this flow which is closely related to the dynamics of flows on surfaces. There is a bundle $\mathcal{H}_{1}(\mathbb{R}) \rightarrow \Omega_{1} \mathcal{M}_{g}$ whose fiber over an Abelian differential $(X, \omega)$ is the quotient $H_{1}(X ; \mathbb{R}) / \operatorname{Aut}(X, \omega)$. This bundle has a natural flat connection, the Gauss-Manin connection. By coupling the geodesic flow $g_{t}$ with the Gauss-Manin connection, we get a flow $\tilde{g}_{t}$, the Kontsevich-Zorich cocycle, on $\mathcal{H}_{1}(\mathbb{R})$ which is linear on the fibers and covers the flow $g_{t}$ on $\Omega_{1} \mathcal{M}_{g}$.

Consider the closure $S \subset \Omega_{1} \mathcal{M}_{2}$ of an $\mathrm{SL}_{2} \mathbb{R}$ orbit. By results of [57], $S$ is a suborbifold of $\Omega_{1} \mathcal{M}_{2}$ which comes equipped with a unique $\mathrm{SL}_{2} \mathbb{R}$-invariant, ergodic, absolutely continuous, probability measure, the period measure. In the case of the Teichmüller curves, period measure is just the normalized hyperbolic area measure (because Haar measure is unique).

Associated to any ergodic, $g_{t}$-invariant measure $\mu$ are the $2 g$ Lyapunov exponents,

$$
1=\lambda_{1}(\mu)>\cdots>\lambda_{g}(\mu)>-\lambda_{g}(\mu)>\cdots>-\lambda_{1}(\mu)=-1 .
$$

Kontsevich [42] gave a formula for $\sum \lambda_{i}(\mu)$ as a certain integral over moduli space when $\mu$ is $\mathrm{SL}_{2} \mathbb{R}$ invariant. The same methods which we use to calculate $\chi\left(W_{D}\right)$ allow us to evaluate these integrals and to calculate $\lambda_{2}(\mu)$ for all ergodic, $\mathrm{SL}_{2} \mathbb{R}$-invariant measures on $\Omega_{1} \mathcal{M}_{2}$. Let $\Omega_{1} \mathcal{M}_{2}(2) \subset \Omega_{1} \mathcal{M}_{2}$ be the locus of forms with a double zero.

Theorem 1.7 If $\mu$ is any finite, ergodic, $\mathrm{SL}_{2} \mathbb{R}$-invariant measure on $\Omega_{1} \mathcal{M}_{2}$, then

$$
\lambda_{2}(\mu)= \begin{cases}1 / 3, & \text { if } \mu \text { is supported on } \Omega_{1} \mathcal{M}_{2}(2) \\ 1 / 2, & \text { if } \mu \text { is not supported on } \Omega_{1} \mathcal{M}_{2}(2)\end{cases}
$$

Remark Theorem 1.7 is an unpublished result of Kontsevich and Zorich, mentioned in [85]. 
Outline of proof of Theorem 1.1 The strategy for computing the Euler characteristic of $W_{D}$ is to study the relationship between $W_{D}$ and the Hilbert modular surface $X_{D}$ on which it lies. More precisely, we will define a compactification $Y_{D}$ of $X_{D}$ and compute the fundamental class of $\bar{W}_{D}$ in $Y_{D}$ by expressing it as the zero locus of a meromorphic section of a line bundle over $Y_{D}$.

Here is a sketch of the calculation of the Euler characteristic $\chi\left(W_{D}\right)$ in the case when $D$ is nonsquare. When $D$ is a square, the calculation is more complicated because there are some extra curves in $Y_{d^{2}} \backslash X_{d^{2}}$ which have to be taken into account.

(1) Given a nonsquare $D \in \mathbb{N}$ with $D \equiv 0$ or $1(\bmod 4)$, let

$$
\mathcal{O}_{D}=\mathbb{Z}[T] /\left(T^{2}+b T+c\right)
$$

for integers $b$ and $c$ such that $b^{2}-4 c=D$. The discriminant $D$ determines $\mathcal{O}_{D}$ up to isomorphism, and $\mathcal{O}_{D}$ is naturally embedded in its field of fractions $K_{D}$, which is isomorphic to $\mathbb{Q}(\sqrt{D})$. Let $\mathcal{O}_{D}^{\vee} \subset K_{D}$ be the inverse different of $\mathcal{O}_{D}$, and let $\operatorname{SL}\left(\mathcal{O}_{D} \oplus \mathcal{O}_{D}^{\vee}\right) \subset \mathrm{SL}_{2} K_{D}$ be the subgroup which preserves $\mathcal{O}_{D} \oplus \mathcal{O}_{D}^{\vee}$. There are two closed two-forms $\omega_{i}$ on

$$
X_{D}=\mathbb{H} \times \mathbb{H} \operatorname{SL}\left(\mathcal{O}_{D} \oplus \mathcal{O}_{D}^{\vee}\right)
$$

covered by the forms

$$
\frac{1}{2 \pi} \frac{d x_{i} \wedge d y_{i}}{y_{i}^{2}}
$$

on $\mathbb{M} \times \mathbb{M}$. The inverse image of $W_{D}$ in $\mathbb{W} \times \mathbb{W}$ is the union of the graphs of countably many holomorphic functions $\mathbb{H} \rightarrow \mathbb{H}$. It follows that the restriction of $\omega_{1}$ to $W_{D}$ is $1 / 2 \pi$ times the hyperbolic volume form on $W_{D}$. Gauss-Bonnet then tells us that

$$
\chi\left(W_{D}\right)=-\int_{W_{D}} \omega_{1}
$$

(2) There is a Shimura curve $P_{D} \subset X_{D}$ which parameterizes Abelian surfaces which are polarized products of elliptic curves. It is covered in $\tilde{X}_{D}$ by a union of graphs of Möbius transformations $\mathbb{H} \rightarrow \mathbb{H}$. We introduce these curves in Section 2.3 and show that

$$
\chi\left(P_{D}\right)=-\frac{5}{2} \chi\left(X_{D}\right)
$$

(3) The bundle $\Omega \mathcal{M}_{2}$ over $\mathcal{M}_{2}$ whose fiber over a Riemann surface $X$ is the space $\Omega(X) \backslash\{0\}$ of nonzero Abelian differentials on $X$ extends to a bundle $\Omega \overline{\mathcal{M}}_{2}$ over the Deligne-Mumford compactification which we discuss in Section 5. In Section 7 and Section 8, we construct a compactification $Y_{D}$ of $X_{D}$, a complex 
projective orbifold which is obtained by taking the normalization of the closure of the image of an embedding $X_{D} \rightarrow \mathbb{P} \Omega \overline{\mathcal{M}}_{2}$. We call this the geometric compactification of $X_{D}$. The complement $\partial X_{D}=Y_{D} \backslash X_{D}$ consists of finitely many rational curves, each labeled by a discrete invariant which we call a $Y_{D^{-}}$ prototype. We discuss these invariants in Section 3. For each $Y_{D}$-prototype $P$, there is one rational curve $C_{P} \subset \partial X_{D}$.

(4) Associated to each point $p \in Y_{D}$, there is a stable Riemann surface $X \in \overline{\mathcal{M}}_{2}$ together with an action of $\mathcal{O}_{D}$ on $\operatorname{Jac}(X)$ by real multiplication. This determines a splitting of the space $\Omega(X)$ of stable Abelian differentials on $X$ into two onedimensional eigenspaces for real multiplication:

$$
\Omega(X)=\Omega^{1}(X) \oplus \Omega^{2}(X) .
$$

(See Section 5 for information about stable Riemann surfaces and stable Abelian differentials.) A choice of an embedding $\mathcal{O}_{D} \rightarrow \mathbb{R}$ determines one of these two eigenspaces, so the point $p \in Y_{D}$ determines an eigenform in $\Omega(X)$ up to scale if we fix such an embedding. There is a holomorphic line bundle $\Omega Y_{D}$ whose fiber over $p \in Y_{D}$ is the eigenspace $\Omega^{1}(X)$.

In Section 9, we study a holomorphic foliation $\mathcal{A}_{D}$ of $Y_{D}$ along whose leaves the absolute periods of eigenforms in $\Omega Y_{D}$ are locally constant. Each rational curve $C_{P} \subset \partial X_{D}$ is a leaf of $\mathcal{A}_{D}$, and $\mathcal{A}_{D}$ has isolated singularities at the intersection of two such curves. A leaf of $\mathcal{A}_{D}$ can be locally parameterized away from $W_{D}$ and $P_{D}$ by the relative periods of the forms which it represents (a relative period of a one-form $\omega$ is an integral along a path joining the two zeros $\omega$ ). Following [58], we use this parameterization to define a quadratic differential on each leaf of $\mathcal{A}_{D}$. These quadratic differentials can be pieced together to define a meromorphic section $q$ of the line bundle

$$
\mathcal{L}=\left(\Omega Y_{D}\right)^{-2} \otimes\left(T^{*} \mathcal{A}_{D}\right)^{2}
$$

over $Y_{D}$. This section $q$ vanishes along $\bar{W}_{D}$, has a simple pole along $\bar{P}_{D}$, and is elsewhere finite and nonzero. This gives a relation between the fundamental classes of $\bar{W}_{D}$ and $\bar{P}_{D}$ and the Chern class of $\mathcal{L}$ in $H^{2}\left(Y_{D} ; \mathbb{Q}\right)$ :

$$
\left[\bar{W}_{D}\right]=\left[\bar{P}_{D}\right]+c_{1}(\mathcal{L}) .
$$

We derive this formula in Section 10. This is the main idea of the calculation of $\chi\left(W_{D}\right)$, and the reader who only wants the gist of the argument might find it useful to skip directly to this section.

(5) In Section 9, we calculate $c_{1}(\mathcal{L})$. We define a Hermitian metric $h$ on $\Omega Y_{D}$ which is singular along $\partial X_{D}$. We show that $h$ is a good metric in the sense 
of Mumford [63]. This implies that the Chern form $c_{1}\left(\Omega Y_{D}, h\right)=\omega_{1} / 2$ is a closed current on $Y_{D}$, and

$$
c_{1}\left(\Omega Y_{D}\right)=\frac{1}{2}\left[\omega_{1}\right]
$$

in $H^{2}\left(Y_{D} ; \mathbb{Q}\right)$.

The canonical involution $\tau$ of $X_{D}$ extends to an involution of $Y_{D}$, which we continue to call $\tau$. We also show that

$$
\tau^{*}\left(\Omega Y_{D}\right)^{2}=T^{*} \mathcal{A}_{D},
$$

which implies

$$
c_{1}\left(T^{*} \mathcal{A}_{D}\right)=\left[\omega_{2}\right] .
$$

Putting together (1-5) and (1-6), we obtain

$$
c_{1}(\mathcal{L})=-\left[\omega_{1}\right]+2\left[\omega_{2}\right] .
$$

(6) Combining (1-4) and (1-7), we obtain

$$
\left[\bar{W}_{D}\right]=\left[\bar{P}_{D}\right]-\left[\omega_{1}\right]+2\left[\omega_{2}\right] .
$$

By pairing $-\left[\omega_{1}\right]$ with both sides and applying the Gauss-Bonnet Theorem, we obtain

$$
\chi\left(W_{D}\right)=\chi\left(P_{D}\right)-2 \chi\left(X_{D}\right) .
$$

Putting (1-3) in (1-9), we obtain (1-1).

The proof of Theorem 1.1 uses very little about the compactification $Y_{D}$. Equation $(1-8)$ is true, when interpreted as an equation of cohomology classes of closed currents on $X_{D}$; however, we are not allowed to pair $\omega_{1}$ with this equation because $\omega_{1}$ is not compactly supported. Working in the compactification $Y_{D}$ allows us to justify this paring. The proof of Theorem 1.3 uses the compactification $Y_{D}$ in a more essential way.

When $D=d^{2}$, there are some extra curves $S_{d^{2}}^{1}$ and $S_{d^{2}}^{2}$ in $Y_{d^{2}} \backslash X_{d^{2}}$, which complicates the calculation of $\chi\left(W_{d^{2}}\right)$. One difference in the square case is that the section $q$ of $\mathcal{L}$ has a pole along $\bar{S}_{d^{2}}^{2}$ as well as $\bar{P}_{d^{2}}$. Also, (1-6) becomes

$$
c_{1}\left(T^{*} \mathcal{A}_{D}\right)=\left[\omega_{2}\right]-\left[\bar{S}_{d^{2}}^{2}\right]
$$

Instead of (1-9), we get

$$
\chi\left(W_{d^{2}}\right)=\chi\left(P_{d^{2}}\right)-2 \chi\left(X_{d^{2}}\right)-\chi\left(S_{d^{2}}^{2}\right) .
$$

In this case, $\chi\left(P_{d^{2}}\right)$ and $\chi\left(S_{d^{2}}^{2}\right)$ are not proportional to $\chi\left(X_{d^{2}}\right)$, so neither is $\chi\left(W_{d^{2}}\right)$. 
Outline of proof of Theorem 1.3 The idea of the proof of Theorem 1.3 is to find as many equations as we can involving the fundamental classes $\left[\bar{W}_{D}^{0}\right]$ and $\left[\bar{W}_{D}^{1}\right]$. Once we have enough equations, we are able to solve for the pairing $\left[\omega_{1}\right] \cdot\left[\bar{W}_{D}^{\epsilon}\right]=-\chi\left(W_{D}^{\epsilon}\right)$.

(1) In Section 7 and Section 8, we will prove the following properties of $Y_{D}$ :

(a) The closures $\bar{W}_{D}$ and $\bar{P}_{D}$ are disjoint suborbifolds of $Y_{D}$.

(b) Each rational curve $C_{P} \subset Y_{D} \backslash X_{D}$ meets $\bar{W}_{D}$ and $\bar{P}_{D}$ transversely and meets each in the same number of points.

(c) The canonical involution $\tau$ of $Y_{D}$ has the property that for each rational curve $C_{P} \subset \partial X_{D}$,

$$
\bar{W}_{D}^{0} \cdot C_{P}=\bar{W}_{D}^{1} \cdot \tau\left(C_{P}\right)
$$

(d) The rational cohomology of $Y_{D}$ is an orthogonal direct sum:

$$
H^{2}\left(Y_{D} ; \mathbb{Q}\right) \cong B \oplus\left\langle\left[\omega_{1}\right],\left[\omega_{2}\right]\right\rangle \oplus J,
$$

where $B$ is the subspace generated by the rational curves $C \subset \partial X_{D}$, and $J$ is the orthogonal complement of the other two terms. $J$ contains all of $H^{2,0}\left(Y_{D} ; \mathbb{Q}\right)$ and $H^{0,2}\left(Y_{D} ; \mathbb{Q}\right)$ and most of $H^{1,1}\left(Y_{D} ; \mathbb{Q}\right)$.

(2) There is a well-known formula relating the first Chern class of the bundle $\Omega \overline{\mathcal{M}}_{2}$ over $\overline{\mathcal{M}}_{2}$ to the fundamental classes of divisors in $\overline{\mathcal{M}}_{2} \backslash \mathcal{M}_{2}$. Pulling back this formula by the natural map $Y_{D} \rightarrow \overline{\mathcal{M}}_{2}$, we obtain a formula for the fundamental class of $\left[\bar{P}_{D}\right]$ :

$$
\left[\bar{P}_{D}\right]=\frac{5}{2}\left[\omega_{1}\right]+\frac{5}{2}\left[\omega_{2}\right]+\pi_{B}\left[\bar{P}_{D}\right]
$$

where $\pi_{B}$ is the orthogonal projection of $H^{2}\left(Y_{D} ; \mathbb{Q}\right)$ to $B$. Combining this with (1-8), we obtain

$$
\left[\bar{W}_{D}\right]=\frac{3}{2}\left[\omega_{1}\right]+\frac{9}{2}\left[\omega_{2}\right]+\pi_{B}\left[\bar{W}_{D}\right] .
$$

It follows from (1-8) that $\pi_{B}\left[\bar{P}_{D}\right]=\pi_{B}\left[\bar{W}_{D}\right]$. We derive these formulas in Section 11.

(3) In Section 12, we study the normal bundles of $\bar{W}_{D}$ and $\bar{P}_{D}$ in $Y_{D}$. The restriction of $\Omega Y_{D}$ to $\bar{W}_{D}^{\epsilon}$ is a line bundle $\Omega \bar{W}_{D}^{\epsilon}$. The degree of the normal bundle $N\left(\bar{W}_{D}^{\epsilon}\right)$ can be calculated in terms of $\Omega \bar{W}_{D}^{\epsilon}$. We obtain the following formula for the self intersection number of $\bar{W}_{D}^{\epsilon}$ :

$$
\left[\bar{W}_{D}^{\epsilon}\right]^{2}=\operatorname{deg} N\left(\bar{W}_{D}^{\epsilon}\right)=-\frac{2}{3} \operatorname{deg} \Omega \bar{W}_{D}^{\epsilon}=\frac{1}{3} \chi\left(W_{D}^{\epsilon}\right) .
$$

The proof of the middle equality involves defining a three-to-one map from a tubular neighborhood $\bar{W}_{D}^{\epsilon}$ to $\left(\Omega \bar{W}_{D}^{\epsilon}\right)^{-2}$ via an operation called "collapsing a 
saddle connection," which we introduce in Section 4.1. This operation replaces an Abelian differential together with a saddle connection joining a pair of simple zeros with an Abelian differential with a double zero.

Since $\bar{W}_{D}^{\epsilon}$ is transverse to the foliation $\mathcal{A}_{D}$ of $Y_{D}$, we also have the relation,

$$
N\left(\bar{W}_{D}^{\epsilon}\right) \cong T \mathcal{A}_{D}
$$

which together with (1-6) implies

$$
\left[\bar{W}_{D}^{\epsilon}\right]^{2}=\int_{W_{D}^{\epsilon}} \omega_{2}
$$

From this equation and (1-10), we obtain

$$
\int_{W_{D}^{\epsilon}} \omega_{2}=\frac{1}{3} \int_{W_{D}^{\epsilon}} \omega_{1} .
$$

(4) We show in Section 13 that the fundamental classes $\left[\bar{W}_{D}^{\epsilon}\right]$ are given by

$$
\left[\bar{W}_{D}^{\epsilon}\right]=\frac{3}{4}\left[\omega_{1}\right]+\frac{9}{4}\left[\omega_{2}\right]+\pi_{B}\left[W_{D}^{\epsilon}\right] \pm j,
$$

for some $j \in J$. The plan is to leave the coefficient of $\left[\omega_{1}\right]$ as an unknown $a$ and then to use our knowledge of $Y_{D}$ to write down equations involving the class $\left[\bar{W}_{D}^{\epsilon}\right]$ that allow us to solve for $a$. The following equations follow from Properties (b) and (c) of $Y_{D}$ :

$$
\begin{gathered}
{\left[\bar{W}_{D}^{0}\right] \cdot\left[\bar{P}_{D}\right]=0,} \\
{\left[\bar{W}_{D}^{0}\right] \cdot\left[\bar{W}_{D}^{0}\right]=\left[\bar{W}_{D}^{1}\right] \cdot\left[\bar{W}_{D}^{1}\right] .}
\end{gathered}
$$

These together with (1-11) give us enough equations to solve for $a$. Theorem 1.3 follows from (1-12) by pairing $-\left[\omega_{1}\right]$ with both sides as in the proof of Theorem 1.1.

Notes and references The orbits of the $\mathrm{SL}_{2} \mathbb{R}$ action on $\Omega_{1} \mathcal{M}_{g}$ project to immersions $\mathbb{H} \rightarrow \mathcal{M}_{g}$ of the hyperbolic plane into $\mathcal{M}_{g}$ which are isometric and totally geodesic with respect to the Teichmüller metric on $\mathcal{M}_{g}$. A totally geodesic immersion $\mathbb{H} \rightarrow \mathcal{M}_{g}$ is called a Teichmüller disk. It sometimes happens that a Teichmüller disk covers an algebraic curve $C$ in $\mathcal{M}_{g}$. In that case, the normalization of $C$ is called a Teichmüller curve. The curves $W_{D}$ are examples of Teichmüller curves in genus two. This action of $\mathrm{SL}_{2} \mathbb{R}$ is also closely related to the study of billiards in rational angled polygons, as well as the study of interval exchange maps. For information about Teichmüller curves, the action of $\mathrm{SL}_{2} \mathbb{R}$ on $\Omega \mathcal{M}_{g}$, and its relation with rational billiards and interval exchange maps, see for example Kerckhoff, Masur and Smillie [40], Masur [49], Masur and 
Tabachnikov [51], Veech [76; 77; 78; 79], Kontsevich [42], Zorich [84], Möller [60], or Bouw and Möller [16].

By analogy with the work of Ratner [67] on the dynamics of actions of groups generated by unipotent elements on homogeneous spaces, it is conjectured that the closure of every Teichmüller disk in $\mathcal{M}_{g}$ is an algebraic suborbifold of $\mathcal{M}_{g}$. McMullen's work establishes this conjecture in genus two for Teichmüller disks generated by Abelian differentials. McMullen analyzed the dynamics of $\mathrm{SL}_{2} \mathbb{R}$ on $\Omega_{1} \mathcal{M}_{2}$ in the series of papers $[52 ; 53 ; 54 ; 55 ; 56 ; 57]$. In these papers, he classified the closures of $\mathrm{SL}_{2} \mathbb{R}$ orbits in $\Omega_{1} \mathcal{M}_{2}$ and classified Teichmüller curves in genus two. He also introduced the curves $W_{D}$ in these papers and showed that they are all of the Teichmüller curves which are generated by an Abelian differential with a double zero. According to [52], the Teichmüller curve $W_{D}$ is primitive exactly when $D$ is nonsquare. The form generated by identifying opposite sides of a regular decagon has two simple zeros and generates a primitive Teichmüller curve $D_{10}$ in $\mathcal{M}_{2}$. In [56], McMullen showed that $D_{10}$ and the $W_{D}$ for $D$ nonsquare are in fact all of the primitive Teichmüller curves in $\mathcal{M}_{2}$ generated by an Abelian differential.

Teichmüller curves in genus two were also studied by Calta in [17], using the KenyonSmillie invariant introduced in [39].

Associated to every known $\mathrm{SL}_{2} \mathbb{R}$ orbit closure $S$ in $\Omega_{1} \mathcal{M}_{g}$ is a canonical finite, ergodic measure $\mu$. The volume of $\mu$ is an interesting quantity, which according to Veech [80] and Eskin and Masur [21] gives information about the dynamics of the geodesic flow of a generic Abelian differential $(X, \omega) \in S$ with respect to the canonical flat metric on $X$ determined by $\omega$. Volumes of orbit closures and their applications to dynamics are studied in Eskin and Okounkov [24], Eskin, Okounkov and Pandharipandhe [25], Eskin, Masur and Schmoll [22], Eskin, Masur and Zorich [23], Hubert and Lelièvre [35], Lelièvre and Royer [47], and Lelièvre [46].

A closed $\mathrm{SL}_{2} \mathbb{R}$-orbit lies over a Teichmüller curve $C$. In this case, the measure $\mu$ descends to a multiple of the hyperbolic area measure on $C$. These means that calculating the volume of $\mu$ is equivalent to calculating $\chi(C)$. In genus two, the Euler characteristics of the Teichmüller curves $W_{d^{2}}$ (as well as the volume of a related $\mathrm{SL}_{2} \mathbb{R}$-invariant measure on $X_{d^{2}}$ ) were studied in [22]. They established (1-2) by counting certain special Abelian differentials, called square-tiled surfaces. The connected components of $W_{D}$ were classified in [35] when $D$ is the square of a prime, and in [54] for arbitrary $D$. Theorem 1.4 calculating $\chi\left(W_{d^{2}}^{\epsilon}\right)$ was established in [35] when $d$ is prime and was conjectured for arbitrary $d$. In [47] Lelièvre and Royer established Theorem 1.4 independently by counting square-tiled surfaces using the theory of quasimodular forms. 
With the calculation of $\chi\left(W_{D}\right)$, we now know the Euler characteristics of all of the Teichmüller curves in $\mathcal{M}_{2}$ which are generated by Abelian differentials with a double zero. The number of cusps of $W_{D}$ was calculated by McMullen in [54]. We can also calculate the number of elliptic points of $W_{D}$ using known formulas for the numbers of elliptic points of $X_{D}$ and $P_{D}$. Thus we can calculate the genus of $W_{D}$ for any $D$. At this point we don't know the number of elliptic points on the components $W_{D}^{\epsilon}$, so we can't calculate the genus of these components, but we would conjecture that the two components have the same number of elliptic points and genera if $D$ is not square. The curve $W_{D}$ is defined over $\mathbb{Q}$, so the Galois automorphism $\sigma$ of $\mathbb{Q}(\sqrt{D})$ acts on the $\overline{\mathbb{Q}}$ points of $W_{D}$. An alternative approach to showing that $\chi\left(W_{D}^{0}\right)=\chi\left(W_{D}^{1}\right)$, as well as showing that the components have the same number of elliptic points and genera, would be to show that $\sigma$ permutes the two components of $W_{D}$.

Acknowledgments This paper is an expanded version of my Ph.D. thesis at Harvard. I would like to thank my advisor, Curt McMullen, for his invaluable help with every stage of this work. I would also like to thank Sabin Cautis, Alex Eskin, Florian Herzig, and Howard Masur for useful conversations, and I would like to thank Joe Harris and Yum-Tong Siu for reading an earlier version of this paper.

\section{Abelian surfaces and real multiplication}

We discuss in this section the necessary preliminaries involving Abelian surfaces. In Section 2.1, we discuss the Siegel modular varieties which parameterize Abelian varieties. In Section 2.2, we introduce real multiplication, and in Section 2.3, we discuss Hilbert modular surfaces.

\subsection{Abelian surfaces}

Abelian varieties A complex torus is a quotient $A=V / \Lambda$, where $\Lambda$ is a lattice in a finite dimensional complex vector space $V$. A principal polarization on $A$ is a Hermitian form $H$ on $V$ such that $\operatorname{Im} H$ takes integral values on $\Lambda \times \Lambda$, and the pairing

$$
\operatorname{Im} H: \Lambda \times \Lambda \rightarrow \mathbb{Z}
$$

is unimodular. A principally polarized Abelian variety is a complex torus equipped with a principal polarization.

Siegel modular varieties The Siegel upper half space is

$$
\mathcal{H}_{g}=\left\{Z \in M_{g}(\mathbb{C}): Z^{t}=Z, \operatorname{Im} Z>0\right\},
$$


an open subset in the $g(g+1) / 2$ dimensional space of symmetric matrices in $M_{g}(\mathbb{C})$. The group $\mathrm{Sp}_{2 g} \mathbb{R}$ acts on $\mathcal{H}_{g}$ as a group of biholomorphic transformations by

$$
\left(\begin{array}{ll}
\alpha & \beta \\
\gamma & \delta
\end{array}\right) \cdot Z=(\alpha Z+\beta)(\gamma Z+\delta)^{-1}
$$

Equip $\Lambda=\mathbb{Z}^{2 g}$ with the usual symplectic form defined by the matrix

$$
\left(\begin{array}{rr}
0 & I \\
-I & 0
\end{array}\right)
$$

Following [13], we define for each $Z \in \mathcal{H}_{g}$ a principally polarized Abelian surface $X_{Z}$ together with a symplectic isomorphism $\Lambda \rightarrow H_{1}\left(X_{Z} ; \mathbb{Z}\right)$. For $Z \in \mathcal{H}_{g}$, let

$$
\Lambda_{Z}=(Z, I) \Lambda,
$$

a lattice in $\mathbb{C}^{g}$. Let $X_{Z}=\mathbb{C}^{g} / \Lambda_{Z}$, and give $X_{Z}$ the polarization coming from the Hermitian form on $\mathbb{C}^{g}$ defined by the matrix

$$
(\operatorname{Im} Z)^{-1} \text {. }
$$

It can be shown that two given points $Z, Z^{\prime} \in \mathcal{H}_{g}$, the polarized Abelian surfaces $X_{Z}$ and $X_{Z^{\prime}}$ are isomorphic if and only if $Z$ and $Z^{\prime}$ are equivalent under the action of $\mathrm{Sp}_{2 g} \mathbb{Z}$.

The Siegel modular variety is $\mathcal{A}_{g}=\mathcal{H}_{g} / \mathrm{Sp}_{2 g} \mathbb{Z}$. From the previous paragraph, we obtain:

Theorem 2.1 The normal analytic space $\mathcal{A}_{g}$ is the moduli space of principally polarized Abelian varieties of dimension $g$.

Satake compactification Following [75], we briefly describe the Satake compactification $\widehat{\mathcal{A}}_{g}$ of $\mathcal{A}_{g}$, introduced by Satake in [69].

The transformation,

$$
Z \mapsto(1+i Z)(1-i Z)^{-1},
$$

maps $\mathcal{H}_{g}$ biholomorphically onto the bounded symmetric domain

$$
\mathcal{D}_{g}=\left\{Z \in M_{g}(\mathbb{C}): Z=Z^{t}, 1_{g}-Z \bar{Z}>0\right\} .
$$

For $r \leq g$, we map $\mathcal{D}_{r}$ into $\overline{\mathcal{D}}_{g}$ by

$$
Z \mapsto\left(\begin{array}{cc}
Z & 0 \\
0 & 1 g-r
\end{array}\right)
$$


and let

$$
\mathcal{D}_{g}^{*}=\bigcup_{0 \leq r \leq g} \bigcup_{h \in \operatorname{Sp}_{2 g} \mathbb{Q}} h \mathcal{D}_{r}
$$

Satake defined a natural topology on $\mathcal{D}_{g}^{*}$ for which the action of $\mathrm{Sp}_{2 g} \mathbb{Q}$ extends continuously to an action on $\mathcal{D}_{g}^{*}$. With this topology, the Satake showed that the quotient $\widehat{\mathcal{A}}_{g}=\mathcal{D}_{g}^{*} / \mathrm{Sp}_{2 g} \mathbb{Z}$ is a compact, normal complex analytic space which contains one copy of $\mathcal{A}_{r}$ for each $r \leq g$. Baily showed in [4] that $\widehat{\mathcal{A}}_{g}$ has the structure of a normal projective variety.

\subsection{Real multiplication}

Quadratic orders Let $K$ be a quadratic field or $\mathbb{Q} \oplus \mathbb{Q}$. A quadratic order is a subring $\mathcal{O}$ of $K$ such that $1 \in \mathcal{O}$ and $\mathcal{O} \otimes \mathbb{Q}=K$. Any quadratic order is isomorphic to one of

$$
\mathcal{O}_{D}=\mathbb{Z}[T] /\left(T^{2}+b T+c\right),
$$

where $b, c \in \mathbb{Z}$ and $b^{2}-4 c=D$. The isomorphism class of $\mathcal{O}_{D}$ only depends on $D$, so this defines a unique quadratic order for every nonzero integer $D \equiv 0$ or $1(\bmod 4)$. This integer $D$ is called the discriminant of $\mathcal{O}_{D}$, and $D$ is a fundamental discriminant if $D$ is not of the form $f^{2} E$ for some integers $f$ and $E$ with $E \equiv 0$ or $1(\bmod 4)$ and $f>1$.

If $D$ is not square, then $\mathcal{O}_{D}$ is a subring of $\mathbb{Q}(\sqrt{D})$, and $\mathcal{O}_{D}$ is the ring of integers of $\mathbb{Q}(\sqrt{D})$ if and only if $D$ is a fundamental discriminant. Otherwise $\mathcal{O}_{D}$ is a subring of the ring of integers. If $D$ is square, then $\mathcal{O}_{D}$ is a subring of $\mathbb{Q} \oplus \mathbb{Q}$. In that case,

$$
\mathcal{O}_{d^{2}}=\{(x, y) \in \mathbb{Z} \times \mathbb{Z}: x \equiv y \quad(\bmod d)\} .
$$

We will regard $\mathbb{Q} \oplus \mathbb{Q}$ as an extension of $\mathbb{Q}$ by the diagonal map $\mathbb{Q} \rightarrow \mathbb{Q} \oplus \mathbb{Q}$. The Galois automorphism of $\mathbb{Q} \oplus \mathbb{Q}$ is $(x, y)^{\prime}=(y, x)$, and we can use this to define norm and trace on $\mathbb{Q} \oplus \mathbb{Q}$ as for a field.

For the rest of this paper, fix two nonzero homomorphisms $\iota_{i}: \mathcal{O}_{D} \rightarrow \mathbb{R}$. If $D$ is not square, then $\iota_{i}$ is an embedding, and if $D$ is square, then $\iota_{i}$ is induced by one of the two projections $\mathbb{Z} \oplus \mathbb{Z} \rightarrow \mathbb{Z}$. We will often abuse terminology and call $\iota_{i}$ an embedding even when $D$ is square. We will also use the notation $\lambda^{(i)}=\iota_{i}(\lambda)$.

Inverse different Given a quadratic order $\mathcal{O}_{D}$, the inverse different is the fractional ideal

$$
\mathcal{O}_{D}^{\vee}=\left\{x \in K_{D}: \operatorname{Tr}(x y) \in \mathbb{Z}, \text { for all } y \in \mathcal{O}_{D}\right\} .
$$

More concretely,

$$
\mathcal{O}_{D}^{\vee}=\frac{1}{\sqrt{D}} \mathcal{O}_{D}
$$


where if $D=d^{2}$, we interpret $\sqrt{D}$ to be $(d,-d)$.

We equip $\mathcal{O}_{D} \oplus \mathcal{O}_{D}^{\vee}$ with the unimodular symplectic pairing

$$
\left\langle\left(x_{1}, y_{1}\right),\left(x_{2}, y_{2}\right)\right\rangle=\operatorname{Tr}\left(x_{1} y_{2}-x_{2} y_{1}\right) \text {. }
$$

Real multiplication Consider an Abelian surface $A=V / \Lambda$. Real multiplication by $\mathcal{O}_{D}$ on $A$ is a monomorphism $\rho: \mathcal{O}_{D} \rightarrow \operatorname{End}(A)$ (where $\operatorname{End}(A)$ is the ring of holomorphic endomorphisms of $A$ ) with the following properties:

- For each $\lambda \in \mathcal{O}_{D}$, the lift $\tilde{\rho}(\lambda): V \rightarrow V$ is self-adjoint with respect to the Hermitian form on $V$ given by the polarization of $A$.

- $\rho$ is proper in the sense that it doesn't extend to a monomorphism

$$
\rho^{\prime}: \mathcal{O}_{E} \rightarrow \operatorname{End}(A)
$$

for some $\mathcal{O}_{E} \supset \mathcal{O}_{D}$ - that is, for some $\mathcal{O}_{E}$ with $E=f^{2} D$ for some $f>1$.

Let $\Omega(A) \cong V^{*}$ be the space of holomorphic one-forms on $A$. Since the real multiplication is self-adjoint, there is an eigenspace decomposition

$$
\Omega(A)=\Omega^{1}(A) \oplus \Omega^{2}(A) .
$$

We order the eigenspaces so that $\rho(\lambda) \cdot \omega=\lambda^{(i)} \omega$ for $\omega \in \Omega^{i}(A)$. We call an eigenform in $\Omega^{i}(A)$ an $i$-eigenform.

\subsection{Hilbert modular surfaces}

For any quadratic discriminant $D$, let $K_{D}=\mathcal{O}_{D} \otimes \mathbb{Q}$, which is $\mathbb{Q}(\sqrt{D})$ if $D$ is not square and is $\mathbb{Q} \oplus \mathbb{Q}$ if $D$ is square. Define the group

$$
\operatorname{SL}\left(\mathcal{O}_{D} \oplus \mathcal{O}_{D}^{\vee}\right)=\left\{\left(\begin{array}{ll}
a & b \\
c & d
\end{array}\right) \in \mathrm{SL}_{2} K_{D}: a \in \mathcal{O}_{D}, b \in\left(\mathcal{O}_{D}^{\vee}\right)^{-1}, c \in \mathcal{O}_{D}^{\vee}, d \in \mathcal{O}_{D}\right\}
$$

$\mathrm{SL}\left(\mathcal{O}_{D} \oplus \mathcal{O}_{D}^{\vee}\right)$ has two embeddings in $\mathrm{SL}_{2} \mathbb{R}$ induced by the two embeddings $\iota_{i}: K_{D} \rightarrow$ $\mathbb{R}$.

Definition The Hilbert modular surface of discriminant $D$ is the quotient,

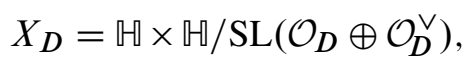

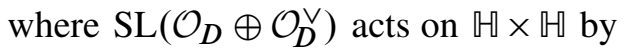

$$
\left(\begin{array}{ll}
a & b \\
c & d
\end{array}\right) \cdot\left(z_{1}, z_{2}\right)=\left(\frac{a^{(1)} z_{1}+b^{(1)}}{c^{(1)} z_{1}+d^{(1)}}, \frac{a^{(2)} z_{2}+b^{(2)}}{c^{(2)} z_{2}+d^{(2)}}\right) .
$$


There is an isomorphism $\mathrm{SL}\left(\mathcal{O}_{D} \oplus \mathcal{O}_{D}^{\vee}\right) \rightarrow \mathrm{SL}_{2} \mathcal{O}_{D}$ defined by

$$
\left(\begin{array}{ll}
a & b \\
c & d
\end{array}\right) \mapsto\left(\begin{array}{cc}
a & \frac{1}{\sqrt{D}} b \\
\sqrt{D} c & d
\end{array}\right),
$$

and the map $T: \mathbb{M} \times \mathbb{W} \rightarrow \mathbb{W} \times(-\mathbb{W})$ which is the product of the identity on the first factor and complex conjugation on the second factor induces an isomorphism

$$
X_{D} \cong \mathbb{H} \times(-\mathbb{H}) / \mathrm{SL}_{2} \mathcal{O}_{D}
$$

Theorem 2.2 The Hilbert modular surface $X_{D}$ is the moduli space of all pairs $(A, \rho)$, where $A$ is a principally polarized Abelian surface, and $\rho: \mathcal{O}_{D} \rightarrow \operatorname{End}(A)$ is a choice of real multiplication on $A$.

Sketch of proof (following Mcmullen [58]) Given $\tau=\left(\tau_{1}, \tau_{2}\right) \in \mathbb{U} \times \mathbb{M}$, let

$$
\phi_{\tau}: \mathcal{O}_{D} \oplus \mathcal{O}_{D}^{\vee} \rightarrow \mathbb{C}^{2}
$$

be the embedding

$$
\phi_{\tau}(x, y)=\left(x^{(1)}+y^{(1)} \tau_{1}, x^{(2)}+y^{(2)} \tau_{2}\right) .
$$

Let $A_{\tau}=\mathbb{C}^{2} / \phi_{\tau}\left(\mathcal{O}_{D} \oplus \mathcal{O}_{D}^{\vee}\right)$ with the principal polarization induced by the symplectic pairing on $\mathcal{O}_{D} \oplus \mathcal{O}_{D}^{\vee}$. This polarization is also given by the Hermitian form

$$
H_{\tau}(z, w)=\frac{1}{\operatorname{Im} \tau_{1}} z_{1} \bar{w}_{1}+\frac{1}{\operatorname{Im} \tau_{2}} z_{2} \bar{w}_{2} .
$$

Define real multiplication on $A_{\tau}$ by

$$
\lambda \cdot\left(z_{1}, z_{2}\right)=\left(\lambda^{(1)} z_{1}, \lambda^{(2)} z_{2}\right) .
$$

We thus get a map from $\mathbb{H} \times \mathbb{U}$ to the set of all triples $(A, \rho, \phi)$, where $(A, \rho)$ is a principally polarized Abelian surface with real multiplication by $\mathcal{O}_{D}$, and $\phi$ is a choice of an $\mathcal{O}_{D}$-linear, symplectic isomorphism $\phi: \mathcal{O}_{D} \oplus \mathcal{O}_{D}^{\vee} \rightarrow H_{1}(A ; \mathbb{Z})$.

Given

$$
g=\left(\begin{array}{ll}
a & b \\
c & d
\end{array}\right) \in \mathrm{SL}_{2} K_{D}
$$

let

$$
g^{*}=\left(\begin{array}{cc}
a & -b \\
-c & d
\end{array}\right),
$$

and define

$$
\chi(g, \tau)=\left(\begin{array}{cc}
\left(c^{(1)} \tau_{1}+d^{(1)}\right)^{-1} & 0 \\
0 & \left(c^{(2)} \tau_{2}+d^{(2)}\right)^{-1}
\end{array}\right)
$$


We have the following commutative diagram.

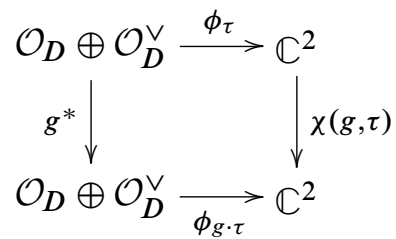

Thus $\chi(g, \tau)$ induces an isomorphism between $A_{\tau}$ and $A_{g \cdot \tau}$ which preserves the polarizations and commutes with the action of real multiplication. We then get a map from $X_{D}$ to the set of all principally polarized Abelian surfaces with a choice of real multiplication, which can be shown to be a bijection.

Replacing each pair $(A, \rho)$ with $\left(A, \rho^{\prime}\right)$, where $\rho^{\prime}$ is the composition of $\rho$ with the Galois automorphism of $\mathcal{O}_{D}$, induces an involution $\tau$ of $X_{D}$. The lift of $\tau$ to the universal cover $\mathbb{H} \times \mathbb{U}$ of $X_{D}$ is given by $\tilde{\tau}\left(z_{1}, z_{2}\right)=\left(z_{2}, z_{1}\right)$.

There is a natural map $j: X_{D} \rightarrow \mathcal{A}_{2}$ which forgets the choice of real multiplication. This map $j$ is generically two to one and is equivariant with respect to $\tau$.

Baily-Borel compactification We can regard the boundary $\partial \llbracket \subset \mathbb{P}^{1}(\mathbb{C})$ as $\mathbb{P}^{1}(\mathbb{R}) \cong$ $\mathbb{R} \cup\{\infty\}$. Given a real quadratic field $K \subset \mathbb{R}$, define an embedding $\mathbb{P}^{1}(K) \rightarrow \mathbb{P}(\mathbb{R})^{2}$ by

$$
[x: y] \mapsto\left([x: y],\left[x^{\prime}: y^{\prime}\right]\right) .
$$

When $D$ is not square, define via this embedding

$$
(\mathbb{H} \times \mathbb{M})_{D}=(\mathbb{H} \times \mathbb{M}) \cup \mathbb{P}^{1}\left(K_{D}\right) .
$$

When $D$ is square, define

$$
(\mathbb{W} \times \mathbb{U})_{D}=\left(\mathbb{H} \cup \mathbb{P}^{1}(\mathbb{Q})\right) \times\left(\mathbb{U} \cup \mathbb{P}^{1}(\mathbb{Q})\right) .
$$

We give $\mathbb{U} \cup \mathbb{P}^{1}(\mathbb{Q})$ the usual topology where if $r \in \mathbb{P}^{1}(\mathbb{Q})$, then a basis of open neighborhoods of $r$ is given by sets of the form $U \cup\{r\}$, where $U \subset \mathbb{M}$ is an open horoball resting on $r$. We then give $(\mathbb{H} \times \mathbb{U})_{d^{2}}$ the product topology. When $D$ is not square, there is a similar natural topology on $(\mathbb{H} \times \mathbb{U})_{D}$, which is described in [75].

The action of $\operatorname{SL}\left(\mathcal{O}_{D} \oplus \mathcal{O}_{D}^{\vee}\right)$ on $\mathbb{M} \times \mathbb{~ e x t e n d s ~ c o n t i n u o u s l y ~ t o ~}(\mathbb{M} \times \mathbb{M})_{D}$. The quotient,

$$
\widehat{X}_{D}=(\mathbb{H} \times \mathbb{U})_{D} / \operatorname{SL}\left(\mathcal{O}_{D} \oplus \mathcal{O}_{D}^{\vee}\right),
$$

is compact and Hausdorff. The space $\hat{X}_{D}$ is the Baily-Borel compactification of $X_{D}$. 
Theorem 2.3 (Baily [4]) The compactification $\widehat{X}_{D}$ is a normal, projective, algebraic variety.

When $D$ is not square, $\hat{X}_{D} \backslash X_{D}$ consists of finitely many points, which we will call the cusps of $X_{D}$. When $D=d^{2}$, the image of $\mathbb{P}^{1}(\mathbb{Q}) \times \mathbb{P}^{1}(\mathbb{Q})$ in $\hat{X}_{d^{2}}$ also consists of finitely many points which we will call the cusps of $\hat{X}_{d^{2}}$. In $\hat{X}_{d^{2}}$, let

$$
\begin{aligned}
& R_{d^{2}}^{1}=\pi\left(\bigcup_{r \in \mathbb{P}^{1}(\mathbb{Q})} \mathbb{H} \times\{r\}\right), \\
& R_{d^{2}}^{2}=\pi\left(\bigcup_{r \in \mathbb{P}^{1}(\mathbb{Q})}\{r\} \times \mathbb{U}\right),
\end{aligned}
$$

and let

where $\pi:(\mathbb{H} \times \mathbb{U})_{D} \rightarrow \widehat{X}_{D}$ is the natural quotient map. Then we have a disjoint union

$$
\widehat{X}_{d^{2}}=X_{d^{2}} \amalg R_{d^{2}}^{1} \amalg R_{d^{2}}^{2} \amalg C,
$$

where $C$ is the set of cusps of $\hat{X}_{d^{2}}$.

For $d \in \mathbb{N}$, define

$$
\Gamma_{1}(d)=\left\{\left(\begin{array}{ll}
a & b \\
c & e
\end{array}\right) \in \mathrm{SL}_{2} \mathbb{Z}: a \equiv e \equiv 1 \quad(\bmod d), \text { and } c \equiv 0 \quad(\bmod d)\right\} .
$$

Proposition 2.4 The curve $R_{d^{2}}^{i}$ is irreducible, and

$$
R_{d^{2}}^{i} \cong \mathbb{H} / \Gamma_{1}(d)
$$

Proof By (2-2), we can regard $\mathrm{SL}_{2} \mathcal{O}_{d^{2}}$ as

$$
\left\{(A, B) \in\left(\mathrm{SL}_{2} \mathbb{Z}\right)^{2}: A \equiv B \quad(\bmod d)\right\} .
$$

Since $\mathrm{SL}_{2} \mathbb{Z}$ acts transitively on $\mathbb{P}^{1}(\mathbb{Q})$, we have

$$
R_{d^{2}}^{1}=\mathbb{} \times\{\infty\} / \operatorname{Stab}_{\sharp \times\{\infty\}} .
$$

The matrices $A$ in the pair $(A, B) \in \operatorname{Stab}_{\sharp \times\{\infty\}}$ are exactly the matrices which are congruent to an upper triangular matrix $\bmod d$. Thus $R_{d^{2}}^{1}$ is as claimed.

When $D$ is not square, the cusps of $\widehat{X}_{D}$ are complicated singularities; however, when $D$ is square, they are just orbifold singularities. 
Proposition 2.5 The Baily-Borel compactification $\widehat{X}_{d^{2}}$ is a compact, complex orbifold with singularities at the elliptic points of $X_{d^{2}}$, the elliptic points of $R_{d^{2}}^{i}$, and possibly the cusps of $\hat{X}_{d^{2}}$.

Proof Consider the principal congruence subgroup

$$
\Gamma(d)=\left\{A \in \mathrm{SL}_{2} \mathbb{Z}: A \equiv I \quad(\bmod d)\right\} .
$$

The product,

$$
\Gamma(d) \times \Gamma(d) \subset \mathrm{SL}_{2} \mathcal{O}_{d^{2}},
$$

is a finite index, normal subgroup. We have

$$
(\mathbb{H} \times \mathbb{U})_{d^{2}} /(\Gamma(d) \times \Gamma(d))=\overline{\mathbb{U} / \Gamma(d)} \times \overline{\mathbb{H} / \Gamma(d)},
$$

which is a manifold, so

$$
\widehat{X}_{d^{2}}=(\overline{\mathbb{W} / \Gamma(d)} \times \overline{\mathbb{H} / \Gamma(d)}) / G
$$

where $G=\mathrm{SL}_{2} \mathcal{O}_{d^{2}} /(\Gamma(d) \times \Gamma(d))$, a finite group. Thus $\hat{X}_{d^{2}}$ is compact orbifold with singularities at the fixed points of $G$.

The involution $\left(z_{1}, z_{2}\right) \mapsto\left(z_{2}, z_{1}\right)$ of $\mathbb{M} \times \mathbb{W}$ extends continuously to $(\mathbb{M} \times \mathbb{H})_{D}$, so the involution $\tau$ extends to an involution $\tau$ of $\hat{X}_{D}$, which preserves the cusps of $\hat{X}_{D}$. If $D=d^{2}$, then we also have $\tau\left(R_{d^{2}}^{1}\right)=R_{d^{2}}^{2}$.

Theorem 2.6 The natural map $j: X_{D} \rightarrow \mathcal{A}_{2}$ extends to a finite morphism $j: \hat{X}_{D} \rightarrow$ $\widehat{\mathcal{A}}_{2}$ which sends the cusps of $\hat{X}_{D}$ to the point $\mathcal{A}_{0} \in \mathcal{A}_{2}$ and sends the curves $R_{D}^{i}$ to $\mathcal{A}_{1}$.

This allows us to define $\hat{X}_{D}$ alternatively in terms of the image of $X_{D}$ in $\widehat{\mathcal{A}}_{2}$. Recall the notion of the normalization of a variety in a finite algebraic extension of its function field discussed in Appendix A.

Corollary 2.7 The Baily-Borel compactification $\hat{X}_{D}$ is the normalization of the closure of $j\left(X_{D}\right)$ in $\widehat{\mathcal{A}}_{2}$ in the field $K\left(X_{D}\right)$, the function field of $X_{D}$.

Line bundles on $X_{\boldsymbol{D}}$ We now discuss some bundles on $X_{D}$, which will be used in Section 9. For $i=1$ or 2 , define an action of $\operatorname{SL}\left(\mathcal{O}_{D} \oplus \mathcal{O}_{D}^{\vee}\right)$ on $(\mathbb{M} \times \mathbb{U}) \times \mathbb{C}$ by

$$
\left(\begin{array}{ll}
a & b \\
c & d
\end{array}\right) \cdot\left(z_{1}, z_{2}, w\right)=\left(\frac{a^{(1)} z_{1}+b^{(1)}}{c^{(1)} z_{1}+d^{(1)}}, \frac{a^{(2)} z_{2}+b^{(2)}}{c^{(2)} z_{2}+d^{(2)}},\left(c^{(i)} z_{i}+d^{(i)}\right)^{2} w\right) .
$$

The quotient is a line bundle over $X_{D}$, which we call $L_{i}$. 
We define a Hermitian metric $\tilde{h}_{i}$ on $(\mathbb{H} \times \mathbb{H}) \times \mathbb{C}$ by defining on the fiber over $\left(z_{1}, z_{2}\right)$,

$$
\tilde{h}_{i}(w, w)=y_{i}^{2}|w|^{2}
$$

The metric $\tilde{h}_{i}$ is $\operatorname{SL}\left(\mathcal{O}_{D} \oplus \mathcal{O}_{D}^{\vee}\right)$-invariant, so it descends to a Hermitian metric $h_{i}$ on $L_{i}$. The Chern form of $\tilde{h}_{i}$ is,

$$
\begin{aligned}
c_{1}\left(L_{i}, h_{i}\right) & =-\frac{i}{\pi} \partial \bar{\partial} \log \left(y_{i}\right) \\
& =\frac{1}{2 \pi} \frac{d x_{i} \wedge d y_{i}}{y_{i}^{2}},
\end{aligned}
$$

so the Chern form of $h_{i}$ is $c_{1}\left(L_{i}, h_{i}\right)=\omega_{i}$, where $\omega_{i}$ is the 2 -form on $X_{D}$ covered by the form (2-6) on $\mathbb{U} \times \mathbb{U}$.

Define line bundles

$$
\Omega^{i} X_{D}=\left\{(A, \omega): A \in X_{D} \text { and } \omega \in \Omega^{i}(A)\right\}
$$

and

$$
Q^{i} X_{D}=\left(\Omega^{i} X_{D}\right)^{\otimes 2},
$$

for $i=1$ or 2 . We will mostly use these bundles when $i=1$, so we will write $\Omega X_{D}$ or $Q X_{D}$ for $\Omega^{1} X_{D}$ or $Q^{1} X_{D}$.

For $A \in X_{D}$, the polarization defines a Hermitian metric on $\Omega(A)$, which restricts to a Hermitian metric on $\Omega^{i}(A)$. Put this Hermitian metric on each $\Omega^{i}(A)$ to define a Hermitian metric $h_{\Omega}^{i}$ on $\Omega^{i} X_{D}$. Let $h_{Q}^{i}$ be the induced metric on $Q^{i} X_{D}$.

Proposition 2.8 There is an isomorphism $L_{i} \rightarrow Q^{i} X_{D}$ which preserves the Hermitian metrics on these bundles. Thus,

$$
c_{1}\left(Q^{i} X_{D}, h_{Q}^{i}\right)=\omega_{i}
$$

Proof Define a map $f:(\mathbb{W} \times \mathbb{U}) \times \mathbb{C} \rightarrow Q^{i} X_{D}$ by

$$
(\tau, w) \mapsto\left(A_{\tau}, w d z_{i}^{2}\right),
$$

where $A_{\tau}=\mathbb{C}^{2} / \phi_{\tau}\left(\mathcal{O}_{D} \oplus \mathcal{O}_{D}^{\vee}\right)$ is as in the proof of Theorem 2.2. With $\chi(g, \tau)$ as in (2-4), we have

$$
\chi(g, \tau)_{*} d z_{i}^{2}=\left(c^{(i)} \tau_{i}+d^{(i)}\right)^{2} d z_{i}^{2} .
$$

This means that $f$ descends to an isomorphism $L_{i} \rightarrow Q^{i} X_{D}$ as claimed. The fact that this isomorphism preserves the Hermitian metrics follows directly from the formula (2-3) for the polarization. 


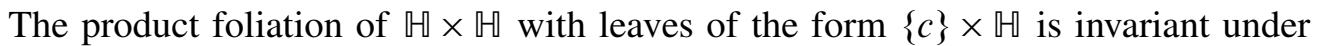
$\operatorname{SL}\left(\mathcal{O}_{D} \oplus \mathcal{O}_{D}^{\vee}\right)$. Let $\mathcal{A}_{D}$ be the induced foliation of $X_{D}$, and let

$$
T^{*} \mathcal{A}_{D} \rightarrow X_{D}
$$

be the line bundle whose fiber over a point $p$ is the cotangent bundle to the leaf of $\mathcal{A}_{D}$ through $p$. We give the leaves of $\mathcal{A}_{D}$ their hyperbolic metric, and give $T^{*} \mathcal{A}_{D}$ the induced Hermitian metric $h_{\mathcal{A}_{D}}$. The following is easy to check.

Proposition 2.9 There is an isomorphism $L_{2} \rightarrow T^{*} \mathcal{A}_{D}$ preserving the Hermitian metrics. Thus,

$$
c_{1}\left(T^{*} \mathcal{A}_{D}, h_{\mathcal{A}_{D}}\right)=\omega_{2}
$$

The following relation between $\Omega X_{D}$ and $T^{*} \mathcal{A}_{D}$ will be used in Section 10 .

Proposition 2.10 For any $p \in X_{D}$, there is a neighborhood $U$ of $p$ in the leaf $L$ of $\mathcal{A}_{D}$ through $p$ and a section $\omega$ of $\Omega X_{D}$ over $U$ such that the periods of the forms $\omega(z)$ are constant over $U$.

Proof Let

$$
\left(\tau_{1}, \tau_{2}\right) \in \tilde{X}_{D}=\mathbb{H} \times \mathbb{H}
$$

lie over $p$ in the universal cover of $X_{D}$. Define a section $s$ of $\Omega \tilde{X}_{D}$ by

$$
s\left(\tau_{1}, \tau_{2}\right)=d z_{1} \in \Omega^{1}\left(A_{\left(\tau_{1}, \tau_{2}\right)}\right),
$$

with $A_{\tau}$ as in the proof of Theorem 2.2. By the definition of $A_{\tau}$, the periods of $s\left(\tau_{1}, \tau_{2}\right)$ only depend on $\tau_{1}$, so are constant along $\mathcal{A}_{D}$.

Euler characteristic of $\boldsymbol{X}_{\boldsymbol{D}}$ We now discuss the Euler characteristic $\chi\left(X_{D}\right)$, which by the generalized Gauss-Bonnet theorem is given by the volume,

$$
\chi\left(X_{D}\right)=\int_{X_{D}} \omega_{1} \wedge \omega_{2}
$$

where $\omega_{i}$ are the 2 -forms on $X_{D}$ defined by (2-6). The volume of $X_{D}$ was calculated by Siegel when $D$ is a fundamental discriminant.

For any number field $K$, the Dedekind zeta-function of $K$ is defined by

$$
\zeta_{K}(s)=\sum_{\mathfrak{a}} \frac{1}{N_{\mathbb{Q}}^{K}(\mathfrak{a})^{s}},
$$


where the sum is over all nonzero ideals $\mathfrak{a}$ in ring of integers in $K$. If $K=\mathbb{Q}$, then $\zeta_{K}$ is just the Riemann zeta-function. The definition of $\zeta_{K}$ also makes sense if $K=\mathbb{Q} \oplus \mathbb{Q}$. In that case,

$$
\zeta_{\mathbb{Q} \oplus \mathbb{Q}}(s)=\zeta_{\mathbb{Q}}(s)^{2} .
$$

$\zeta_{K}$ can be analytically continued to a meromorphic function on $\mathbb{C}$ which has a simple pole at $s=1$.

Theorem 2.11 (Siegel [73]) When $D$ is a fundamental discriminant, and $D \neq 1$,

$$
\chi\left(X_{D}\right)=2 \zeta_{K_{D}}(-1)
$$

We will also want to know $\chi\left(X_{D}\right)$ when $D$ is not a fundamental discriminant. For integers $a$ and $b$ with $b>0$, let

$$
\left(\frac{a}{b}\right)
$$

be the Kronecker symbol, defined in Miyake [59, p 82].

Theorem 2.12 If $D$ is a fundamental discriminant, and $f \in \mathbb{N}$, then

$$
\chi\left(X_{f^{2} D}\right)= \begin{cases}1 / 36 & \text { if } D=f=1 ; \\ 1 / 6 & \text { if } D=1 \text { and } f=2 .\end{cases}
$$

Otherwise,

$$
\chi\left(X_{f^{2} D}\right)=2 f^{3} \zeta_{K_{D}}(-1) \sum_{r \mid f}\left(\frac{D}{r}\right) \frac{\mu(r)}{r^{2}}
$$

Remark When $D=1,(2-7)$ reduces to

$$
\chi\left(X_{d^{2}}\right)=\frac{1}{72} d^{3} \sum_{r \mid d} \frac{\mu(r)}{r^{2}} .
$$

We will derive Theorem 2.12 from Theorem 2.11 in a sequence of lemmas.

Lemma 2.13 We have

$$
\left[\mathrm{PSL}_{2} \mathbb{Z} \times \mathrm{PSL}_{2} \mathbb{Z}: \mathrm{PSL}_{2} \mathrm{O}_{4}\right]=6
$$

and $\quad\left[\mathrm{PSL}_{2} \mathbb{Z} \times \mathrm{PSL}_{2} \mathbb{Z}: \mathrm{PSL}_{2} \mathcal{O}_{d^{2}}\right]=\frac{1}{2} d^{3} \sum_{r \mid d} \frac{\mu(r)}{r^{2}} \quad$ if $d>2$. 
Proof We will regard $\mathrm{SL}_{2} \mathcal{O}_{d^{2}}$ as the subgroup of $\mathrm{SL}_{2} \mathbb{Z} \times \mathrm{SL}_{2} \mathbb{Z}$ given in (2-5). The natural map,

$$
\mathrm{SL}_{2} \mathbb{Z} \rightarrow \mathrm{SL}_{2}(\mathbb{Z} / d)
$$

is surjective by [59, Theorem 4.2.1] and has kernel the principal congruence subgroup $\Gamma(d)$, so we have the exact sequence

$$
0 \rightarrow \Gamma(d) \times \Gamma(d) \rightarrow \mathrm{SL}_{2} \mathcal{O}_{d^{2}} \rightarrow \mathrm{SL}_{2}(\mathbb{Z} / d) \rightarrow 0 .
$$

Thus,

$$
\begin{aligned}
{\left[\mathrm{SL}_{2} \mathcal{O}_{d^{2}}: \Gamma(d) \times \Gamma(d)\right] } & =\left|\mathrm{SL}_{2}(\mathbb{Z} / d)\right| \\
{\left[\mathrm{SL}_{2} \mathbb{Z} \times \mathrm{SL}_{2} \mathbb{Z}: \mathrm{SL}_{2} \mathcal{O}_{d^{2}}\right] } & =\frac{\left[\mathrm{SL}_{2} \mathbb{Z} \times \mathrm{SL}_{2} \mathbb{Z}: \Gamma(d) \times \Gamma(d)\right]}{\left[\mathrm{SL}_{2} \mathcal{O}_{d^{2}}: \Gamma(d) \times \Gamma(d)\right]} \\
& =\left|\mathrm{SL}_{2}(\mathbb{Z} / d)\right| .
\end{aligned}
$$

By [59, Theorem 4.2.4],

$$
\left|\mathrm{SL}_{2}(\mathbb{Z} / d)\right|=d^{3} \sum_{r \mid d} \frac{\mu(r)}{r^{2}} .
$$

The kernel of $\mathrm{SL}_{2} \mathbb{Z} \times \mathrm{SL}_{2} \mathbb{Z} \rightarrow \mathrm{PSL}_{2} \mathbb{Z} \times \mathrm{PSL}_{2} \mathbb{Z}$ has order 4. The kernel of $\mathrm{SL}_{2} \mathrm{O}_{d^{2}} \rightarrow$ $\mathrm{PSL}_{2} \mathcal{O}_{d^{2}}$ has order 4 if $d=2$ and order 2 if $d>2$. The desired formulas follow from this and (2-8).

Lemma 2.14 For any fundamental discriminant $D \neq 1$, we have

$$
\begin{aligned}
{\left[\mathrm{PSL}_{2} \mathcal{O}_{D}: \mathrm{PSL}_{2} \mathcal{O}_{f^{2} D}\right] } & =f^{3} \prod_{p \mid f}\left(1-\left(\frac{D}{p}\right) p^{-2}\right) \\
& =f^{3} \sum_{r \mid f}\left(\frac{D}{r}\right) \frac{\mu(r)}{r^{2}} .
\end{aligned}
$$

Proof Since the map $\mathrm{SL}_{2} \mathrm{O}_{f^{2} D} \rightarrow \mathrm{PSL}_{2} \mathcal{O}_{f^{2} D}$ has kernel $\pm I$, we have

$$
\left[\mathrm{PSL}_{2} \mathcal{O}_{D}: \mathrm{PSL}_{2} \mathcal{O}_{f^{2} D}\right]=\left[\mathrm{SL}_{2} \mathcal{O}_{D}: \mathrm{SL}_{2} \mathcal{O}_{f^{2} D}\right]
$$

The natural map,

$$
\mathrm{SL}_{2} \mathcal{O}_{D} / \mathrm{SL}_{2} \mathcal{O}_{f^{2} D} \rightarrow \prod_{\substack{p \mid f \\ p \text { prime }}} \mathrm{SL}_{2}\left(\mathcal{O}_{D} / p\right) / \mathrm{SL}_{2}(\mathbb{Z} / p)
$$

is a bijection. Thus, we need only to show that

$$
\left[\mathrm{SL}_{2}\left(\mathcal{O}_{D} / p\right): \mathrm{SL}_{2}(\mathbb{Z} / p)\right]=p^{3}-\left(\frac{D}{p}\right) p
$$


for every prime $p \mid f$.

First, suppose that $(D / p)=-1$. This means that $p$ remains prime in $\mathcal{O}_{D}$, so $\mathcal{O}_{D} / p \cong$ $\mathbb{F}_{p^{2}}$, where we write $\mathbb{F}_{q}$ for the unique finite field of order $q$. It is easy to show that

$$
\left|\mathrm{SL}_{2} \mathbb{F}_{q}\right|=q^{3}-q
$$

for any $q$. Thus

$$
\left[\mathrm{SL}_{2} \mathbb{F}_{p^{2}}: \mathrm{SL}_{2} \mathbb{F}_{p}\right]=p^{3}+p
$$

as desired.

Now suppose that $(D / p)=1$. This means that $p$ splits in $\mathcal{O}_{D}$, so $\mathcal{O}_{D} / p \cong \mathbb{F}_{p} \oplus \mathbb{F}_{p}$. Therefore,

$$
\begin{aligned}
{\left[\mathrm{SL}_{2}\left(\mathcal{O}_{D} / p\right): \mathrm{SL}_{2}(\mathbb{Z} / p)\right] } & =\left[\mathrm{SL}_{2} \mathbb{F}_{p} \times \mathrm{SL}_{2} \mathbb{F}_{p}: \mathrm{SL}_{2} \mathbb{F}_{p}\right] \\
& =\left|\mathrm{SL}_{2} \mathbb{F}_{p}\right| \\
& =p^{3}-p
\end{aligned}
$$

Finally, suppose that $(D / p)=0$. This means that $p$ ramifies in $\mathcal{O}_{D}$, so $\mathcal{O}_{D} / p \cong$ $\mathbb{F}_{p}(\epsilon) /\left(\epsilon^{2}\right)$. We have

$$
\left(\begin{array}{ll}
a+a^{\prime} \epsilon & b+b^{\prime} \epsilon \\
c+c^{\prime} \epsilon & d+d^{\prime} \epsilon
\end{array}\right) \in \mathrm{SL}_{2}\left(\mathbb{F}_{p}(\epsilon) /\left(\epsilon^{2}\right)\right)
$$

if and only if

$$
\begin{gathered}
a d-b c=1, \\
d a^{\prime}+a d^{\prime}-c b^{\prime}-b c^{\prime}=0 .
\end{gathered}
$$

For any $(a, b, c, d)$ satisfying (2-9), there are $p^{3}$ solutions to (2-10). Thus,

$$
\left[\mathrm{SL}_{2}\left(\mathbb{F}_{p}(\epsilon) /\left(\epsilon^{2}\right)\right): \mathrm{SL}_{2} \mathbb{F}_{p}\right]=p^{3} .
$$

Proof of Theorem 2.12 Since the natural map $X_{f^{2} D} \rightarrow X_{D}$ is an orbifold covering map, we have

$$
\chi\left(X_{f^{2} D}\right)=\chi\left(X_{D}\right)\left[\mathrm{PSL}_{2} \mathcal{O}_{D}: \mathrm{PSL}_{2} \mathcal{O}_{f^{2} D}\right]
$$

so the claim follows from Lemma 2.13, Lemma 2.14, and Theorem 2.11 together with

$$
\chi\left(X_{1}\right)=\frac{1}{36}
$$

because $X_{1} \cong \mathbb{H} / \mathrm{SL}_{2} \mathbb{Z} \times \mathbb{H} / \mathrm{SL}_{2} \mathbb{Z}$, and

$$
\chi\left(\mathbb{H} / \mathrm{SL}_{2} \mathbb{Z}\right)=-\frac{1}{6} .
$$


Modular forms For any fundamental discriminant $D>0$, define

$$
H\left(2, f^{2} D\right)=-12 \zeta_{K_{D}}(-1) \sum_{r \mid f} \mu(r)\left(\frac{D}{r}\right) r \sigma_{3}\left(\frac{f}{r}\right),
$$

where

$$
\sigma_{m}(n)=\sum_{d \mid n} d^{m}
$$

and we also adopt the convention that

$$
\sigma_{m}(0)=\frac{1}{2} \zeta_{\mathbb{Q}}(-m),
$$

and $\sigma_{m}(n)=0$ if $n<0$. Also define

$$
H(2,0)=\zeta_{\mathbb{Q}}(-3)=\frac{1}{120} .
$$

The function $\mathcal{H}: \mathbb{W} \rightarrow \mathbb{C}$,

$$
\begin{aligned}
\mathcal{H}(\tau) & =\sum_{\substack{D \equiv 0,1(4) \\
D \geq 0}} H(2, D) q^{D}, \quad\left(\text { with } q(\tau)=e^{2 \pi i \tau}\right) \\
& =-\frac{1}{120}-\frac{1}{12} q-\frac{7}{12} q^{4}-\frac{2}{5} q^{5}-q^{8}-\frac{25}{12} q^{9}-2 q^{12}-2 q^{13}-\frac{55}{12} q^{16}-\ldots,
\end{aligned}
$$

was shown by Cohen in [19], to be a modular form of weight $5 / 2$ for the group $\Gamma_{0}(4)$. See the discussion in Chapter IX of [75] for more about this form and its relation to $X_{D}$.

It is an elementary argument using Möbius inversion to show that for any fundamental discriminant $D$ and $f \in \mathbb{N}$,

$$
H\left(2, f^{2} D\right)=-12 \sum_{s \mid f}\left(\zeta_{K_{D}}(-1) s^{3} \sum_{r \mid s}\left(\frac{D}{r}\right) \frac{\mu(r)}{r^{2}}\right) .
$$

From this and Theorem 2.12, we obtain:

Theorem 2.15 If $D \neq 1$ is a fundamental discriminant, then

$$
\sum_{r \mid f} \chi\left(X_{r^{2} D}\right)=-\frac{1}{6} H\left(2, f^{2} D\right)
$$

Siegel and Cohen gave the following simple formula for $H(2, D)$. 
Theorem 2.16 [74; 19] If $D$ is not square, then

$$
H(2, D)=-\frac{1}{5} \sum_{e \equiv D(2)} \sigma_{1}\left(\frac{D-e^{2}}{4}\right) .
$$

When $D$ is square,

$$
H(2, D)=-\frac{1}{5} \sum_{e \equiv D(2)} \sigma_{1}\left(\frac{D-e^{2}}{4}\right)-\frac{D}{10} .
$$

We will obtain an alternative proof of these formulas in Section 11.

The product locus In $X_{D}$, define the product locus $P_{D}$ to be the set of all $A \in X_{D}$ such that $A$ is a polarized product of elliptic curves. The locus $P_{D}$ was studied in [54] and [58]. The locus $P_{D}$ is also a union of modular curves, which were studied in [34] and [75].

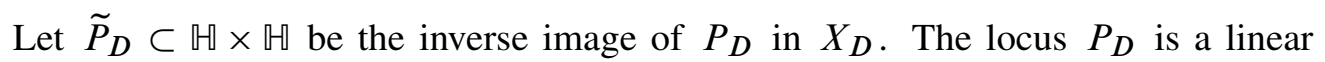
subvariety of $X_{D}$ in the following sense.

Proposition 2.17 [54] $\widetilde{P}_{D}$ is a countable union of graphs of Möbius transformations $\mathbb{H} \rightarrow \mathbb{H}$.

The goal of the rest of this section is to calculate $\chi\left(P_{D}\right)$. We will work with an auxiliary covering space $Q_{D}$ of $P_{D}$ defined as follows.

Let $\Omega Q$ be the space of pairs of elliptic curves equipped with Abelian differentials; that is,

$$
\Omega Q=\Omega \mathcal{A}_{1} \times \Omega \mathcal{A}_{1}=\mathrm{GL}_{2}^{+} \mathbb{R} / \mathrm{SL}_{2} \mathbb{Z} \times \mathrm{GL}_{2}^{+} \mathbb{R} / \mathrm{SL}_{2} \mathbb{Z},
$$

where $\mathrm{GL}_{2}^{+} \mathbb{R}$ is the subgroup of $\mathrm{GL}_{2} \mathbb{R}$ consisting of matrices of positive determinant. In $\Omega Q$, let $\Omega Q_{D}$ be the locus of pairs $\left(\left(E_{1}, \omega_{1}\right),\left(E_{2}, \omega_{2}\right)\right)$ such that the product

$$
\left(E_{1}, \omega_{1}\right) \oplus\left(E_{2}, \omega_{2}\right):=\left(E_{1} \times E_{2}, \omega_{1}+\omega_{2}\right)
$$

is an eigenform for real multiplication by $\mathcal{O}_{D}$. The projectivization,

$$
Q_{D}=\mathbb{P} \Omega Q_{D},
$$

is a union of curves.

We now describe the connected components of $\Omega Q_{D}$ following [54]. A prototype for real multiplication by $\mathcal{O}_{D}$ is a triple of integers $(e, l, m)$ such that

$$
D=e^{2}+4 l^{2} m, \quad l, m>0, \quad \text { and } \quad(e, l)=1 .
$$


To each such prototype, we associate a prototypical eigenform $\mathcal{Q}(e, l, m)$ as follows. Let $\lambda$ be the unique positive root of $\lambda^{2}-e \lambda-l^{2} m=0$, and consider the lattices in $\mathbb{C}$

$$
\begin{aligned}
& \Lambda_{1}=\mathbb{Z}(\lambda, 0) \oplus \mathbb{Z}(0, \lambda), \\
& \Lambda_{2}=\mathbb{Z}(l m, 0) \oplus \mathbb{Z}(0, l) .
\end{aligned}
$$

Let $E_{i}=\mathbb{C} / \Lambda_{i}$, and equip $E_{i}$ with the form $\omega_{i}$ covered by $d z$ on $\mathbb{C}$. Let

$$
\mathcal{Q}(e, l, m)=\left(E_{1}, \omega_{1}\right) \oplus\left(E_{2}, \omega_{2}\right) .
$$

From the description of $\Omega Q$ in (2-12), we obtain an action of $\mathrm{GL}_{2}^{+} \mathbb{R}$ on $\Omega Q$ induced by the diagonal action of $\mathrm{GL}_{2}^{+} \mathbb{R}$ on $\mathrm{GL}_{2}^{+} \mathbb{R} \times \mathrm{GL}_{2}^{+} \mathbb{R}$.

Theorem 2.18 [54, Theorem 2.1] The locus $\Omega_{D}$ is invariant under the action of $\mathrm{GL}_{2}^{+} \mathbb{R}$. Each component is a $\mathrm{GL}_{2}^{+} \mathbb{R}$ orbit which contains exactly one prototypical eigenform $\mathcal{Q}(e, l, m)$ and is isomorphic to $\mathrm{GL}_{2}^{+} \mathbb{R} / \Gamma_{0}(m)$.

Remark Here $\Gamma_{0}(m)$ is the congruence subgroup of $\mathrm{SL}_{2} \mathbb{Z}$ defined by

$$
\Gamma_{0}(m)=\left\{\left(\begin{array}{ll}
a & b \\
c & d
\end{array}\right) \in \mathrm{SL}_{2} \mathbb{Z}: c \equiv 0 \quad(\bmod m)\right\} .
$$

Theorem 2.19 For any nonsquare discriminant $D$, we have

$$
\chi\left(Q_{D}\right)=-5 \chi\left(X_{D}\right) .
$$

If $D=d^{2}$ with $d \geq 2$, then we have

$$
\chi\left(Q_{d^{2}}\right)=-\frac{1}{72} d^{2}(5 d-6) \sum_{r \mid d} \frac{\mu(r)}{r^{2}} .
$$

Proof We first claim that

$$
\sum_{s \mid f} \chi\left(Q_{s^{2} D}\right)=-\frac{1}{6} \sum_{\substack{e \equiv f^{2} D(2) \\-f \sqrt{D}<e<f \sqrt{D}}} \sigma_{1}\left(\frac{f^{2} D-e^{2}}{4}\right)
$$

for any fundamental discriminant $D$ and $f \in \mathbb{N}$. By Theorem 2.18, there is one component of $Q_{D}$ for each prototype $(e, l, m)$ which is isomorphic to $\mathbb{E} / \Gamma_{0}(m)$. By [59, Theorem 4.2.5],

$$
\chi\left(\mathbb{H} / \Gamma_{0}(m)\right)=\psi(m),
$$

where

$$
\psi(m)=-\frac{1}{6} m \prod_{\substack{p \mid m \\ p \text { prime }}}\left(1+\frac{1}{p}\right) .
$$


It is elementary to show that for any $n \in \mathbb{N}$,

$$
-\frac{1}{6} \sigma_{1}(n)=\sum_{\substack{n=l^{2} m \\ l, m \in \mathbb{N}}} \psi(m) .
$$

By Theorem 2.18,

$$
\begin{aligned}
& \sum_{r \mid f} \chi\left(Q_{f^{2} D}\right)= \sum_{\substack{f^{2} D=e^{2}+4 l^{2} m \\
l, m>0}} \psi(m) \\
&=-\frac{1}{6} \sum_{\substack{e \equiv f^{2} D(2) \\
-f \sqrt{D<e<f \sqrt{D}}}} \sigma_{1}\left(\frac{f^{2} D-e^{2}}{4}\right),
\end{aligned}
$$

which proves (2-17).

If $D \neq 1$ is a fundamental discriminant, then for any $f \in \mathbb{N}$, we have

$$
\sum_{r \mid f} \chi\left(Q_{r^{2} D}\right)=\frac{5}{6} H\left(2, f^{2} D\right)=-5 \sum_{r \mid f} \chi\left(X_{r^{2} D}\right)
$$

by Theorem 2.16 and (2-17), from which (2-15) follows by Möbius inversion.

Now for any $d \in \mathbb{N}$, we have

$$
\frac{1}{72}+\sum_{\substack{r \mid d \\ r>1}} \chi\left(Q_{r^{2}}\right)=\frac{5}{6} H\left(2, d^{2}\right)+\frac{d^{2}}{12}
$$

by Theorem 2.16 and (2-17), and using the convention that $\sigma_{1}(0)=-1 / 24$. From (2-11), we get for any $d \in \mathbb{N}$,

$$
H\left(2, d^{2}\right)=\sum_{s \mid d}\left(-\frac{1}{12} s^{3} \sum_{r \mid s} \frac{\mu(r)}{r^{2}}\right) .
$$

It follows from Möbius inversion that for any $d \in \mathbb{N}$,

$$
d^{2}=\sum_{s \mid d}\left(s^{2} \sum_{r \mid s} \frac{\mu(r)}{r^{2}}\right) .
$$

Define

$$
f(d)= \begin{cases}1 / 72 & \text { if } d=1 \\ \chi\left(Q_{d^{2}}\right) & \text { if } d>1\end{cases}
$$


Combining (2-18), (2-19), and (2-20), we obtain

$$
\sum_{s \mid d} f(s)=\sum_{s \mid d}\left(\left(-\frac{5}{72} s^{3}+\frac{1}{12} s^{2}\right) \sum_{r \mid s} \frac{\mu(r)}{r^{2}}\right),
$$

which implies (2-16) by Möbius inversion.

There is an involution of $\Omega Q_{D}$ which interchanges the order of the two factors in (2-12). This involution preserves $\Omega Q_{D}$ and so restricts to an involution $\tau$ of $\Omega_{D}$. The involution $\tau$ preserves the fibers of the bundle $\Omega_{D} \rightarrow Q_{D}$, so it induces an involution $\bar{\tau}$ of $Q_{D}$.

Lemma 2.20 The involution $\bar{\tau}$ fixes pointwise the component of $Q_{D}$ containing the prototypical eigenform $\mathcal{Q}(e, l, m)$ if and only if

$$
\bar{\tau} \mathcal{Q}(e, l, m)=\mathcal{Q}(e, l, m) .
$$

The only such component on which $\tau$ is the identity is the one containing $\mathcal{Q}(0,1,1)$.

Proof First, note that (2-21) holds if and only if $\Lambda_{1}=\Lambda_{2}$ in (2-14), which is true if and only if $m=1$ and $\lambda=l$. The only prototype for which this holds is $(0,1,1)$, so $(2-21)$ is true if and only if $(e, l, m)=(0,1,1)$.

Clearly, (2-21) must hold for $\bar{\tau}$ to be the identity on the component containing $\mathcal{Q}(e, l, m)$. Conversely, if $(2-21)$ does hold, then $(e, l, m)=(0,1,1)$, and

$$
\tau(g \cdot \mathcal{Q}(0,1,1))=g \cdot \mathcal{Q}(0,1,1)
$$

for any $g \in \mathrm{GL}_{2}^{+} \mathbb{R}$ because $\tau$ commutes with the action of $\mathrm{GL}_{2}^{+} \mathbb{R}$ on $\Omega Q_{D}$. Thus $\tau$ fixes every point of the $\mathrm{GL}_{2}^{+} \mathbb{R}$-orbit of $\mathcal{Q}(0,1,1)$, which is exactly the component of $\Omega Q_{4}$ containing $\mathcal{Q}(0,1,1)$.

There is natural map $\pi: \Omega Q_{D} \rightarrow \Omega P_{D}$ defined by

$$
\pi\left(\left(E_{1}, \omega_{1}\right),\left(E_{2}, \omega_{2}\right)\right)=\left(E_{1}, \omega_{1}\right) \oplus\left(E_{2}, \omega_{2}\right)
$$

which descends to a map $\bar{\pi}: Q_{D} \rightarrow P_{D}$.

Lemma 2.21 The map $\bar{\pi}: Q_{D} \rightarrow P_{D}$ factors through to an isomorphism of orbifolds, $\tilde{\pi}: Q_{D} / \bar{\tau} \rightarrow P_{D}$.

Proof The map $\bar{\pi}$ factors because the right hand side of (2-22) does not depend on the order of the $E_{i}$. It is clearly onto. That $\bar{\pi}$ is one-to-one follows from the following fact about Abelian surfaces: a principally polarized Abelian surface can have at most one representation as a polarized product of elliptic curves. 
Theorem 2.22 If $D$ is not square, then

$$
\chi\left(P_{D}\right)=-\frac{5}{2} \chi\left(X_{D}\right)
$$

If $D=d^{2}$, then

$$
\chi\left(P_{d^{2}}\right)=-\frac{1}{144} d^{2}(5 d-6) \sum_{r \mid d} \frac{\mu(r)}{r^{2}}
$$

when $d>2$, and

$$
\chi\left(P_{4}\right)=-\frac{1}{6}
$$

Remark The formula (2-23) was proved previously by Kani [37; 38] and Schmoll [70].

Proof By Theorem 2.18, $Q_{4}$ consists of a single component containing $\mathcal{Q}(0,1,1)$. Thus by Lemma 2.20 and Lemma 2.21, $P_{4} \cong Q_{4}$, and $Q_{D}$ is a twofold cover of $P_{D}$ when $D>4$. The claim then follows from Theorem 2.19.

\section{Prototypes}

\section{1 $P_{D}, W_{D}$, and $Y_{D}$-prototypes}

Curves in $Y_{D} \backslash X_{D}$ will be classified by certain discrete numerical invariants which we call $Y_{D}$-prototypes. They are almost (but not quite) the same as the splitting prototypes introduced by McMullen in [54] to classify the cusps of $W_{D}$.

Definition A $Y_{D}$-prototype of discriminant $D$ is a quadruple $(a, b, c, \bar{q})$ with $a, b, c \in$ $\mathbb{Z}$ and $\bar{q} \in \mathbb{Z} / \operatorname{gcd}(a, b, c)$ which satisfies the following six properties:

(1) $b^{2}-4 a c=D$

(2) $a>0$

(3) $c \leq 0$

(4) $\operatorname{gcd}(a, b, c, \bar{q})=1$

(5) $a+b+c \leq 0$

(6) $a+b+c$ and $c$ are not both zero. 
Let $\mathcal{Y}_{D}$ be the set of $Y_{D}$-prototypes. Define a map $\lambda: \mathcal{Y}_{D} \rightarrow K_{D}$ by associating to each prototype $P=(a, b, c, \bar{q})$ of discriminant $P$ the unique algebraic number $\lambda(P) \in K_{D}$ such that $a \lambda(P)^{2}+b \lambda(P)+c=0$ and $\lambda^{(1)}>0$. This makes sense because if $c<0$, then the two roots of $a x^{2}+b x+c=0$ have opposite signs, and if $c=0$, then the condition that $a+b<0$ implies that the nonzero root of $a \lambda^{2}+b \lambda=0$ is positive. It is easy to check that the last condition $a+b+c \leq 0$ is equivalent to $\lambda^{(1)} \geq 1$.

We say that a prototype is terminal if $a+b+c=0$; it is initial if $a-b+c=0$; and it is degenerate if $c=0$. Terminal, initial, and degenerate prototypes only arise if $D$ is square. We define the involution,

$$
(a, b, c, \bar{q}) \mapsto(-c,-b,-a, \bar{q}),
$$

on the set of terminal prototypes. We consider two terminal prototypes to be the same if they are related by this involution. Similarly, we consider two degenerate prototypes to be the same if they are related by the involution,

$$
(a, b, 0, \bar{q}) \mapsto(-b-a, b, 0, \bar{q}),
$$

on the set of degenerate prototypes.

Operations on $\boldsymbol{Y}_{\boldsymbol{D}}$-prototypes Given a nonterminal prototype $P$, define the next prototype $P^{+}$by

$$
P^{+}= \begin{cases}(a, 2 a+b, a+b+c, \bar{q}), & \text { if } 4 a+2 b+c \leq 0 \\ (-a-b-c,-2 a-b,-a, \bar{q}), & \text { if } 4 a+2 b+c \geq 0\end{cases}
$$

Given a nondegenerate prototype $P$, define the previous prototype $P^{-}$by

$$
P^{-}= \begin{cases}(a,-2 a+b, a-b+c, \bar{q}), & \text { if } a-b+c \leq 0 \\ (-c,-b+2 c,-a+b-c, \bar{q}), & \text { if } a-b+c \geq 0\end{cases}
$$

Its easy to check that $P^{+}$and $P^{-}$are actually prototypes of the same discriminant and that

$$
\left(P^{+}\right)^{-}=\left(P^{-}\right)^{+}=P
$$

when these operations are defined. 
On the level of $\lambda(P)$, we have

and

$$
\begin{aligned}
& \lambda\left(P^{+}\right)= \begin{cases}\lambda(P)-1, & \text { if } \lambda(P)^{(1)}-1 \geq 1 ; \\
1 /(\lambda(P)-1), & \text { if } \lambda(P)^{(1)}-1<1 ;\end{cases} \\
& \lambda\left(P^{-}\right)= \begin{cases}\lambda(P)+1, & \text { if } N_{\mathbb{Q}}^{K_{D}}(\lambda(P)+1) \leq 0 ; \\
(\lambda(P)+1) / \lambda(P), & \text { if } N_{\mathbb{Q}}^{K_{D}}(\lambda(P)+1)>0 .\end{cases}
\end{aligned}
$$

Define an involution $t$ on the set of prototypes of discriminant $D$ by

$$
t(a, b, c, \bar{q})= \begin{cases}(a,-b, c, \bar{q}), & \text { if } a-b+c \leq 0 \\ (-c, b,-a, \bar{q}), & \text { if } a-b+c \geq 0\end{cases}
$$

Define the multiplicity of a nondegenerate prototype $P$ by

$$
\operatorname{mult}(P)=\frac{\operatorname{gcd}(a, c)}{\operatorname{gcd}(a, b, c)}
$$

$\boldsymbol{P}_{\boldsymbol{D}}$ and $\boldsymbol{W}_{\boldsymbol{D}}$-prototypes In addition to the $Y_{D}$-prototypes, we will define similar objects which we call $P_{D}$ and $W_{D}$-prototypes. These will classify the cusps of the curves $P_{D}$ and $W_{D}$.

Definition A $P_{D}$-prototype of discriminant $D$ is a quadruple, $(a, b, c, \bar{q})$ with $a, b, c \in \mathbb{Z}$ and $\bar{q} \in \mathbb{Z} / \operatorname{gcd}(a, c)$ which satisfies the following five properties:

(1) $b^{2}-4 a c=D$

(2) $a>0$

(3) $c<0$

(4) $\operatorname{gcd}(a, b, c, \bar{q})=1$

(5) $a+b+c \leq 0$

Note that the only difference between $Y_{D}$ and $P_{D}$-prototypes are that for $P_{D^{-}}$ prototypes, $\bar{q}$ ranges in a potentially larger group, and $c$ is not allowed to be zero.

A $W_{D}$-prototype of discriminant $D$ is a quadruple, $(a, b, c, \bar{q})$ with $a, b, c \in \mathbb{Z}$ and $\bar{q} \in \mathbb{Z} / \operatorname{gcd}(a, c)$ which satisfies the same properties as above except that the last one is replaced with

$$
a+b+c<0 .
$$

Let $\mathcal{P}_{D}$ and $\mathcal{W}_{D}$ be the sets of $P_{D}$ and $W_{D}$-prototypes. Just as for the $Y_{D}$-prototypes, we have natural maps $\lambda: \mathcal{W}_{D} \rightarrow K_{D}$ and $\lambda: \mathcal{P}_{D} \rightarrow K_{D}$. By reducing $\bar{q}$ modulo $\operatorname{gcd}(a, b, c)$, there are natural maps $\mathcal{P}_{D} \rightarrow \mathcal{Y}_{D}$ and $\mathcal{W}_{D} \rightarrow \mathcal{Y}_{D}$.

We will see in Section 8.1 that these maps encode intersections of cusps of $W_{D}$ and $P_{D}$ with curves in $Y_{D} \backslash X_{D}$. 
Relation with splitting prototypes In [54], McMullen defined a splitting prototype to be a quadruple of integers $(a, b, c, e)$ satisfying certain properties. Splitting prototypes correspond bijectively to our $W_{D}$-prototypes by sending the splitting prototype $(a, b, c, e)$ to the $W_{D}$-prototype $(c, e,-b, \bar{a})$.

\subsection{Quasi-invertible $\mathcal{O}_{D}$-modules}

In this section, we will study a class of $\mathcal{O}_{D}$-modules which arises naturally in the study of our compactification of the Hilbert modular surface. We will also study a class of bases of these modules whose combinatorics is closely related to the geometry of the compactification. This material will only be used in Section 7.

Definition An $\mathcal{O}_{D}$-module $M$ is quasi-invertible if $M \cong \mathbb{Z} \oplus \mathbb{Z}$ as an Abelian group, and $M$ contains some element $x$ such that $\operatorname{Ann}(x)=0$.

Examples If $D$ is a fundamental discriminant, then every quasi-invertible $\mathcal{O}_{D}$-module is actually invertible. Since $\mathcal{O}_{D}$ is a Dedekind domain, the set of isomorphism classes of such modules forms a group, the ideal class group of $\mathcal{O}_{D}$.

If $D$ is not square, then a quasi-invertible $\mathcal{O}_{D}$-module is just an invertible $\mathcal{O}_{E}$-module over some order $\mathcal{O}_{E}$ containing $\mathcal{O}_{D}$. A quasi-invertible $\mathcal{O}_{D}$-module is invertible if and only if it is primitive in the sense that it is not also a module over any $\mathcal{O}_{E}$ containing $\mathcal{O}_{D}$

In this paper, quasi-invertible $\mathcal{O}_{D}$-modules will arise in the following way:

Proposition 3.1 If $M \subset \mathcal{O}_{D} \oplus \mathcal{O}_{D}^{\vee}$ is a Lagrangian $\mathcal{O}_{D}$-submodule which has $\mathbb{Z}$-rank two, then $M$ is quasi-invertible.

Proof This is trivial if $D$ is not square because then $\mathcal{O}_{D} \oplus \mathcal{O}_{D}^{\vee}$ is torsion-free, so suppose $D=d^{2}$. Let $M \subset \mathcal{O}_{d^{2}} \oplus \mathcal{O}_{d^{2}}^{\vee}$ be a rank two Lagrangian submodule which is not quasi-invertible, and let $\left\{u_{1}, u_{2}\right\}$ be a basis of $M$. We must then have either $(d, 0) \cdot u_{i}=0$ for $i=1,2$, or $(0, d) \cdot u_{i}=0$ for $i=1,2$, or else there would be some $x \in M$ such that $\operatorname{Ann}(x)=0$. For concreteness, suppose we are in the second case. Then we must have

$$
u_{i}=\left(\left(a_{i}, 0\right),\left(b_{i}, 0\right)\right)
$$

for some $a_{1}, b_{i} \in \mathbb{Q}$.

Since $M$ is Lagrangian, we know that

$$
\begin{aligned}
0=\left\langle u_{1}, u_{2}\right\rangle & =\operatorname{Tr}_{\mathbb{Q}}^{\mathbb{Q} \oplus \mathbb{Q}}\left|\begin{array}{ll}
\left(a_{1}, 0\right) & \left(a_{2}, 0\right) \\
\left(b_{1}, 0\right) & \left(b_{2}, 0\right)
\end{array}\right| \\
& =a_{1} b_{2}-a_{2} b_{2},
\end{aligned}
$$


so $u_{1}=r u_{2}$ for some $r \in \mathbb{Q}$. This contradicts the fact that the $u_{i}$ are a basis.

Embeddings in $K_{D}$ A lattice in $K_{D}$ is a rank two Abelian subgroup. Given a lattice $M \subset K_{D}$, the set

$$
\left\{x \in K_{D}: x M \subset M\right\}
$$

is called the coefficient ring of $M$. It is an order in $K_{D}$.

Proposition 3.2 An $\mathcal{O}_{D}$-module $M$ is quasi-invertible if and only if it is isomorphic to some lattice $M \subset K_{D}$ whose coefficient ring contains $\mathcal{O}_{D}$.

If $M$ is quasi-invertible, then the embedding $M \rightarrow K_{D}$ is unique up to multiplication by a non-zero divisor in $K_{D}$.

Proof If $D$ is not square, then it is clear that a lattice $M \subset K_{D}$ whose coefficient ring contains $\mathcal{O}_{D}$ is a quasi-invertible $\mathcal{O}_{D}$-module because $M$ is automatically torsion-free. If $D=d^{2}$, then $M$ could have $\operatorname{Ann}(x) \neq 0$ for every $x \in M$ only if $M \subset \mathbb{Q} \oplus\{0\}$ or $M \subset\{0\} \oplus \mathbb{Q}$. This cannot happen because $M$ has rank two.

Conversely, suppose $M$ is a quasi-invertible $\mathcal{O}_{D}$-module with $x \in M$ such that $\operatorname{Ann}(x)=0$. Since $M$ is torsion-free as an Abelian group, the natural map $M \rightarrow M \otimes \mathbb{Q}$ is injective. The tensor product $M \otimes \mathbb{Q}$ is a $K_{D}$-module and a two-dimensional vector space over $\mathbb{Q}$, so the map $K_{D} \rightarrow M \otimes \mathbb{Q}$ defined by $r \mapsto r \cdot x$ is an isomorphism. The inverse of this map embeds $M$ as a lattice whose coefficient ring contains $\mathcal{O}_{D}$. Since the image of $x$ determined the embedding, it is unique up to constant multiple.

It is easy to identify the coefficient ring of a lattice in $K_{D}$. Let $M_{\lambda}$ be the lattice generated by $\{1, \lambda\}$. For $\lambda \in K_{D}$, let $\phi_{\lambda}(t)$ be the minimal polynomial of $\lambda$, the unique polynomial $\phi_{\lambda}(t)=a t^{2}+b t+c$ such that $a, b, c \in \mathbb{Z}, a>0, \phi_{\lambda}(\lambda)=0$, and $\operatorname{gcd}(a, b, c)=1$.

Proposition $3.3[14, \mathrm{p} 136]$ If $\lambda \in K_{D} \backslash \mathbb{Q}$ with $\phi_{\gamma}(t)=a t^{2}+b t+c$, then the coefficient ring of $M_{\lambda}$ is the order $\mathbb{Z}[a \lambda]$, which is isomorphic to $\mathcal{O}_{D}$, where $D=b^{2}-4 a c$.

Admissible bases Since a quasi-invertible $\mathcal{O}_{D}$-module $M$ can be embedded in $K_{D}$ uniquely up to constant multiple, for any $u, v \in M$ with $\operatorname{Ann}(v) \neq 0$, the ratio $u / v$ is a well-defined element of $K_{D}$.

Definition A basis $\{u, v\}$ of a quasi-invertible $\mathcal{O}_{D}$-module $M$ is admissible if $\operatorname{Ann}(v)=0$ and $N_{\mathbb{Q}}^{K_{D}}(u / v)<0$. 
We will consider two admissible bases of $M$ to be equivalent if they are the same as subsets of $M / \pm 1$. The following proposition gives a complete classification of quasi-invertible $\mathcal{O}_{D}$-modules together with an admissible basis.

Proposition 3.4 Every pair $(M,\{u, v\})$, where $M$ is a quasi-invertible $\mathcal{O}_{D}$-module together with an admissible basis $\{u, v\}$, is equivalent to one of the form $\left(M_{\lambda},\{1, \lambda\}\right)$, where $\lambda^{(1)} \geq 1$ and $\psi(\lambda)=0$, where

$$
\psi(t)=a t^{2}+b t+c
$$

for some integers $(a, b, c)$ such that:

(1) $b^{2}-4 a c=D$

(2) $a>0$

(3) $c<0$

(4) $a+b+c \leq 0$

(5) $\mathcal{O}_{D}=\mathbb{Z}[a \lambda]$

Pairs $\left(M_{\lambda},\{1, \lambda\}\right)$ and $\left(M_{\mu},\{1, \mu\}\right)$ of this form are equivalent if and only if $\lambda=\mu^{-1}$.

Proof If $u^{(1)} / v^{(1)} \neq \pm 1$, then by Proposition 3.2, there is a unique embedding $M \rightarrow K_{D}$ which - after possibly switching $u$ and $v$ and changing their signs - sends $u$ to 1 and $v$ to some $\lambda \in K_{D}$ with $\lambda^{(1)}>1$. The coefficient ring of the image $M_{\lambda}$ is $\mathcal{O}_{E}$, where $D=s^{2} E$ for some quadratic discriminant $E$. Let $\phi_{\lambda}(t)=a^{\prime} t^{2}+b^{\prime} t+c^{\prime}$ be the minimal polynomial of $\lambda$. By Proposition 3.3, we have $b^{\prime 2}-4 a^{\prime} c^{\prime}=E$ and $\mathcal{O}_{E}=\mathbb{Z}\left[a^{\prime} \lambda\right]$. We have $c^{\prime}<0$ because $N_{\mathbb{Q}}^{K_{D}}(u / v)<0$. Since $\lambda^{(1)}>1$, we know that $a^{\prime}+b^{\prime}+c^{\prime}<0$. The integers $(a, b, c)=s\left(a^{\prime}, b^{\prime}, c^{\prime}\right)$ then have the required properties.

If $u^{(1)} / v^{(1)}= \pm 1$, then there are two embeddings $M \rightarrow K_{D}$ as above, one sending $u$ to 1 and $v$ to $\lambda$; the other sending $u$ to $\lambda^{-1}$ and $v$ to 1 . As in the above paragraph, both embeddings yield a presentation of $(M,\{u, v\})$ in the desired form.

We can define a map from the set of nondegenerate $Y_{D}$-prototypes to the set of isomorphism classes of admissible bases of quasi-invertible $\mathcal{O}_{D}$-modules by sending $P$ to $\left(M_{\lambda(P)},\{1, \lambda(P)\}\right)$. This proposition implies that this map is onto and that two prototypes are sent to the same admissible basis if and only if their integers $a, b$, and $c$ are the same. 
Characterization of admissible bases We now describe a useful characterization of admissible bases. Given two quasi-invertible $\mathcal{O}_{D}$-modules $M$ and $N$, a perfect pairing is a bilinear map

$$
h: M \times N \rightarrow \mathbb{Z}
$$

such that

$$
h(\lambda \cdot x, y)=h(x, \lambda \cdot y)
$$

for each $x \in M, y \in N$, and $\lambda \in \mathcal{O}_{D}$, and such that the induced map $M \rightarrow \operatorname{Hom}(N, \mathbb{Z})$ is an isomorphism.

Given such a perfect pairing and a basis $\{u, v\}$ of $M$, let $\left\{u^{*}, v^{*}\right\}$ be a dual basis of $N$, and define

$$
\operatorname{sign}(u, v ; h)=\operatorname{sign}\left(\iota_{M}(u) \iota_{M}(v) \iota_{N}\left(u^{*}\right) \iota_{N}\left(v^{*}\right)\right),
$$

where $\iota_{M}$ and $\iota_{N}$ are $\iota_{1}$-linear, nonzero maps $M \rightarrow \mathbb{R}$ and $N \rightarrow \mathbb{R}$. Note that $\operatorname{sign}(u, v ; h)$ is invariant under changing the sign of $u$ or $v$ as well as independent of the choice of $\iota_{M}$ and $\iota_{N}$.

Theorem 3.5 Given a quasi-invertible $\mathcal{O}_{D}$-module $M$, a basis $\{u, v\}$ is an admissible basis if and only if $\operatorname{sign}(u, v ; h)=1$ for some (or any) perfect pairing $h: M \times N \rightarrow \mathbb{Z}$.

Proof Assume that $M$ is embedded in $K_{D}$. If either $u^{(1)}=0$ or $v^{(1)}=0$, then $\operatorname{sign}(u, v ; h)=0$, and the basis is not admissible, so we are done. Since $M$ is quasiinvertible, it can't happen that $u^{(2)}=v^{(2)}=0$, so assume $u^{(2)} \neq 0$.

Assume without loss of generality that $u=1$. By possibly changing the sign of $v$, we can suppose $v^{(1)}>0$. Then $v^{(2)}<0$ if and only if $\{u, v\}$ is admissible. Since $N$ and the perfect pairing $h$ are unique up to isomorphism, we can suppose without loss of generality that $N=M^{\vee}$, the inverse different of $M$, and that $h$ is the trace pairing $M \times M^{\vee} \rightarrow \mathbb{Z}$.

Define

$$
\begin{aligned}
u^{*} & =\frac{-v^{\prime}}{v-v^{\prime}} \\
v^{*} & =\frac{1}{v-v^{\prime}} .
\end{aligned}
$$

It is easy to check that $\left\{u^{*}, v^{*}\right\}$ is dual to $\{u, v\}$ with respect to the trace pairing. If $\{u, v\}$ is admissible, then $v^{(2)}<0$, in which case $\left(u^{*}\right)^{(1)},\left(v^{*}\right)^{(1)}>0$, $\operatorname{so} \operatorname{sign}(u, v ; h)>$ 0 . If $\{u, v\}$ is not admissible, then $v^{(2)} \geq 0$. If $v^{(2)}>0$, then $\left(u^{*}\right)^{(1)}$ and $\left(v^{*}\right)^{(1)}$ have opposite signs, so $\operatorname{sign}(u, v ; h)=-1$. If $v^{(2)}=0$, then $\left(u^{*}\right)^{(1)}=0$, $\operatorname{so} \operatorname{sign}(u, v ; h)=$ 0 . 
Admissible triples We are also interested in special triples of elements of quasiinvertible $\mathcal{O}_{D}$-modules which will arise naturally in the study of compactifications of Hilbert modular surfaces.

Definition An admissible triple in a quasi-invertible $\mathcal{O}_{D}$-module $M$ is an unordered triple of elements $\{a, b, c\} \subset M / \pm 1$ such that:

- $\pm a \pm b \pm c=0$ for some choice of signs.

- Some pair of elements of the triple form an admissible basis of $M$.

Proposition 3.6 Every pair $(M, T)$, where $M$ is a quasi-invertible $\mathcal{O}_{D}$-module with an admissible triple $T \subset M$, is equivalent to one of the form

$$
\left(M_{\lambda},\{1, \lambda, \lambda-1\}\right),
$$

where $\lambda^{(1)} \geq 1$ and

$$
a \lambda^{2}+b \lambda+c=0
$$

for some integers $(a, b, c)$ such that:

(1) $b^{2}-4 a c=D$

(2) $a>0$

(3) $c \leq 0$

(4) $a+b+c \leq 0$

(5) $\mathcal{O}_{D}=\mathbb{Z}[a \lambda]$

(6) $a+b+c$ and $c$ are not both zero.

Two such pairs $\left(M_{\lambda},\{1, \lambda, \lambda-1\}\right)$ and $\left(M_{\mu},\{1, \mu, \mu-1\}\right)$ with $\mu \neq \lambda$ are equivalent if and only if $\lambda^{(1)}=1$ and $\mu=\lambda^{-1}$ or if $\lambda^{(2)}=0$ and $\mu=\lambda /(\lambda-1)$.

Proof Let $\{u, v, w\} \subset M$ be an admissible triple and assume that $M$ is embedded in $K_{D}$. This triple contains at least one admissible basis, so assume without loss of generality that $\{u, v\}$ is an admissible basis. There are now three cases to consider, depending on whether $\operatorname{Ann}(w)=0, w^{(1)}=0$, or $w^{(2)}=0$.

First suppose that $\operatorname{Ann}(w)=0$. Then $\{u, v, w\}$ contains exactly two admissible bases because

$$
N_{\mathbb{Q}}^{K_{D}}\left(\frac{u}{v}\right) N_{\mathbb{Q}}^{K_{D}}\left(\frac{v}{w}\right) N_{\mathbb{Q}}^{K_{D}}\left(\frac{w}{u}\right)=1 .
$$

Suppose without loss of generality that $u$ is contained in both bases. Dividing everything by $u$, we can assume $u=1$. By possibly switching $v$ and $w$ and changing their signs, we can put the triple uniquely in the form $\{1, \lambda, \lambda-1\}$ with $\lambda^{(1)}>0$. Let $(a, b, c)$ be 
as in Proposition 3.3 applied to $M_{\lambda}$. Since $\lambda^{(1)} \lambda^{(2)}<0$ and $\left(\lambda^{(1)}-1\right)\left(\lambda^{(2)}-1\right)<0$, we must actually have $\lambda^{(1)}>1$, and it follows that $a+b+c<0$. The other necessary properties of $a, b$, and $c$ follow from Proposition 3.3.

Now assume $w^{(1)}=0$. In this case, $K_{D}=\mathbb{Q} \oplus \mathbb{Q}$, and there are two ways two put the triple in the form, $\{1, \lambda, \lambda-1\}$, where $\lambda=(1, s)$ with $s<0$ : either divide $\{u, v, w\}$ by $u$, or divide by $v$ and swap the first two elements. With $(a, b, c)$ as in Proposition 3.3 applied to $M_{\lambda}$, it follows that $a+b+c=0$ and $c<0$. The other necessary properties are clear, and the two $\lambda$ which arise in this way are $u / v$ and $v / u$, so they are reciprocal.

Now assume $w^{(2)}=0$. Dividing the triple by $u$ and switching $v$ and $w$ puts it in the form $\{1, \lambda, \lambda-1\}$ with $\lambda=(s, 0)$. Since $\lambda-1=(s-1,-1)$ has negative norm, we must have $s>1$. It follows that if $(a, b, c)$ are as in Proposition 3.3 applied to $M_{\lambda}$, we must have $c=0$ and $a+b<0$. Alternatively, we could have divided the triple by $v$ and rearranged the elements to put it in the form $\{1, \mu, \mu-1\}$ with $\mu=(t, 0)$. Then $t>1$ by the same argument, and $t=s /(s-1)$.

The converse statement that triples of the given form are admissible is not hard and will be left to the reader.

Just as for admissible pairs, we can define a map from the set of $Y_{D}$ prototypes to the set of isomorphism classes of quasi-invertible $\mathcal{O}_{D}$-modules with admissible triples, sending $(a, b, c, \bar{q})$ to $\left(M_{\lambda},\{1, \lambda, \lambda-1\}\right)$ as above. Since we identified two terminal or degenerate prototypes if they are related by the involutions (3-1) and (3-2), two prototypes have the same image if and only if they have the same $a, b$, and $c$.

\section{Abelian differentials}

In this section, we recall known material about Abelian differentials (holomorphic one-forms) on compact Riemann surfaces. In Section 4.1, we discuss the flat geometry associated to an Abelian differential. In Section 4.2, we discuss moduli spaces of Abelian differentials and the action of $\mathrm{SL}_{2} \mathbb{R}$ on these spaces. In Section 4.3, we discuss McMullen's results on the dynamics of this action in genus two.

\subsection{Flat geometry of Abelian differentials}

A Riemann surface with a nonzero Abelian differential carries a canonical flat geometry, which is closely related to the study of billiards in rational angled polygons, as well as the study of flows on moduli space. In this paper, this geometry will be useful because it will allow us to deform Abelian differentials using some concrete cut-and-paste operations which we will describe in this section. 
Translation surfaces Let $X$ be a Riemann surface with a nonzero Abelian differential $\omega$, and let $Z(\omega)$ be the discrete set of zeros of $\omega$. Every point of $X$ which is not a zero of $\omega$ has a neighborhood $U$ and a conformal map $\phi: U \rightarrow \mathbb{C}$ such that $\left.\omega\right|_{U}=\phi^{*}(d z)$. The conformal map $\phi$ can be defined explicitly by

$$
\phi(z)=\int_{z_{0}}^{z} \omega
$$

for a choice of base point $z_{0}$. These coordinates $\phi$ are unique up to translation by a constant.

We can also put $\omega$ in a standard form in the neighborhood of a zero or a pole at $z_{0}$. There is a neighborhood $U$ of $z_{0}$ and conformal map

$$
\phi_{0}:\left(U, z_{0}\right) \rightarrow(\mathbb{C}, 0)
$$

such that

$$
\text { (4-1) }\left.\quad \omega\right|_{U}= \begin{cases}\phi_{0}^{*}\left(z^{n} d z\right), & \text { if } z_{0} \text { is not a simple pole; } \\ \phi_{0}^{*}(a d z / z), & \text { if } z_{0} \text { is a simple pole with nonzero residue } a .\end{cases}
$$

Since the local coordinates $\phi$ away from the zeros of $\omega$ are unique up to translation by a constant, any translation invariant geometric structure on $\mathbb{C}$ is inherited by $X$. In particular, $X$ inherits a flat metric and an oriented foliation $\mathcal{F}_{h}$ coming from the foliation of the plane by horizontal lines. More generally, for slope $s \in \mathbb{P}^{1}(\mathbb{R})$, the surface $X$ has an orientable foliation $\mathcal{F}_{s}$ coming from the foliation of $\mathbb{C}$ by lines of slope $s$.

In terms of the Abelian differential $\omega$, the flat metric is just $|\omega|$. A vector $v$ is tangent to $\mathcal{F}_{h}$ in the positive direction if $\omega(v)>0$.

The flat metric has singularities at the zeros. From the coordinates (4-1), we see that a zero of order $n$ has a neighborhood isometric to a cone with cone angle $2 \pi(n+1)$, and the foliation $\mathcal{F}_{h}$ has $2 n+2$ leaves meeting at the zero. Zeros of orders one and two together with the foliation $\mathcal{F}_{h}$ are pictured in Figure 2.

To summarize, we have associated to an Abelian differential $(X, \omega)$, a flat metric on $X \backslash Z(\omega)$ together with a horizontal foliation $\mathcal{F}_{h}$ which is parallel with respect to a metric such that the points of $Z(\omega)$ are cone singularities of the metric. A surface with such a structure is sometimes called a translation surface. A translation surface is equivalent to a Riemann surface with a nonzero Abelian differential.

A geodesic on $(X, \omega)$ is called straight if it does not pass through any zeros of $\omega$. A straight geodesic which joins two zeros is called a saddle connection. Any straight, closed geodesic is contained in a cylinder on $X$ by taking nearby parallel geodesics. If 

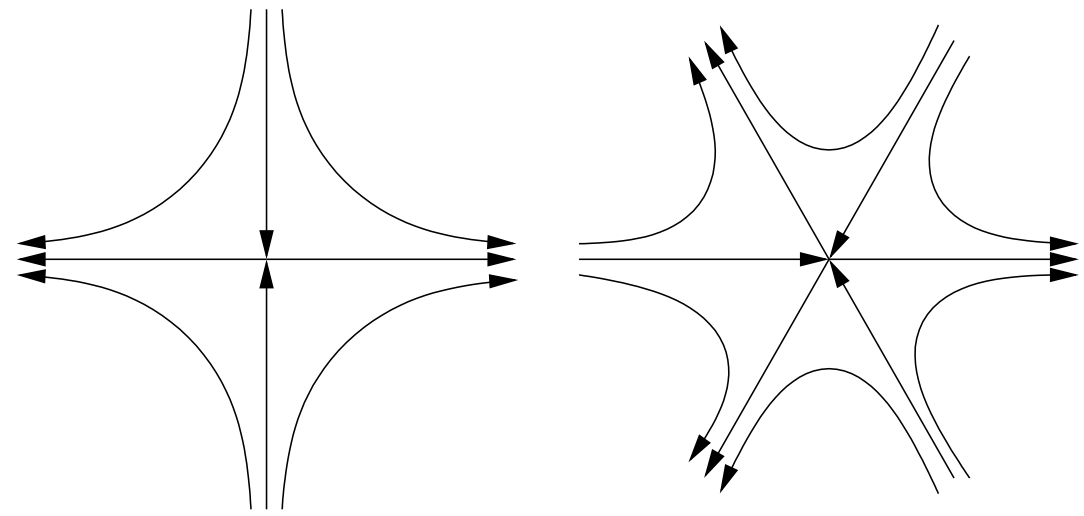

Figure 2: Zeros of order one and two

the genus of $X$ is greater than one, any cylinder can be extended until either end contains a zero of $\omega$ and each boundary component is a finite union of saddle connections. Such a cylinder is called a maximal cylinder.

Plumbing a cylinder Cylinders arise from simple poles: by (4-1), a simple pole of an Abelian differential has a neighborhood which is isometric to a half-infinite cylinder. Given a meromorphic Abelian differential $(X, \omega)$ with simple poles at $p$ and $q$ such that

$$
\operatorname{Res}_{p} \omega=-\operatorname{Res}_{q} \omega
$$

there is a simple surgery operation which allows us to replace the poles at $p$ and $q$ with a cylinders. Cut $X$ along two closed geodesics, one in each of the two half-infinite cylinders around $p$ and $q$, and then glue $X$ along the resulting boundary components by an isometry. The condition (4-2) means exactly that this gluing map is locally a translation; therefore, we get a new Abelian differential with two fewer poles. Call this operation plumbing a cylinder. It depends on two parameters: the height of the resulting cylinder and the amount of twisting of the gluing map.

We can reverse this operation to replace a cylinder with two simple poles. Just cut a cylinder along a closed geodesic, and then glue two half-infinite cylinders to the resulting boundary components Call this operation unplumbing a cylinder. These operations will be used in Section 6 to define polar coordinates around the boundary of the Deligne-Mumford compactification of moduli space.

Connected sums Two Abelian differentials $\left(X_{1}, \omega_{1}\right)$ and $\left(X_{2}, \omega_{2}\right)$ can be combined into one by taking a connected sum. This operation is studied in detail in [57]. 
Let $I$ be a line segment in the complex plane. Suppose $I$ is embedded in each of the surfaces $\left(X_{i}, \omega_{i}\right)$ by an isometric embedding $\epsilon_{i}: I \rightarrow X_{i}$ preserving the slope of $I$ as well as its length. Now cut each of the $X_{i}$ along $I$, and glue the surfaces together by gluing one boundary component along $I$ on one of the surfaces to the opposite boundary component on the other surface. The gluing maps are just translations in the flat structures on the $\left(X_{i}, \omega_{i}\right)$, so the resulting surface has a flat structure as well away from the ends of $I$. By the Riemann removable singularity theorem, the conformal structure and Abelian differential can actually be extended to the ends of $I$. Thus we obtain a new Abelian differential $(X, \omega)$. If the segments $\epsilon_{i}(I) \subset X_{i}$ are disjoint from the zeros of $\omega$, then the $\omega$ has a simple zero at each endpoint of the glued segment.

We call the resulting Abelian differential,

$$
(X, \omega)=\left(X_{1}, \omega_{1}\right) \#_{I}\left(X_{2}, \omega_{2}\right),
$$

the connected sum of $\left(X_{1}, \omega_{1}\right)$ and $\left(X_{2}, \omega_{2}\right)$ along $I$.

This construction can be modified in the obvious way to perform a self connected sum of an Abelian differential with itself, given two parallel embeddings of $I$ in that Abelian differential.

An Abelian differential $(X, \omega)$ resulting from a connected sum has two simple zeros $p$ and $q$ and two oriented saddle connections $I_{1}$ and $I_{2}$ going from $p$ to $q$ such that

$$
\int_{I_{1}} \omega=\int_{I_{2}} \omega
$$

Given any Abelian differential $(X, \omega)$ with a pair of oriented, embedded saddle connections $I_{1}$ and $I_{2}$ both beginning and ending at the same zeros and satisfying (4-3), we can reverse the connected sum operation. To do this, cut $X$ along $I_{1}$ and $I_{2}$ and then reglue to get a new Abelian differential $\left(X^{\prime}, \omega^{\prime}\right)$. Equation (4-3) implies that this gluing can be done by a translation. This operation is called splitting along $I_{1}$ and $I_{2}$ and is inverse to the connected sum operation.

In this paper, there are two main cases where we will use these constructions. First, suppose $\left(X_{1}, \omega_{1}\right)$ and $\left(X_{2}, \omega_{2}\right)$ are both genus one, and a segment $I \subset \mathbb{C}$ is embedded in each by embeddings $\epsilon_{i}$ as above. Then we can form the connected sum along $I$, and the resulting Abelian differential has genus two with two simple zeros. Conversely, splitting a genus two Abelian differential along a pair of saddle connections $I_{1}$ and $I_{2}$ such that $I_{1} \cup I_{2}$ separates the surface yields a pair of genus one Abelian differentials.

Second, given an genus one Abelian differential with two embedded segments $I_{i}$ which are parallel and of the same length, we can form a self connected sum along these segments. This again yields a genus two Abelian differential with two simple 
zeros. Conversely, splitting along a pair of saddle connections on a genus two Abelian differential satisfying (4-3) which don't separate the surface yields a single genus one Abelian differential.

Splitting a double zero There is also a cut-and-paste operation which replaces a zero of an Abelian differential with two zeros of lower order. This operation is explained in detail in Kontsevich and Zorich [43] and Eskin, Masur and Zorich [23]. We will describe this operation in the case of a double zero, the only case we need.

Let $(X, \omega)$ be an Abelian differential with a double zero at $p$, and choose a straight geodesic segment $I$ starting at $p$. The segment $I$ is a leaf of the foliation $\mathcal{F}_{\theta}$ of some slope $\theta$. On $X$, draw an " $X$ " composed of the segment $I$ and three other geodesic segments of the same length and slope as in Figure 3. (In this figure, leaves of $\mathcal{F}_{\theta}$ are represented by dotted or solid lines, with the orientation indicated by an arrow, and the " $\mathrm{X}$ " is represented by solid lines. Two consecutive segments meeting at the zero form a $180^{\circ}$ angle.) Assume that $I$ was chosen to be short enough so that each arm of the "X" is an embedded straight geodesic on $X$ not meeting any of the other arms.

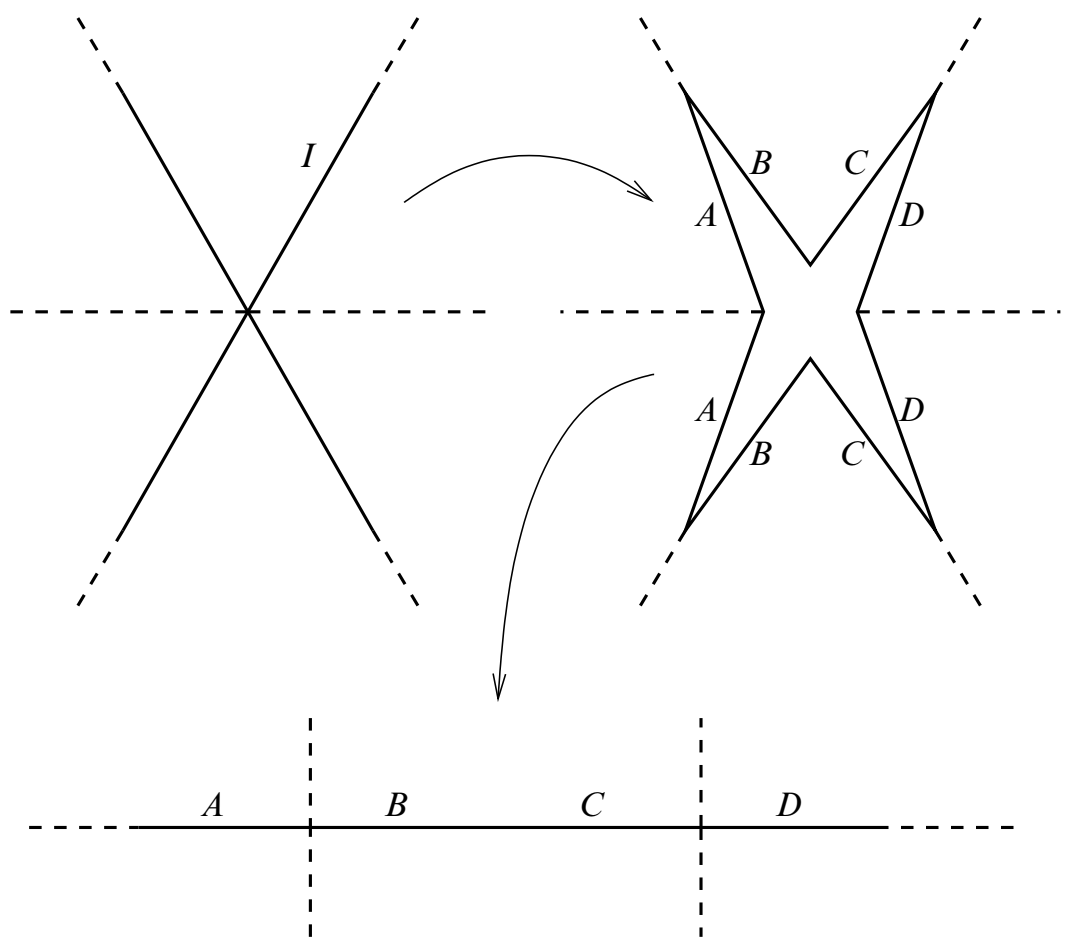

Figure 3: Splitting a double zero 
Now cut the surface along the " $\mathrm{X}$ " to obtain a surface with eight geodesic boundary components, and then glue the boundary components pairwise so that the components marked with the same letter in Figure 3 are glued together. The result is a new Abelian differential with simple zeros at the two points where four leaves of $\mathcal{F}_{\theta}$ meet in the figure. We will denote this new Abelian differential by

$$
(X, \omega) \#_{I} .
$$

This operation can also be reversed in the obvious way. Given a saddle connection of length $l$ joining two distinct zeros, draw two more segments of length $l / 2$ emanating from the zeros with the same slopes as in the bottom of Figure 3. If these segments can be drawn without intersecting each other or any other zero, then the process of splitting a double zero can be reversed: cut along these three segments and then reglue, following Figure 3 in reverse. This operation is called collapsing a saddle connection.

This operation, together with the connected sum operation, will be used in Section 12 to parameterize tubular neighborhoods of $\bar{W}_{D}$ and $\bar{P}_{D}$ in $Y_{D}$.

\subsection{Moduli of Abelian differentials}

In this subsection, we introduce the moduli space of Abelian differentials and discuss its geometry.

Teichmüller space Let $\Sigma_{g}$ be a connected, closed, oriented, topological surface of genus $g$, and let $\Sigma_{g, n}$ be a genus $g$ topological surface with $n$ marked points. A marked Riemann surface is a Riemann surface $X$ together with a homeomorphism $\Sigma_{g, n} \rightarrow X$. Two marked Riemann $(f, X)$ and $(g, Y)$ marked by $\Sigma_{g, n}$ are considered to be equivalent if $g \circ f^{-1}$ is homotopic to a conformal isomorphism by a homotopy fixing the marked points.

Let the Teichmüller space $\mathcal{T}\left(\Sigma_{g, n}\right)$ be the space of all Riemann surfaces marked by $\Sigma_{g, n}$ up to equivalence. It has a topology induced by the well-known Teichmüller metric and is homeomorphic to $\mathbb{C}^{N}$, where

$$
N= \begin{cases}1 & \text { if } g=1 \text { and } n=0 \text { or } 1 ; \\ n-1 & \text { if } g=1 \text { and } n>1 ; \\ n-3 & \text { if } g=0 \text { and } n>2 ; \\ 3 g-3+n & \text { if } g>1\end{cases}
$$

We will use the abbreviation $\mathcal{T}_{g, n}$ or $\mathcal{T}_{g}$ when we don't need to emphasize the surface $\Sigma_{g}$.

Bers gave $\mathcal{T}_{g, n}$ a complex structure by defining an embedding $B: \mathcal{T}_{g, n} \rightarrow \mathbb{C}^{N}$ which is a homeomorphism onto its image, a bounded domain in $\mathbb{C}^{N}$. 
Moduli space The modular group $\operatorname{Mod}\left(\Sigma_{g}\right)$ is the group of all self homeomorphisms of $\Sigma_{g}$ up to isotopy. Similarly, $\operatorname{Mod}\left(\Sigma_{g, n}\right)$ is the group of all self homeomorphisms which preserve the marked points, up to isotopy preserving the marked points.

An element $\gamma \in \operatorname{Mod}\left(\Sigma_{g, n}\right)$ acts on $\mathcal{T}\left(\Sigma_{g, n}\right)$ by replacing a marking

$$
f: \Sigma_{g, n} \rightarrow X
$$

with $f \circ \gamma^{-1}$. This defines a biholomorphic action of $\operatorname{Mod}\left(\Sigma_{g, n}\right)$ on $\mathcal{T}\left(\Sigma_{g, n}\right)$ which is properly discontinuous. Let $Z \subset \Sigma_{g, n}$ be the set of marked points. The stabilizer of a point $(f, X)$ is isomorphic to the group $\operatorname{Aut}(X, f(Z))$ of conformal automorphisms of $X$ preserving the marked points. In genus two, every Riemann surface has an order-two automorphism $J$, the hyperelliptic involution. This yields an element of order two in $\operatorname{Mod}\left(\Sigma_{2}\right)$ which acts trivially on $\mathcal{T}\left(\Sigma_{2}\right)$.

The moduli space of genus $g$ Riemann surfaces with $n$ marked points is the quotient

$$
\mathcal{M}_{g, n}=\mathcal{T}\left(\Sigma_{g, n}\right) / \operatorname{Mod}\left(\Sigma_{g, n}\right) .
$$

$\mathcal{M}_{g, n}$ is a complex orbifold.

Bundles of Abelian differentials Given a Riemann surface $X$, let $\Omega(X)$ be the space of Abelian differentials on $X$, a rank $g$ complex vector space. Let $\Omega \mathcal{T}_{g}$ be the space of all pairs $(X, \omega)$ with $X \in \mathcal{T}_{g}$ and $\omega \in \Omega(X)$ a nonzero Abelian differential. We can give $\Omega \mathcal{T}_{g}$ the structure of a trivial holomorphic punctured vector bundle as follows.

Over $\mathcal{T}_{g}$, there is the universal curve $\mathcal{C} \mathcal{T}_{g}$, defined by Bers in [10]. It is a complex manifold and comes with a proper map $\pi: \mathcal{C} \mathcal{T}_{g} \rightarrow \mathcal{T}_{g}$ whose fiber over a Riemann surface $X$ is isomorphic to $X$ itself. The cotangent bundle to the fibers of $\pi$ is a line bundle $\mathcal{L} \rightarrow \mathcal{C} \mathcal{T}_{g}$. The push-forward $\pi_{*} \mathcal{O}(\mathcal{L})$ of the sheaf of sections of $\mathcal{L}$ is a sheaf on $\mathcal{T}_{g}$. The following theorem follows from Bers [9].

Theorem 4.1 The sheaf $\pi_{*} \mathcal{O}(\mathcal{L})$ is the sheaf of sections of a trivial bundle over $\mathcal{T}_{g}$ whose fiber over a Riemann surface $X$ is $\Omega(X)$.

The action of the mapping class group $\operatorname{Mod}_{g}$ on $\mathcal{T}_{g}$ extends to an action on $\Omega \mathcal{T}_{g}$. The quotient is a rank $g$ orbifold vector bundle $\Omega \mathcal{M}_{g}$ over $\mathcal{M}_{g}$ whose fiber over a Riemann surface $X$ is the quotient $\Omega(X) / \operatorname{Aut}(X)$. This bundle is sometimes called the Hodge bundle.

In general, when $S$ is any sort of space of Riemann surfaces, $\Omega S$ will denote the natural bundle of Abelian differentials over $S$. 
Strata The bundles $\Omega \mathcal{T}_{g}$ have a natural stratification in terms of the types of zeros of the Abelian differentials. Each nonzero Abelian differential on a nonsingular Riemann surface has $2 g-2$ zeros, counting multiplicity. Given a sequence of integers $\mathbf{n}=\left(n_{i}\right)_{i=1}^{r}$ such that $\sum n_{i}=2 g-2$, let $\Omega \mathcal{T}_{g}(\mathbf{n})$ be the locus of all Abelian differentials which have $r$ zeros whose multiplicities are given by the $n_{i}$. This locus is a locally closed subset of $\Omega \mathcal{T}_{g}$, and by Veech [78] it is actually a complex submanifold. The quotient

$$
\Omega \mathcal{M}_{g}(\mathbf{n})=\Omega \mathcal{T}_{g}(\mathbf{n}) / \operatorname{Mod}
$$

is then a complex suborbifold of $\Omega \mathcal{M}_{g}$.

Period coordinates These strata have natural coordinates defined in terms of their periods by Veech [78] and Masur [49] which give the stratum $\Omega \mathcal{T}_{g}(\mathbf{n})$ the structure of an affine manifold.

There is a fiber bundle of homology groups

$$
\mathcal{H}_{1} \rightarrow \Omega \mathcal{T}_{g}(\mathbf{n})
$$

whose fiber over an Abelian differential $(X, \omega)$ is $H_{1}(X, Z(\omega)$; $\mathbb{Z})$, where $Z(\omega)$ is the set of zeros of $\omega$. Given two Abelian differentials $\left(X_{1}, \omega_{1}\right)$, and $\left(X_{2}, \omega_{2}\right) \in \Omega \mathcal{T}_{g}(\mathbf{n})$ with $\left(X_{2}, \omega_{2}\right)$ sufficiently close to $\left(X_{1}, \omega_{1}\right)$ there is a natural isomorphism

$$
H_{1}\left(X_{2}, Z\left(\omega_{2}\right)\right) \rightarrow H_{1}\left(X_{1}, Z\left(\omega_{1}\right)\right) .
$$

This defines a flat connection on $\mathcal{H}_{1}$, the Gauss-Manin connection.

Consider $(X, \omega) \in \Omega \mathcal{T}_{g}(\mathbf{n})$ and a small neighborhood $U$ of $(X, \omega)$. Any $\left(X^{\prime}, \omega^{\prime}\right) \in U$ defines an element of $H^{1}\left(X^{\prime}, Z\left(\omega^{\prime}\right) ; \mathbb{C}\right)$ via the periods of $\omega^{\prime}$. Composing this with the isomorphism

$$
H^{1}\left(X^{\prime}, Z\left(\omega^{\prime}\right) ; \mathbb{C}\right) \rightarrow H^{1}(X, Z(\omega) ; \mathbb{C})
$$

from the Gauss-Manin connection, we get a map

$$
\phi: U \rightarrow H^{1}(X, Z(\omega) ; \mathbb{C}),
$$

the period coordinates. Veech [78] showed that these are in fact biholomorphic coordinate charts.

A choice of basis of $H_{1}(X, Z(\omega) ; \mathbb{Z})$ defines an isomorphism

$$
H^{1}(X, Z(\omega) ; \mathbb{C}) \rightarrow \mathbb{C}^{2 g+n-1},
$$

where $n$ is the number of zeros of $\omega$. We can suppose that the basis is of the form,

$$
\left\{u_{1}, \ldots, u_{2 g}, v_{1}, \ldots, v_{n-1}\right\},
$$


where $\left\{u_{1}, \ldots, u_{2 g}\right\}$ is a symplectic basis of $H_{1}(X ; \mathbb{Z})$. Two such bases are related by a matrix in the group

$$
G=\left\{\left(\begin{array}{cc}
A & B \\
0 & C
\end{array}\right): A \in \mathrm{Sp}_{2 g} \mathbb{Z}, \quad B \in M_{2 g, n}(\mathbb{Z}), \quad C \in \mathrm{GL}_{n} \mathbb{Z}\right\} .
$$

Changing the basis by a matrix in $G$ changes the period coordinates by the transpose of this matrix. This gives $\Omega \mathcal{T}_{g}(\mathbf{n})$ the structure of an $\left(G, \mathbb{C}^{2 g+n-1}\right)$-manifold, and $\Omega \mathcal{M}_{g}(\mathbf{n})$ inherits the structure of a $\left(G, \mathbb{C}^{2 g+n-1}\right)$-orbifold.

Measures Since the action of $\mathrm{GL}_{n} \mathbb{Z}$ on $\mathbb{C}^{n}$ preserves Lebesgue measure, we can pull back this measure by the period coordinates to get the period measure $\mu(\mathbf{n})$ on $\Omega \mathcal{M}_{g}(\mathbf{n})$.

The flat metric defined by an Abelian differential has area given by

$$
\operatorname{Area}(\omega)=\frac{i}{2} \int_{X} \omega \wedge \bar{\omega}
$$

Let $\Omega_{\leq 1} \mathcal{M}_{g}(\mathbf{n})$ be the locus of Abelian differentials with $\operatorname{Area}(\omega) \leq 1$, and $\Omega_{1} \mathcal{M}_{g}(\mathbf{n})$ the locus of Abelian differentials with $\operatorname{Area}(\omega)=1$ (we will use the prefixes $\Omega_{\leq 1}$ and $\Omega_{1}$ to denote the analogous subsets of any space of Abelian differentials). We can define a measure $\mu^{\prime}(\mathbf{n})$ on $\Omega_{1} \mathcal{M}_{g}(\mathbf{n})$ by restricting $\mu(\mathbf{n})$ to $\Omega_{\leq 1} \mathcal{M}_{g}(\mathbf{n})$ and then projecting by the natural map $\Omega_{\leq 1} \mathcal{M}_{g}(\mathbf{n}) \rightarrow \Omega_{1} \mathcal{M}_{g}(\mathbf{n})$.

The following theorem was proved by Veech and Masur:

Theorem $4.2 \quad[49 ; 78]$ The measures $\mu^{\prime}(\mathbf{n})$ have finite total volume.

Action of $\mathbf{S L}_{\mathbf{2}} \mathbb{R}$ Given a nonzero Abelian differential $(X, \omega) \in \Omega \mathcal{T}_{g}$, we can as in Section 4.1 choose an atlas of coordinate charts $\phi_{i}: U_{i} \rightarrow \mathbb{C}$, which cover the complement in $X$ of the zeros of $\omega$, such that $\phi_{i}$ pulls back the form $d z$ on $\mathbb{C}$ to the form $\omega$ on $X$. These coordinate charts differ by translations on their overlap. Now, given an element $A \in \mathrm{SL}_{2} \mathbb{R}$, define new coordinate charts $\phi_{i}^{\prime}=A \circ \phi_{i}$ by composing with the usual action of $A$ on the complex plane. The new coordinates still differ by translations and so define a new translation surface. This defines a new Abelian differential $A \cdot(X, \omega) \in \Omega \mathcal{T}_{g}$ which has a different complex structure than $X$ unless $A$ happens to be a rotation in $\mathrm{SO}_{2} \mathbb{R}$. If $\left(X^{\prime}, \omega^{\prime}\right)=A \cdot(X, \omega)$, then there is a natural real-affine map $h_{A}:(X, \omega) \rightarrow\left(X^{\prime}, \omega^{\prime}\right)$ which takes zeros to zeros. The periods satisfy the relation,

$$
\omega^{\prime}\left(\left(h_{A}\right)_{*}(\gamma)\right)=A \cdot \omega(\gamma)
$$

for any $\gamma \in H_{1}(X, Z(\omega))$ with $Z(\omega)$ the set of zeros of $\omega$. 
This construction defines an action of $\mathrm{SL}_{2} \mathbb{R}$ on $\Omega \mathcal{T}_{g}$. This action commutes with the action of Mod and so defines an action of $\mathrm{SL}_{2} \mathbb{R}$ on $\Omega \mathcal{M}_{g}$ which preserves the locus $\Omega_{1} \mathcal{M}_{g}$ of Abelian differentials with area one.

Equation (4-5) can be used to show that the measure $\mu(\mathbf{n})$ is $\mathrm{SL}_{2} \mathbb{R}$ invariant, as is $\mu^{\prime}(\mathbf{n})$.

This action is closely related to the Teichmüller geodesic flow: the projection $\Omega \mathcal{M}_{g} \rightarrow$ $\mathcal{M}_{g}$ sends $\mathrm{SL}_{2} \mathbb{R}$ orbits to copies of the hyperbolic plane in $\mathcal{M}_{g}$ which are isometrically embedded with respect to the Teichmüller metric, and the restriction of the action to the one parameter subgroup of diagonal matrices is the Teichmüller geodesic flow.

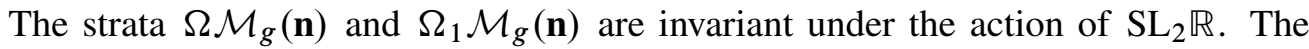
measures $\mu$ and $\mu^{\prime}$ defined in Section 5.2 are invariant measures by (4-5).

The natural projection $\Omega_{1} \mathcal{M}_{g} \rightarrow \mathbb{P} \Omega \mathcal{M}_{g}$ sends $\mathrm{SL}_{2} \mathbb{R}$ orbits to immersed copies of the hyperbolic plane, giving a foliation $\mathcal{F} \mathcal{M}_{g}$ of $\mathbb{P} \Omega \mathcal{M}_{g}$ by immersed hyperbolic planes. It is possible for a leaf of $\mathcal{F} \mathcal{M}_{g}$ to be a closed subset of $\mathbb{P} \Omega \mathcal{M}_{g}$. In that case it is called a Teichmüller curve because it is an algebraic curve whose projection to $\mathcal{M}_{g}$ is isometrically immersed with respect to the Teichmüller metric. It is an important unsolved problem to classify Teichmüller curves in $\mathbb{P} \Omega \mathcal{M}_{g}$ or more generally to classify orbit closures or invariant measures.

\section{3 $\mathrm{SL}_{2} \mathbb{R}$ orbits in genus two}

In genus two, McMullen has completely classified the orbit closures for the action of $\mathrm{SL}_{2} \mathbb{R}$ on $\Omega_{1} \mathcal{M}_{2}$ and the ergodic, invariant measures in the series of papers [52;57; 54; 56]. In this subsection, we will discuss the subsets of $\Omega_{1} \mathcal{M}_{2}$ which appear in this classification and which will be the focus of this paper.

A $\mathrm{SL}_{2} \mathbb{R}$-invariant subset $S$ of $\Omega_{1} \mathcal{M}_{2}$ corresponds to a subset $\mathbb{P} S$ of $\mathbb{P} \Omega \mathcal{M}_{2}$ which is saturated in the sense that leaves of the foliation $\mathcal{F} \mathcal{M}_{2}$ are either contained in or disjoint from $\mathbb{P} S$. Invariant measures on $\Omega_{1} \mathcal{M}_{2}$ correspond by a disintegration construction to holonomy invariant measures transverse to $\mathcal{F} \mathcal{M}_{2}$. In this paper, we will adopt this point of view and focus on leaves of this foliation rather than on $\mathrm{SL}_{2} \mathbb{R}$ orbits in $\Omega_{1} \mathcal{M}_{2}$.

Eigenform loci A form $(X,[\omega]) \in \mathbb{P} \Omega \mathcal{M}_{2}$ is an eigenform for real multiplication by $\mathcal{O}_{D}$ if the Jacobian of $X$ has real multiplication by $\mathcal{O}_{D}$ with $\omega$ a nonzero eigenform. Let the eigenform locus $E_{D}$ be the locus of all such pairs. The following proposition follows from [57, Corollary 5.7].

Proposition 4.3 $E_{D}$ is a closed, saturated subset of $\mathbb{P} \Omega \mathcal{M}_{2}$. 
An embedding $\iota_{i}: \mathcal{O}_{D} \rightarrow \mathbb{R}$ determines a map

$$
j_{i}: E_{D} \rightarrow X_{D}
$$

by sending an eigenform $(X, \omega) \in E_{D}$ to the pair $(\operatorname{Jac}(X), \rho)$, where

$$
\rho: \mathcal{O}_{D} \rightarrow \operatorname{End} \operatorname{Jac}(X)
$$

is chosen so that

$$
\omega(\rho(\lambda) \cdot \gamma)=\lambda^{(i)} \omega(\gamma)
$$

for each $\gamma \in H_{1}(X ; \mathbb{Z})$. Recall that in Section 2, we introduced the locus $P_{D}$ of Abelian varieties in $X_{D}$ which are polarized products of elliptic curves. The following follows from Proposition 5.4.

Proposition 4.4 The map $j_{i}$ is an isomorphism of $E_{D}$ onto $X_{D} \backslash P_{D}$.

In this paper, we will implicitly identify $E_{D}$ with $X_{D} \backslash P_{D}$ by the isomorphism $j_{1}$.

Let $\mathcal{F}_{D}$ be the foliation of $E_{D}$ by Riemann surfaces induced by the foliation $\mathcal{F M}_{2}$ of $\mathbb{P} \Omega \mathcal{M}_{2}$. The foliation $\mathcal{F}_{D}$ of $E_{D}$ extends to a foliation - which we will continue to call $\mathcal{F}_{D}$ - of $X_{D}$ defined by adding the connected components of $P_{D}$ as leaves of $\mathcal{F}_{D}$. McMullen [58] proved that this is actually a foliation of $X_{D}$.

Choice of real multiplication Given an eigenform $(X,[\omega]) \in E_{D}$, there are two choices of real multiplication $\rho: \mathcal{O}_{D} \rightarrow \operatorname{Jac}(X)$. We always choose $\rho$ so that $\omega$ is a 1 -eigenform in the terminology of Section 2.2.

Elliptic differentials There is a useful alternative characterization of eigenforms $(X, \omega)$ for real multiplication by $\mathcal{O}_{d^{2}}$.

A branched cover $f: X \rightarrow E$ from a Riemann surface to an elliptic curve is said to be primitive if it does not factor through an isogeny of elliptic curves $g$ : $E^{\prime} \rightarrow E$ of degree greater than one. Equivalently, $f$ is primitive if the map on homology $f_{*}: H_{1}(X ; \mathbb{Z}) \rightarrow H_{1}(E ; \mathbb{Z})$ is surjective.

An Abelian differential $(X, \omega)$ is called an elliptic differential if it is the pullback of a nonzero Abelian differential on an elliptic curve by a primitive branched cover. The degree of an elliptic differential is the degree of the cover.

Proposition 4.5 [57, Theorem 4.10] The locus of degree $d$ elliptic differentials in $\Omega \mathcal{M}_{2}$ is exactly $\Omega E_{d^{2}}$. 
There is a special class of elliptic differentials called square-tiled surfaces. A squaretiled surface is an Abelian differential which is pulled back from the square elliptic curve $(\mathbb{C} / \mathbb{Z}[i], d z)$ by some (not necessarily primitive) cover branched only over 0. Equivalently, an Abelian differential is square-tiled if and only if its absolute and relative periods all lie in the Gaussian integers $\mathbb{Z}[i]$. All square-tiled surfaces lie on Teichmüller curves.

Weierstrass curves A genus two Abelian differential is a Weierstrass form if it is an eigenform for real multiplication by some quadratic order $\mathcal{O}_{D}$ and if it has a double zero. Its discriminant is the discriminant $D$ of the order $\mathcal{O}_{D}$. The Weierstrass forms are parameterized by the Weierstrass form bundle $\Omega W_{D}$, which is a line bundle over the Weierstrass curve

$$
W_{D}:=\mathbb{P} \Omega W_{D} \subset X_{D}
$$

Theorem 4.6 [54] $W_{D}$ is a union of Teichmüller curves which is nonempty if and only if $D \geq 5$. If $D=9$ or if $D \neq \equiv 1 \bmod 8$, then $W_{D}$ is connected. Otherwise $W_{D}$ has two connected components.

Remark When $D=p^{2}$ for some prime $p$, this statement was first proved in [35].

When $W_{D}$ has two connected components, they are denoted by $W_{D}^{0}$ and $W_{D}^{1}$. They are distinguished by a topological invariant called the spin invariant. This is easy to describe when $D=d^{2}$. In that case, an Abelian differential $(X, \omega) \in \Omega W_{d^{2}}$ is branched over an elliptic curve $(E, v)$ by a $d$-fold branched cover $f: X \rightarrow E$. Let $p$ be the unique zero of $\omega$, one of the six Weierstrass points of $X$. Of the six Weierstrass points, $N$ of them have the same image in $E$ as $p$, with either $N=1$ or $N=3$. If $N=1$, then $(X, \omega)$ lies in $\Omega W_{D}^{0}$, and if $N=3$, then $(X, \omega)$ lies in $\Omega W_{D}^{1}$.

Let $\widetilde{W}_{D}$ be the inverse image of $W_{D}$ in the universal cover $\mathbb{W} \times \mathbb{M}$ of $X_{D}$.

Proposition 4.7 [52] $\widetilde{W}_{D}$ is a countable union of graphs of transcendental holomorphic maps $\mathbb{H} \rightarrow \mathbb{W}$.

Equivalently, $W_{D}$ is transverse to the absolute period foliation $\mathcal{A}_{D}$ of $X_{D}$ introduced in Section 2.3.

Period coordinates for $\boldsymbol{\Omega} \boldsymbol{E}_{\boldsymbol{D}}$ Let $E_{D}(1,1)=E_{D} \backslash W_{D}$, and let $\Omega E_{D}(1,1)$ be the bundle of nonzero eigenforms, that is, the line bundle over $E_{D}(1,1)$ whose fiber over $(X,[\omega])$ is the line spanned by $\omega$. We can define period coordinates on $\Omega E_{D}(1,1)$ in 
the same way as we defined period coordinates on the strata $\Omega \mathcal{M}_{g}(\mathbf{n})$. This material will only be used in Section 15.

Let

$$
\widetilde{E}_{D}(1,1)=\mathbb{H} \times \nVdash \backslash \widetilde{W}_{D},
$$

the inverse image of $E_{D}(1,1)$ in the universal cover of $X_{D}$, and let $\Omega \widetilde{E}_{D}(1,1)$ be the (trivial) bundle of eigenforms. Given an $(X, \omega) \in \Omega \widetilde{E}_{D}(1,1)$, the homology group $H_{1}(X, Z(\omega) ; \mathbb{Z})$ contains $H_{1}(X ; \mathbb{Z})$ as a subgroup which is equipped with an isomorphism

$$
\mathcal{O}_{D} \oplus \mathcal{O}_{D}^{\vee} \rightarrow H_{1}(X ; \mathbb{Z})
$$

The condition that $\omega$ is an eigenform is equivalent to the period map

$$
P_{\omega}: H_{1}(X ; \mathbb{Z}) \rightarrow \mathbb{C}
$$

being $\mathcal{O}_{D}$-linear, with $\mathcal{O}_{D}$ acting on $\mathbb{C}$ via the embedding $\iota_{1}$. Let

$$
H_{\mathcal{O}_{D}}^{1}(X, Z(\omega) ; \mathbb{C})
$$

be the subspace of $H^{1}(X, Z(\omega)$; $\mathbb{C})$ consisting of all linear maps

$$
H_{1}(X, Z(\omega) ; \mathbb{Z}) \rightarrow \mathbb{C}
$$

that are $\mathcal{O}_{D}$-linear on $H_{1}(X ; \mathbb{Z})$.

Now consider $(X, \omega) \in \Omega \widetilde{E}_{D}(1,1)$. For a sufficiently small neighborhood $U$ of $(X, \omega)$, any $\left(X^{\prime}, \omega^{\prime}\right) \in U$ defines an element of $H_{\mathcal{O}_{D}}^{1}(X, Z(\omega) ; \mathbb{C})$ by composing the period map $P_{\omega^{\prime}}$ with the isomorphism coming from the Gauss-Manin connection,

$$
H_{1}(X, Z(\omega) ; \mathbb{Z}) \rightarrow H_{1}\left(X^{\prime}, Z\left(\omega^{\prime}\right) ; \mathbb{Z}\right),
$$

which is $\mathcal{O}_{D}$-linear on $H_{1}(X ; \mathbb{Z})$. This give biholomorphic period coordinates

$$
\phi: U \rightarrow H_{\mathcal{O}_{D}}^{1}(X, Z(\omega) ; \mathbb{C}) .
$$

Consider a triple $(\alpha, \beta, \gamma) \subset H_{1}(X, Z(\omega) ; \mathbb{Z})$ such that:

- $(\alpha, \beta)$ is a basis of $H_{1}(X ; \mathbb{Z})$ over $\mathcal{O}_{D}$.

- $H_{1}(X ; \mathbb{Z}) \oplus\langle\gamma\rangle=H_{1}(X, Z(\omega) ; \mathbb{Z})$.

Such a triple determines an isomorphism

$$
H_{\mathcal{O}_{D}}^{1}(X, Z(\omega) ; \mathbb{Z}) \rightarrow \mathbb{C}^{3} .
$$

Two such triples are related by a matrix in the group

$$
G=\left\{\left(\begin{array}{cc}
A & B \\
0 & C
\end{array}\right) \in \mathrm{GL}_{3} K_{D}: A \in \operatorname{SL}\left(\mathcal{O}_{D} \oplus \mathcal{O}_{D}^{\vee}\right), B \in \mathcal{O}_{D} \oplus \mathcal{O}_{D}^{\vee}, C= \pm 1\right\}
$$


Changing the triple by a matrix in $G$ changes the period coordinates by the transpose of this matrix. This gives $\Omega \widetilde{E}_{D}(1,1)$ the structure of an $\left(G, \mathbb{C}^{3}\right)$-manifold, and $\Omega E_{D}(1,1)$ inherits the structure of a $\left(G, \mathbb{C}^{3}\right)$-orbifold.

These period coordinates are compatible with the action of $\mathrm{SL}_{2} \mathbb{R}$ in the sense that $\phi: U \rightarrow \mathbb{C}^{3}$ commutes with the two $\mathrm{SL}_{2} \mathbb{R}$ actions, where $\mathrm{SL}_{2} \mathbb{R}$ acts on $\mathbb{C}$ coordinatewise by identifying each $\mathbb{C}$ factor with $\mathbb{R}^{2}$.

By pulling back Lebesgue measure on $\mathbb{C}^{3}$ via these charts, we define a measure $\mu_{D}$ on $E_{D}(1,1)$. We can use this measure to define a measure $\mu_{D}^{\prime}$ on $\Omega_{1} E_{D}(1,1)$, using the same trick we used to define the measures $\mu^{\prime}(\mathbf{n})$ above Theorem 4.2.

Theorem 4.8 [57] The measures $\mu_{D}^{\prime}$ are finite, ergodic, $\mathrm{SL}_{2} \mathbb{R}$-invariant measures.

Cusps of $\boldsymbol{W}_{\boldsymbol{D}}$ We describe here McMullen's classification of cusps of $W_{\boldsymbol{D}}$ from [54], which will play a crucial role in our calculation of $\chi\left(W_{D}^{\epsilon}\right)$.

Given an Abelian differential $(X, \omega) \in \Omega_{1} \mathcal{M}_{g}$ and a slope $s \in \mathbb{P}^{1}(\mathbb{R})$, the foliation $\mathcal{F}_{S}$ of $X$ is periodic if every leaf of the foliation is either a saddle connection joining zeros of $\omega$, or a closed loop on $X$. A periodic foliation divides $X$ into finitely many maximal cylinders $C_{i}$ foliated by closed leaves of $\mathcal{F}_{s}$. The complement of $\bigcup C_{i}$ is a union of saddle connections on $X$ called the spine of $(X, \omega)$.

Two periodic foliations $\mathcal{F}_{s_{i}}$ of $\left(X_{i}, \omega_{i}\right) \in \Omega_{1} \mathcal{M}_{g}$ are equivalent if there is some $A \in \mathrm{SL}_{2} \mathbb{R}$ such that $A \cdot\left(X_{1}, \omega_{1}\right)=\left(X_{2}, \omega_{2}\right)$ and $A \cdot s_{1}=s_{2}$.

Now restrict to the case of Weierstrass forms $(X, \omega) \in W_{D}$. If $D$ is not square, then a periodic foliation decomposes $X$ into two cylinders; if $D$ is square, then a periodic foliation decomposes $X$ into either one or two cylinders (see Theorem 4.3 of [54]).

A Weierstrass form $(X, \omega) \in \Omega_{1} W_{D}$ together with a periodic foliation $\mathcal{F}_{S}$ determines a cusp of $W_{D}$. Let $N \subset \mathrm{SL}_{2} \mathbb{R}$ be the upper-triangular subgroup consisting of all matrices

$$
N_{t}=\left(\begin{array}{ll}
1 & t \\
0 & 1
\end{array}\right) \text {. }
$$

Let $g \in \mathrm{SL}_{2} \mathbb{R}$ be some matrix taking $s$ to 0 (so that the horizontal foliation of $g \cdot(X, \omega)$ is periodic). The map

$$
t \mapsto g^{-1} N_{t} g
$$

defines a path on $\Omega_{1} W_{D}$, which happens to cover a closed horocycle $h$ on $W_{D}$. Replacing $(X, \omega, s)$ with an equivalent periodic foliation gives a horocycle homotopic to $h$. Homotopy classes of closed horocycles on a Riemann surfaces $S$ correspond to cusps of $S$, so this construction associates a cusp of $W_{D}$ to every equivalence class 
of periodic foliations. This correspondence is in fact a bijection; see, for example, Theorem 4.1 of [54].

We say that a cusp of $W_{D}$ is a one-cylinder cusp or a two-cylinder cusp if the associated periodic foliation has one cylinder or two cylinders respectively. One cylinder cusps only arise on $W_{D}$ if $D$ is square. An example of a foliation associated to a one-cylinder cusp is given by gluing the edges of a three-by-one rectangle as in Figure 4 and taking the horizontal foliation.

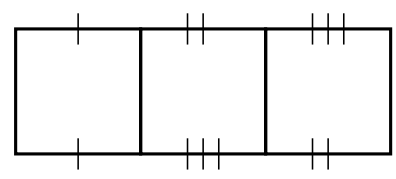

Figure 4: One cylinder cusp of $W_{9}$

McMullen [54] classified the cusps of $W_{D}$ by identifying them with splitting prototypes. We will describe his classification here using the equivalent $W_{D}$-prototypes from Section 3 .

To the prototype $P=(a, b, c, \bar{q})$, we associate the surface $\left(X_{P}, \omega_{P}\right)$ formed by gluing a square in $\mathbb{C}$ with unit length sides to the parallelogram with sides $0, \lambda=\lambda(P), r$, and $\lambda+r$, where $\lambda$ is the unique positive root of

$$
a \lambda^{2}+b \lambda+c=0
$$

and

$$
r=-\frac{q}{c} \lambda-i \frac{a}{c} \lambda,
$$

and then gluing opposite sides of the resulting polygon (see Figure 5). This $\left(X_{P}, \omega_{P}\right)$ is an eigenform for real multiplication by $\mathcal{O}_{D}$ where $D$ is the discriminant of $P$. The

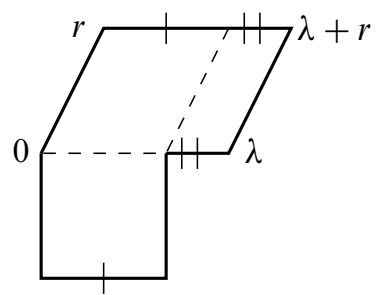

Figure 5: Cusp of $W_{D}$

horizontal foliation of $\left(X_{P}, \omega_{P}\right)$ is periodic and so determines a cusp $w_{P}$ of $W_{D}$. 
Theorem 4.9 [54, Theorem 4.1] The map $P \mapsto w_{P}$ described above determines a bijection between the set of $W_{D}$-prototypes and the set of two-cylinder cusps of $W_{D}$.

We will also need to know which connected component of $W_{D}$ contains a given cusp $w_{P}$. Given an order $\mathcal{O}_{D}$, define the conductor of $\mathcal{O}_{D}$ to be the positive integer $f$ such that $D=f^{2} E$ with $E$ a fundamental discriminant.

Theorem 4.10 $\left[54\right.$, Theorem 5.3] The cusp $w_{P}$ of $W_{D}$ associated to the $W_{D^{-}}$ prototype $P=(a, b, c, \bar{q})$ is contained in the component $W_{D}^{\epsilon(P)}$, where

$$
\epsilon(P) \equiv \frac{b-f}{2}+(a+1)(q+c+q c) \quad(\bmod 2),
$$

and $f$ is the conductor of $\mathcal{O}_{D}$.

Other Teichmüller curves When the discriminant $D$ is square, there is an infinite family of Teichmüller curves on $X_{d^{2}}$ parameterizing Abelian differentials with two simple zeros. Given $(X, \omega) \in \Omega X_{d^{2}}$, let $f:(X, \omega) \rightarrow(E, v)$ its associated degree $d$ torus cover. Define $\Omega W_{d^{2}}[n]$ to be the locus of all differentials such that the two branch points of $f$ in $E$ differ by torsion of degree exactly $n$ in the group law on $E$, and define $W_{d^{2}}[n] \subset X_{d^{2}}$ be its projectivization. The locus $W_{d^{2}}[n]$ is a union of Teichmüller curves. Note that $W_{d^{2}}[0]$ contains $W_{d^{2}}$ but is in general larger because $X$ can have distinct zeros which are branched over the same point of $E$.

There is one more example of a genus two Teichmüller curve which comes from an infinite family discovered by Veech. For even $n$, consider the Abelian differential obtained by gluing opposite sides of the regular $n$-gon. Veech [79] showed that the $\mathrm{SL}_{2} \mathbb{R}$ orbit of this differential lies on a Teichmüller curve $D_{n}$. The Abelian differential coming from the decagon is an genus two Abelian differential with two simple zeros. Its orbit is a Teichmüller $D_{10}$ curve lying on $X_{5}$.

Classification of $S_{2} \mathbb{R}$ orbit closures in genus two It happens that every $\mathrm{SL}_{2} \mathbb{R}$ orbit-closure on $\Omega_{1} \mathcal{M}_{2}$ or ergodic, invariant measure is one of the ones just described.

Theorem 4.11 $[57 ; 56]$ Every closure of an $\mathrm{SL}_{2} \mathbb{R}$ orbit in $\Omega_{1} \mathcal{M}_{2}$ is either all of $\Omega_{1} \mathcal{M}_{2}$, or one of the following submanifolds: $\Omega_{1} \mathcal{M}_{2}(2), \Omega_{1} E_{D}, \Omega_{1} D_{10}$, a connected component of $\Omega_{1} W_{D}$, or a component of $\Omega_{1} W_{d^{2}}[n]$.

Furthermore each of these orbit closure carries a unique ergodic, absolutely continuous, $\mathrm{SL}_{2} \mathbb{R}$-invariant probability measure. These are all of the ergodic, $\mathrm{SL}_{2} \mathbb{R}$-invariant probability measures on $\Omega_{1} \mathcal{M}_{2}$.

The only gap remaining in this classification is to describe the connected components of $W_{d^{2}}[n]$. 


\section{Deligne-Mumford compactification of moduli space}

\subsection{Stable Riemann surfaces}

A nodal Riemann surface is a connected, compact, one-dimensional, complex analytic space with only nodes as singularities (a node is a transverse crossing of two nonsingular branches). Equivalently, a nodal Riemann surface can be regarded as a finite type Riemann surface with finitely many cusps which have been identified pairwise to form nodes. A connected component of a nodal Riemann surface $X$ with its nodes removed is called a part of $X$, and the closure of a part of $X$ is an irreducible component of $X$. In this paper the genus of a nodal Riemann surface $X$ will mean its arithmetic genus,

$$
g=1-\chi\left(\mathcal{O}_{X}\right)
$$

where $\mathcal{O}_{X}$ is the structure sheaf of $X$. In topological terms, the arithmetic genus of a nodal Riemann surface $X$ is the genus of the nonsingular Riemann surface obtained by replacing each node of $X$ with an annulus.

A stable Riemann surface is a connected nodal Riemann surface for which each part has non-Abelian fundamental group (or equivalently negative Euler characteristic).

A nodal Riemann surface $X$ has a normalization $\tilde{X} \rightarrow X$ defined by separating the two branches passing through each node of $X$.

Stable Abelian differentials A stable Abelian differential on a stable Riemann surface $X$ is a holomorphic 1 -form on $X$ minus its nodes such that:

- Its restriction to each part of $X$ has at worst simple poles at the cusps.

- At two cusps which have been identified to form a node, the differential has opposite residues.

These properties can be conveniently rephrased using the normalization. If $\omega$ is a meromorphic Abelian differential on $X$, and $f: \tilde{X} \rightarrow X$ is the normalization, then $\omega$ is stable if and only if $f^{*} \omega$ has at worst simple poles, and for every $q \in X$,

$$
\sum_{f(p)=q} \operatorname{Res}_{p}\left(f^{*} \omega\right)=0
$$

A stable Riemann surface $X$ has a dualizing sheaf $\omega_{X}$. A stable Abelian differential on a stable Riemann surface is just a global section of $\omega_{X}$. This is discussed in Harris and Morrison [31] and Hartshorne [32]. 
The stable Abelian differentials on a genus $g$ singular Riemann surface $X$ form a complex vector space which we will write as $\Omega(X)$. The complex dimension of $\Omega(X)$ is $g$ :

$$
\begin{aligned}
h^{0}\left(X, \omega_{X}\right) & =h^{1}\left(X, \mathcal{O}_{X}\right) \\
& =1-\chi\left(\mathcal{O}_{X}\right) \\
& =g .
\end{aligned}
$$

This fact also follows easily from the Riemann-Roch Theorem. It is also proved in Serre [72, Theorem IV.2].

We will often use the term "Abelian differential" as shorthand for "Riemann surface together with an Abelian differential". We will call a node of a stable Abelian differential $(X, \omega)$ a polar node if $\omega$ has a pole there and a holomorphic node otherwise.

Jacobians The classical notion of the Jacobian variety of a nonsingular Riemann surface can be extended to singular Riemann surfaces.

If $X$ is a possibly singular Riemann surface, let $X_{0}$ be the set of nonsingular points of $X$. Then there is a natural map $H_{1}\left(X_{0} ; \mathbb{Z}\right) \rightarrow \Omega^{*}(X)$, given by integrating forms over homology classes. The Jacobian variety of $X$ is the quotient

$$
\operatorname{Jac}(X)=\Omega^{*}(X) / H_{1}\left(X_{0} ; \mathbb{Z}\right) .
$$

In the case of a stable Riemann surface $X$, it is easy to describe the kernel of the map $H_{1}\left(X_{0} ; \mathbb{Z}\right) \rightarrow \Omega^{*}(X)$. It is the subgroup of $H_{1}\left(X_{0} ; \mathbb{Z}\right)$ generated by the relations $\alpha-\beta$ when $\alpha$ and $\beta$ are homology classes generated by curves going around the same node of $X$ in the "same direction" on opposite sides as in Figure 6.

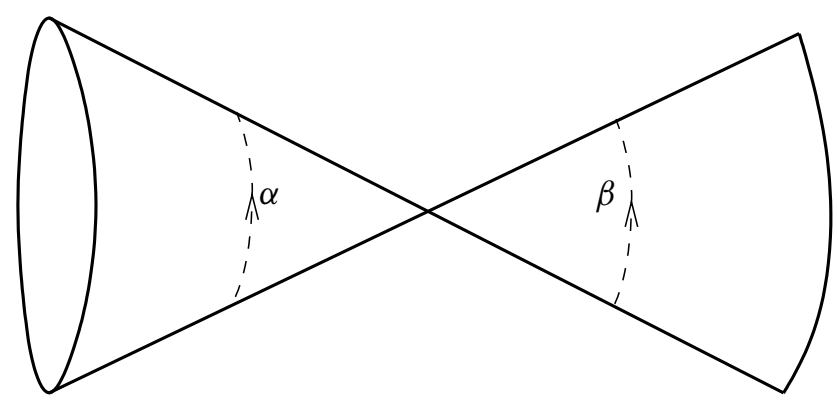

Figure 6: Curves around a node

There is an exact sequence which relates the Jacobian of a nodal Riemann surface to the Jacobian of its normalization. If $\tilde{X} \rightarrow X$ is the normalization of $X$, then holomorphic 
Abelian differentials on $\tilde{X}$ restrict to stable Abelian differentials on $X$, so there is a natural map $\operatorname{Jac}(X) \rightarrow \operatorname{Jac}(\tilde{X})$. We get an exact sequence:

$$
0 \rightarrow\left(\mathbb{C}^{*}\right)^{n} \rightarrow \operatorname{Jac}(X) \rightarrow \operatorname{Jac}(\tilde{X}) \rightarrow 0,
$$

where $n$ is the difference of the genera of $X$ and $\tilde{X}$. This realizes $\operatorname{Jac}(X)$ as a semi-Abelian variety.

For example, if $X$ has genus two and one separating node, then $\tilde{X}$ is the disjoint union of two elliptic curves. The Jacobian of $X$ is then the product of these two elliptic curves.

If $X$ has genus two and one nonseparating node, then the normalization of $X$ is an elliptic curve $E$. The Jacobian of $X$ is then an extension of $E$ by $\mathbb{C}^{*}$. The original stable Riemann surface $X$ can be recovered from $\operatorname{Jac}(X)$.

If $X$ has genus two and two (or three) nonseparating nodes, then $\tilde{X}$ is $\mathbb{P}^{1}$ (or $\mathbb{P}^{1} \cup \mathbb{P}^{1}$ respectively), so $\operatorname{Jac}(X) \cong \mathbb{C}^{*} \times \mathbb{C}^{*}$. This example shows that two distinct nodal Riemann surfaces may have the same Jacobian.

The Jacobian of $X$ can also be identified with $\operatorname{Pic}^{0}(X)$, the group of all line bundles on $X$ which have degree zero on each irreducible component of $X$ [31, p 250].

\subsection{Deligne-Mumford compactification}

Marked stable Riemann surfaces Given a stable surface $X$ of genus $g$, a collapse of $\Sigma_{g}$ onto $X$ is a continuous surjection $f: \Sigma_{g} \rightarrow X$ with the following properties:

- The inverse image of each node of $X$ is a Jordan curve on $\Sigma_{g}$.

- Each component of $\Sigma \backslash f^{-1}(N)$, where $N$ is the set of nodes of $X$, maps homeomorphically, preserving the orientation, onto a part of $X$.

If $X$ is nonsingular, a collapse is just a homeomorphism $\Sigma_{g} \rightarrow X$. These maps were introduced by Bers; in his terminology a collapse is called a strong deformation.

A marked stable Riemann surface is a stable Riemann surface $X$, together with a collapse $\Sigma_{g} \rightarrow X$. Two markings $f_{i}: \Sigma_{g} \rightarrow X_{i}$ are equivalent if there is a conformal isomorphism $g: X_{1} \rightarrow X_{2}$ such that the following diagram commutes up to homotopy.

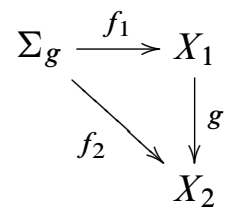

We will sometimes denote by $\left[f: \Sigma_{g} \rightarrow X\right]$ the class of all marked surfaces equivalent to $f: \Sigma_{g} \rightarrow X$. 
Augmented Teichmüller space The Teichmüller space $\mathcal{T}\left(\Sigma_{g}\right)$ is contained in the augmented Teichmüller space $\overline{\mathcal{T}}\left(\Sigma_{g}\right)$, the set of all marked stable genus $g$ Riemann surfaces up to equivalence. Let $\partial \mathcal{T}\left(\Sigma_{g}\right)=\overline{\mathcal{T}}\left(\Sigma_{g}\right) \backslash \mathcal{T}\left(\Sigma_{g}\right)$.

We give $\overline{\mathcal{T}}\left(\Sigma_{g}\right)$ a topology as follows. Given a closed curve $\gamma$ on $\Sigma_{g}$, define a function $l_{\gamma}: \overline{\mathcal{T}}\left(\Sigma_{g}\right) \rightarrow \mathbb{R} \cup\{\infty\}:$ if $\gamma$ is not homotopic to a curve on $X$ disjoint from the nodes, let $l_{\gamma}(X)=\infty$; if $\gamma$ is homotopic to a node of $X$, let $l_{\gamma}(X)=0$; otherwise let $l_{\gamma}(X)$ be the length of the unique geodesic homotopic to $\gamma$ in the Poincarè metric on $X$ minus its nodes. Give $\overline{\mathcal{T}}\left(\Sigma_{g}\right)$ the smallest topology such that $l_{\gamma}$ is continuous for every closed curve $\gamma$ on $\Sigma_{g}$. The induced subspace topology on $\mathcal{T}\left(\Sigma_{g}\right)$ agrees with that defined by the Teichmüller metric on $\mathcal{T}\left(\Sigma_{g}\right)$.

Abikoff [1] showed that this topology is equivalent to other natural topologies on $\overline{\mathcal{T}}\left(\Sigma_{g}\right)$, such as those obtained by looking at quasiconformal maps or quasi-isometries defined outside a neighborhood of the nodes.

Another useful topology which is equivalent to this one is the conformal topology which is defined as follows. Let $\left[f: \Sigma_{g} \rightarrow X\right] \in \partial \mathcal{T}\left(\Sigma_{g}\right)$, and let $U \subset X$ be a neighborhood of the nodes of $X$. Let $V_{U}$ be the set of all $\left[g: \Sigma_{g} \rightarrow Y\right] \in \overline{\mathcal{T}}\left(\Sigma_{g}\right)$ for which $f$ and $g$ can be adjusted by homotopies so that $\left.g \circ f^{-1}\right|_{X \backslash \bar{U}}$ is conformal. The set of all $V_{U}$ as $U$ runs over all neighborhoods of the nodes of $X$ define a neighborhood basis of $X$ in $\overline{\mathcal{T}}\left(\Sigma_{g}\right)$. Together with the open sets of $\mathcal{T}\left(\Sigma_{g}\right)$, this defines a topology on $\overline{\mathcal{T}}\left(\Sigma_{g}\right)$. It is well-known that this topology is equivalent to the one defined above; however, a proof does not exist in the literature.

A curve system on $\Sigma_{g}$ is a collection of simple closed curves on $\Sigma_{g}$, none of which are isotopic to any other or to a point. For each curve system $S$, there is a subspace $\mathcal{T}\left(\Sigma_{g}, S\right) \subset \partial \mathcal{T}\left(\Sigma_{g}\right)$ consisting of marked stable Riemann surfaces $\left[f: \Sigma_{g} \rightarrow X\right]$ topologically equivalent to the collapse $\Sigma_{g} \rightarrow \Sigma_{g} / S$ obtained by identifying each curve in $S$ to a point. For each connected component $\Sigma_{g}^{i}$ of $\Sigma_{g} \backslash S$, let $S_{i}$ be the closed surface with marked points obtained by collapsing the boundary components of $\Sigma_{g}^{i}$ to points and regarding the images of the boundary components as marked points. There is a natural isomorphism

$$
\mathcal{T}\left(\Sigma_{g}, S\right) \cong \prod_{i} \mathcal{T}\left(S_{i}\right)
$$

Deligne-Mumford compactification The action of $\operatorname{Mod}\left(\Sigma_{g}\right)$ of $\mathcal{T}\left(\Sigma_{g}\right)$ extends to an action on $\overline{\mathcal{T}}\left(\Sigma_{g}\right)$. The quotient,

$$
\overline{\mathcal{M}}_{g}=\overline{\mathcal{T}}\left(\Sigma_{g}\right) / \operatorname{Mod}\left(\Sigma_{g}\right),
$$


is the Deligne-Mumford compactification of $\mathcal{M}_{g}$. It is a compact orbifold whose points naturally parameterize stable Riemann surfaces of genus $g$.

Given a curve system $S \subset \Sigma_{g}$, let $\mathcal{M}_{g}(S) \subset \overline{\mathcal{M}}_{g}$ be the stratum of stable Riemann surfaces homeomorphic to the topological stable surface $\Sigma_{g} / S$. This is a locally closed subset of $\overline{\mathcal{M}}_{g}$, and $\overline{\mathcal{M}}_{g}$ is the disjoint union of all of the $\mathcal{M}_{g}(S)$ as $S$ ranges over all isotopy classes of curve systems on $\Sigma_{g}$ up to the action of the modular group.

Dehn space Define for any curve system $S$ on $\Sigma_{2}$,

$$
\overline{\mathcal{T}}\left(\Sigma_{2}, S\right)=\mathcal{T}\left(\Sigma_{2}\right) \cup \bigcup_{T \subset S} \mathcal{T}\left(\Sigma_{2}, T\right),
$$

where the union is over all curve systems $T \subset S$.

Given a curve system $S$ on $\Sigma_{g}$, let $\operatorname{Tw}(S)$ be the group generated by Dehn twists around the curves of $S$. It is an Abelian group isomorphic to $\mathbb{Z}^{h}$, where $h$ is the number of curves in $S$. Define the Dehn space $\mathcal{D}\left(\Sigma_{g}, S\right)$ to be

$$
\mathcal{D}\left(\Sigma_{g}, S\right)=\overline{\mathcal{T}}\left(\Sigma_{2}, S\right) / \operatorname{Tw}(S)
$$

where the union is over all sub-curve-systems of $S$. We will sometimes use the notation $\mathcal{D}_{g}(S)=\mathcal{D}\left(\Sigma_{g}, S\right)$ when we don't need to emphasize the surface $\Sigma_{g}$. Bers called $\mathcal{D}\left(\Sigma_{g}, S\right)$ the strong deformation space.

Let $\operatorname{Stab}(S)$ be the subgroup of $\operatorname{Mod}\left(\Sigma_{g}\right)$ which maps $S$ to an isotopic curve system. $\operatorname{Tw}(S)$ is a normal subgroup of $\operatorname{Stab}(S)$, and the quotient,

$$
\operatorname{Mod}\left(\Sigma_{g}, S\right)=\operatorname{Stab}(S) / \operatorname{Tw}(S)
$$

is the mapping class group of the topological stable surface obtained by collapsing each curve in $S$ to a point.

The group $\operatorname{Mod}\left(\Sigma_{g}, S\right)$ acts on $\mathcal{D}\left(\Sigma_{g}, S\right)$, and the natural map

$$
\pi: \mathcal{D}\left(\Sigma_{g}, S\right) \rightarrow \overline{\mathcal{M}}_{g}
$$

is equivariant with respect to this action. Bers showed:

Proposition 5.1 [11, p 1221] If $X \in \mathcal{D}\left(\Sigma_{g}, S\right)$, and $G \subset \operatorname{Mod}\left(\Sigma_{g}, S\right)$ is the stabilizer of $X$, then there is a neighborhood $U \subset \mathcal{D}\left(\Sigma_{g}, S\right)$ of $X$, stable under the action of $G$, such that $\pi$ factors through to a map, $\bar{\pi}: U / G \rightarrow \overline{\mathcal{M}}_{g}$, which is homeomorphic onto its image. 
Complex structure We now describe the complex structure on $\overline{\mathcal{M}}_{g}$, following Bers' approach [12].

In [8], Bers defined an embedding $B: \mathcal{T}\left(\Sigma_{g}\right) \rightarrow \mathbb{C}^{n}$, where

$$
n= \begin{cases}1 & \text { if } g=1 \\ 3 g-3 & \text { if } g>1\end{cases}
$$

It is a homeomorphism onto a bounded domain, and $\mathcal{T}\left(\Sigma_{g}\right)$ inherits the complex structure on $\mathbb{C}^{n}$. Ahlfors [2] showed that this is the unique complex structure on $\mathcal{T}\left(\Sigma_{g}\right)$ for which the periods of Abelian differentials vary holomorphically.

The modular group $\operatorname{Mod}\left(\Sigma_{g}\right)$ acts biholomorphically on $\mathcal{T}\left(\Sigma_{g}\right)$ with this complex structure, so $\mathcal{M}_{g}$ inherits the structure of a complex orbifold.

Bers gives $\overline{\mathcal{M}}_{g}$ a complex structure by first giving $\mathcal{D}\left(\Sigma_{g}, S\right)$ a complex structure for each curve system $S$. First define a sheaf of rings $\mathcal{O}$ on $\mathcal{D}\left(\Sigma_{g}, S\right)$ to be the sheaf consisting of all continuous functions $\mathcal{D}\left(\Sigma_{g}, S\right) \rightarrow \mathbb{C}$ which are holomorphic on

$$
\mathcal{D}_{0}\left(\Sigma_{g}, S\right)=\mathcal{T}\left(\Sigma_{g}\right) / \operatorname{Tw}(S) \subset \mathcal{D}\left(\Sigma_{g}, S\right)
$$

with respect to the complex structure on $\mathcal{D}_{0}\left(\Sigma_{g}, S\right)$ induced by the Bers embedding of Teichmüller space. Given a domain, $U \subset \mathcal{D}\left(\Sigma_{g}, S\right)$, we consider a function $f: U \rightarrow \mathbb{C}$ to be holomorphic if it is in $\mathcal{O}$. Bers showed that this defines an integrable complex structure on $\mathcal{D}\left(\Sigma_{g}, S\right)$ by giving a biholomorphic isomorphism of $\mathcal{D}\left(\Sigma_{g}, S\right)$ with a bounded domain in $\mathbb{C}^{n}$ parameterizing a certain family of Kleinian groups:

Proposition 5.2 [12] Let $S=\bigcup S_{i}$ be a curve system on $\Sigma_{g}$. There is a biholomorphic map

$$
B: \mathcal{D}\left(\Sigma_{g}, S\right) \rightarrow \mathbb{C}^{n}
$$

onto a bounded domain in $\mathbb{C}^{n}$. A curve $S_{i}$ is homotopic to a node on a marked stable Riemann surface $X \in \mathcal{D}\left(\Sigma_{g}, S\right)$ if and only if

$$
z_{i}(B(X))=0
$$

where the $z_{i}$ are the coordinates on $\mathbb{C}^{n}$.

Similar embeddings of $\mathcal{D}\left(\Sigma_{g}, S\right)$ are constructed in Marden [48] and Kra [44].

Using Proposition 5.1, we give $\overline{\mathcal{M}}_{g}$ the unique complex structure which makes all of the maps $\mathcal{D}\left(\Sigma_{g}, S\right) \rightarrow \overline{\mathcal{M}}_{g}$ holomorphic.

Baily showed in $[5 ; 6]$ that $\mathcal{M}_{g}$ also has the structure of a quasi-projective variety. Wolpert [82] showed using the Weil-Petersson metric on $\mathcal{M}_{g}$ that $\overline{\mathcal{M}}_{g}$ has the structure 
of a projective variety. Alternatively, Deligne-Mumford [20;62] constructed $\overline{\mathcal{M}}_{g}$ algebraically and showed that it is a coarse moduli space for stable genus $g$ curves, and Knudsen-Mumford [41] showed that $\overline{\mathcal{M}}_{g}$ is a projective variety.

Jacobians in genus two By associating a Riemann surface to its Jacobian, there is a natural morphism Jac: $\mathcal{M}_{g} \rightarrow \mathcal{A}_{g}$.

Theorem 5.3 [66] The morphism Jac extends to a morphism

$$
\overline{\text { Jac }:} \overline{\mathcal{M}}_{g} \rightarrow \widehat{\mathcal{A}}_{g},
$$

which sends a Riemann surface $X$ to the Jacobian of the normalization of $X$.

Determining the image of Jac map is in general very difficult; however, this is simple in genus two. Let $\widetilde{\mathcal{M}}_{2}$ be the subvariety of $\overline{\mathcal{M}}_{2}$ consisting of nonsingular Riemann surfaces together with pairs of elliptic curves joined at a single node. The following proposition is well known.

Proposition 5.4 The image of Jac: $\mathcal{M}_{2} \rightarrow \mathcal{A}_{2}$ is exactly those Abelian varieties which are not polarized products of elliptic curves. Furthermore, Jac extends to an isomorphism $\widetilde{\mathrm{Jac}}: \widetilde{\mathcal{M}_{2}} \rightarrow \mathcal{A}_{2}$.

Sketch of proof The image of Jac contains an open set because these varieties have the same dimension, and Jac restricted to $\mathcal{M}_{2}$ is injective by the Torelli theorem. Any map between complete irreducible varieties of the same dimension whose image contains an open set is onto, so $\overline{\mathrm{Jac}}$ is onto.

It's easy to check that the Jacobian of a genus two Riemann surface $X$ can't be the polarized product of two elliptic curves (for example this would give a degree one map of $X$ to an elliptic curve), so the image of $\mathcal{M}_{2}$ is exactly the complement of the locus of polarized products.

The map $\widetilde{\mathrm{Jac}}$ is also injective (since the Jacobian of a Riemann surface $X$ formed from two elliptic curves joined at a node is the product of those curves), so it is a bijection. It follows that $\widetilde{\mathrm{Jac}}$ is an isomorphism because it is a bijection between two normal varieties.

Abelian differentials over $\overline{\mathcal{M}}_{2}[g]$ The bundle $\Omega \mathcal{T}\left(\Sigma_{g}\right)$ extends to a trivial bundle $\Omega \overline{\mathcal{T}}\left(\Sigma_{g}\right)$ over $\overline{\mathcal{T}}\left(\Sigma_{g}\right)$. The quotient,

$$
\Omega \overline{\mathcal{M}}_{g}=\Omega \overline{\mathcal{T}}\left(\Sigma_{g}\right) / \operatorname{Mod}\left(\Sigma_{g}\right),
$$


is the moduli space of stable Abelian differentials of genus $g$. It is an orbifold vector bundle over $\overline{\mathcal{M}}_{g}$ whose fiber over $X$ is $(\Omega(X) \backslash\{0\}) / \operatorname{Aut}(X)$.

Similarly, define the bundle $\Omega \mathcal{D}\left(\Sigma_{g}, S\right)$ to be the restriction of

$$
\Omega \overline{\mathcal{T}}\left(\Sigma_{g}, S\right) / \operatorname{Tw}(S)
$$

to $\mathcal{D}\left(\Sigma_{g}, S\right)$. This is a trivial bundle whose fiber over a marked, stable Abelian differential $(f, X)$ is $\Omega(X)$. To see that it is trivial, choose a Lagrangian subspace $\Lambda \subset H_{1}\left(\Sigma_{g} ; \mathbb{Z}\right)$ which is orthogonal to the homology class of each of the curves in $S$. Then $\Lambda$ is fixed pointwise by the action of $\operatorname{Tw}(S)$, and

$$
\Omega \mathcal{D}\left(\Sigma_{g}, S\right) \cong \mathcal{D}\left(\Sigma_{g}, S\right) \times \operatorname{Hom}(\Lambda, \mathbb{C}) .
$$

\subsection{Degenerating Abelian differentials}

We now study in more detail the geometry of Abelian differentials as they approach the boundary of moduli space. For example, we will see that Abelian differentials close to a stable Abelian differential $(X, \omega)$ with a polar node develop very long cylinders which are pinched off to form a node in the limit. We will then use our understanding of this geometry to study the behavior of Jacobians of Riemann surfaces near the boundary of moduli space. Finally, we will give two applications concerning real multiplication which will be used in Section 7.

Long cylinders The conformal topology on $\overline{\mathcal{T}}\left(\Sigma_{g}\right)$ defined in Section 5.2 is compatible with the topology on the trivial bundle $\Omega \overline{\mathcal{T}}\left(\Sigma_{g}\right)$ in the following sense. Consider $\left[f: \Sigma_{g} \rightarrow X\right] \in \partial \mathcal{T}\left(\Sigma_{g}\right)$ with $\omega \in \Omega(X)$ nonzero. Let $U \subset X$ be a neighborhood of the nodes of $X$, and let $K>1$. Let $V_{U, K}$ be the set of all $\left(\left[g: \Sigma_{g} \rightarrow Y\right], \eta\right) \in \Omega \overline{\mathcal{T}}\left(\Sigma_{g}\right)$ for which $f$ and $g$ can be adjusted by homotopies so that $\left.g \circ f^{-1}\right|_{X \backslash \bar{U}}$ is conformal, and

$$
K^{-1}<\left|\frac{\left(g \circ f^{-1}\right)^{*} \eta}{\omega}\right|<K
$$

on $X \backslash \bar{U}$. It is an unpublished result that the $V_{U, K}$ are a neighborhood basis of $\left(\left[f: \Sigma_{g} \rightarrow X\right], \omega\right)$ in $\Omega \overline{\mathcal{T}}\left(\Sigma_{g}\right)$.

We can use this description of the topology on $\Omega \overline{\mathcal{T}}\left(\Sigma_{g}\right)$ to prove the following theorem which gives a more precise description of the shape of an Abelian differential close to a stable Abelian differential $(X, \omega)$ with $X \in \partial \mathcal{M}_{2}$.

Theorem 5.5 Let $(X, \omega) \in \Omega \overline{\mathcal{T}}\left(\Sigma_{g}\right)$ with $X \in \mathcal{T}\left(\Sigma_{g}, S\right)$, and let $C \subset X$ be a compact subset disjoint from the nodes. For any $\epsilon, h>0$ and $K>1$, there is a neighborhood $U$ of $(X, \omega)$ in $\Omega \overline{\mathcal{T}}\left(\Sigma_{g}\right)$ such that each $(Y, \eta) \in U$ has the following properties: 
- The collapse $Y \rightarrow X$ induced by the markings is homotopic to a collapse $f: Y \rightarrow X$ which is conformal on $C$ and satisfies

$$
K^{-1}<\left|\frac{f^{*} \omega}{\eta}\right|<K
$$

on $C$.

- Each curve in $S$ which represents a polar node of $(X, \omega)$ is homotopic to a cylinder on $Y$ of height at least $h$.

- Suppose that $\gamma$ is a curve in $S$ which represents a node $n$ of $X$ which is nonseparating and such that $\omega$ does not vanish at either of the points identified to form $n$. If

$$
\int_{\gamma} \eta=0
$$

then there are saddle connections $I_{1}$ and $I_{2}$ on $(Y, \eta)$ joining two distinct zeros of $\eta$ of length less than $\epsilon$ such that $\gamma$ is homotopic to $I_{1} \cup I_{2}$ and $(Y, \eta)$ can be split along $I_{1} \cup I_{2}$.

Proof The first statement follows directly from the definition of the conformal topology on $\Omega \overline{\mathcal{T}}\left(\Sigma_{g}\right)$ above.

Now let $p$ be a polar node of $(X, \omega)$. By possibly enlarging the compact set $C$, we can suppose that $C$ contains a cylinder $D$ of height $2 h$ which is homotopic to a curve going around $p$. If $f: Y \rightarrow X$ is a collapse satisfying (5-2) with $K<2$, then the two boundary components of $f^{-1}(D)$ are at least distance $h$ apart. Furthermore, if $K$ is sufficiently small, then the boundary components of $f^{-1}(D)$ both have winding number zero, so $f^{-1}(D)$ contains no zeros. Therefore $f^{-1}(D)$ contains a cylinder of height at least $h$, which proves the second statement.

Now suppose that $p$ is a holomorphic node of $(X, \omega)$ and suppose that (5-3) holds. If $\epsilon$ is sufficiently small, then there is a neighborhood $U$ of $p$ isomorphic to two disks of radius $\epsilon / \pi$ joined at a point. By possibly enlarging the compact subset $C \subset X$, we have $\partial U \subset C$. If $\left(\left[f: \Sigma_{2} \rightarrow Y\right], \eta\right) \in V_{X \backslash C, K}$ with $K<2$, then $f^{-1}(U)$ is bounded by two curves $\beta_{i}$ of circumference less than $2 \epsilon$. Furthermore, if $K$ is sufficiently small, then the derivatives of $f$ will be close to the identity, and we can assume that the curves $\beta_{i}$ have winding number one and positive curvature. Since the $\beta_{i}$ have winding number one, it follows from the Gauss-Bonnet Theorem that $\eta$ has two zeros in $f^{-1}(U)$, counting multiplicity. Since the $\beta_{i}$ have positive curvature, there is a shortest geodesic $\delta \in f^{-1}(U)$ generating $\pi_{1}\left(f^{-1}(U)\right)$. By (5-3), we have

$$
\int_{\delta} \eta=0
$$


which implies that $\delta$ is not a straight geodesic, so it must be a union of saddle connections joining distinct zeros. As there are at most two zeros in $f^{-1}(U)$, the curve $\delta$ must be a union of two saddle connections $I_{1}$ and $I_{2}$ going from a zero $q$ to a zero $r$ of $\eta$. By (5-4), we have

$$
\int_{I_{1}} \eta=\int_{I_{2}} \eta
$$

This means that we can split along $I_{1} \cup I_{2}$ by the discussion of splitting along a union of saddle connections in Section 4.1. Finally, since $\delta$ is the shortest curve in its homotopy class in $f^{-1}(U)$ and the length of $\beta_{i}$ is less than $2 \epsilon$, the length of $I_{i}$ must be less than $\epsilon$ as claimed.

Semi-Abelian varieties We now introduce a topology on the space of semi-Abelian varieties and show that the map which associates to a marked stable Riemann surface its Jacobian is continuous.

A semi complex torus is a quotient $A=V / \Lambda$, where $V$ is a complex vector space containing a discrete subgroup $\Lambda$, such that there is an exact sequence,

$$
0 \rightarrow\left(\mathbb{C}^{*}\right)^{n} \rightarrow A \stackrel{\phi}{\rightarrow} B \rightarrow 0,
$$

where $B=V^{\prime} / \Lambda^{\prime}$ is a compact complex torus. A principal polarization $h^{\prime}$ on $B$ induces a degenerate Hermitian form $h$ on $V$ by pulling back $h^{\prime}$ by the lift $\tilde{\phi}: V \rightarrow V^{\prime}$. Such a Hermitian form on $V$ is a principal polarization on $A$. S semi-Abelian variety is a semi complex torus together with a principal polarization.

Equip $\mathbb{Z}^{2 g}$ with the usual symplectic form defined by (2-1). A marked semi-Abelian variety is a triple $(A, \Gamma, \phi)$, where:

- $A$ is a semi-Abelian variety.

- $\Gamma \subset \mathbb{Z}^{2 g}$ is a subgroup of $\mathbb{Z}^{2 g}$ on which the symplectic form vanishes and which is saturated in the sense that if $x \in \mathbb{Z}^{2 g}$ and $m x \in \Gamma$ for some $m \in \mathbb{Z}$, then $x \in \Gamma$.

- $\phi$ is a symplectic isomorphism $\delta: \Gamma^{\perp} \rightarrow H_{1}(A ; \mathbb{Z})$.

Let $\mathcal{H}_{g}^{\dagger}$ be the space of all marked semi-Abelian varieties. The subspace of $\mathcal{H}_{g}^{\dagger}$ consisting of marked Abelian varieties is naturally isomorphic to the Siegel upper half plane $\mathcal{H}_{g}$ by the discussion is Section 2.1.

We give $\mathcal{H}_{g}^{\dagger}$ a topology as follows. If

$$
\left(A_{n}=V_{n} / \Lambda_{n}, \Gamma_{n}, \phi_{n}\right)
$$


is a sequence in $\mathcal{H}_{g}^{\dagger}$, then we say that this sequence converges to $(A=V / \Lambda, \Gamma, \phi)$ if eventually $\Gamma_{n} \subset \Gamma$ and there are linear isomorphisms $\psi_{n}: V_{n} \rightarrow V$ with the following properties:

- For all $\alpha \in \Gamma^{\perp}$,

$$
\lim _{n \rightarrow \infty} \psi_{n} \circ \phi_{n}(\alpha)=\phi(\alpha) .
$$

- For all $\alpha \in \mathbb{Z}^{2 g} \backslash \Gamma^{\perp}$, the sequence $\psi_{n} \circ \phi_{n}(\alpha)$ eventually leaves every compact subset of $V$.

- If $h_{n}$ and $h$ are the polarizations on $A_{n}$ and $A$, then

$$
\lim _{n \rightarrow \infty}\left(\phi_{n}\right)_{*}\left(h_{n}\right)=h
$$

as Hermitian forms on $V$.

This defines a Hausdorff topology on $\mathcal{H}_{g}^{\dagger}$.

Over $\mathcal{H}_{g}^{\dagger}$ is the trivial rank-two bundle $\Omega \mathcal{H}_{g}^{\dagger}$ consisting of pairs $(A, \omega)$, where $A \in \mathcal{H}_{g}^{\dagger}$ and $\omega \in \Omega(A)$, the space of holomorphic one-forms on $A$.

A choice of a symplectic isomorphism $H_{1}\left(\Sigma_{2} ; \mathbb{Z}\right) \rightarrow \mathbb{Z}^{4}$ defines natural maps

$$
\text { Jac: } \overline{\mathcal{T}}\left(\Sigma_{2}\right) \rightarrow \mathcal{H}_{2}^{\dagger} \quad \text { and } \quad \Omega \text { Jac: } \Omega \overline{\mathcal{T}}\left(\Sigma_{2}\right) \rightarrow \Omega \mathcal{H}_{2}^{\dagger} \text {, }
$$

sending a marked stable Riemann surface to its Jacobian with the induced marking.

Theorem 5.6 The maps Jac and $\Omega$ Jac defined above are continuous.

We will sketch the proof of this theorem below.

Given any $X \in \mathcal{T}\left(\Sigma_{2}, S\right)$, we can define a norm $\|\cdot\|_{X}$ on $H_{1}\left(\Sigma_{2} ; \mathbb{R}\right)$, the Hodge norm as follows. Let $V_{S} \subset H_{1}\left(\Sigma_{2} ; \mathbb{R}\right)$ be the subspace generated by the curves in $S$. If $\gamma \notin V_{S}^{\perp} \subset H_{1}\left(\Sigma_{2} ; \mathbb{R}\right)$, set $\|\gamma\|_{X}=\infty$. On $V_{S}^{\perp}$, let $\|\cdot\|_{X}$ be the norm induced by the Hermitian metric on $\Omega(X)^{*}$ coming from the polarization via the embedding $V_{S}^{\perp} \rightarrow \Omega(X)^{*}$. Alternatively, for $\gamma \in V_{S}^{\perp}$,

$$
\begin{gathered}
\|\gamma\|_{X}=\sup _{\substack{\omega \in \Omega(X) \\
\|\omega\|=1}}|\omega(\gamma)|, \\
\|\omega\|=\left(\int_{X}|\omega|^{2}\right)^{1 / 2} .
\end{gathered}
$$

where

Theorem 5.7 Let $V_{S, \mathbb{Z}} \subset H_{1}\left(\Sigma_{2} ; \mathbb{Z}\right)$ be the subgroup generated by the curves in $S$. If $\left\{X_{n}\right\}$ is a sequence in $\overline{\mathcal{T}}\left(\Sigma_{2}\right)$ converging to $X$ in $\mathcal{T}\left(\Sigma_{2}, S\right)$, then: 
- There exists a $C>0$ such that $\|\gamma\|_{X_{n}}>C$ for all $\gamma \in V_{S, \mathbb{Z}}^{\perp} \backslash V_{S ; \mathbb{Z}}$.

- For all $D>0$, there exists $N>0$ such that $\|\gamma\|_{X_{n}}>D$ for all $\gamma \in H_{1}\left(\Sigma_{2} ; \mathbb{Z}\right) \backslash$ $V_{S, \mathbb{Z}}^{\perp}$ if $n>N$.

The proof of these theorems will rely on the following lemma.

Lemma 5.8 Given any linear map $R: V_{S} \rightarrow \mathbb{R}$, there is a unique section $X \mapsto \omega_{X}$ of $\Omega \overline{\mathcal{T}}\left(\Sigma_{2}, S\right)$ over $\overline{\mathcal{T}}\left(\Sigma_{2}, S\right)$ (defined in $\left.(5-1)\right)$ such that:

- $\omega_{X}(\gamma)=R(\gamma)$ for all $\gamma \in V_{S}$.

- $\operatorname{Im} \omega_{X}(\gamma)=0$ for all $\gamma \in V_{S}^{\perp}$.

Proof Let $X \in \overline{\mathcal{T}}\left(\Sigma_{2}, S\right)$. We have an exact sequence,

$$
0 \rightarrow \Omega(\tilde{X}) \rightarrow \Omega(X) \rightarrow \operatorname{Hom}\left(V_{S}, \mathbb{C}\right) \rightarrow 0,
$$

where $\tilde{X}$ is the normalization of $X$. Choose some $\eta \in \Omega(X)$ such that $\omega(\gamma)=R(\gamma)$ for each $\gamma \in V_{S}$. The form $\eta$ defines a map,

$$
S: V_{S}^{\perp} / V_{S} \rightarrow \mathbb{R}
$$

by $S(\gamma)=\operatorname{Im} \eta(\gamma)$. We have isomorphisms of real vector spaces,

$$
H_{1}(\tilde{X} ; \mathbb{R}) \rightarrow V_{S}^{\perp} / V_{S},
$$

and

$$
\Omega(\tilde{X}) \rightarrow \operatorname{Hom}\left(H_{1}(\tilde{X} ; \mathbb{R}), \mathbb{R}\right),
$$

defined by $\omega \mapsto(\gamma \mapsto \operatorname{Im} \omega(\gamma))$. Let $v \in \Omega(\tilde{X})$ induce the map $S$ above. Then $\omega_{X}=\eta-v$ is the desired form, giving the desired section over $\overline{\mathcal{T}}\left(\Sigma_{2}, S\right)$.

Sketch of Proof of Theorem 5.6 Suppose we have a sequence $\left\{X_{n}\right\}$ in $\overline{\mathcal{T}}\left(\Sigma_{2}\right)$ converging to some $X \in \mathcal{T}\left(\Sigma_{2}, S\right)$. To prove that Jac is continuous, we need to show:

- For every section $Y \mapsto \omega_{Y}$ of $\Omega \overline{\mathcal{T}}\left(\Sigma_{2}, S\right)$ and $\gamma \in V_{S}^{\perp}$, we have

$$
\omega_{X_{n}}(\gamma) \rightarrow \omega_{X}(\gamma)
$$

- For every $\gamma \in H_{1}\left(\Sigma_{2} ; \mathbb{Z}\right) \backslash V_{S}^{\perp}$, there is a section $Y \mapsto \eta_{Y}$ of $\Omega \overline{\mathcal{T}}\left(\Sigma_{2}, S\right)$ such that $\left|\eta_{X_{n}}(\gamma)\right| \rightarrow \infty$.

We leave the proof that the polarizations converge to the reader.

If $X \mapsto \omega_{X}$ is a section of $\Omega \overline{\mathcal{T}}\left(\Sigma_{2}, S\right)$, then $\omega_{X_{n}}$ converges uniformly to $\omega_{X}$ away from the nodes of $X$ as in Theorem 5.5. If $\gamma \in V_{S}^{\perp}$, then $\gamma$ is represented by a curve on $X$ which is disjoint from the nodes, so $\omega_{X_{n}}(\gamma) \rightarrow \omega(\gamma)$ by uniform convergence. 
Suppose $\gamma \in H_{1}\left(\Sigma_{2} ; \mathbb{Z}\right) \backslash V_{S}^{\perp}$. By Lemma 5.8, there is a section $X \mapsto \eta_{X}$ of $\Omega \overline{\mathcal{T}}\left(\Sigma_{2}, S\right)$ such that

$$
\operatorname{sign} \eta(\alpha)=\operatorname{sign}(\alpha \cdot \gamma)
$$

for every $\alpha \in H_{1}\left(\Sigma_{2} ; \mathbb{Z}\right)$ representing a curve of $S$. If $n$ is large, then each $\alpha \in$ $H_{1}\left(\Sigma_{2} ; \mathbb{Z}\right)$ representing a curve of $S$ is homologous to a very tall cylinder $C_{\alpha}$ on $\left(X_{n}, \eta_{X_{n}}\right)$, and this cylinder contributes positively to $\operatorname{Im} \eta_{X_{n}}(\gamma)$. If $n$ is sufficiently large, then the contributions from these cylinders will be much greater then that from the rest of $\left(X_{n}, \eta_{X_{n}}\right)$, and we will have $\operatorname{Im} \eta_{X_{n}}(\gamma) \rightarrow \infty$.

Proof of Theorem 5.7 Let $\left\{\eta^{i}\right\}$ be a basis of $\Omega(\tilde{X})$ such that $\left\|\eta^{i}\right\|=1$. There is some $C^{\prime}>0$ such that

$$
\sup _{i}\left|\eta^{i}(\gamma)\right|>C^{\prime}
$$

for all $\gamma \in V_{S, \mathbb{Z}}^{\perp} / V_{S, \mathbb{Z}}$. Let

$$
C=\frac{1}{2} \sup _{i} C_{i}
$$

For each $i$, there is a sequence $\eta_{n}^{i} \in \Omega\left(X_{n}\right)$ such that

$$
\eta_{n}^{i} \rightarrow \eta^{i}
$$

By Theorem 5.5, we have

$$
\left\|\eta_{n}^{i}\right\| \rightarrow\left\|\eta^{i}\right\|
$$

so we can normalize $\eta_{n}^{i}$ so that $\left\|\eta_{n}^{i}\right\|=1$ for all $n$, and (5-6) still holds. By the uniform convergence statement of Theorem 5.5, we have for each $i$ and $\gamma \in V_{S, \mathbb{Z}}^{\perp}$,

$$
\left|\eta_{n}^{i}(\gamma)\right|>\frac{1}{2}\left|\eta^{i}(\gamma)\right|
$$

for $n$ sufficiently large. The first claim of the Theorem follows.

Let $\alpha_{i} \in H_{1}\left(\Sigma_{2} ; \mathbb{Z}\right)$ be homology classes representing each nonseparating curve $S_{i}$ of $S$. Choose a section $Y \mapsto \omega_{Y}$ of $\Omega \overline{\mathcal{T}}\left(\Sigma_{2} ; S\right)$ as in Lemma 5.8 such that

$$
\omega_{Y}\left(\alpha_{i}\right) \in \mathbb{R} \backslash\{0\}
$$

for each $i$. We claim that for every $C>0$ and $\gamma \in H_{1}\left(\Sigma_{2} ; \mathbb{Z}\right) \backslash V_{S, \mathbb{Z}}^{\perp}$ such that

$$
\operatorname{sign} \alpha \cdot \gamma=\operatorname{sign} \omega_{X}(\alpha),
$$

there is some $N$ for which we have

$$
\frac{\left|\omega_{X_{n}}(\gamma)\right|}{\left\|\omega_{X_{n}}\right\|}>C
$$


for all $n>N$. The second claim of the Theorem follows because we can choose finitely many such sections so that for all $\gamma \in H_{1}\left(\Sigma_{2} ; \mathbb{Z}\right) \backslash V_{S, \mathbb{Z}}^{\perp},(5-7)$ holds for some section.

Let $M \subset X$ be a compact subset which is a deformation retract of the complement of the nodes. By Theorem 5.5, there are collapses $g_{n}: X_{n} \rightarrow X$ such that

$$
K_{n}^{-1}<\left|\frac{\left(g_{n}\right)^{*} \omega_{X}}{\omega_{X_{n}}}\right|<K_{n}
$$

on $M_{n}=g_{n}^{-1}(M)$ with $K_{n} \geq 1$ and $K_{n} \rightarrow 1$. There are also cylinders $C_{i, n}$ in $X_{n} \backslash M_{n}$ which represent the homology classes $\alpha_{i}$ such that if $H_{i, n}=$ height $C_{i, n}$, then $\lim _{n \rightarrow \infty} H_{i, n}=\infty$.

The class $\gamma$ is represented on each $X_{n}$ by a closed curve $\gamma_{n}$. On $X_{n}$, each cylinder $C_{i, n}$ contributes

$$
\left|\alpha_{i} \cdot \gamma\right| H_{i, n}
$$

to $\operatorname{Im} \int_{\gamma} \omega_{X_{n}}$. There are also segments of $\gamma_{n}$ which pass through $M_{n}$ to get from one cylinder to another. Let $\beta$ be such a segment. Since we chose $\omega_{X_{n}}$ so that its periods over $V_{S, \mathbb{Z}}^{\perp}$ are all real, $\operatorname{Im} \int_{\beta} \omega_{X_{n}}$ doesn't depend on the path $\beta$ takes. If $n$ is large, then this integral will be uniformly bounded over each such path $\beta$, say by

$$
\left|\operatorname{Im} \int_{\beta} \omega_{X_{n}}\right|<c=\operatorname{diameter}(M)+1 .
$$

We have $\left\|\omega_{X_{n}}\right\|=\left(\sum_{i} H_{i, n}+\operatorname{Area}(M)\right)^{1 / 2} \leq\left(r \max _{i} H_{i, n}+\operatorname{Area}(M)\right)^{1 / 2}$,

where $r$ is the number of curves in $S$. Also, we have

$$
\operatorname{Im} \int_{\gamma_{n}} \omega \geq \max _{i} H_{i, n}-c .
$$

Thus $\quad\|\gamma\|_{X_{n}} \geq \frac{\left|\int_{\gamma_{n}} \omega_{X_{n}}\right|}{\left\|\omega_{X_{n}}\right\|} \geq \frac{\operatorname{Im} \int_{\gamma_{n}} \omega_{X_{n}}}{\left\|\omega_{X_{n}}\right\|} \geq \frac{\max _{i} H_{i, n}-c}{\sqrt{r \max _{i} H_{i, n}+\operatorname{Area}(M)}} \rightarrow \infty$ as $n \rightarrow \infty$.

Real multiplication near $\mathbf{\partial} \mathcal{M}_{2}$ We now use the two previous theorems to study eigenforms for real multiplication near $\partial \mathcal{M}_{2}$. As in the previous paragraph, we will write $V_{S, \mathbb{Z}}$ for the subgroup of $H_{1}\left(\Sigma_{2} ; \mathbb{Z}\right)$ generated by the curves in a curve system $S$ on $\Sigma_{2}$. 
We can define real multiplication on semi-Abelian varieties just as we did for Abelian varieties in Section 2.2; however, we will not require real multiplication on a semiAbelian variety to be proper.

Theorem 5.9 Let $X \in \mathcal{T}\left(\Sigma_{2}, S\right)$ be a stable Riemann surface whose Jacobian has real multiplication by $\mathcal{O}_{D}$. There is a neighborhood $U$ of $X$ in $\overline{\mathcal{T}}\left(\Sigma_{2}\right)$ such that for each stable Riemann surface $Y \in U$ which has real multiplication by $\mathcal{O}_{D}$, the real multiplication preserves the two subspaces $V_{S, \mathbb{Z}}$ and $V_{S, \mathbb{Z}}^{\perp}$ of $H_{1}\left(\Sigma_{2} ; \mathbb{Z}\right)$.

Proof Suppose $\left\{X_{n}\right\} \subset \overline{\mathcal{T}}\left(\Sigma_{2}\right)$ is a sequence converging to $X$. If $\gamma \in V_{S, \mathbb{Z}}$, then we have $\|\gamma\|_{X}=0$, so $\|\gamma\|_{X_{n}} \rightarrow 0$ as $n \rightarrow \infty$. Let $\rho_{n}: \mathcal{O}_{D} \rightarrow \operatorname{End} \operatorname{Jac}\left(X_{n}\right)$ be a choice of real multiplication, and let $\lambda \in \mathcal{O}_{D}$ be a generator of $\mathcal{O}_{D}$ over $\mathbb{Z}$. If the claim of the Theorem is false, then taking a subsequence, we can assume that $\rho_{n}(\lambda) \cdot \gamma \notin V_{S, \mathbb{Z}}$ for all $n$. We have

$$
\left\|\rho_{n}(\lambda) \cdot \gamma\right\|_{X_{n}} \leq \sup _{i}\left|\lambda^{(i)}\right| \cdot\|\gamma\|_{X_{n}} .
$$

This is a contradiction because the right hand side of this equation goes to zero while the left hand side is bounded below by Theorem 5.7.

The assertion that $V_{S, \mathbb{Z}}^{\perp}$ is preserved is proved in the same way.

Recall that we have the subspace $\Omega E_{D} \subset \Omega \mathcal{M}_{2}$ of eigenforms for real multiplication by $\mathcal{O}_{D}$. Let $\overline{\Omega E}_{D} \subset \Omega \overline{\mathcal{M}}_{2}$ be the closure of $\Omega E_{D}$.

Theorem 5.10 Every stable Abelian differential $(X, \omega) \in \overline{\Omega E}_{D}$ is a (not necessarily proper) eigenform for real multiplication by $\mathcal{O}_{D}$.

Proof Suppose $\left(X_{n}, \omega_{n}^{1}\right)$ is a sequence in $\Omega \mathcal{T}\left(\Sigma_{2}\right)$ is a sequence of 1-eigenforms for real multiplication by $\mathcal{O}_{D}$ converging to some $\left(X, \omega^{1}\right)$ with $X \in \mathcal{T}\left(\Sigma_{2}, S\right)$. Let $\omega_{n}^{2} \in \Omega\left(X_{n}\right)$ be a sequence of 2-eigenforms. Taking a subsequence and suitably normalizing the $\omega_{n}^{2}$, we can assume that $\omega_{n}^{2} \rightarrow \omega^{2}$ for some nonzero $\omega^{2} \in \Omega(X)$.

We claim that the $\omega^{i}$ span $\Omega(X)$. Suppose not. Then $\omega^{1}=c \omega^{2}$ for some $c \in \mathbb{C}$. Choose some $\alpha \in V_{S, \mathbb{Z}}^{\perp}$ such that $\omega^{1}(\alpha) \neq 0$, and let $\lambda \in \mathcal{O}_{D} \backslash \mathbb{Z}$ be a generator of $\mathcal{O}_{D}$ over $\mathbb{Z}$. Then for $n$ large,

$$
\lambda^{(1)} \omega^{1}(\alpha) \sim \omega_{n}^{1}\left(\rho_{n}(\lambda) \cdot \alpha\right) \sim c \omega_{n}^{2}\left(\rho_{n}(\lambda) \cdot \alpha\right)=c \lambda^{(2)} \omega_{n}^{2}(\alpha) \sim \lambda^{(2)} \omega^{1}(\alpha),
$$

a contradiction because $\lambda^{(1)} \neq \lambda^{(2)}$. 
Now via the dual bases to $\left\{\omega_{n}^{i}\right\}$ and $\left\{\omega^{i}\right\}$, we can identify $\Omega\left(X_{n}\right)^{*}$ and $\Omega(X)^{*}$ with $\mathbb{C}^{2}$. We then have natural embeddings

and

$$
\phi_{n}: H_{1}\left(\Sigma_{2} ; \mathbb{Z}\right) \rightarrow \mathbb{C}^{2},
$$

$$
\phi: V_{S, \mathbb{Z}}^{\perp} \rightarrow \mathbb{C}^{2}
$$

such that

$$
\begin{aligned}
\operatorname{Jac}\left(X_{n}\right) & \cong \mathbb{C}^{2} / \operatorname{Im} \phi_{n}, \\
\operatorname{Jac}(X) & \cong \mathbb{C}^{2} / \operatorname{Im} \phi,
\end{aligned}
$$

and

and $\phi_{n} \rightarrow \phi$ as $n \rightarrow \infty$. The real multiplication on $\operatorname{Jac}\left(X_{n}\right)$ lifts to

$$
\tilde{\rho}\left(z_{1}, z_{2}\right)=\left(\lambda^{(1)} z_{1}, \lambda^{(2)} z_{2}\right)
$$

on $\mathbb{C}^{2}$. Since $\phi_{n} \rightarrow \phi$, the map $\tilde{\rho}$ preserves $\operatorname{Im} \phi$, so defines real multiplication on $\operatorname{Jac}(X)$ with $\omega^{1}$ an eigenform as desired.

\section{Local coordinates for strata in $\Omega \overline{\mathcal{M}}_{2}$}

While the stratification of $\Omega \mathcal{M}_{2}$ is very simple, consisting of only two strata, the natural stratification of $\Omega \overline{\mathcal{M}}_{2}$ is much more complicated. With the appropriate definition of a stratum, there are seventeen different strata in $\Omega \overline{\mathcal{M}}_{2}$. It will be necessary for our study of the compactification of $X_{D}$ to understand them. In particular, we will need to give local coordinates on $\Omega \overline{\mathcal{M}}_{2}$ around most of these strata. In Section 6.1, we will define the stratification of $\Omega \overline{\mathcal{M}}_{2}$, give notation for the various strata, and list the seventeen strata in $\Omega \overline{\mathcal{M}}_{2}$. In the rest of this section, we will then discuss each stratum in turn.

\subsection{Strata in $\Omega \overline{\mathcal{M}}_{2}$}

Given two stable Abelian differentials $\left(X_{1}, \omega_{1}\right)$ and $\left(X_{2}, \omega_{2}\right) \in \Omega \overline{\mathcal{M}}_{2}$, say that they are in the same stratum of $\Omega \overline{\mathcal{M}}_{2}$ if there is a homeomorphism $f: X_{1} \rightarrow X_{2}$ with the following properties:

- $f$ takes zeros of $\omega_{1}$ to zeros of $\omega_{2}$ of the same order.

- $f$ takes polar nodes of $\left(X_{1}, \omega_{1}\right)$ to polar nodes of $\left(X_{2}, \omega_{2}\right)$.

- $f$ takes irreducible components of $X_{1}$ on which $\omega_{1}$ vanishes to irreducible components of $X_{2}$ on which $\omega_{2}$ vanishes. 
Notation for strata We now introduce notation for the various strata in $\Omega \overline{\mathcal{M}}_{2}$. Let $S \subset \Sigma_{2}$ be a curve system, and define $\Omega \mathcal{T}_{2}^{0}(S)$ to be the locus of all $(X, \omega) \in \Omega \overline{\mathcal{T}}_{2}$ such that:

- $X \in \mathcal{T}_{2}(S)$.

- The form $\omega$ has poles at the nodes corresponding to nonseparating curves in $S$.

If $T \in S$ is a nonseparating curve, then define $\Omega \mathcal{T}_{2}^{0}(S, T)$ to be the locus of all $(X, \omega) \in \Omega \overline{\mathcal{T}}_{2}$ such that:

- $X \in \mathcal{T}_{2}(S)$.

- The form $\omega$ has poles at the nodes corresponding to nonseparating curves in $S \backslash T$.

- The form $\omega$ is holomorphic at the node corresponding to the curve $T$.

If $\mathbf{n}$ is either (2) or $(1,1)$, then let

$$
\Omega \mathcal{T}_{2}^{0}(S ; \mathbf{n}) \subset \Omega \mathcal{T}_{2}^{0}(S)
$$

be the locus of $(X, \omega) \in \Omega \mathcal{T}_{2}^{0}(S)$ where in addition, $\omega$ has zeros whose orders are given by $\mathbf{n}$.

Let $\Omega \mathcal{M}_{2}^{0}(S, T)$ and $\Omega \mathcal{M}_{2}^{0}(S)$ and $\Omega \mathcal{M}_{2}^{0}(S ; \mathbf{n})$ be the images in $\Omega \mathcal{M}_{2}(S)$ of the corresponding spaces defined above.

Let $\Omega \mathcal{D}_{2}^{\prime}(S, T)$ be the locus of Abelian differentials $(X, \omega) \in \Omega \mathcal{D}_{2}(S)$ such that either $\int_{T} \omega=0$ or $T$ represents a holomorphic node on $(X, \omega)$. This is a nonsingular hypersurface in $\Omega \mathcal{D}_{2}(S)$.

Let $\Omega \mathcal{D}_{2}^{0}(S)$ be the locus of Abelian differentials $(X, \omega) \in \Omega \mathcal{D}_{2}(S)$ such that each nonseparating curve of $S \backslash T$ is represented by either a polar node of $(X, \omega)$ or a cylinder on $(X, \omega)$. Given a curve $T \in S$, let

$$
\Omega \mathcal{D}_{2}^{0}(S, T) \subset \Omega \mathcal{D}_{2}^{\prime}(S, T)
$$

be the locus of $(X, \omega)$ with the following properties:

- Each nonseparating curve of $S \backslash T$ is represented by either a polar node of $(X, \omega)$ or a cylinder on $(X, \omega)$.

- A curve $\gamma$ which is either $T$ or a separating curve in $S$ is either represented by a holomorphic node of $(X, \omega)$ or is homotopic to a union $I \cup J(I)$ of a saddle connection $I$ with its image under the hyperelliptic involution $J$. 
By Theorem 5.5, $\Omega \mathcal{D}_{2}^{0}(S, T)$ is a neighborhood of $\Omega \mathcal{T}_{2}^{0}(S, T)$ in $\Omega \mathcal{D}_{0}^{\prime}(S, T)$.

Also, let

$$
\Omega \mathcal{D}_{2}^{0}(S, \mathbf{n}) \subset \Omega \mathcal{D}_{2}^{0}(S)
$$

be those $(X, \omega) \in \Omega \mathcal{D}_{2}^{0}(S)$ such that $\omega$ has zeros whose orders are given by $\mathbf{n}$.

Up to the action of the mapping class group, there are six curve systems in $\Sigma_{2}$, shown in Figure 7. We will denote by $T_{i, j}$ a curve system with $i$ separating curves and $j$ nonseparating curves. Such a curve system is unique up to the action of the mapping class group. We will write $T_{i, j}^{k}$ with $k=1, \ldots n$ for the $n$ individual curves of $T_{i, j}$, and we will always order the curves so that if $T_{i, j}$ contains a separating curve, then it is the last curve $T_{i, j}^{n}$.
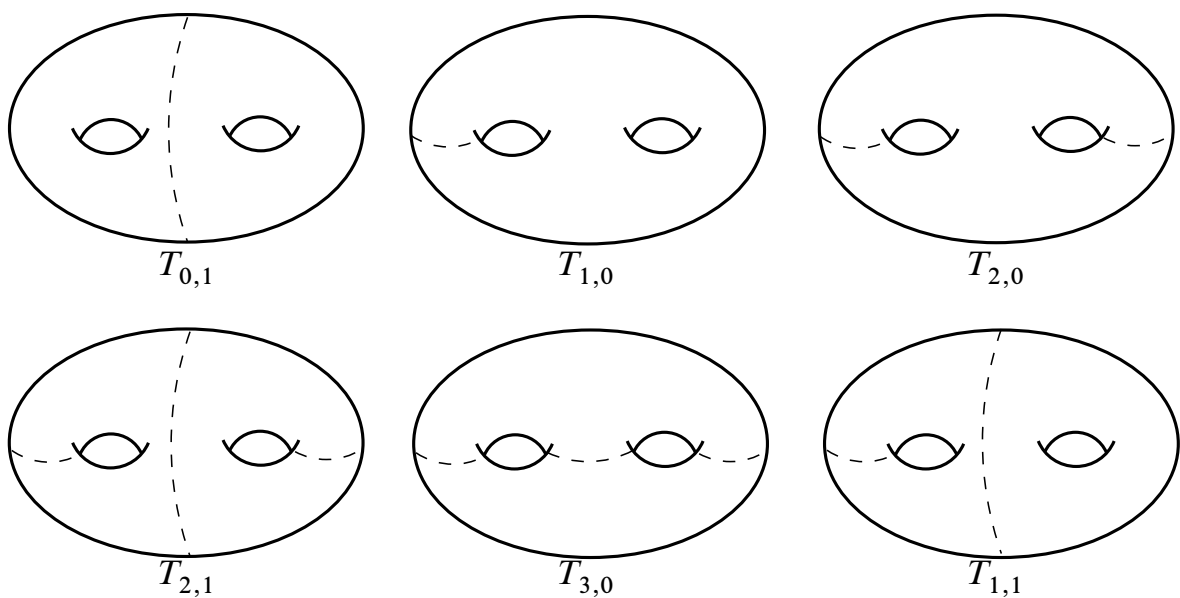

Figure 7: Six curve systems on $\Sigma_{2}$

The seventeen strata Here is a list of the seventeen strata in $\Omega \overline{\mathcal{M}}_{2}$.

(1) $\Omega \mathcal{M}_{2}(1,1)$ is the stratum of nonsingular Abelian differentials with two simple zeros.

(2) $\Omega \mathcal{M}_{2}(2)$ is the stratum of nonsingular Abelian differentials with a double zero.

(3) $\Omega \mathcal{M}_{2}^{0}\left(T_{0,1}\right)$ is the stratum of stable Abelian differentials consisting of two nonzero genus one Abelian differentials joined at a node.

(4) $\Omega \mathcal{M}_{2}^{0}\left(T_{1,0} ; 1,1\right)$ is the stratum of stable Abelian differentials with one nonseparating polar node and two simple zeros.

(5) $\Omega \mathcal{M}_{2}^{0}\left(T_{1,0} ; 2\right)$ is the stratum of stable Abelian differentials with one nonseparating polar node and one double zero. 
(6) $\Omega \mathcal{M}_{2}^{0}\left(T_{1,0}, T_{1,0}^{1}\right)$ is the stratum of stable Abelian differentials with one nonseparating holomorphic node. These can be regarded as genus one Abelian differentials with two points joined to a node, and they have no zeros.

(7) $\Omega \mathcal{M}_{2}^{0}\left(T_{2,0}, T_{2,0}^{2}\right)$ is the stratum of stable Abelian differentials with one nonseparating holomorphic node and one nonseparating polar node. These can be regarded as the differential $a d z / z$ on $\mathbb{C}^{*}$ with the points 0 and $\infty$ identified to form a polar node, and with two points in $\mathbb{C}^{*}$ identified to form a holomorphic node.

(8) $\Omega \mathcal{M}_{2}^{0}\left(T_{2,0} ; 1,1\right)$ is the stratum of stable Abelian differentials with two nonseparating polar nodes and two simple zeros.

(9) $\Omega \mathcal{M}_{2}^{0}\left(T_{2,0} ; 2\right)$ is the stratum of stable Abelian differentials with two nonseparating polar nodes and a double zero.

(10) $\Omega \mathcal{M}_{2}^{0}\left(T_{2,1}\right)$ is the stratum of stable Abelian differentials with two nonseparating polar nodes and one separating holomorphic node. These can be regarded as two infinite cylinders with the ends of the cylinders identified to form polar nodes and one point from each cylinder identified to form a holomorphic node.

(11) $\Omega \mathcal{M}_{2}^{0}\left(T_{3,0}\right)$ is the stratum of stable Abelian differentials $(X, \omega)$ with three nonseparating polar nodes. These have two simple zeros, one in each irreducible component of $X$.

(12) $\Omega \mathcal{M}_{2}^{0}\left(T_{3,0}, T_{3,0}^{3}\right)$ is the stratum of stable Abelian differentials with two nonseparating polar nodes and one nonseparating holomorphic node. These differentials have no zeros.

(13) $\Omega \mathcal{M}_{2}^{0}\left(T_{1,1}\right)$ is the stratum of stable Abelian differentials with one nonseparating polar node and one separating holomorphic node.

(14-17) There are four other strata consisting of stable Abelian differentials $(X, \omega)$ which vanish on some irreducible component of $X$. There is one such stratum corresponding to the curve system $T_{0,1}$, one corresponding to the curve system $T_{2,1}$, and two corresponding to the curve system $T_{1,1}$.

We will now go down this list and discuss each of these strata. We will not discuss the stratum $\Omega \mathcal{M}_{2}(1,1)$ because we don't need to know anything more about it, and we will not discuss the strata 13-17 on this list because they do not arise in this paper.

\subsection{The strata $\Omega \mathcal{M}_{2}(2)$ and $\Omega \mathcal{M}_{2}\left(T_{0,1}\right)$}

The strata $\Omega \mathcal{M}_{2}(2)$ and $\Omega \mathcal{M}_{2}\left(T_{0,1}\right)$ are suborbifolds of $\Omega \overline{\mathcal{M}}_{2}$. We will see this explicitly by extending the period coordinates on these strata to period coordinates on a neighborhood of these strata. 
Period coordinates for $\boldsymbol{\Omega} \mathcal{M}_{\mathbf{2}}(2)$ The map Per: $\Omega \mathcal{T}_{2} \rightarrow H^{1}\left(\Sigma_{2} ; \mathbb{C}\right)$ is a submersion by the period coordinates from Section 4.2, so the fibers of this map are the leaves of a foliation $\mathcal{A}$ of $\Omega \mathcal{T}_{2}$ by Riemann surfaces along whose leaves the absolute periods of Abelian differentials are constant. This foliation meets the stratum $\Omega \mathcal{T}_{2}(2)$ transversely because the restriction of Per to this stratum is locally biholomorphic.

We can locally parameterize the leaf $L$ of $\mathcal{A}$ through some $(X, \omega) \in \Omega \mathcal{T}_{2}(2)$ using the operation of splitting a double zero from Section 4.1 as follows. Choose $\epsilon>0$ such that every geodesic segment starting at the zero $p$ of $(X, \omega)$ of length less than $\epsilon^{3}$ is embedded in $X$. Since $p$ is a cone point of cone angle $6 \pi$, we can parameterize short geodesic segments starting at $p$ by the $\epsilon$-disk $\Delta_{\epsilon} \subset \mathbb{C}$ by associating continuously to a point $z \in \Delta_{\epsilon}$ a geodesic segment $I(z) \subset X$ such that

$$
\int_{I(z)} \omega=z^{3}
$$

The map $\phi: \Delta_{\epsilon} \rightarrow L$ defined by

$$
\phi(z)=(X, \omega) \#_{I(z)}
$$

is holomorphic and satisfies $\phi(z)=\phi(-z)$ because the segments $I(z)$ and $I(-z)$ differ by an angle of $3 \pi$, and so these segments determine the same " $X$ " in $(X, \omega)$ (see Figure 3), along which we perform surgery to form $\phi(z)$. Furthermore, $\phi(z)=\phi(w)$ if and only if $z= \pm w$, so $\phi$ lifts to a map $\tilde{\phi}$ defined by

$$
\tilde{\phi}(z)=(X, \omega) \#_{I\left(z^{1 / 2}\right)},
$$

which maps $\Delta_{\epsilon^{2}}$ conformally onto a neighborhood of $(X, \omega)$ in $L$.

Now let $U \subset \Omega \mathcal{T}_{2}(2)$ be a small neighborhood of $(X, \omega)$. We can parameterize the above construction to define continuously for each $(Y, \eta) \in U$ and $z \in \Delta_{\epsilon}$ (possibly making $\epsilon$ smaller) a geodesic segment $I_{(Y, \eta)}(z)$ on $Y$ starting at the zero of $\eta$ such that

$$
\int_{I_{(Y, \eta)}(z)} \eta=z^{3}
$$

Define $\Phi: U \times \Delta_{\epsilon^{2}} \rightarrow \Omega \mathcal{T}_{2}$ by

$$
\Phi((Y, \eta), z)=(Y, \eta) \#_{I_{(Y, \eta)}\left(z^{1 / 2}\right)} .
$$

$\Phi$ gives a conformal isomorphism of $\{(Y, \eta)\} \times \Delta_{\epsilon}$ onto a neighborhood of $(Y, \eta)$ in the leaf of $\mathcal{A}$ through $(Y, \eta)$. Since $\mathcal{A}$ is transverse to $\Omega \mathcal{T}_{2}(2)$, it follows that $\Phi$ is biholomorphic onto its image in $\Omega \mathcal{T}_{2}(2)$ if $U$ and $\epsilon$ are sufficiently small. By considering the inverse of $\Phi$, we obtain the following local coordinates around points in $\Omega \mathcal{T}_{2}(2)$. 
Proposition 6.1 Every $(X, \omega) \in \Omega \mathcal{T}(2)$ has a neighborhood $V$ such that:

- Each $(Y, \eta) \in V$ has either a shortest saddle connection I connecting distinct zeros or a double zero.

- The multi-valued function,

$$
(Y, \eta) \mapsto\left(\int_{I} \eta\right)^{2 / 3}
$$

has a single-valued branch $y$.

- The product Per $\times y: V \rightarrow H^{1}\left(\Sigma_{2} ; \mathbb{C}\right) \times \mathbb{C}$ is biholomorphic onto its image.

Period coordinates for $\Omega \mathcal{M}_{2}\left(T_{0,1}\right)$ The stratum $\Omega \mathcal{M}_{2}\left(T_{0,1}\right)$ is a suborbifold of $\Omega \overline{\mathcal{M}}_{2}$ isomorphic to the symmetric square of $\Omega \mathcal{M}_{1,1}$. We give local coordinates for this stratum just as for $\Omega \mathcal{M}_{2}(2)$ using the connected sum construction. As above, let Per: $\Omega \mathcal{D}_{2}\left(T_{0,1}\right) \rightarrow H^{1}\left(\Sigma_{2} ; \mathbb{C}\right)$ be the natural period map.

Each $(X, \omega) \in \Omega \mathcal{T}_{2}\left(T_{0,1}\right)$ is a one point connected sum of two genus one differentials:

$$
(X, \omega)=\left(X_{1}, \omega_{1}\right) \#\left(X_{2}, \omega_{2}\right) .
$$

Given a small open neighborhood $U \subset \Omega \mathcal{T}_{2}\left(T_{0,1}\right)$ of $(X, \omega)$ and a sufficiently small $\epsilon>0$, we define a holomorphic map $\Phi: U \times \Delta_{\epsilon} \rightarrow \Omega \mathcal{D}_{2}\left(T_{0,1}\right)$ by

$$
\Phi((X, \omega), z)=\left(X_{1}, \omega_{1}\right) \#_{I\left(z^{1 / 2}\right)}\left(X_{2}, \omega_{2}\right),
$$

where $I(z) \subset \mathbb{C}$ is the segment joining 0 to $\epsilon$.

If $(Y, \eta) \in \Omega \mathcal{D}_{2}\left(T_{0,1}\right)$ has a unique shortest saddle connection $I$, then define

$$
y(Y, \eta)=\left(\int_{I} \eta\right)^{2} .
$$

By taking an inverse of $\Phi$, we obtain local coordinates around $\Omega \mathcal{T}_{2}\left(T_{0,1}\right)$ as above.

Proposition 6.2 Every $(X, \omega) \in \Omega \mathcal{T}_{2}\left(T_{0,1}\right)$ has a neighborhood $V \subset \Omega \mathcal{D}_{2}\left(T_{0,1}\right)$ such that:

- Each $(Y, \eta) \in V$ has either a shortest saddle connection I connecting distinct zeros (up to the hyperelliptic involution) or a separating node.

- The function Per $\times y: V \rightarrow H^{1}\left(\Sigma_{2} ; \mathbb{C}\right) \times \mathbb{C}$ is biholomorphic onto its image. 


\subsection{The strata $\Omega \mathcal{M}_{2}^{0}\left(T_{1,0} ; 1,1\right)$ and $\Omega \mathcal{M}_{2}^{0}\left(T_{1,0} ; 2\right)$}

The locus $\Omega \mathcal{M}_{2}^{0}\left(T_{1,0}\right)$ - the union of the strata $\Omega \mathcal{M}_{2}^{0}\left(T_{1,0} ; 1,1\right)$ and $\Omega \mathcal{M}_{2}^{0}\left(T_{1,0} ; 2\right)$ - is a four dimensional orbifold. It can be naturally embedded in the bundle $\Omega^{\prime} \mathcal{M}_{1,2}$ consisting of meromorphic Abelian differentials $(X, \omega)$ on an elliptic curve $X$ with simple poles at two marked points $p$ and $q$ such that

$$
\operatorname{Res}_{p} \omega=-\operatorname{Res}_{q} \omega .
$$

Its image is the complement of the sub-line-bundle where these residues are zero. The stratum $\Omega \mathcal{M}_{2}^{0}\left(T_{1,0} ; 1,1\right)$ is an open, dense suborbifold of $\Omega \mathcal{M}_{2}^{0}\left(T_{1,0}\right)$, and $\Omega \mathcal{M}_{2}^{0}\left(T_{1,0} ; 2\right)$ is a closed, three-dimensional suborbifold.

Local coordinates We now give local coordinates on neighborhoods of these strata. Choose a symplectic basis $\left\{\alpha_{i}, \beta_{i}\right\}_{i=1}^{2}$ of $H_{1}\left(\Sigma_{2} ; \mathbb{Z}\right)$ such that $\alpha_{1}$ represents the single curve in $T_{1,0}$.

Define functions on $\Omega \mathcal{D}_{2}^{0}\left(T_{1,0}\right)$ as follows: given $(Y, \eta) \in \Omega \mathcal{D}_{2}^{0}\left(T_{1,0}\right)$, define

$$
\begin{aligned}
v & =\eta\left(\alpha_{1}\right) & w & =\eta\left(\alpha_{2}\right) \\
x & =\eta\left(\beta_{2}\right) & z & =e^{2 \pi i \eta\left(\beta_{1}\right) / \eta\left(\alpha_{1}\right)},
\end{aligned}
$$

where we consider $z(Y, \eta)$ to be 0 if $Y$ has a node. These are well-defined functions because $\alpha_{1}, \alpha_{2}$, and $\beta_{2}$ give well-defined homology classes on $Y$ via the marking, and $\beta_{1}$ gives a homology class on $Y$ which is well-defined up to adding a multiple of $\alpha_{1}$. These are all holomorphic functions on $U$. This follows from the fact that these functions are all holomorphic on $\Omega \mathcal{D}_{2}^{0}\left(T_{1,0}\right) \backslash \Omega \mathcal{T}_{2}^{0}\left(T_{1,0}\right)$ because periods vary holomorphically, together with the fact that these functions are all continuous on $U$, which follows from Theorem 5.5. Functions which are continuous and holomorphic on an open dense subset of their domain are holomorphic, so these functions $v, w, x$, and $z$ are all holomorphic.

Now, let $(X, \omega) \in \Omega \mathcal{T}_{2}^{0}\left(T_{1,0}\right)$, and let $U \subset \Omega \mathcal{D}_{2}^{0}\left(T_{1,0}\right)$ be an open neighborhood of $(X, \omega)$ with a continuous choice of a saddle connection $I_{(Y, \eta)}$ on each $(Y, \eta) \in U$ joining distinct zeros. If $(X, \omega)$ has two simple zeros, then we can also continuously orient these saddle connections, but this is not possible if $(X, \omega)$ has a double zero. For $(Y, \eta) \in U$, define

$$
y= \begin{cases}\int_{I_{(Y, \eta)}} \eta & \text { if }(X, \omega) \text { has two simple zeros } \\ \left(\int_{I_{(Y, \eta)}} \eta\right)^{2 / 3} & \text { if }(X, \omega) \text { has a double zero }\end{cases}
$$


If $U$ is sufficiently small, then the functions $(v, w, x, y)$ are a system of holomorphic local coordinates on $\Omega \mathcal{T}_{2}^{0}\left(T_{1,0}\right) \cap U$ by (a simple generalization of) the period coordinates from [78] together with the argument of Proposition 6.1.

Recall that in Section 4.1, we defined the unplumbing operation which replaced an Abelian differential with a choice of a cylinder with a new meromorphic Abelian differential with two simple poles. Since these poles have opposite residues, we can join them to a node and obtain a stable Abelian differential.

Define the unplumbing map to be the function,

$$
\psi: \Omega \mathcal{D}_{2}^{0}\left(T_{1,0}\right) \rightarrow \Omega \mathcal{T}_{2}^{0}\left(T_{1,0}\right),
$$

defined by sending $(Y, \eta)$ to the Abelian differential obtained by unplumbing the cylinder on $Y$ which is homotopic to the single curve in the curve system $T_{1,0}$ (we define $\phi$ to be the identity on $\Omega \mathcal{T}_{2}^{0}\left(T_{1,0}\right)$. The function $\psi$ is continuous and is holomorphic on

$$
\Omega \mathcal{D}_{2}^{0}\left(T_{1,0}\right) \backslash \Omega \mathcal{T}_{2}^{0}\left(T_{1,0}\right)
$$

as can easily be seen using period coordinates, so $\psi$ is holomorphic on all of $\Omega \mathcal{D}_{2}^{0}\left(T_{1,0}\right)$. Let $\Psi=\psi \times z$.

Proposition 6.3 The map $\Psi$ is a biholomorphic map of $\Omega \mathcal{D}_{2}^{0}\left(T_{1,0}\right)$ onto its image in $\Omega \mathcal{T}_{2}^{0}\left(T_{1,0}\right) \times \mathbb{C}$.

It follows that if $U$ is sufficiently small, the functions $(v, w, x, y, z)$ are a holomorphic system of local coordinates on the neighborhood $U$ of $(X, \omega)$.

Proof We can show that $\Psi$ is biholomorphic onto its image by constructing a holomorphic inverse. Given $(Y, \eta) \in \Omega \mathcal{T}_{2}^{0}\left(T_{1,0}\right)$ and $u \in \mathbb{C}^{*}$, if $|u|$ is sufficiently small, then we can uniquely plumb a cylinder into the node of $(Y, \eta)$ to form a new nonsingular Abelian differential $\left(Y^{\prime}, \eta^{\prime}\right)$ such that $z\left(Y^{\prime}, \eta^{\prime}\right)=u$. The point is that the plumbing construction is determined by two quantities: the height of the resulting cylinder, and the twist of the gluing map; the height is determined by $|u|$, and the twist is determined by $\arg u$.

Let $V \subset \Omega \mathcal{T}_{2}^{0}\left(T_{1,0}\right) \times \mathbb{C}$ be the open neighborhood of $\Omega \mathcal{T}_{2}^{0}\left(T_{1,0}\right) \times\{0\}$ where this operation is defined, and let $\Phi: V \rightarrow \Omega \mathcal{D}_{2}^{0}\left(T_{1,0}\right)$ be the plumbing map. The map $\Phi$ is continuous and is holomorphic on $V \backslash\left(\Omega \mathcal{T}_{2}^{0}\left(T_{1,0}\right) \times\{0\}\right)$ by period coordinates, so it is everywhere holomorphic. The maps $\Phi$ and $\Psi$ are inverse to each other, so both are biholomorphic.

The last statement then follows from the fact that $(v, w, x, y)$ are a system of local coordinates on a neighborhood of $(X, \omega)$ in $\Omega \mathcal{T}_{2}^{0}\left(T_{1,0}\right)$. 
Cylinder covers The stratum $\mathcal{M}_{2}\left(T_{1,0}\right) \subset \overline{\mathcal{M}}_{2}$ of stable Riemann surfaces with one nonseparating node is naturally isomorphic to the moduli space $\mathcal{M}_{1,2}$ of elliptic curves with two marked points, and we will implicitly identify them. In $\mathcal{M}_{2}\left(T_{1,0}\right)$, let $\mathcal{M}_{2}\left(T_{1,0}\right)(d)$ be the locus of all $X$ such that the two marked points on $X$ differ by exactly $d$-torsion in the group law on the underlying elliptic curve. This locus $\mathcal{M}_{2}\left(T_{1,0}\right)(d)$ is isomorphic to modular curve $\mathbb{H} / \Gamma_{1}(d)$.

Given a marked elliptic curve $X$ in $\mathcal{M}_{1,2}$, call a meromorphic function $f: X \rightarrow \mathbb{P}^{1}$ a cylinder cover if all of the poles and zeros of $f$ are located at the two marked points of $X$. By Riemann-Hurwitz, such an $f$ has two other branch points counting multiplicity. We say that $f$ is primitive if $f$ is not of the form $g^{r}$ for some meromorphic function $g$ and $r>1$. A meromorphic Abelian differential $(X, \omega)$ in $\Omega^{\prime} \mathcal{M}_{1,2}$ is a cylinder covering differential if $\omega=d f / f$ for some primitive cylinder cover (in which case $\omega=f^{*}(d z / z)$, the pullback of the form which makes $\mathbb{C}^{*}$ a flat infinite cylinder). The degree of $\omega$ is the degree of the primitive cover. In terms of stable Riemann surfaces, a cylinder cover is a morphism $f: X \rightarrow C$, where $X \in \mathcal{M}_{2}\left(T_{1,0}\right)$, the curve $C$ is $\mathbb{P}^{1}$ with 0 and $\infty$ identified to form a node, and the inverse image of the node of $C$ is the node of $X$.

Denote by $\Omega \mathcal{M}_{2}^{0}\left(T_{1,0}\right)(d)$ the locus of degree $d$ cylinder covering differentials in $\Omega \mathcal{M}_{2}^{0}\left(T_{1,0}\right)$.

Proposition 6.4 A stable Riemann surface $X \in \mathcal{M}_{2}\left(T_{1,0}\right)$ has a degree $d$ cylinder covering differential if and only if $X \in \mathcal{M}_{2}\left(T_{1,0}\right)(d)$, in which case it unique up to constant multiple.

Proof To prove uniqueness, assume that $f$ and $g$ are two degree $d$ cylinder covers. This means that $f$ and $g$ have the same zeros and poles, so $f=c g$ for some $c \in \mathbb{C}$. Thus $d f / f=d g / g$.

Recall that if $E$ is an elliptic curve and $\operatorname{Div}^{0}(E)$ is the group of divisors of degree 0 on $E$, then there is a natural map $\mu$ : $\operatorname{Div}^{0}(E) \rightarrow E$, defined via the group law on $E$. Abel's Theorem says that a divisor $D \in \operatorname{Div}^{0}(E)$ is the divisor of a meromorphic function on $E$ if and only if $\mu(D)=0$ (see Griffiths and Harris [27, p 235]).

Now let $X \in \mathcal{M}_{2}\left(T_{1,0}\right)$, which we'll regard as an elliptic curve with two marked points $p$ and $q$. Choose an identity point 0 on $X$, fixing the group law. If $X$ has a degree $d$ cylinder cover $f: X \rightarrow \mathbb{P}^{1}$, with its zeros at $p$ and its poles at $q$, then $(f)=d(p-q)$ (where $(f)$ denotes the divisor associated to $f$ ), and by Abel's Theorem, $d(p-q)=0$ in the group law on $E$; that is, $p$ and $q$ differ by $d$-torsion.

We need to show that there is no $e<d$ with $e \mid d$ and $e(p-q)=0$. Suppose there is such an $e$. Then by Abel's Theorem again, there would be a meromorphic function $h$ 
on $X$ with $(h)=e(p-q)$, which implies that $h$ is a degree $e$ cylinder cover. By the uniqueness statement, $f=c h^{d / e}$ for some $c \in \mathbb{C}$, contradicting primitivity of $f$.

The proof of the converse of this proposition is also a straightforward application of Abel's Theorem and will be left to the reader.

Corollary 6.5 The suborbifold $\mathbb{P} \Omega \mathcal{M}_{2}^{0}\left(T_{1,0}\right)(d)$ of $\mathbb{P} \Omega \mathcal{M}_{2}^{0}\left(T_{1,0}\right)$ is isomorphic to $\mathbb{U} / \Gamma_{1}(d)$.

\subsection{The stratum $\Omega \mathcal{M}_{2}^{0}\left(T_{1,0}, T_{1,0}^{1}\right)$}

We now give local coordinates around the stratum $\Omega \mathcal{M}_{2}^{0}\left(T_{1,0}, T_{1,0}^{1}\right)$. We will actually only give local coordinates on a hypersurface containing this stratum, but this will be sufficient for our purposes.

Let $\left\{\alpha_{i}, \beta_{i}\right\}_{i=1}^{2}$ be a symplectic basis of $H_{1}\left(\Sigma_{2} ; \mathbb{Z}\right)$ as in the previous section. Given $(Y, \eta) \in \Omega \mathcal{D}_{2}^{0}\left(T_{1,0}, T_{1,0}^{1}\right)$, define

$$
\begin{array}{ll}
w=\eta\left(\alpha_{2}\right) & x=\eta\left(\beta_{2}\right) \\
y=\eta\left(\beta_{1}\right) & z=\left(\int_{I} \eta\right)^{2},
\end{array}
$$

where $I$ is one of the two saddle connections on $(Y, \eta)$ such that $I \cup J(I)$ is homotopic to the curve $T_{1,0}^{1}$ in $T_{1,0}$. These are holomorphic functions on $\Omega \mathcal{D}_{2}^{0}\left(T_{1,0}, T_{1,0}^{1}\right)$. If $(Y, \eta) \in \Omega \mathcal{T}_{2}^{0}\left(T_{1,0}, T_{1,0}^{1}\right)$ is regarded as a genus one differential with two marked points, then $y(Y, \eta)$ is the integral of $\eta$ along a path joining the marked points. The functions $(w, x, y)$ are a system of holomorphic local coordinates on $\Omega \mathcal{T}_{2}^{0}\left(T_{1,0}, T_{1,0}^{1}\right)$.

Proposition 6.6 For any $(X, \omega) \in \Omega \mathcal{T}_{2}^{0}\left(T_{1,0}, T_{1,0}^{1}\right)$, the functions $(w, x, y, z)$ define a system of holomorphic local coordinates on a sufficiently small neighborhood of $(X, \omega)$ in $\Omega \mathcal{D}_{2}^{0}\left(T_{1,0}, T_{1,0}^{1}\right)$.

Sketch of proof The proof of this proposition is completely analogous to the proof of Proposition 6.3. Let

$$
\psi: \Omega \mathcal{D}_{2}^{0}\left(T_{1,0}, T_{1,0}^{1}\right) \rightarrow \Omega \mathcal{T}_{2}^{0}\left(T_{1,0}, T_{1,0}^{1}\right)
$$

be the map which sends an Abelian differential to the one obtained by splitting along the union of saddle connections $I \cup J(I)$ homotopic to the curve $T_{1,0}^{1}$. Let $\Psi=\psi \times z$. This is a holomorphic map

$$
\Psi: \Omega \mathcal{D}_{2}^{0}\left(T_{1,0}, T_{1,0}^{1}\right) \rightarrow \Omega \mathcal{T}_{2}^{0}\left(T_{1,0}, T_{1,0}^{1}\right) \times \mathbb{C} .
$$


Given any $u \in \mathbb{C}$, let $I(u) \subset \mathbb{C}$ be the segment connecting 0 to $u$. For any $(Y, \eta) \in$ $\Omega \mathcal{T}_{2}^{0}\left(T_{1,0}, T_{1,0}^{1}\right)$, regarded as a genus one differential with two marked points, if $|u|$ is sufficiently small, then there are two parallel embeddings

$$
\epsilon_{i}: I\left(u^{1 / 2}\right) \rightarrow(Y, \eta)
$$

each sending 0 to one of the marked points of $(Y, \eta)$. Let

$$
V \subset \Omega \mathcal{T}_{2}^{0}\left(T_{1,0}, T_{1,0}^{1}\right) \times \mathbb{C}
$$

be the neighborhood of $\Omega \mathcal{T}_{2}^{0}\left(T_{1,0}, T_{1,0}^{1}\right) \times\{0\}$ where these two embeddings $\epsilon_{i}$ exist, and define

$$
\Phi: V \rightarrow \Omega \mathcal{D}_{2}^{0}\left(T_{1,0}, T_{1,0}^{1}\right)
$$

to be the map which replaces $(Y, \eta)$ with the self connected sum

$$
(Y, \eta) \#_{I\left(z^{1 / 2}\right)} .
$$

$\Phi$ is a holomorphic map, and since $\Phi$ and $\Psi$ are inverse to each other, both are biholomorphic. Since $(w, x, y)$ give local coordinates on $\Omega \mathcal{T}_{2}^{0}\left(T_{1,0}, T_{1,0}^{1}\right)$ around $(X, \omega)$, it then follows that $(w, x, y, z)$ give local coordinates on $\Omega \mathcal{D}_{2}^{0}\left(T_{1,0}, T_{1,0}^{1}\right)$ around $(X, \omega)$.

\section{The d-torsion locus Let}

$$
\mathbb{P} \Omega \mathcal{M}_{2}^{0}\left(T_{1,0}, T_{1,0}^{1}\right)(d) \subset \mathbb{P} \Omega \mathcal{M}_{2}^{0}\left(T_{1,0}, T_{1,0}^{1}\right)
$$

be the locus of genus one differentials such that the two marked points differ by torsion of degree exactly $d$. One can show using period coordinates that $\mathbb{P} \Omega \mathcal{M}_{2}^{0}\left(T_{1,0}, T_{1,0}^{1}\right)(d)$ is a suborbifold of $\mathbb{P} \Omega \mathcal{M}_{2}^{0}\left(T_{1,0}, T_{1,0}^{1}\right)$. We have the isomorphism

$$
\mathbb{P} \Omega \mathcal{M}_{2}^{0}\left(T_{1,0}, T_{1,0}^{1}\right)(d) \cong \mathcal{M}_{2}\left(T_{1,0}\right)(d) \cong \mathbb{H} / \Gamma_{1}(d) .
$$

\subsection{The stratum $\Omega \mathcal{M}_{2}^{0}\left(T_{2,0}, T_{2,0}^{2}\right)$}

We now consider the stratum $\Omega \mathcal{M}_{2}^{0}\left(T_{2,0}, T_{2,0}^{2}\right)$, and define local coordinates on a hypersurface containing this stratum. Choose a symplectic basis $\left\{\alpha_{i}, \beta_{i}\right\}_{i=1}^{2}$ of $H_{1}\left(\Sigma_{2} ; \mathbb{Z}\right)$ such that $\alpha_{i}$ represents the curve $T_{2,0}^{i}$ in $T_{2,0}$. Given $(Y, \eta) \in \Omega \mathcal{D}_{2}^{0}\left(T_{2,0}, T_{2,0}^{2}\right)$, define

$$
\begin{array}{ll}
w=\eta\left(\alpha_{1}\right) & x=\eta\left(\beta_{2}\right) \\
y=e^{2 \pi i \eta\left(\beta_{1}\right) / \eta\left(\alpha_{1}\right)} & z=\left(\int_{I} \eta\right)^{2},
\end{array}
$$


where $I$ is a saddle connection joining distinct zeros on $(Y, \eta)$ such that $I \cup J(I)$ is homotopic to the curve $T_{2,0}^{2}$. These are holomorphic functions on $\Omega \mathcal{D}_{2}^{0}\left(T_{2,0}, T_{2,0}^{2}\right)$, and the functions $(w, x)$ are holomorphic local coordinates on $\Omega \mathcal{T}_{2}^{0}\left(T_{2,0}, T_{2,0}^{2}\right)$.

Proposition 6.7 Given $(X, \omega) \in \Omega \mathcal{T}_{2}^{0}\left(T_{2,0}, T_{2,0}^{2}\right)$, the functions $(w, x, y, z)$ give a system of holomorphic local coordinates on a sufficiently small neighborhood $U$ of $(X, \omega)$ in $\Omega \mathcal{D}_{2}^{0}\left(T_{2,0}, T_{2,0}^{2}\right)$.

The proof is a straightforward combination of the proofs of Propositions 6.3 and 6.6, so we will omit it as well of the proofs of further similar descriptions of local coordinates.

Points in $\mathbb{P} \boldsymbol{\Omega} \mathcal{M}_{\mathbf{2}}^{\mathbf{0}}\left(\boldsymbol{T}_{\mathbf{2}, \mathbf{0}}, \boldsymbol{T}_{\mathbf{2}, \mathbf{0}}^{\mathbf{2}}\right)$ Every Abelian differential in $\mathbb{P} \Omega \mathcal{M}_{2}^{0}\left(T_{2,0}, T_{2,0}^{2}\right)$ can be obtained by the following construction: given $r \in \mathbb{C} / \mathbb{Z}$, let $f_{r}$ be the Abelian differential obtained from the cylinder $(\mathbb{C} / \mathbb{Z}, d z)$ by identifying the ends of the cylinder to form a polar node and identifying the points 0 and $r$ to form a holomorphic node. Two Abelian differentials $f_{r}$ and $f_{r^{\prime}}$ are the same if and only if $r$ and $r^{\prime}$ are related by the group $G$ generated by the transformations $z \mapsto z+1$ and $z \mapsto-z$. This gives an isomorphism of $\mathbb{P} \Omega \mathcal{M}_{2}^{0}\left(T_{2,0}, T_{2,0}^{2}\right)$ with

$$
(\mathbb{C} \backslash \mathbb{Z}) / G \cong(\mathbb{C} \backslash\{0,1\}) /\left(z \mapsto z^{-1}\right) .
$$

In $\mathbb{P} \Omega \mathcal{M}_{2}^{0}\left(T_{2,0}, T_{2,0}^{2}\right)$, let

$$
\mathbb{P} \Omega \mathcal{M}_{2}^{0}\left(T_{2,0}, T_{2,0}^{2}\right)(d)=\bigcup_{q \in \Lambda} f_{q / d}
$$

where $\quad \Lambda=\{q \in \mathbb{Z}: \operatorname{gcd}(q, d)=1\}$.

We can regard $\mathbb{P} \Omega \mathcal{M}_{2}^{0}\left(T_{2,0}, T_{2,0}^{2}\right)(d)$ as the locus of stable Abelian differentials $(X,[\omega]) \in \mathbb{P} \Omega \mathcal{M}_{2}^{0}\left(T_{2,0}, T_{2,0}^{2}\right)$ such that - if we regard $(X, \omega)$ as $\left(\mathbb{C}^{*}, d z / z\right)$ with two marked points - the two marked points differ by exactly $d$-torsion in $\mathbb{C}^{*}$.

$\mathbb{P} \Omega \mathcal{M}_{2}^{0}\left(T_{2,0}, T_{2,0}^{2}\right)(d)$ contains $N$ points, where

$$
N= \begin{cases}1 & \text { if } d=2 \\ \frac{1}{2} \phi(d) & \text { if } d>2\end{cases}
$$

\subsection{The strata $\Omega \mathcal{M}_{2}^{0}\left(T_{2,0} ; 1,1\right), \Omega \mathcal{M}_{2}^{0}\left(T_{2,0} ; 2\right)$, and $\Omega \mathcal{M}_{2}^{0}\left(T_{2,1}\right)$}

We now give local coordinates around the strata $\Omega \mathcal{M}_{2}^{0}\left(T_{2,0} ; 1,1\right), \Omega \mathcal{M}_{2}^{0}\left(T_{2,0} ; 2\right)$, and $\Omega \mathcal{M}_{2}^{0}\left(T_{2,1}\right)$. In each of these cases, let $\left\{\alpha_{i}, \beta_{i}\right\}_{i=1}^{2}$ be a symplectic basis of $H_{1}\left(\Sigma_{2} ; \mathbb{Z}\right)$ such that the $\alpha_{i}$ represent the nonseparating curves of the curve system. 
Fix an Abelian differential $(X, \omega)$ in $\Omega \mathcal{T}_{2}^{0}\left(T_{2,0} ; 1,1\right), \Omega \mathcal{T}_{2}^{0}\left(T_{2,0} ; 2\right)$, or $\Omega \mathcal{T}_{2}^{0}\left(T_{2,1}\right)$, and choose a small neighborhood $U$ of $(X, \omega)$ in $\Omega \mathcal{D}_{2}^{0}\left(T_{2,0} ; 1,1\right), \Omega \mathcal{D}_{2}^{0}\left(T_{2,0}\right)$, or $\Omega \mathcal{D}_{2}^{0}\left(T_{2,1}\right)$ respectively. If $U$ is sufficiently small, then we can continuously choose for each $(Y, \eta) \in U$ having distinct zeros a saddle connection $I_{(Y, \eta)}$ connecting those zeros. If $(X, \omega)$ has two distinct zeros, then we can also continuously choose an orientation for these saddle connections, but this is not possible if it has a double zero or a separating node. Given $(Y, \eta) \in U$, define

$$
\begin{gathered}
v=\eta\left(\alpha_{1}\right) \\
y=e^{2 \pi i \eta\left(\beta_{1}\right) / \eta\left(\alpha_{1}\right)} \\
z=e^{2 \pi i \eta\left(\beta_{2}\right) / \eta\left(\alpha_{2}\right)} \\
x= \begin{cases}\int_{I} \eta & \text { if }(X, \omega) \in \Omega \mathcal{T}_{2}^{0}\left(T_{2,0} ; 1,1\right) ; \\
\left(\int_{I} \eta\right)^{2 / 3} & \text { if }(X, \omega) \in \Omega \mathcal{T}_{2}^{0}\left(T_{2,0} ; 2\right) ; \\
\left(\int_{I} \eta\right)^{2} & \text { if }(X, \omega) \in \Omega \mathcal{T}_{2}^{0}\left(T_{2,1}\right) .\end{cases}
\end{gathered}
$$

These are holomorphic functions on $U$, and $(v, w, x)$ give a holomorphic system of local coordinates on $\Omega \mathcal{T}_{2}^{0}\left(T_{2,0}\right)$ in the first two cases. In the last case where $(X, \omega) \in \Omega \mathcal{T}_{2}^{0}\left(T_{2,1}\right)$, the $(v, w, x)$ give a system of local coordinates near $(X, \omega)$ on the subspace of $\Omega \mathcal{D}_{2}^{0}\left(T_{2,1}\right)$ where the nonseparating curves of $T_{2,1}$ remain nodes.

Proposition 6.8 The functions $(v, w, x, y, z)$ give a system of holomorphic local coordinates on a neighborhood $U$ of $(X, \omega)$ in $\Omega \mathcal{D}_{2}^{0}\left(T_{2,0} ; 1,1\right), \Omega \mathcal{D}_{2}^{0}\left(T_{2,0} ; 2\right)$, or $\Omega \mathcal{D}_{2}^{0}\left(T_{2,1}\right)$.

Curves in $\mathbb{P} \boldsymbol{\Omega} \overline{\mathcal{M}}_{\mathbf{2}}$ We now discuss some curves in $\mathbb{P} \Omega \overline{\mathcal{M}}_{2}$ which will play a prominent role in the rest of this paper. Given $\lambda \in \mathbb{C}^{*}$, let $C_{\lambda}$ be the locus of points $(X,[\omega]) \in \mathbb{P} \Omega \overline{\mathcal{M}}_{2}$ having two nonseparating nodes and possibly a separating node such that the ratio of the residues of $\omega$ at the two nonseparating nodes of $X$ is $\pm \lambda^{ \pm 1}$. Two such curves $C_{\lambda}$ and $C_{\lambda^{\prime}}$ coincide if and only if $\lambda^{\prime}= \pm \lambda^{ \pm 1}$. Also, let $c_{\lambda} \in \mathbb{P} \Omega \overline{\mathcal{M}}_{2}$ be the Abelian differential with three nonseparating nodes and residues $1, \lambda$, and $\lambda-1$ as in Figure 8.

Proposition 6.9 The curve $C_{\lambda} \subset \mathbb{P} \Omega \overline{\mathcal{M}}_{2}$ is a twice-punctured sphere. If $\lambda= \pm 1$, then it is an orbifold locus of order two; otherwise it contains no orbifold points of $\mathbb{P} \Omega \overline{\mathcal{M}}_{2}$. The punctures of $C_{\lambda}$ are the two points $c_{\lambda}$ and $c_{\lambda+1}$. The curve $C_{\lambda}$ contains exactly one point of $\mathbb{P} \Omega \mathcal{M}_{2}^{0}\left(T_{2,1}\right)$.

Proof Let $C_{\lambda}^{0} \subset C_{\lambda}$ be the subset of differentials with no separating node. We claim that $C_{\lambda}^{0}$ is a thrice-punctured sphere. The natural map $C_{\lambda}^{0} \rightarrow \mathcal{M}_{2}\left(T_{2,0}\right)$ is an 


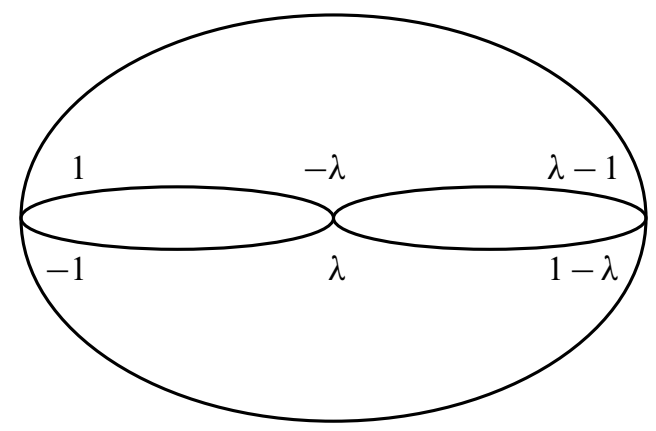

Figure 8: The point $c_{\lambda} \in \mathbb{P} \Omega \overline{\mathcal{M}}_{2}$

isomorphism. Let $\mathcal{M}_{0,4}^{\text {num }}$ be the moduli space of four numbered points on $\mathbb{P}^{1}$. There is a natural map $\pi: \mathcal{M}_{0,4}^{\text {num }} \rightarrow \mathcal{M}_{2}\left(T_{2,0}\right)$ which sends the point $\left(p_{1}, p_{2}, p_{3}, p_{4}\right) \in \mathcal{M}_{0,4}^{\text {num }}$ to the stable Riemann surface obtained by identifying $\left(p_{1}, p_{2}\right)$ and $\left(p_{3}, p_{4}\right)$ to nodes. There are four ways to renumber the points $p_{i}$ to get the same stable Riemann surface, so a fiber of $\pi$ potentially contains four points; however, the automorphism group of the $p_{i}$ contains a Klein four-group whose action on the $p_{i}$ realizes any renumbering of the $p_{i}$ which preserves the decomposition into the two sets $\left\{p_{1}, p_{2}\right\}$ and $\left\{p_{3}, p_{4}\right\}$. Therefore $\pi$ is an isomorphism. There is thus an isomorphism $\tilde{\pi}: \mathcal{M}_{0,4}^{\text {num }} \rightarrow C_{\lambda}^{0}$, defined by sending the point $Q=\left(p_{1}, p_{2}, p_{3}, p_{4}\right) \in \mathcal{M}_{0,4}^{\text {num }}$ to $(X, \omega)$, where $X=\pi(Q)$, and $\omega$ is induced by the unique meromorphic Abelian differential $\eta$ on $\mathbb{P}^{1}$ having a simple pole at each $p_{i}$ with residue given by

$$
\begin{array}{ll}
\operatorname{Res}_{p_{1}} \eta=1 & \operatorname{Res}_{p_{2}} \eta=-1 \\
\operatorname{Res}_{p_{3}} \eta=\lambda & \operatorname{Res}_{p_{4}} \eta=-\lambda .
\end{array}
$$

It is well known that $\mathcal{M}_{0,4}^{\text {num }}$ is a thrice-punctured sphere, so $C_{\lambda}^{0}$ is as well.

Cusps of $\mathcal{M}_{0,4}^{\text {num }}$ correspond to isotopy classes of simple closed curves in $\mathbb{P}^{1} \backslash\left\{p_{i}\right\}_{i=1}^{4}$ up to the action of the modular group $\operatorname{Mod}_{0,4}^{\text {num }}$ of self-homeomorphisms of $\mathbb{P}^{1}$ fixing each of the $p_{i}$ up to isotopy fixing the $p_{i}$. There are three such isotopy classes of curve. These are shown in Figure 9 with the corresponding limiting Abelian differential in $\mathbb{P} \Omega \overline{\mathcal{M}}_{2}$. Thus we see that $C_{\lambda}$ contains one point in $\mathbb{P} \Omega \mathcal{M}_{2}^{0}\left(T_{2,1}\right)$, and the two cusps of $C_{\lambda}$ are as claimed.

To obtain the statement about orbifold points, note that for each $(X,[\omega]) \in C_{\lambda}$, the automorphism group of $X$ is the Klein four group. If $\lambda= \pm 1$, then all of these automorphisms stabilize the projective class of $\omega$, while if $\lambda \neq \pm 1$, only the hyperelliptic involution stabilizes $[\omega]$. 

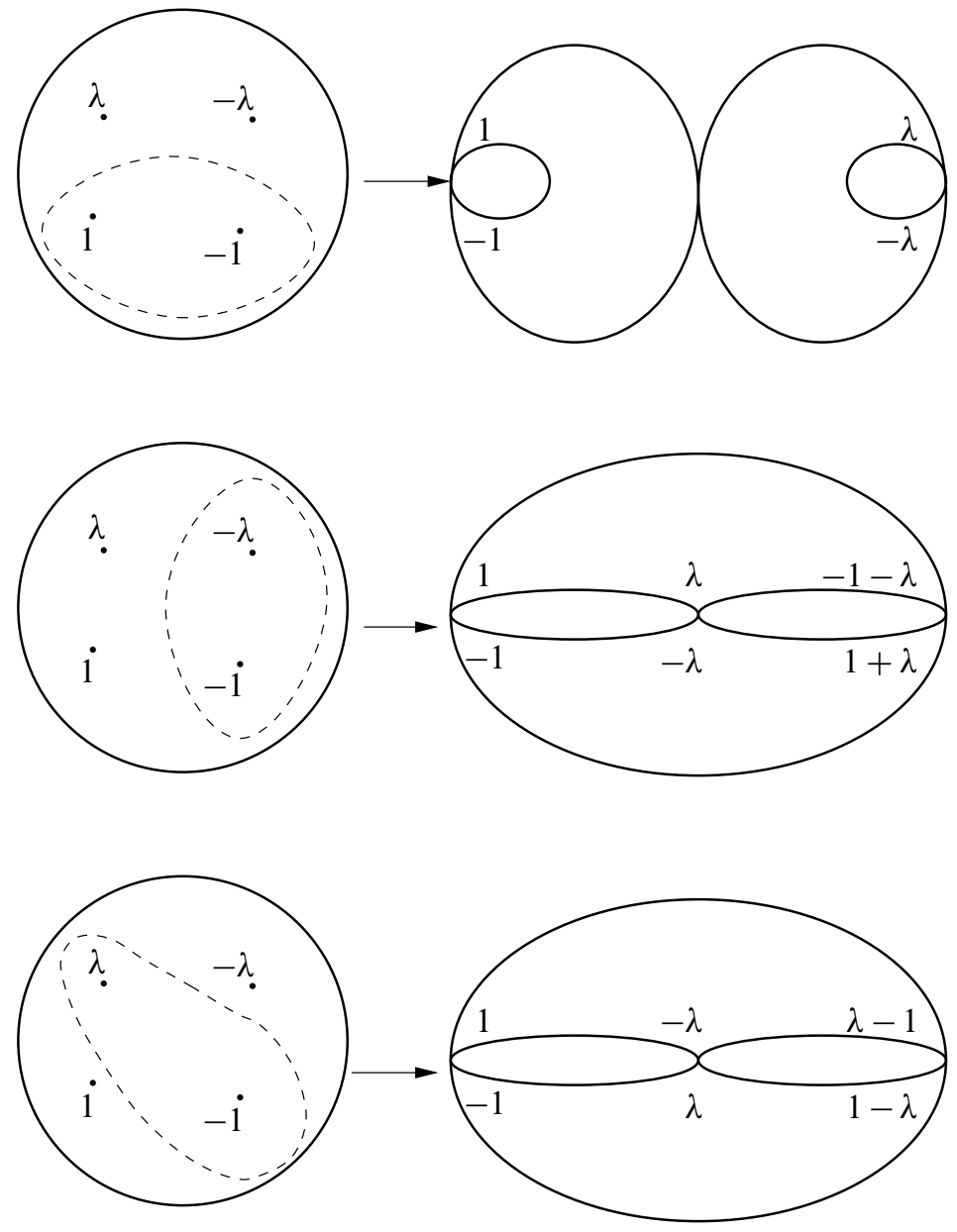

Figure 9: Three curves determining cusps of $C_{\lambda}^{0}$

Proposition 6.10 If $\lambda \neq \pm 1$, then $\mathbb{P} \Omega \mathcal{M}_{2}^{0}\left(T_{2,0} ; 2\right)$ meets $C_{\lambda}$ in exactly one point. Otherwise they are disjoint.

Proof Define an isomorphism $\pi: \mathbb{C} \backslash\{0,1\} \rightarrow C_{\lambda}^{0}$ by $p(w)=\left(X_{w}, \omega_{w}\right)$, where $X_{w}$ is the stable Riemann surface obtained by identifying the points $\{0,1\}$ and $\{w, \infty\}$ to nodes, and $\omega_{w}$ is induced by the meromorphic Abelian differential on $\mathbb{P}^{1}$.,

$$
\begin{aligned}
\omega_{w}^{\prime} & =\left(\frac{1}{z}-\frac{1}{z-1}+\frac{\lambda}{z-w}\right) d z \\
& =\frac{\lambda z^{2}+(-1-\lambda) z+w}{z(z-1)(z-w)} d z,
\end{aligned}
$$


The differential $\omega_{w}^{\prime}$ has a double zero if and only if

$$
\lambda z^{2}+(-1-\lambda) z+w
$$

has a double zero in $\mathbb{C} \backslash\{0,1, w\}$, which happens if and only if the discriminant,

$$
\Delta_{w}=(1+\lambda)^{2}-4 \lambda w
$$

vanishes for some $w \in \mathbb{C} \backslash\{0,1\}$. This happens if and only if $\lambda \neq \pm 1$, in which case, there is a unique $w$ such that $\Delta_{w}=0$. Thus there is a unique Abelian differential in $C_{\lambda}$ with a double zero if $\lambda \neq \pm 1$, and there is no such Abelian differential if $\lambda= \pm 1$.

For $\lambda \neq 0$ or \pm 1 , let $w_{\lambda} \in C_{\lambda}$ be the unique point representing a stable Abelian differential with a double zero. Also, for $\lambda \neq 0$, let $p_{\lambda} \in C_{\lambda}$ be the unique point representing a stable Abelian differential with a nonseparating node.

\subsection{The stratum $\Omega \mathcal{M}_{2}^{0}\left(T_{3,0}\right)$}

We now give local coordinates around points in $\Omega \mathcal{M}_{2}^{0}\left(T_{3,0}\right)$, the stratum of stable Abelian differentials with three nonseparating polar nodes. There is a unique stable Riemann surface $X \in \mathcal{M}_{2}\left(T_{3,0}\right)$ consisting of two thrice-punctured spheres with the cusps joined together to form three separating nodes.

Choose two points $p, q \in \Sigma_{2} \backslash T_{3,0}$. Let $\alpha_{i} \in H_{1}\left(\Sigma_{2} \backslash\{p, q\} ; \mathbb{Z}\right)$ be a homology class representing the curve $T_{3,0}^{i} \in T_{3,0}$. Let $\gamma_{i} \in H_{1}\left(\Sigma_{2},\{p, q\} ; \mathbb{Z}\right)$ be homology classes such that $\alpha_{i} \cdot \gamma_{j}=\delta_{i j}$ (see Figure 10).

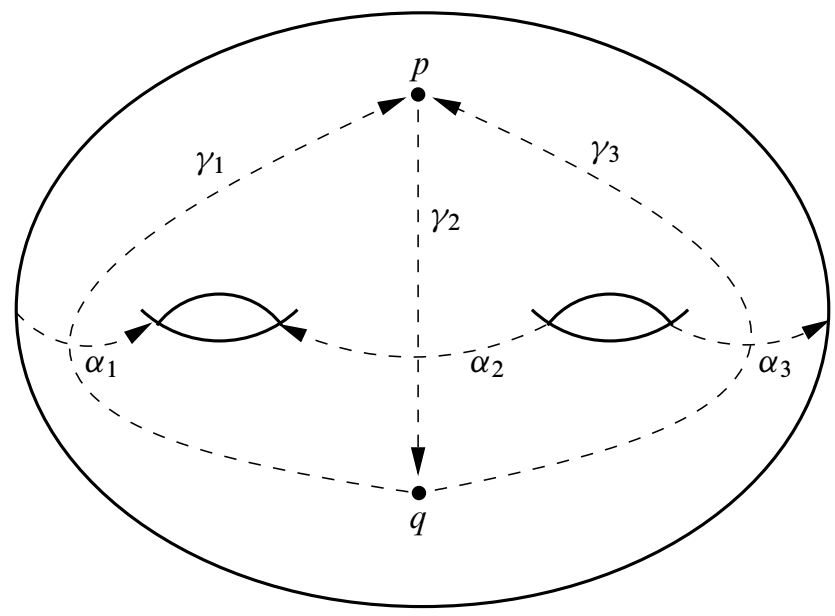

Figure 10: Homology classes in $H_{1}\left(\Sigma_{2},\{p, q\} ; \mathbb{Z}\right)$ 
Any marked stable Abelian differential $(f,(Y, \eta)) \in \Omega \mathcal{D}_{2}^{0}\left(T_{3,0}\right)$ has three cylinders $C_{i}$ homotopic to the curves $T_{3,0}^{i}$ of $T_{3,0}$ and two simple zeros, one in each component of the complement of the cylinders. We can change $f$ to a marking $f_{1}$ by an isotopy so that $f_{1}$ takes each curve $T_{3,0}^{i}$ into the cylinder $C_{i}$ and takes the points $p$ and $q$ to zeros of $\eta$. Two such markings $f_{1}$ and $f_{2}$ which are isotopic to $f$ are isotopic to each other by an isotopy sending for all time the points $p$ and $q$ to zeros of $\eta$ and sending the curve $T_{3,0}^{i}$ into the cylinder $C_{i}$. This means that we can regard the classes $\alpha_{i}$ as homology classes in $H_{1}(Y \backslash Z(\eta) ; \mathbb{Z})$ and the $\gamma_{i}$ as homology classes in $H_{1}(Y, Z(\eta) ; \mathbb{Z})$, well-defined up to adding a multiple of $\alpha_{i}$ (because the marking $f$ was only defined up to Dehn twist around the curves of $T_{3,0}$ ).

Given $(Y, \eta) \in \Omega \mathcal{D}_{2}^{0}\left(T_{3,0}\right)$, define:

$$
\begin{aligned}
& v=\eta\left(\alpha_{1}\right) \quad w=\eta\left(\alpha_{2}\right) \\
& x=e^{2 \pi i \eta\left(\gamma_{1}\right) / \eta\left(\alpha_{1}\right)} \quad y=e^{2 \pi i \eta\left(\gamma_{2}\right) / \eta\left(\alpha_{2}\right)} \\
& z=e^{2 \pi i \eta\left(\gamma_{3}\right) / \eta\left(\alpha_{3}\right)}
\end{aligned}
$$

These are well-defined holomorphic functions on $\Omega \mathcal{D}_{2}^{0}\left(T_{3,0}\right)$, and the coordinates $(v, w)$ define a map $\Omega \mathcal{T}_{2}^{0}\left(T_{3,0}\right) \rightarrow \mathbb{C}^{2}$ which is biholomorphic onto its image.

Proposition 6.11 The functions $(v, w, x, y, z)$ define a map $\Omega \mathcal{D}_{2}^{0}\left(T_{3,0}\right) \rightarrow \mathbb{C}^{5}$ which is biholomorphic onto its image.

\subsection{The stratum $\Omega \mathcal{M}_{2}^{0}\left(T_{3,0}, T_{3,0}^{3}\right)$}

Finally, consider the stratum $\Omega \mathcal{M}_{2}^{0}\left(T_{3,0}, T_{3,0}^{3}\right)$. An Abelian differential in this stratum can be regarded as a pair of infinite cylinders with an end of each cylinder identified with an end of the other cylinder to form two polar nodes, and with a point from each cylinder identified to form a holomorphic node. The stratum $\Omega \mathcal{M}_{2}^{0}\left(T_{3,0}, T_{3,0}^{3}\right)$ is isomorphic to $\mathbb{C}^{*} / \pm 1$, with the isomorphism sending $(X, \omega)$ to the residue of $\omega$ at one of the polar nodes. The whole stratum is an orbifold locus of order two in $\Omega \overline{\mathcal{M}}_{2}$, because each $(X, \omega)$ in this stratum has an involution which switches the irreducible components of $X$ and preserves $\omega$.

Choose a symplectic basis $\left\{\alpha_{i}, \beta_{i}\right\}_{i=1}^{2}$ of $H_{1}\left(\Sigma_{2} ; \mathbb{Z}\right)$ such that $\alpha_{i}$ represents the curve $T_{3,0}^{i}$, and let $\alpha_{3} \in H_{1}\left(\Sigma_{2} ; \mathbb{Z}\right)$ be a class representing $T_{3,0}^{3}$. Given a marked stable Abelian differential $(Y, \eta) \in \Omega \mathcal{D}_{2}^{0}\left(T_{3,0}, T_{3,0}^{3}\right)$, the class $\beta_{i}$ determines a class in $H_{1}(Y ; \mathbb{Z})$ which is well defined up to adding multiples of $\alpha_{i}$ and $\alpha_{3}$. This means that

$$
e^{2 \pi i \eta\left(\beta_{i}\right) / \eta\left(\alpha_{i}\right)}
$$


is a well defined complex number because $\eta\left(\alpha_{3}\right)=0$.

Given $(Y, \eta) \in \Omega \mathcal{D}_{2}^{0}\left(T_{3,0}, T_{3,0}^{3}\right)$, define

$$
\begin{array}{ll}
w=\eta\left(\alpha_{1}\right) & x=e^{2 \pi i \eta\left(\beta_{1}\right) / \eta\left(\alpha_{1}\right)} \\
y=e^{2 \pi i \eta\left(\beta_{2}\right) / \eta\left(\alpha_{2}\right)} & z=\left(\int_{I} \eta\right)^{2},
\end{array}
$$

where $I$ is a saddle connection on $(Y, \eta)$ such that $I \cup J(I)$ is homotopic to the curve $T_{3,0}^{3}$. The coordinate $w$ defines an isomorphism $\Omega \mathcal{T}_{2}^{0}\left(T_{3,0}, T_{3,0}^{3}\right) \rightarrow \mathbb{C}$.

Proposition 6.12 The coordinates $(w, x, y, z)$ give a biholomorphic isomorphism of $\Omega \mathcal{D}_{2}^{0}\left(T_{3,0}, T_{3,0}^{3}\right)$ onto its image in $\mathbb{C}^{4}$.

\section{Limits of eigenforms}

\subsection{Introduction}

We now begin the study of the compactification of $X_{D}$. Recall that we have the locus $E_{D} \subset \mathbb{P} \Omega \overline{\mathcal{M}}_{2}$ of eigenforms for real multiplication by $\mathcal{O}_{D}$, and there is the isomorphism $j_{1}: E_{D} \rightarrow X_{D} \backslash P_{D}$ from Proposition 4.4. The inverse of $j_{1}$ extends to an embedding $k_{1}: X_{D} \rightarrow \mathbb{P} \Omega \overline{\mathcal{M}}_{2}$. In this section we will study the closure of $X_{D}$ in $\mathbb{P} \Omega \overline{\mathcal{M}}_{2}$, which we denote by $\bar{X}_{D}$. Our goal is to classify exactly which stable Abelian differentials lie in $\bar{X}_{D} \backslash X_{D}$ and to understand the local structure of $\bar{X}_{D}$ and its strata around these points. More precisely, for each $(X,[\omega]) \in \bar{X}_{D}$ we will:

- Choose a neighborhood of $(X,[\omega])$ in $\mathbb{P} \Omega \overline{\mathcal{M}}_{2}$ of the form $U / \operatorname{Aut}(X,[\omega])$, where $U$ is a neighborhood of $(X,[\omega])$ in an appropriate Dehn space.

- Give local coordinates on $U$ as in Section 6.

- Give explicit equations for the inverse image $\pi^{-1}\left(\bar{X}_{D}\right)$ in $U$.

This procedure will be slightly modified when $(X,[\omega])$ has a nonseparating holomorphic node. In that case, we will only give local coordinates on a hypersurface $V$ in $U$ which we will show contains the inverse image $\pi^{-1}\left(\bar{X}_{D}\right)$ in $U$.

We will see that $\bar{X}_{D}$ in general has non-normal singularities along curves in $\bar{X}_{D} \backslash X_{D}$. To get a less singular compactification, we will pass to the normalization $Y_{D}$ of $\bar{X}_{D}$, which we will study in Section 8 using the results of this section. 
Stratification of $\bar{X}_{\boldsymbol{D}}$ The stratification of $\Omega \overline{\mathcal{M}}_{2}$ which we discussed in Section 6 induces a stratification of $\bar{X}_{D}$. Given a stratum $\Omega \mathcal{M}_{2}^{0}(S, T ; \mathbf{n})$, let $X_{D}(S, T, \mathbf{n})$ denote the stratum,

$$
X_{D}(S, T, \mathbf{n})=\bar{X}_{D} \cap \mathbb{P} \Omega \mathcal{M}_{2}^{0}(S, T, \mathbf{n}),
$$

with the analogous notation if $T$ or $\mathbf{n}$ is omitted. Here is a list of all of these strata with a summary of what we will prove about each one:

(1) $X_{D}(1,1)$ is the stratum of nonsingular eigenforms with two simple zeros. We called this locus $E_{D}(1,1)$ in Section 4.3. It is an open, dense subset of $X_{D}$.

(2) $X_{D}(2)$ is the stratum of nonsingular eigenforms with a double zero, otherwise known as $W_{D}$.

(3) $X_{D}\left(T_{0,1}\right)$ is the stratum of eigenforms consisting of two genus one differentials joined at a node. This is the curve $P_{D}$, which we studied in Section 2.3.

(4-5) $X_{D}\left(T_{1,0}\right)$ is the locus of limiting eigenforms with one nonseparating polar node, containing the stratum $X_{D}\left(T_{1,0} ; 1,1\right)$ as an open dense set and the stratum $X_{D}\left(T_{1,0} ; 2\right)$ as a finite subset. We will show in Section 7.2 that $X_{D}\left(T_{1,0}\right)$ is empty unless $D$ is square. We will show in Section 7.3 that $\bar{X}_{d^{2}}$ is an orbifold around $X_{d^{2}}\left(T_{1,0}\right)$, and $X_{d^{2}}\left(T_{1,0}\right)$ is a suborbifold isomorphic to $\mathbb{H} / \Gamma_{1}(d)$. We will also show that

$$
X_{d^{2}}\left(T_{1,0}\right)=\mathbb{P} \Omega \mathcal{M}_{2}^{0}\left(T_{1,0}\right)(d)
$$

as subsets of $\mathbb{P} \Omega \overline{\mathcal{M}}_{2}$, where $\mathbb{P} \Omega \mathcal{M}_{2}^{0}\left(T_{1,0}, T_{1,0}^{1}\right)(d)$ is the locus of degree $d$ cylinder covering differentials discussed in Section 6.3. We will show that $X_{d^{2}}\left(T_{1,0} ; 2\right)$ consists of the intersection points of $\bar{W}_{d^{2}}$ with $X_{d^{2}}\left(T_{1,0}\right)$ and that these intersections are transverse if $d>3$.

(6) $X_{D}\left(T_{1,0}, T_{1,0}^{1}\right)$ is the stratum of limiting eigenforms with one nonseparating holomorphic node. We will show in Section 7.2 that this stratum is empty unless $D$ is square. We will show in Section 7.4 that $\bar{X}_{d^{2}}$ is an orbifold around $X_{d^{2}}\left(T_{1,0}, T_{1,0}^{1}\right)$, and $X_{d^{2}}\left(T_{1,0}, T_{1,0}^{1}\right)$ is a suborbifold isomorphic to $\mathbb{H} / \Gamma_{1}(d)$. We will also show that

$$
X_{d^{2}}\left(T_{1,0}, T_{1,0}^{1}\right)=\mathbb{P} \Omega \mathcal{M}_{2}^{0}\left(T_{1,0}, T_{1,0}^{1}\right)(d)
$$

as subsets of $\mathbb{P} \Omega \overline{\mathcal{M}}_{2}$, where $\mathbb{P} \Omega \mathcal{M}_{2}^{0}\left(T_{1,0}, T_{1,0}^{1}\right)(d)$ is the $d$-torsion locus introduced in Section 6.4.

(7) $X_{D}\left(T_{2,0}, T_{2,0}^{2}\right)$ is the stratum of limiting eigenforms with one nonseparating holomorphic node and one nonseparating polar node. We will show in Section 7.2 
that this stratum is empty unless $D$ is square. In Section 7.5 we will show that when $D=d^{2}$, this stratum is the finite set,

$$
X_{d^{2}}\left(T_{2,0}, T_{2,0}^{2}\right)=\mathbb{P} \Omega \mathcal{M}_{2}^{0}\left(T_{2,0}, T_{2,0}^{2}\right)(d),
$$

introduced in Section 6.5. We will also show that $\bar{X}_{d^{2}}$ is nonsingular at these points and that each of these points is a transverse intersection point of the closures of the strata $X_{d^{2}}\left(T_{1,0}\right)$ and $X_{d^{2}}\left(T_{1,0}, T_{1,0}^{1}\right)$.

(8-10) The strata $X_{D}\left(T_{2,0} ; 1,1\right), X_{D}\left(T_{2,0} ; 2\right)$, and $X_{D}\left(T_{2,1}\right)$ together consist of the limiting eigenforms with two nonseparating polar nodes, possibly with a separating node. We will show in Section 7.6 that the union of these strata is the union of the curves $C_{\lambda(P)}$, where $P$ is a nondegenerate $Y_{D}$-prototype. We will see that $\bar{X}_{D}$ is in general singular along these curves and can even have several branches passing through them. We will assign a $Y_{D}$-prototypes to each of these branches, and we will give explicit equations for these branches in local coordinates.

(11-12) The strata $X_{D}\left(T_{3,0}\right)$ and $X_{D}\left(T_{3,0}, T_{3,0}^{3}\right)$ together consist of the limiting eigenforms with three nonseparating nodes. We will show in Section 7.7 that $X_{D}\left(T_{3,0}\right)$ is the union of the points $c_{\lambda(P)}$ over all nonterminal $Y_{D}$-prototypes $P$, and $X_{D}\left(T_{3,0}, T_{3,0}^{3}\right)$ is the union of the points $c_{\lambda(P)}$ over all terminal $Y_{D}$-prototypes $P$. We will see that $\bar{X}_{D}$ is in general singular at these points. We will assign $Y_{D}$-prototypes to the branches of $\bar{X}_{D}$ through each $c_{\lambda(P)}$, and we will give equations for these branches in local coordinates.

(13-17) We will show in Section 7.2 that the stratum $X_{D}\left(T_{1,1}\right)$ as well as the four strata consisting of stable Abelian differentials $(X, \omega)$ where $\omega$ vanishes on some irreducible component of $X$ are all empty.

Bundles Over any of the bundles of projective spaces $\mathbb{P} \Omega \overline{\mathcal{M}}_{2}, \mathbb{P} \Omega \mathcal{T}_{2}$, or $\mathbb{P} \Omega \mathcal{D}_{2}(S)$, there is a canonical line bundle $\mathcal{O}(-1)$, whose fiber over a projective class of Abelian differentials, $(X,[\omega])$ is the space of constant multiples of $[\omega]$. Define a Hermitian metric on each of these bundles by defining on the fiber over $(X, \omega)$

$$
h(\eta, \eta)=\int_{X}|\eta|^{2}
$$

This metric is singular over the $(X,[\omega])$ which have infinite area. For use in Section 9, we will give sections for $\mathcal{O}(-1)$ around points in $\bar{X}_{D}$ and calculate the norms of these sections. 


\subsection{Empty strata}

Proposition 7.1 If $D$ is not square, and $(X,[\omega]) \in \bar{X}_{D} \backslash X_{D}$, then $\operatorname{Jac}(X) \cong\left(\mathbb{C}^{*}\right)^{2}$, and the period map $\operatorname{Per}_{\omega}: H_{1}(\operatorname{Jac}(X) ; \mathbb{Z}) \rightarrow \mathbb{C}$ is injective.

Proof If $(X,[\omega]) \in \bar{X}_{D}$, then $(X, \omega) \in \overline{\Omega X}_{D}$ is a nonzero eigenform for real multiplication by $\mathcal{O}_{D}$ by Theorem 5.10 , so $M=H_{1}(\operatorname{Jac}(X) ; \mathbb{Z})$ is a torsion-free $\mathcal{O}_{D}$-module. If $D$ is not square, this implies that the $\mathbb{Z}$-rank of $M$ is even because $M \otimes \mathbb{Q}$ is a $K_{D}$-vector space. This means that either $\operatorname{Jac}(X)$ is compact, or $\operatorname{Jac}(X) \cong\left(\mathbb{C}^{*}\right)^{2}$. Since for any $X \in \bar{X}_{D} \backslash X_{D}$, the $\operatorname{Jacobian} \operatorname{Jac}(X)$ is noncompact, we must have $\operatorname{Jac}(X) \cong\left(\mathbb{C}^{*}\right)^{2}$.

Since $\omega$ is an eigenform, $\operatorname{Per}_{\omega}: M \rightarrow \mathbb{C}$ is $\mathcal{O}_{D^{-}}$-linear, so $K=\operatorname{Ker}\left(\operatorname{Per}_{\omega}\right)$ is an $\mathcal{O}_{D^{-}}$ submodule of $M$. If $K \neq 0$, then $K$ must be a submodule of $M$ of $\mathbb{Z}$-rank two, so $M / K$ would be a finite Abelian group. If $K \neq M$, then this contradicts the fact that $\operatorname{Per}_{\omega}$ embeds $M / K$ into $\mathbb{C}$; if $K=M$, then this contradicts the fact that $\omega$ is nonzero.

Corollary 7.2 If $D$ is not square, then all of the strata in $\bar{X}_{D} \backslash X_{D}$ except for $X_{D}\left(T_{2,0} ; 1,1\right), X_{D}\left(T_{2,0} ; 2\right), X_{D}\left(T_{2,1}\right)$, and $X_{D}\left(T_{3,0}\right)$ are empty.

Proposition 7.3 The stratum $X_{d^{2}}\left(T_{1,1}\right)$ as well as the four strata of $\bar{X}_{d^{2}}$ consisting of stable Abelian differentials $(X, \omega)$ where $\omega$ vanishes on some irreducible component of $X$ are all empty.

Proof We know that each $(X, \omega) \in X_{d^{2}}$ is a degree $d$ branched cover of a genus one Abelian differential by Proposition 4.5. For any stable Abelian differential $(X, \omega) \in$ $\bar{X}_{d^{2}}$, we can take a limit of these branched covers to get a branched covering of degree at most $d$ from $(X, \omega)$ to a genus one stable Abelian differential $(Y, \eta)$. If $(X, \omega) \in X_{d^{2}}\left(T_{1,1}\right)$, then it consists of a one point connected sum of a genus one nonsingular Abelian differential (having finite area) with an infinite cylinder (having infinite area). This is impossible because the same $(Y, \eta)$ cannot be finitely covered by both an Abelian differential with infinite area and one with finite area.

The proofs that the other four strata are empty are all similar, so for concreteness we will show that there is no stable Abelian differential $(X, \omega) \in \bar{X}_{d^{2}}$ which is the one point connected sum,

$$
\left(X_{1}, \omega_{1}\right) \#\left(X_{2}, 0\right),
$$

where $X_{i}$ are elliptic curves with $\omega_{1}$ nonzero. Suppose $(X, \omega)=\lim \left(X_{n}, \omega_{n}\right)$ with $\left(X_{n}, \omega_{n}\right) \in X_{d^{2}}$. We have a degree $d$ branched covering $f_{n}:\left(X_{n}, \omega_{n}\right) \rightarrow\left(F_{n}, v_{n}\right)$ over 
some genus one form $\left(F_{n}, v_{n}\right)$. Taking a subsequence, $\left(F_{n}, v_{n}\right)$ converges to some genus one form $(F, v)$ which is nonsingular because otherwise $E_{n}^{1}$ would converge to a Riemann surface with a node which would contradict the assumption that $X$ has only one node. The injectivity radii of the $\left(F_{n}, v_{n}\right)$ must be uniformly bounded below or else $(F, v)$ would have a node. By Theorem 5.5, for sufficiently large $n$ there is a subsurface $T_{n}$ of $\left(X_{n}, \omega_{n}\right)$ which is homeomorphic to a once-punctured torus and has diameter less that the injectivity radius of $\left(F_{n}, v_{n}\right)$. Thus $f_{n}$ is homotopic to the identity on $T_{n}$.

We now claim that there is no nonconstant branched cover $f: X \rightarrow E$ of a genus two curve over an elliptic curve which sends a closed subsurface $T \subset X$ homeomorphic to a once punctured torus to a contractible subset of $E$, from which the claim will follow. Suppose $f: X \rightarrow E$ is such a branched cover. Let $\gamma \subset E$ be a simple closed curve, bounding a disk $\Delta$, which contains $f(T)$, and let $\gamma^{\prime}$ be the component of $f^{-1}(\gamma)$ which bounds the component $T^{\prime}$ of $f^{-1}(\Delta)$ containing $T$. The subsurface $T^{\prime}$ is also a once punctured torus. By the Riemann-Hurwitz formula applied to the branched cover $X \backslash T^{\prime} \rightarrow E \backslash \Delta$, the restriction of $f$ to $\gamma^{\prime}$ is degree one. Then by the Riemann-Hurwitz formula applied to $T^{\prime} \rightarrow \Delta$, there must be $2 d$ branch points of $f$ contained in $T^{\prime}$, counted with multiplicity, where $d$ is the degree of the restriction of $f$ to $T^{\prime}$. We must have $d>1$ because the restriction of $f$ to $T^{\prime}$ is not a homeomorphism. This is a contradiction because $f$ has only 2 branch points.

\subsection{The strata $X_{D}\left(T_{1,0} ; 1,1\right)$ and $X_{D}\left(T_{1,0}, 2\right)$}

In this section, we will study the strata $X_{D}\left(T_{1,0} ; 1,1\right)$ and $X_{D}\left(T_{1,0}, 2\right)$, whose union is the locus $X_{D}\left(T_{1,0}\right)$, consisting of Abelian differentials with one polar node. Since these are empty when $D$ is not square, we will restrict to the case $D=d^{2}$. The goal of the rest of this section is to prove the following description of these strata.

Theorem 7.4 The locus $X_{d^{2}}\left(T_{1,0}\right)$ is exactly the locus $\mathbb{P} \Omega \mathcal{M}_{2}^{0}\left(T_{1,0}\right)(d)$ of degree $d$ cylinder covering differentials discussed in Section 6.3. The variety $\bar{X}_{d^{2}}$ is an orbifold around $X_{d^{2}}\left(T_{1,0}\right)$ with $X_{d^{2}}\left(T_{1,0}\right)$ a suborbifold isomorphic to $\mathbb{U} / \Gamma_{1}(d)$. The stratum $X_{d^{2}}\left(T_{1,0} ; 2\right) \subset X_{d^{2}}\left(T_{1,0}\right)$ is a finite subset equal to

$$
\bar{W}_{d^{2}} \cap X_{d^{2}}\left(T_{1,0}\right) .
$$

These intersections are transverse if $d>3$.

Local coordinates Let $(X,[\omega]) \in \mathbb{P} \Omega \mathcal{M}_{2}^{0}\left(T_{1,0}\right)$, and choose some marking $f: \Sigma_{2} \rightarrow$ $X$, so that we can regard $(X,[\omega])$ as a point in $\mathbb{P} \Omega \mathcal{T}_{2}^{0}\left(T_{1,0}\right)$. Take a symplectic basis $\left\{\alpha_{i}, \beta_{i}\right\}$ of $H_{1}\left(\Sigma_{2} ; \mathbb{Z}\right)$ as in Section 6.3. On a small neighborhood $U$ of $(X,[\omega])$ in 
$\mathbb{P} \Omega \mathcal{D}_{2}^{0}\left(T_{1,0}\right)$, we can normalize each projective class $(Y,[\eta]) \in U$ so that $\eta\left(\alpha_{1}\right)=1$. This allows us to identify $U$ with the hypersurface $U_{1} \subset \Omega \mathcal{D}_{2}^{0}\left(T_{1,0}\right)$ consisting of all $(Y, \eta)$ with $\eta\left(\alpha_{1}\right)=1$. The restriction of the local coordinates $(v, w, x, y, z)$ from Section 6.3 to $U_{1}$ then gives a system of local coordinates $(w, x, y, z)$ on $U$.

Assume that $U$ is small enough that the natural map $U / \operatorname{Aut}(X,[\omega]) \rightarrow \mathbb{P} \Omega \overline{\mathcal{M}}_{2}$ is an isomorphism onto its image, so that we can regard $U$ as an orbifold coordinate chart around $(X,[\omega])$. By the definition of $\mathbb{P} \mathcal{D}_{2}^{0}\left(T_{1,0}\right)$, for each $(Y, \eta) \in U$, the curve $T_{1,0}^{1}$ is represented by a maximal cylinder $C$ on $(Y, \eta)$. Using Theorem 5.5, by possibly shrinking $U$, we can assume that:

- $\left|\eta\left(\alpha_{2}\right)\right|<\frac{1}{d+1} \operatorname{height}(C)$

- $\left|\eta\left(\beta_{2}\right)\right|<\frac{1}{d+1} \operatorname{height}(C)$

- $\operatorname{Im} \eta\left(\beta_{1}\right)>0$

$X_{d^{2}}\left(T_{1,0}\right)$ in local coordinates We now turn to the question of when our $(X,[\omega])$ lies in the union of strata $X_{d^{2}}\left(T_{1,0}\right)$ and what these strata look like in coordinates around $(X,[\omega])$. We will continue to work in the fixed neighborhood $U$ of some $(X,[\omega]) \in \mathbb{P} \Omega \mathcal{M}_{2}^{0}\left(T_{1,0}\right)$ which we chose above.

For any $(Y, \eta)$ in $U$, we have a marking $f: \Sigma_{2} \rightarrow Y$, which is defined up to Dehn twist around $T_{1,0}$. Choosing a particular marking, we regard the symplectic basis $\left\{\alpha_{i}, \beta_{i}\right\}_{i=1}^{2}$ of $H_{1}\left(\Sigma_{2} ; \mathbb{Z}\right)$ also as a symplectic basis of $H_{1}(Y ; \mathbb{Z})$.

Lemma 7.5 Let $(Y, \eta) \in U \cap X_{d^{2}}$, and let $g:(Y, \eta) \rightarrow(E, v)$ be a primitive, degree $d$, branched cover of a genus one differential $E$. Then there is a basis $\{a, b\}$ of $H_{1}(E ; \mathbb{Z})$ and integers $p, q$, and $r$ such that $\operatorname{gcd}(d, p, q)=1$, and

$$
\begin{array}{ll}
g_{*}\left(\alpha_{1}\right)=d a & g_{*}\left(\beta_{1}\right)=r a+b \\
g_{*}\left(\alpha_{2}\right)=p a & g_{*}\left(\beta_{2}\right)=q a .
\end{array}
$$

Proof There is some primitive $a \in H_{1}(E ; \mathbb{Z})$ such that $g_{*}\left(\alpha_{1}\right)=n a$ for some $n \in \mathbb{N}$. Since $\eta\left(\alpha_{1}\right)=1$, we must have $v(a)>0$. It follows that we can choose some $b \in H_{1}(E ; \mathbb{Z})$ such that $\operatorname{Im} v(b)>0$, and such that $\{a, b\}$ form a basis of $H_{1}(E ; \mathbb{Z})$. The cohomology classes $a$ and $b$ are represented by some closed geodesics $A$ and $B$ on $E$.

We claim that

$$
\operatorname{Im} v(b)>\frac{1}{d+1} \operatorname{height}(C),
$$

where $C$ is the maximal cylinder on $Y$ homotopic to the single curve of $T_{1,0}$. To see this, consider $S=g^{-1}(A) \cap C$. The set $S$ consists of $r$ parallel closed curves $\left\{S_{i}\right\}_{i=1}^{r}$ 
on $C$. Since each point of $B$ has $d$ preimages under $g$, we must have $r \leq d$. Two consecutive curves $S_{i}$ and $S_{i+1}$ bound a cylinder $C_{i}$ which has the same height as the cylinder obtained by cutting $E$ along $A$, so height $\left(C_{i}\right)=\operatorname{Im} v(b)$. There are also two more cylinders, $C_{0}$ and $C_{r}$ bounded by $S_{1}$ and $S_{r}$ respectively and the boundary curves of $C$. These cylinders have height less than $\operatorname{Im} v(b)$ since they are mapped injectively into $E$ by $g$. Together, these cylinders fill out $C$, so

$$
\operatorname{height}(C)=\sum_{i=0}^{r} \operatorname{height}\left(C_{i}\right)<(r+1) \operatorname{Im} v(b) \leq(d+1) \operatorname{Im} v(b),
$$

which proves the claim.

It follows from this claim that $g_{*}\left(\alpha_{2}\right)=p a$ for some $p \in \mathbb{Z}$. To see this, assume that $g_{*}\left(\alpha_{2}\right)=p a+s b$ with $s \neq 0$, then

$$
\begin{array}{rlr}
\frac{1}{d+1} \operatorname{height}(C) & >\left|\eta\left(\alpha_{2}\right)\right| \quad \text { (by the definition of } U \text { ) } \\
& =\left|v\left(g_{*}\left(\alpha_{2}\right)\right)\right| \\
& \geq|s| \operatorname{Im} v(b) \quad \text { (because } v(a) \text { is real) } \\
& >\frac{1}{d+1} \operatorname{height}(C),
\end{array}
$$

a contradiction. Similarly $g_{*}\left(\beta_{2}\right)=q a$ for some $q \in \mathbb{Z}$.

The map $g_{*}: H_{1}(Y ; \mathbb{Z}) \rightarrow H_{1}(E ; \mathbb{Z})$ is onto because $g: Y \rightarrow E$ is primitive. Since we've shown that each basis element of $H_{1}(Y ; \mathbb{Z})$ except $\beta_{1}$ maps to a multiple of $a$, we must have $g_{*}\left(\beta_{1}\right)=r a \pm b$ for some $r \in \mathbb{Z}$, and because $\operatorname{Im} \eta\left(\beta_{1}\right)>0$ and $\operatorname{Im} v(b)>0$, we actually have $g_{*}\left(\beta_{1}\right)=r a+b$. It follows that

$$
g_{*}\left(\alpha_{1}\right) \cdot g_{*}\left(\beta_{1}\right)=(n a) \cdot(r a+b)=n,
$$

and similarly $g_{*}\left(\alpha_{2}\right) \cdot g_{*}\left(\beta_{2}\right)=0$. It follows easily from the fact that $g$ is degree $d$ that

$$
g_{*}\left(\alpha_{1}\right) \cdot g_{*}\left(\beta_{1}\right)+g_{*}\left(\alpha_{2}\right) \cdot g_{*}\left(\beta_{2}\right)=d .
$$

so $n=d$. Because $g$ is primitive, we must have $\operatorname{gcd}(d, p, q)=1$.

Let $\psi: U \rightarrow \mathbb{P} \Omega \mathcal{T}_{2}^{0}\left(T_{1,0}\right)$ be the unplumbing map defined in Section 6.3, which sends $(Y, \eta) \in U$ to the Abelian differential obtained by unplumbing the cylinder on $(Y, \eta)$ homotopic to the curve $T_{1,0}^{1}$. In our local coordinates, $\psi$ is the projection $\psi(w, x, y, z)=(w, x, y, 0)$. In $\mathbb{P} \Omega \mathcal{T}_{2}^{0}\left(T_{1,0}\right)$, let $\mathbb{P} \Omega \mathcal{T}_{2}^{0}\left(T_{1,0}\right)(d)$ be the inverse image of $\mathbb{P} \Omega \mathcal{M}_{2}^{0}\left(T_{1,0}\right)(d)$, the locus of degree $d$ cylinder covering differentials, under the natural projection. 
Theorem 7.6 An Abelian differential $(Y, \eta) \in U \backslash \mathbb{P} \Omega \mathcal{T}_{2}^{0}\left(T_{1,0}\right)$ is in $X_{d^{2}}$ if and only if $\psi(Y, \eta) \in \mathbb{P} \Omega \mathcal{T}_{2}^{0}\left(T_{1,0}\right)(d)$.

Proof Let $(Y, \eta) \in U$, and let $C$ be the maximal closed cylinder on $Y$ containing a closed geodesic $A$ homotopic to the curve of $T_{1,0}$. Let $(Z, v)$ be the stable Abelian differential obtained by cutting $Y$ along $A$ to obtain a surface $\left(Y^{\prime}, \eta^{\prime}\right)$ with geodesic boundaries $A_{1}$ and $A_{2}$, and then gluing in an infinite cylinder to each resulting boundary component of $Y^{\prime}$. The surface $Y^{\prime}$ naturally lies in both $Y$ and $Z$, and $(Z, v)$ is the unplumbing of $(Y, \eta)$ along $C$.

We need to show that $(Y, \eta)$ is a degree $d$ elliptic differential if and only if $(Z, v)$ is a degree $d$ cylinder covering differential.

First, assume that $(Z, v)$ is a degree $d$ cylinder covering differential. Let $g:(Z, v) \rightarrow$ $(D, \xi)$ be a map to a cylinder $(D, \xi)=(\mathbb{C} / \mathbb{Z}, d z)$ realizing $Z$ as a degree $d$ cylinder covering differential. The map $g$ sends the horizontal geodesics $A_{1}$ and $A_{2}$ to horizontal geodesics $B_{1}$ and $B_{2}$ on $C$, and by the Open Mapping Theorem, $B_{1}$ and $B_{2}$ must be distinct (or else the point $p \in Y^{\prime}$ for which $g(p)$ has largest imaginary part would lie in the interior of $Y^{\prime}$, which contradicts the Open Mapping Theorem). The geodesics $B_{1}$ and $B_{2}$ bound a subcylinder $D^{\prime}$ of $D$, and $g$ maps $Y^{\prime}$ onto $D^{\prime}$ with degree $d$. The induced map in homology, $g_{*}: H_{1}\left(Y^{\prime} ; \mathbb{Z}\right) \rightarrow H_{1}\left(D^{\prime} ; \mathbb{Z}\right)$ is onto because $g_{*}: H_{1}(Z ; \mathbb{Z}) \rightarrow H_{1}(D ; \mathbb{Z})$ is onto by primitivity of $g$. Now, we can recover $Y$ by gluing together $A_{1}$ and $A_{2}$ by an appropriate gluing map. By gluing $D^{\prime}$ along $B_{1}$ and $B_{2}$ in a way compatible with the map $g$, we get an elliptic curve $E$ with Abelian differential $\zeta$ and a primitive degree $d$ map $h:(Y, \eta) \rightarrow(E, \zeta)$, realizing $(Y, \eta)$ as a elliptic differential.

Now assume that $(Y, \eta)$ is a degree $d$ elliptic differential. Let $g:(Y, \eta) \rightarrow(E, \zeta)$ be a map realizing $(Y, \eta)$ as an elliptic differential. Take a basis $\{a, b\}$ of $H_{1}(E ; \mathbb{Z})$ as in Lemma 7.5. Let $B=g(A)$, a horizontal closed geodesic on $E$. The geodesics $A$ and $B$ are homologous to $\alpha_{1}$, and $a$ respectively. Since $g_{*}\left(\alpha_{1}\right)=d a$ by Lemma 7.5, the restriction $\left.g\right|_{A}: A \rightarrow B$ is degree $d$, so $g^{-1}(B)=A$.

Cut $E$ along $B$, and call the resulting cylinder with boundary $\left(D^{\prime}, \xi^{\prime}\right)$. Let $(D, \xi)$ be the infinite cylinder obtained by gluing half-infinite cylinders to each boundary component of $\left(D^{\prime}, \xi^{\prime}\right)$. Since $g^{-1}(B)=A$, there is a natural degree $d$ map $h^{\prime}:\left(Y^{\prime}, \eta^{\prime}\right) \rightarrow\left(D^{\prime}, \xi^{\prime}\right)$ and thus a degree $d$ map $h:(Z, v) \rightarrow(D, \xi)$.

To show that $(Z, v)$ is a degree $d$ cylinder covering differential, it just remains to show that $h$ is primitive, and it is enough to show that $h_{*}^{\prime}: H_{1}\left(Y^{\prime} ; \mathbb{Z}\right) \rightarrow H_{1}\left(D^{\prime} ; \mathbb{Z}\right)$ is onto. This follows easily from Lemma 7.5. Since the homology classes $\alpha_{i}$ and $\beta_{2}$ can be represented by closed curves disjoint from $A$, they naturally define classes on $Y^{\prime}$. Then the fact that $\operatorname{gcd}(d, p, q)=1$ from Lemma 7.5, implies that $h_{*}^{\prime}$ is onto. 
Corollary 7.7 We have

$$
X_{d^{2}}\left(T_{1,0}\right)=\mathbb{P} \Omega \mathcal{M}_{2}^{0}\left(T_{1,0}\right)(d) .
$$

Given $(X,[\omega]) \in X_{d^{2}}\left(T_{1,0}\right)$ contained in a sufficiently small neighborhood $U \subset$ $\mathbb{P} \Omega \mathcal{M}_{2}^{0}\left(T_{1,0}\right)$ with coordinates on $U$ as defined above, $\bar{X}_{d^{2}} \cap U$ is cut out by the equations,

$$
\begin{aligned}
& w=\omega\left(\alpha_{2}\right) / \omega\left(\alpha_{1}\right) \\
& x=\omega\left(\beta_{2}\right) / \omega\left(\alpha_{1}\right),
\end{aligned}
$$

and $X_{d^{2}}\left(T_{1,0}\right)$ is cut out by the additional equation $z=0$. If $\omega$ has a double zero, then $\bar{W}_{d^{2}}$ is cut out by the equations (7-2) together with the additional equation $y=0$.

In these coordinates, the foliation $\mathcal{A}_{d^{2}}$ of $X_{d^{2}}$ has leaves given by $z=$ const.

Proof It follows immediately from Theorem 7.6 that $(w, x, y, z) \in U$ is in $X_{d^{2}}$ if and only if $(w, x, y, 0) \in \mathbb{P} \Omega \mathcal{T}_{2}^{0}\left(T_{1,0}\right)(d)$ and $z \neq 0$, and (7-1) follows by taking the closure.

If $(w, x, y, 0) \in \mathbb{P} \Omega \mathcal{T}_{2}^{0}\left(T_{1,0}\right)(d)$, then $\left(w, x, y^{\prime}, 0\right) \in \mathbb{P} \Omega \mathcal{T}_{2}^{0}\left(T_{1,0}\right)(d)$ for any $y^{\prime}$ because being a cylinder covering differential only depends on absolute periods. Furthermore, we must have $w, x \in \frac{1}{d} \mathbb{Z}$ by Lemma 7.5, so the coordinates $w$ and $x$ are locally constant along $\mathbb{P} \Omega \mathcal{T}_{2}^{0}\left(T_{1,0}\right)(d)$. It follows that if $(X,[\omega])=(w, x, y, 0) \in$ $\mathbb{P} \Omega \mathcal{T}_{2}^{0}\left(T_{1,0}\right)(d)$, then around this point, $\mathbb{P} \Omega \mathcal{T}_{2}^{0}\left(T_{1,0}\right)(d)$ is cut out by the equations (7-2) together with $z=0$, and $X_{d^{2}}$ is cut out by the equations (7-2).

The equations for $X_{d^{2}}\left(T_{1,0}\right)$ and $\bar{W}_{d^{2}}$ are clear from the definitions of the coordinates. The leaves of the foliation $\mathcal{A}_{d^{2}}$ are determined by the condition that the absolute periods are locally constant along the leaves, which means in these coordinates that $v, w, x$, and $z$ are all constant. Since we're in $U_{1}$, we have $v=1$, and $w$ and $x$ are locally constant by (7-2). Thus the foliation is given by $z=$ const.

Theorem 7.4 follows directly from this corollary. Note that the statement in Theorem 7.4 that the intersections of $\bar{W}_{d^{2}}$ and $X_{d^{2}}\left(T_{1,0}\right)$ are transverse if $d>3$ follows from the coordinates above together with the fact that $\Gamma_{1}(d)$ is torsion-free if $d>3$.

A section of $\mathcal{O}(-1)$ Recall that we normalized each projective class $(Y,[\eta])$ in $\mathbb{P} \Omega \mathcal{D}_{2}^{0}\left(T_{1,0}\right)$ so that each $\eta\left(\alpha_{1}\right)=1$. We can regard this as defining a section $s$ of the canonical line bundle $\mathcal{O}(-1)$ over $\mathbb{P} \Omega \mathcal{D}_{2}^{0}\left(T_{1,0}\right)$.

Proposition 7.8 The norm of this section $s$ of $\mathcal{O}(-1)$ is given by

$$
h(s, s)=-\frac{1}{2 \pi} \log |z|+w x .
$$


Proof For any genus two Riemann surface $X$ with a symplectic basis $\left\{\alpha_{i}, \beta_{i}\right\}$ of $H_{1}(X ; \mathbb{Z})$, it is well known that for any $\omega \in \Omega(X)$,

$$
\int_{X}|\omega|^{2}=\operatorname{Im}\left(\overline{\omega\left(\alpha_{1}\right)} \omega\left(\beta_{1}\right)+\overline{\omega\left(\alpha_{2}\right)} \omega\left(\beta_{2}\right)\right) .
$$

Equation (7-3) follows from this formula using the fact that $w$ and $x$ are real on $\bar{X}_{d^{2}}$ in these coordinates.

\subsection{The stratum $X_{D}\left(T_{1,0}, T_{1,0}^{1}\right)$}

We now study the stratum $X_{D}\left(T_{1,0}, T_{1,0}^{1}\right)$ of Abelian differentials in $\bar{X}_{D}$ with one nonseparating holomorphic node. Since this stratum is empty if $D$ is not square, we will restrict to the case $D=d^{2}$.

Recall that the $d$-torsion locus $\mathbb{P} \Omega \mathcal{M}_{2}^{0}\left(T_{1,0}, T_{1,0}^{1}\right)(d)$ is the locus of $(X,[\omega]) \in$ $\mathbb{P} \Omega \mathcal{M}_{2}^{0}\left(T_{1,0}, T_{1,0}^{1}\right)$ such that, if we regard $(X,[\omega])$ as a genus one differential with two marked points, then the marked points differ by exactly $d$-torsion on $X$. The goal of this section is to prove the following theorem.

Theorem 7.9 $\bar{X}_{d^{2}}$ is an orbifold around the stratum $X_{d^{2}}\left(T_{1,0}, T_{1,0}^{1}\right)$, which is a suborbifold equal to

$$
\mathbb{P} \Omega \mathcal{M}_{2}^{0}\left(T_{1,0}, T_{1,0}^{1}\right)(d) \cong \mathbb{N} / \Gamma_{1}(d)
$$

Vanishing of periods We now prove that for Abelian differentials $(X,[\omega]) \in X_{d^{2}}$ close to some $\left(X_{0},\left[\omega_{0}\right]\right) \in \bar{X}_{d^{2}}$ which has a holomorphic node, periods along curves of $(X,[\omega])$ which are close to a holomorphic node of $\left(X_{0},\left[\omega_{0}\right]\right)$ must vanish.

Proposition 7.10 Let $S$ be a curve system on $\Sigma_{2}$, let $\pi: \mathbb{P} \Omega \mathcal{D}_{2}(S) \rightarrow \mathbb{P} \Omega \overline{\mathcal{M}}_{2}$ be the natural projection, and let $\left(X_{0},\left[\omega_{0}\right]\right) \in \mathbb{P} \Omega \mathcal{D}_{2}(S) \cap \pi^{-1}\left(\bar{X}_{d^{2}}\right)$ have a nonseparating holomorphic node represented by a homology class $\alpha \in H_{1}\left(\Sigma_{2} ; \mathbb{Z}\right)$. Then for $(X,[\omega]) \in$ $\mathbb{P} \Omega \mathcal{D}_{2}(S)$ sufficiently close to $\left(X_{0},\left[\omega_{0}\right]\right)$, we must have $\omega(\alpha)=0$.

Proof Choose a representative $\omega_{0}$ of the projective class $\left[\omega_{0}\right]$, and suppose there is a sequence $\left(X_{n}, \omega_{n}\right) \rightarrow\left(X_{0}, \omega_{0}\right)$ with $\left(X_{n}, \omega_{n}\right) \in \pi^{-1}\left(X_{d^{2}}\right)$ and $\omega_{n}(\alpha) \neq 0$ for all $n$. There are degree $d$ branched covers $f_{n}:\left(X_{n}, \omega_{n}\right) \rightarrow\left(E_{n}, \eta_{n}\right)$ over elliptic curves. Taking a subsequence, we can assume that $\left(E_{n}, \eta_{n}\right) \rightarrow(E, \eta)$, with $E$ either a cylinder or an elliptic curve and that $f_{n}$ converges to a branched cover $f:\left(X_{0}, \omega_{0}\right) \rightarrow(E, \eta)$ of degree at most $d$. We must then have $\eta \neq 0$. 
The injectivity radius $I\left(E_{n}, \eta_{n}\right)$ of $\left(E_{n}, \eta_{n}\right)$ is bounded by the absolute value of any nonzero period of $\eta_{n}$. Since $\eta_{n}\left(\left(f_{n}\right)_{*} \alpha\right)=\omega_{n}(\alpha)$, and $\omega_{n}(\alpha) \rightarrow 0$, this means that $I\left(E_{n}, \eta_{n}\right) \rightarrow 0$. This is a contradiction because injectivity radius is continuous, and $(E, \eta)$ has nonzero injectivity radius.

Local coordinates Choose some $(X,[\omega]) \in \mathbb{P} \Omega \mathcal{M}_{2}^{0}\left(T_{1,0}, T_{1,0}^{1}\right)$. By choosing a marking $f: \Sigma_{2} \rightarrow X$, we can regard $(X,[\omega])$ as a point in $\mathbb{P} \Omega \mathcal{T}_{2}^{0}\left(T_{1,0}, T_{1,0}^{1}\right)$. Let $\left\{\alpha_{i}, \beta_{i}\right\}_{i=1}^{2}$ be a symplectic basis of $H_{1}\left(\Sigma_{2} ; \mathbb{Z}\right)$ such that $\alpha_{1}$ represents the curve $T_{1,0}^{1}$, as in Section 6.4.

Let $U \subset \mathbb{P} \Omega \mathcal{D}_{2}^{0}\left(T_{1,0}, T_{1,0}^{1}\right)$ be a neighborhood of $(X,[\omega])$ small enough that the natural map $U / \operatorname{Aut}(X,[\omega]) \rightarrow \mathbb{P} \Omega \overline{\mathcal{M}}_{2}$ is an isomorphism onto its image, and $\eta\left(\alpha_{2}\right) \neq 0$ for every $(Y,[\eta]) \in U$. By normalizing each $(Y,[\eta]) \in U$ so that $\eta\left(\alpha_{2}\right)=1$, we can identify $U$ with an open set $U_{1}$ in the hypersurface in $\Omega \mathcal{D}_{2}^{0}\left(T_{1,0}, T_{1,0}^{1}\right)$ of those Abelian differentials $(Z, \zeta)$ such that $\zeta\left(\alpha_{2}\right)=1$. The coordinates $(w, x, y, z)$ from Proposition 6.6 then restrict to local coordinates $(x, y, z)$ on $U$.

$\boldsymbol{X}_{d^{2}}\left(\boldsymbol{T}_{1,0}, \boldsymbol{T}_{1,0}^{\mathbf{1}}\right)$ in local coordinates The set $U$ is not a neighborhood of $(X,[\omega])$ in $\mathbb{P} \Omega \mathcal{D}_{2}^{0}\left(T_{1,0}\right)$, but rather it is a neighborhood of $(X,[\omega])$ in the hypersurface $\mathbb{P} \Omega \mathcal{D}_{2}^{0}\left(T_{1,0}, T_{1,0}^{1}\right)$. Proposition 7.10 implies that in a sufficiently small neighborhood $V$ of $(X,[\omega])$ in $\mathbb{P} \Omega \mathcal{D}_{2}^{0}\left(T_{1,0}\right)$,

$$
V \cap X_{d^{2}} \subset U
$$

This means that we can restrict to this hypersurface and still understand the local structure of $\bar{X}_{d^{2}}$ around $(X,[\omega])$.

Let $\psi: U \rightarrow \mathbb{P} \Omega \mathcal{T}_{2}^{0}\left(T_{1,0}, T_{1,0}^{1}\right)$ be the projection (defined in the proof of Proposition 6.6) which splits each $(X,[\omega]) \in U$ along a union of saddle connections $I \cup J(I)$ which is homotopic to $\alpha_{1}$. In our local coordinates on $U$, this is the map $\phi(x, y, z)=(x, y, 0)$.

Let $\mathbb{P} \Omega \mathcal{T}_{2}^{0}\left(T_{1,0}, T_{1,0}^{1}\right)(d)$ be the inverse image under the natural projection of the $d$-torsion locus $\mathbb{P} \Omega \mathcal{M}_{2}^{0}\left(T_{1,0}, T_{1,0}^{1}\right)(d)$.

Theorem 7.11 $A(Y, \eta) \in U \backslash \mathbb{P} \Omega \mathcal{T}_{2}^{0}\left(T_{1,0}, T_{1,0}^{1}\right)$ is in $X_{d^{2}}$ if and only if $\psi(Y, \eta) \in$ $\mathbb{P} \Omega \mathcal{T}_{2}^{0}\left(T_{1,0}, T_{1,0}^{1}\right)(d)$.

Proof Let $(Y, \eta) \in U$, with saddle connections $I_{1}$ and $I_{2}$, which we split along to form an elliptic curve $(E, v)$. Let $p$ and $q$ be the two zeros of $\eta$. Let $J_{1}$ and $J_{2}$ be the two segments which are the images of the saddle connections $I_{i}$ in $E$. The $J_{i}$ start at points $p_{i}$ and end at points $q_{i}$ such that upon taking the connected sum along 


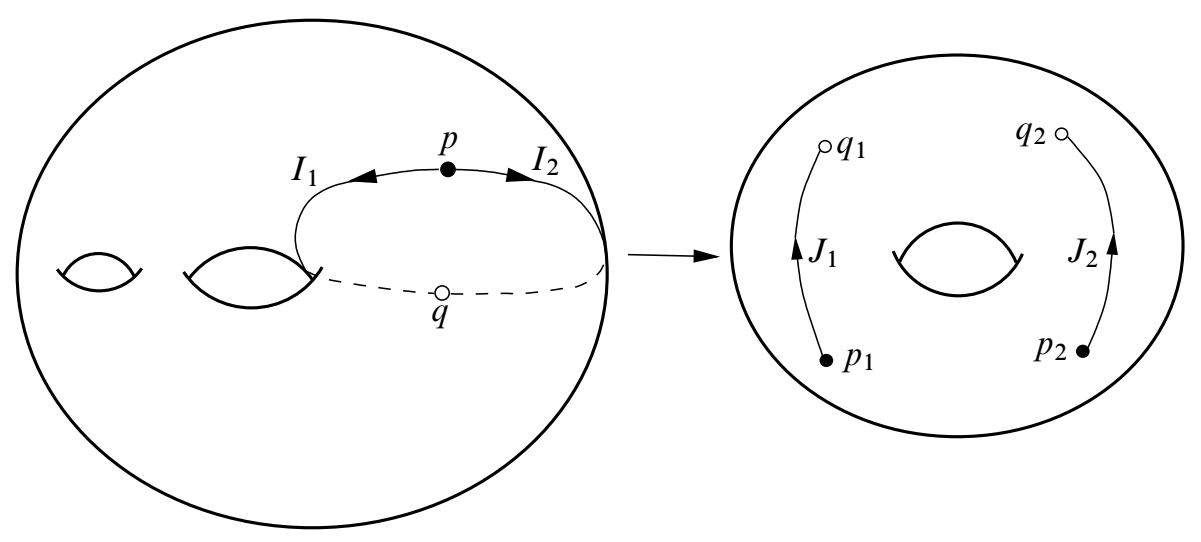

Figure 11: Split along $I_{1} \cup I_{2}$ and then reglue.

$J_{1}$ and $J_{2}$, the $p_{i}$ are identified to form the zero $p$ and the $q_{i}$ are identified to form the zero $q$ (see Figure 11).

We need to show that $(Y, \eta)$ is a degree $d$ elliptic differential if and only if the points $p_{i}$ differ by exactly $d$-torsion in the group law on $E$.

First, suppose that $(Y, \eta)$ is a degree $d$ elliptic differential. Then $(Y, \eta)$ is branched over an elliptic curve by some $g:(Y, \eta) \rightarrow(F, \xi)$. The saddle connections $I_{i}$ must have as their image the same segment $K$ in $F$ because they have the same direction and length, and they start at the same zero $p$. It follows that when we cut $Y$ along $I_{1}$ and $I_{2}$, points which are then glued together to form $E$ map to the same point of $F$ under $g$. Thus $g$ defines an isogeny $g^{\prime}: E \rightarrow F$ of the same degree, sending $p_{1}$ and $p_{2}$ to the same point. This implies that $p_{1}$ and $p_{2}$ differ by $d$-torsion on $E$.

Conversely, if $p$ and $q$ differ by $d$-torsion, there is a degree $d$-isogeny $g:(E, v) \rightarrow$ $(F, \xi)$; it must send points of $J_{1}$ and $J_{2}$ which are glued together to form $Y$ to the same point of $Y$, so we get a branched cover $(Y, \eta) \rightarrow(F, \xi)$ which realizes $\eta$ as a degree $d$ elliptic differential.

Corollary 7.12 The stratum $X_{d^{2}}\left(T_{1,0}, T_{1,0}^{1}\right)$ is exactly $\mathbb{P} \Omega \mathcal{M}_{2}^{0}\left(T_{1,0}, T_{1,0}^{1}\right)(d)$. If we have $(X,[\omega]) \in X_{d^{2}}\left(T_{1,0}, T_{1,0}^{1}\right)$, then in the $(x, y, z)$ coordinates defined above on the neighborhood $U$ of $(X,[\omega])$ in $\mathbb{P} \Omega \mathcal{D}_{2}^{0}\left(T_{1,0}, T_{1,0}^{1}\right)$, the variety $\bar{X}_{d^{2}}$ is cut out by the equation,

$$
d y=a x+b,
$$

for some relatively prime integers $a$ and $b$. 
In these coordinates, the stratum $X_{d^{2}}\left(T_{1,0}, T_{1,0}^{1}\right)$ is cut out by the additional equation $z=0$, and leaves of the foliation $\mathcal{A}_{d^{2}}$ are determined by either of the equivalent equations, $x=$ const or $y=$ const.

Proof It follows immediately from Theorem 7.11 that $(x, y, z) \in U$ is in $X_{d^{2}}$ if and only if $(x, y, 0) \in \mathbb{P} \Omega \mathcal{T}_{2}^{0}\left(T_{1,0}, T_{1,0}^{1}\right)(d)$ and $z \neq 0$. Thus

$$
U \cap \bar{X}_{d^{2}}=\left\{(x, y, z) \in U:(x, y, 0) \in \mathbb{P} \Omega \mathcal{T}_{2}^{0}\left(T_{1,0}, T_{1,0}^{1}\right)(d)\right\},
$$

and $X_{d^{2}}\left(T_{1,0}, T_{1,0}^{1}\right)=\mathbb{P} \Omega \mathcal{M}_{2}^{0}\left(T_{1,0}, T_{1,0}^{1}\right)(d)$ as claimed.

It remains to show that if $(X,[\omega]) \in \mathbb{P} \Omega \mathcal{T}_{2}^{0}\left(T_{1,0}, T_{1,0}^{1}\right)(d)$, then near $(X,[\omega])$, the locus $\mathbb{P} \Omega \mathcal{T}_{2}^{0}\left(T_{1,0}, T_{1,0}^{1}\right)(d)$ is cut out by Equation (7-5). This is because for some $(Y, \eta) \in$ $\mathbb{P} \Omega \mathcal{T}_{2}^{0}\left(T_{1,0}, T_{1,0}^{1}\right)$, a relative period joining the marked points is given by $y=\eta\left(\beta_{1}\right)$, so the marked points differ by $d$-torsion if and only if $d \eta\left(\beta_{1}\right)=a \eta\left(\beta_{2}\right)+b \eta\left(\alpha_{2}\right)$ for some relatively prime integers $a$ and $b$, which is equivalent to (7-5).

The equation for $X_{d^{2}}\left(T_{1,0}, T_{1,0}^{1}\right)$ is clear from the definition of the coordinates, and the equations for the leaves of $\mathcal{A}_{d^{2}}$ hold because either of these equations together with (7-5) implies that the absolute periods are all constant.

Theorem 7.9 follows immediately from this corollary.

\subsection{The stratum $X_{D}\left(T_{2,0}, T_{2,0}^{2}\right)$}

We now study the stratum $X_{D}\left(T_{2,0}, T_{2,0}^{2}\right)$ consisting of limits of eigenforms with one nonseparating polar node and one nonseparating holomorphic node. The arguments in this section are straightforward combinations of those in the previous two sections, so we will omit some of the proofs. This stratum is empty when $D$ is not square, so we will restrict to the case $D=d^{2}$.

Recall that in Section 6.5 we defined the finite set $\mathbb{P} \Omega \mathcal{M}_{2}^{0}\left(T_{2,0}, T_{2,0}^{2}\right)(d)$, consisting of the stable Abelian differentials $f_{q / d} \in \mathbb{P} \Omega \mathcal{M}_{2}^{0}\left(T_{2,0}, T_{2,0}^{2}\right)$, where $q$ is an integer relatively prime to $d$. The following is the main theorem of this section.

Theorem 7.13 The stratum $X_{d^{2}}\left(T_{2,0}, T_{2,0}^{2}\right)$ is the locus $\mathbb{P} \Omega \mathcal{M}_{2}^{0}\left(T_{2,0}, T_{2,0}^{2}\right)(d)$. The variety $\bar{X}_{d^{2}}$ is nonsingular at these points, and each $f_{q / d} \in X_{d^{2}}\left(T_{2,0}, T_{2,0}^{2}\right)$ is a transverse intersection point of the closures of the strata $X_{d^{2}}\left(T_{1,0}\right)$ and $X_{d^{2}}\left(T_{1,0}, T_{1,0}^{1}\right)$. 
Local coordinates Choose some $(X,[\omega]) \in \mathbb{P} \Omega \mathcal{M}_{2}^{0}\left(T_{2,0}, T_{2,0}^{2}\right)$. By choosing a marking $f: \Sigma_{2} \rightarrow X$, we can regard $(X,[\omega])$ as a point in $\mathbb{P} \Omega \mathcal{T}_{2}^{0}\left(T_{2,0}, T_{2,0}^{2}\right)$. Let $\left\{\alpha_{i}, \beta_{i}\right\}_{i=1}^{2}$ be a symplectic basis of $H_{1}\left(\Sigma_{2} ; \mathbb{Z}\right)$ such that $\alpha_{i}$ represents the curve $T_{2,0}^{i}$ in $T_{2,0}$ as in Section 6.5.

Let $U \subset \mathbb{P} \Omega \mathcal{D}_{2}^{0}\left(T_{2,0}, T_{2,0}^{2}\right)$ be a neighborhood of $(X,[\omega])$ small enough that the natural map $U / \operatorname{Aut}(X,[\omega]) \rightarrow \mathbb{P} \Omega \overline{\mathcal{M}}_{2}$ is an isomorphism onto its image. By normalizing each $(Y,[\eta]) \in U$ so that $\eta\left(\alpha_{1}\right)=1$, we can identify $U$ with an open set $U_{1}$ in the hypersurface in $\Omega \mathcal{D}_{2}^{0}\left(T_{2,0}, T_{2,0}^{2}\right)$ consisting of those Abelian differentials $(Z, \zeta)$ such that $\zeta\left(\alpha_{1}\right)=1$. The coordinates $(w, x, y, z)$ from Proposition 6.7 then restrict to coordinates $(x, y, z)$ on $U$.

$X_{d^{2}}\left(T_{2,0}, T_{2,0}^{2}\right)$ in local coordinates Let $\psi: U \rightarrow \mathbb{P} \Omega \mathcal{T}_{2}^{0}\left(T_{2,0}, T_{2,0}^{2}\right)$ be the projection which sends an Abelian differential $(Y, \eta)$ to the one obtained by unplumbing the cylinder on $(Y, \eta)$ homotopic to the curve $T_{2,0}^{1}$ and splitting along the pair of saddle connections $I \cup J(I)$ homotopic to $T_{1,0}^{2}$. In local coordinates, $\psi(x, y, z)=$ $(x, 0,0)$. Let $\mathbb{P} \Omega \mathcal{T}_{2}^{0}\left(T_{2,0}, T_{2,0}^{2}\right)(d)$ be the inverse image under the natural projection to $\mathbb{P} \Omega \mathcal{M}_{2}^{0}\left(T_{2,0}, T_{2,0}^{2}\right)(d)$.

Theorem 7.14 If the neighborhood $U$ of $(X,[\omega])$ is sufficiently small, then a nonsingular $(Y, \eta) \in U$ is in $X_{d^{2}}$ if and only if $\phi(Y, \eta) \in \mathbb{P} \Omega \mathcal{T}_{2}^{0}\left(T_{2,0}, T_{2,0}^{2}\right)(d)$.

The proof of this theorem is a straightforward combination of the proofs of Theorem 7.6 and Theorem 7.11, so we will omit the proof. The idea is that $f_{q / d}$ is a primitive degree $d$ branched cover of a cylinder, and given a $(Y, \eta)$ such that $\psi(Y, \eta)=f_{q / d}$, we can use this cover to exhibit $(Y, \eta)$ as a degree $d$ torus cover.

Corollary 7.15 The stratum $X_{d^{2}}\left(T_{2,0}, T_{2,0}^{2}\right)$ is equal to $\mathbb{P} \Omega \mathcal{M}_{2}^{0}\left(T_{2,0}, T_{2,0}^{2}\right)(d)$. If $(X,[\omega]) \in X_{d^{2}}\left(T_{2,0}, T_{2,0}^{2}\right)$, then in a neighborhood $V$ of $(X,[\omega])$ in $\mathbb{P} \Omega \mathcal{D}_{2}^{0}\left(T_{2,0}\right)$,

$$
V \cap \bar{X}_{d^{2}} \subset \mathbb{P} \Omega \mathcal{D}_{2}^{0}\left(T_{2,0}, T_{2,0}^{2}\right) .
$$

In the coordinates $(x, y, z)$ on a neighborhood $U$ of $(X,[\omega])$ in $\mathbb{P}_{\Omega} \mathcal{D}_{2}^{0}\left(T_{2,0}, T_{2,0}^{2}\right)$, the variety $\bar{X}_{d^{2}}$ is cut out by the equation,

$$
x=\frac{q}{d},
$$

for some integer $q$ relatively prime to $d$.

The intersection of the closure of $X_{d^{2}}\left(T_{1,0}\right)$ with $U$ is cut out by the additional equation $y=0$, and the intersection of the closure of $X_{d^{2}}\left(T_{1,0}, T_{1,0}^{1}\right)$ with $U$ is cut out by the additional equation $z=0$. The leaves of the foliation $\mathcal{A}_{d^{2}}$ of $X_{d^{2}}$ are given by $y=$ const. 
Proof Proposition 7.10 implies directly that in a neighborhood $V$ of $(X,[\omega])$ in $\mathbb{P} \Omega \mathcal{D}_{2}^{0}\left(T_{2,0}\right)$,

$$
V \cap \bar{X}_{d^{2}} \subset \mathbb{P} \Omega \mathcal{D}_{2}^{0}\left(T_{2,0}, T_{2,0}^{2}\right) .
$$

It follows immediately from Theorem 7.14 that $(x, y, z) \in U$ is in $X_{d^{2}}$ if and only if $(x, 0,0) \in \mathbb{P} \Omega \mathcal{T}_{2}^{0}\left(T_{2,0}, T_{2,0}^{2}\right)(d)$ and $y, z \neq 0$. Thus

$$
U \cap \bar{X}_{d^{2}}=\left\{(x, y, z) \in U:(x, 0,0) \in \mathbb{P} \Omega \mathcal{T}_{2}^{0}\left(T_{2,0}, T_{2,0}^{2}\right)(d)\right\}
$$

and $X_{d^{2}}\left(T_{2,0}, T_{2,0}^{2}\right)=\mathbb{P} \Omega \mathcal{M}_{2}^{0}\left(T_{2,0}, T_{2,0}^{2}\right)(d)$ as claimed.

Suppose $(X, \omega) \in \mathbb{P} \Omega \mathcal{T}_{2}^{0}\left(T_{2,0}, T_{2,0}^{2}\right)(d)$. The coordinate $x=\omega\left(\beta_{2}\right)$ measures a relative period on $(X, \omega)$ joining the two marked points which are identified to form a holomorphic node. Since $\omega\left(\alpha_{1}\right)=1$, we have $x=\omega\left(\beta_{2}\right)=q / d$ for some integer $q$ relatively prime to $d$ if and only if the marked points differ by $d$-torsion. Thus $\bar{X}_{d^{2}}$ is cut out by (7-6) as claimed.

The intersection of the closure of $X_{d^{2}}\left(T_{1,0}\right)$ with $U$ is cut out by the additional equation $y=0$ because $y(Y, \eta)=0$ exactly when $(Y, \eta)$ has a polar node, and the intersection of the closure of $X_{d^{2}}\left(T_{1,0}, T_{1,0}^{1}\right)$ with $U$ is cut out by the additional equation $z=0$ because $z(Y, \eta)=0$ exactly when $(Y, \eta)$ has a holomorphic node.

The equation $y=$ const defines the foliation $\mathcal{A}_{d^{2}}$ because this makes the period along $\beta_{1}$ constant, and we have already seen that the other periods are locally constant along $X_{d^{2}}$ with our normalization for $\eta$.

Theorem 7.13 follows directly from this corollary.

A section of $\mathcal{O}(-1)$ Recall that we normalized each projective class $(Y,[\eta])$ in $\mathbb{P} \Omega \mathcal{D}_{2}^{0}\left(T_{2,0}, T_{2,0}^{2}\right)$ so that each $\eta\left(\alpha_{1}\right)=1$. We can regard this as defining a section $s$ of the canonical line bundle $\mathcal{O}(-1)$ over $\mathbb{P} \Omega \mathcal{D}_{2}^{0}\left(T_{2,0}, T_{2,0}^{2}\right)$.

The following follows directly from (7-4) together with the definition of our coordinates.

Proposition 7.16 The norm of this section $s$ of $\mathcal{O}(-1)$ is given by

$$
h(s, s)=-\frac{1}{2 \pi} \log |z| .
$$




\subsection{The strata $X_{D}\left(T_{2,0} ; 1,1\right), X_{D}\left(T_{2,0} ; 2\right)$, and $X_{D}\left(T_{2,1}\right)$}

We now turn to the strata $X_{D}\left(T_{2,0} ; 1,1\right), X_{D}\left(T_{2,0} ; 2\right)$, and $X_{D}\left(T_{2,1}\right)$ which together consist of those limiting eigenforms which have exactly two nonseparating polar nodes, possibly with a separating node.

Recall the definitions from Section 6.6 of the curves $C_{\lambda}$ and the points $p_{\lambda}$ and $w_{\lambda}$ in $\mathbb{P} \Omega \overline{\mathcal{M}}_{2}$. The following is the classification of points in the strata $X_{D}\left(T_{2,0} ; 1,1\right)$, $X_{D}\left(T_{2,0} ; 2\right)$, and $X_{D}\left(T_{2,1}\right)$, which we will prove over the course of this subsection.

Theorem 7.17 The union of the strata $X_{D}\left(T_{2,0} ; 1,1\right), X_{D}\left(T_{2,0} ; 2\right)$, and $X_{D}\left(T_{2,1}\right)$ is equal to the union,

$$
\bigcup_{\lambda} C_{\lambda}
$$

where the union is over all $\lambda$ such that $\lambda=\lambda(P)$ for some nondegenerate $Y_{D}$-prototype $P$. The stratum $X_{D}\left(T_{2,0} ; 2\right)$ is the finite set,

$$
\bigcup_{\lambda} w_{\lambda}
$$

where the union is over all $\lambda$ such that $\lambda=\lambda(P)$ for some $W_{D}$-prototype $P$. The stratum $X_{D}\left(T_{2,1}\right)$ is the finite set,

$$
\bigcup_{\lambda} p_{\lambda}
$$

where the union is over all $\lambda$ such that $\lambda=\lambda(P)$ for some $P_{D}$-prototype $P$. The stratum $X_{D}\left(T_{2,0} ; 1,1\right)$ is the complement of these finite sets in (7-8).

We will also see that in general $\bar{X}_{D}$ is singular along the curves $C_{\lambda}$, and we will give equations for $\bar{X}_{D}$ in local coordinates around $C_{\lambda}$.

Local coordinates Choose an $(X,[\omega])$ in $\mathbb{P} \Omega \mathcal{M}_{2}^{0}\left(T_{2,0} ; 1,1\right), \mathbb{P} \Omega \mathcal{M}_{2}^{0}\left(T_{2,0} ; 2\right)$ or $\mathbb{P} \Omega \mathcal{M}_{2}^{0}\left(T_{2,1}\right)$. By choosing a marking $f: \Sigma_{2} \rightarrow X$, we can regard $(X,[\omega])$ as a point in $\mathbb{P} \Omega \mathcal{T}_{2}^{0}\left(T_{2,0} ; 1,1\right), \mathbb{P} \Omega \mathcal{T}_{2}^{0}\left(T_{2,0} ; 2\right)$, or $\mathbb{P} \Omega \mathcal{T}_{2}^{0}\left(T_{2,1}\right)$.

Our first goal is to show that if $(X,[\omega]) \in \bar{X}_{D}$, then $(X,[\omega])$ lies in the union (7-8). If $(X,[\omega]) \in \bar{X}_{D}$, then $\omega$ is an eigenform for real multiplication of $\mathcal{O}_{D}$ on $\operatorname{Jac}(X)$ by Theorem 5.10, so assume that it is such an eigenform. It follows that the ratio of the residues of the two poles of $\omega$ is real. Thus we can choose a representative $\omega$ of the projective class $[\omega]$ and choose homology classes $\alpha_{1}$ and $\alpha_{2}$ in $H_{1}\left(\Sigma_{2}, \mathbb{Z}\right)$ such that the following properties hold: 
- The $\alpha_{i}$ represent the two nonseparating nodes of $X$.

- $\omega\left(\alpha_{1}\right)=1$.

- $\omega\left(\alpha_{2}\right)=\lambda$ with $\lambda \geq 1$.

If $\lambda>1$, then this choice of the $\alpha_{i}$ and $\omega$ is uniquely determined up to the hyperelliptic involution by these properties. If $\lambda=1$, then there are two possible choices for the $\alpha_{i}$ and $\omega$ up to the hyperelliptic involution, and we choose one arbitrarily (the other one is then obtained by swapping the $\alpha_{i}$ ).

Extend $\left\{\alpha_{1}, \alpha_{2}\right\}$ to a basis $\left\{\alpha_{i}, \beta_{i}\right\}_{i=1}^{2}$ of $H_{1}\left(\Sigma_{2} ; \mathbb{Z}\right)$ as follows:

- If $(X, \omega) \in \mathbb{P} \Omega \mathcal{M}_{2}^{0}\left(T_{2,0} ; 1,1\right)$, let $\beta_{i}$ be an arbitrary pair which is dual to the $\alpha_{i}$ with respect to the intersection pairing.

- If $(X, \omega) \in \mathbb{P} \Omega \mathcal{M}_{2}^{0}\left(T_{2,1}\right)$, let $\beta_{i}$ be a pair dual to the $\alpha_{i}$ such that each $\beta_{i}$ is represented by a simple closed curve disjoint from the separating curve $T_{2,1}^{3}$. This determines each $\beta_{i}$ up to adding a multiple of $\alpha_{i}$.

- If $(X, \omega) \in \mathbb{P} \Omega \mathcal{M}_{2}^{0}\left(T_{2,0} ; 2\right)$, then the horizontal foliation of $(X, \omega)$ consists of two horizontal cylinders separated by a "figure-eight"; let $F \subset \Sigma_{2}$ be the inverse image of this figure-eight with $p$ the singular point of $F$. In $H_{1}\left(\Sigma_{2} ; \mathbb{Z}\right)$, let the $\beta_{i}$ be a pair which is dual to the $\alpha_{i}$ such that $\beta_{1}$ is represented by a simple closed curve which passes through $p$ and is disjoint from $F \backslash p$, and $\beta_{2}$ is represented by a simple closed curve which is disjoint from $F$ (see Figure 12). These conditions determine each $\beta_{i}$ uniquely up to adding a multiple of $\alpha_{i}$.

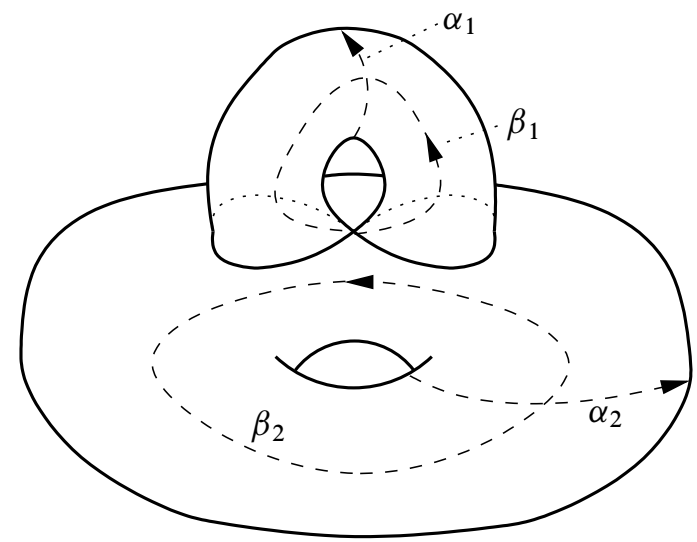

Figure 12: Symplectic basis for $H_{1}\left(\Sigma_{2} ; \mathbb{Z}\right)$

Let $U$ be a small neighborhood of $(X, \omega)$ in $\mathbb{P} \Omega \mathcal{D}_{2}^{0}\left(T_{2,0}\right)$ or $\mathbb{P} \Omega \mathcal{D}_{2}^{0}\left(T_{2,1}\right)$ such that the natural map $U / \operatorname{Aut}(X, \omega) \rightarrow \mathbb{P} \Omega \overline{\mathcal{M}}_{2}$ is an isomorphism onto its image. Also 
choose $U$ small enough so that the conclusion of Theorem 5.9 holds. That is, if $(Y, \eta) \in U$ is an eigenform for real multiplication by $\mathcal{O}_{D}$, then the induced action of $\mathcal{O}_{D}$ on $H_{1}\left(\Sigma_{2} ; \mathbb{Z}\right)$ preserves the subgroup $S$ spanned by the $\alpha_{i}$, giving $S$ the structure of an $\mathcal{O}_{D}$-module.

By normalizing each projective class $(Y,[\eta]) \in U$ so that $\eta\left(\alpha_{1}\right)=1$, we can identify $U$ with an open set $U^{\prime}$ in the hypersurface in $\Omega \mathcal{D}_{2}^{0}\left(T_{2,0}\right)$ or $\Omega \mathcal{D}_{2}^{0}\left(T_{2,1}\right)$ of those Abelian differentials $(Z, \zeta)$ such that $\zeta\left(\alpha_{1}\right)=1$. For any $\mu \in \mathbb{R}$, let

$$
U_{\mu}=\left\{(Y, \eta) \in U: \eta\left(\alpha_{1}\right)=1 \text {, and } \eta\left(\alpha_{2}\right)=\mu\right\},
$$

a hypersurface in $U$. We then have $(X, \omega) \in U_{\lambda}$.

Proposition 7.18 If the neighborhood $U$ of $(X, \omega)$ is taken sufficiently small, then for each $(Y, \eta) \in U \cap X_{D}$, the $\mathcal{O}_{D}$-submodule $S$ of $H_{1}\left(\Sigma_{2} ; \mathbb{Z}\right)$ is a quasi-invertible $\mathcal{O}_{D}$-module with $\left\{\alpha_{1}, \alpha_{2}\right\}$ an admissible basis; furthermore, $U \cap X_{D} \subset U_{\lambda}$.

It follows that $\lambda=\lambda(P)$ for some nondegenerate $Y_{D}$-prototype $P$.

Proof For an Abelian differential $(Y, \eta) \in U$ which is sufficiently close to $(X, \omega)$, the classes $\alpha_{i}$ will be homologous to core curves of very tall horizontal cylinders on $Y$ by Theorem 5.5. Since $\alpha_{i} \cdot \beta_{i}=1$, a curve representing $\beta_{i}$ passes vertically through the cylinder, so the cylinder makes a large positive contribution to $\operatorname{Im} \eta\left(\beta_{i}\right)$. Thus, if $(Y, \eta) \in U$ with $U$ sufficiently small, we must have $\operatorname{Im} \eta\left(\beta_{i}\right)>0$.

The intersection pairing is an unimodular pairing between the $\mathcal{O}_{D}$-modules $S$ and $H_{1}\left(\Sigma_{2} ; \mathbb{Z}\right) / S$, and the $\alpha_{i}$ and $\beta_{i}$ represent bases of these modules dual with respect to this pairing. The period maps associated to $\eta$ and $\operatorname{Im} \eta$ are nonzero $\iota_{1}$-linear maps of these modules to $\mathbb{R}\left(\eta\right.$ is real-valued because $\eta\left(\alpha_{1}\right)=1$ and $\alpha_{2}=\lambda \cdot \alpha_{1}$ for some $\lambda \in K_{D}$ ) which send the $\alpha_{i}$ and the $\beta_{i}$ respectively to positive reals. It then follows from Theorem 3.5 that the $\alpha_{i}$ form an admissible basis of $S$.

By Proposition 3.4, for any $(Y, \eta) \in U \cap X_{D}$ there are integers $a, b$ and $c$ as in the conclusion of that proposition with $a \eta\left(\alpha_{2}\right)^{2}+b \eta\left(\alpha_{2}\right)+c=0$. Since there are only finitely many such integers, and $\eta\left(\alpha_{2}\right) \rightarrow \lambda$ as $(Y, \eta) \rightarrow(X, \omega)$, we must have $\eta\left(\alpha_{2}\right)=\lambda$ for $(Y, \eta)$ sufficiently close to $(X, \omega)$.

Finally, $\lambda=\lambda(P)$ for any $Y_{D}$-prototype $(a, b, c, \bar{q})$ with $a, b$, and $c$ as above.

Corollary 7.19 The strata $X_{D}\left(T_{2,0} ; 1,1\right), X_{D}\left(T_{2,0} ; 2\right)$, and $X_{D}\left(T_{2,1}\right)$ are contained in the union (7-8). 
In the coordinates $(v, w, x, y, z)$ on $\Omega \mathcal{D}_{2}^{0}\left(T_{2,0}\right)$ or $\Omega \mathcal{D}_{2}^{0}\left(T_{2,1}\right)$ from Section 6.6, the subspace $U_{\lambda}$ is cut out by the equations,

$$
v=1 \quad \text { and } \quad w=\lambda
$$

so the $(x, y, z)$ restrict to give local coordinates on $U_{\lambda}$, which send $(Y, \eta) \in U$ to

$$
\begin{aligned}
& x= \begin{cases}\int_{I} \eta & \text { if }(X, \omega) \in \Omega \mathcal{T}_{2}^{0}\left(T_{2,0} ; 1,1\right) ; \\
\left(\int_{I} \eta\right)^{2 / 3} & \text { if }(X, \omega) \in \Omega \mathcal{T}_{2}^{0}\left(T_{2,0} ; 2\right) ; \\
\left(\int_{I} \eta\right)^{2} & \text { if }(X, \omega) \in \Omega \mathcal{T}_{2}^{0}\left(T_{2,1}\right),\end{cases} \\
& y=e^{2 \pi i \eta\left(\beta_{1}\right)}, \\
& z=e^{2 \pi i \eta\left(\beta_{2}\right) / \lambda},
\end{aligned}
$$

where $I$ is a saddle connection joining distinct zeros as in Section 6.6.

Prototypes Now assume that our $(X,[\omega]) \in C_{\lambda}$, where $\lambda=\lambda(P)$ for some nondegenerate $Y_{D}$-prototype $P$ with a small neighborhood $U$ of $(X,[\omega])$ in the Dehn space chosen as above. We will assign a prototype $P(Y, \eta)$ to each Abelian differential $(Y, \eta) \in U \cap X_{D}$. For now, fix such an $(Y, \eta)$.

Recall that we identify two terminal prototypes if they differ by the involution (3-1). We temporarily don't want to make this identification, so we will call a fine prototype a prototype which is defined exactly as in Section 3.1, except without this identification.

The marking $\Sigma_{2} \rightarrow Y$ is well-defined up to Dehn twist around the curves of $T_{2,0}$ or $T_{2,1}$. Choose a particular marking. This allows us to consider the symplectic basis $\left\{\alpha_{i}, \beta_{i}\right\}$ of $H_{1}\left(\Sigma_{2} ; \mathbb{Z}\right)$ as a basis of $H_{1}(Y ; \mathbb{Z})$ as well.

By Proposition 3.4 and Proposition 7.18, there is a unique $\mu$ in $K_{D}$ so that $\mu \cdot \alpha_{1}=\alpha_{2}$, using the real multiplication on $\operatorname{Jac}(Y)$. Since $\eta$ is an eigenform, we know that $\mu^{(1)}=\lambda$ because

$$
\lambda=\eta\left(\alpha_{2}\right)=\eta\left(\mu \cdot \alpha_{1}\right)=\mu^{(1)} \eta\left(\alpha_{1}\right)=\mu^{(1)} .
$$

If $D$ is not square, this means that $\mu$ doesn't depend on $(Y, \eta) \in U$ because $\mu$ is determined by its first embedding. If $D$ is square, however, there can be Abelian differentials close to $(X, \omega)$ with different $\mu^{(2)}$.

Let $a, b$, and $c$ be the integers given by Proposition 3.4. Since $\mathcal{O}_{D}=\mathbb{Z}[a \mu]$, the algebraic integer $a \mu$ acts on $H_{1}(Y ; \mathbb{Z})$ by an integer matrix. 


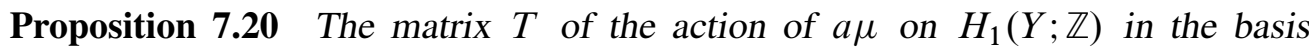
$\left\{\alpha_{1}, \alpha_{2}, \beta_{1}, \beta_{2}\right\}$ is

$$
T=\left(\begin{array}{rrrr}
0 & -c & 0 & q \\
a & -b & -q & 0 \\
0 & 0 & 0 & a \\
0 & 0 & -c & -b
\end{array}\right)
$$

with $a, b$, and $c$ as above, and for some integer $q$.

Proof The matrix $T$ is self-adjoint with respect to the intersection pairing on $H_{1}(Y$; $\mathbb{Z})$, which is equivalent to

$$
\begin{array}{r}
T^{t} J=J T, \\
\text { where } J=\left(\begin{array}{rr}
0 & I \\
-I & 0
\end{array}\right) .
\end{array}
$$

This easily implies that

$$
T=\left(\begin{array}{cc}
A & Q \\
0 & A^{t}
\end{array}\right)
$$

with $Q=-Q^{t}$.

To calculate $A$, we know that

and

$$
a \mu \cdot \alpha_{1}=a \alpha_{2},
$$

so

$$
\begin{aligned}
a \mu \alpha_{2}=a \mu^{2} \alpha_{1} & =(-b \mu-c) \alpha_{1}=-c \alpha_{1}-b \alpha_{2}, \\
A & =\left(\begin{array}{ll}
0 & -c \\
a & -b
\end{array}\right) .
\end{aligned}
$$

Now let $P(Y, \eta)=(a, b, c, \bar{q})$, where $q$ is as in the above Proposition, and $\bar{q}$ is the residue class of $q$, taken modulo $\operatorname{gcd}(a, b, c)$. Similarly, if $(X, \omega)$ has a double zero or a separating node, let $P^{\prime}(Y, \eta)=(a, b, c, \bar{q})$, where $\bar{q}$ is the residue class of $q$, taken modulo $\operatorname{gcd}(a, c)$. Note that the definitions of $P(Y, \eta)$ and $P^{\prime}(Y, \eta)$ involved some choices: there was a choice of which $\beta_{i}$ to take as a basis dual to the $\alpha_{i}$ in $H_{1}\left(\Sigma_{2} ; \mathbb{Z}\right)$, and there was a choice of a marking $\Sigma_{2} \rightarrow Y$ to take up to Dehn twist around $T_{2,0}$ or $T_{2,1}$ which we used to transport the basis of $H_{1}\left(\Sigma_{2} ; \mathbb{Z}\right)$ to $H_{1}(Y ; \mathbb{Z})$.

Theorem 7.21 The quadruple $P(Y, \eta)$ is a fine $Y_{D}$-prototype which does not depend on the choice of the $\beta_{i}$ or the marking $\Sigma_{2} \rightarrow Y$. If $(X, \omega)$ has a double zero or a separating node, then $P^{\prime}(Y, \eta)$ is a fine $W_{D}$ or $P_{D}$-prototype which is also independent of these choices. 
Proof We first show that $P(Y, \eta)$ satisfies the required properties of a prototype. It follows directly from Proposition 3.4 that $b^{2}-4 a c=D$ and $a>0$. We know $N_{\mathbb{Q}}^{K_{D}}(\mu)<0$ because $\left\{\alpha_{1}, \alpha_{2}\right\}$ is an admissible basis of the $\mathcal{O}_{D}$-module $S=\left\langle\alpha_{1}, \alpha_{2}\right\rangle$, so $c<0$. Since $\mathbb{Z}[a \mu]=\mathcal{O}_{D}$ by Proposition 3.4, the matrix in (7-9) must be primitive because the action of $\mathcal{O}_{D}$ on $\operatorname{Jac}(Y)$ is proper; thus $\operatorname{gcd}(a, b, c, \bar{q})=1$. Finally, $a+b+c \leq 1$ because $\mu^{(1)} \geq 1$.

Now we will show that $P(Y, \eta)$ is independent of the choices made. Replacing the $\beta_{i}$ with a new pair which are dual to the $\alpha_{i}$ or changing the marking $\Sigma_{2} \rightarrow Y$ by Dehn twists around $T_{2,0}$ or $T_{2,1}$ produces a new pair $\beta_{i}^{\prime}$ of the form,

$$
\begin{aligned}
& \beta_{1}^{\prime}=\beta_{1}+r \alpha_{1}+s \alpha_{2} \\
& \beta_{2}^{\prime}=\beta_{2}+s \alpha_{1}+t \alpha_{2}
\end{aligned}
$$

for integers $r, s$, and $t$.

The matrix sending the basis $\left\{\alpha_{1}, \alpha_{2}, \beta_{1}, \beta_{2}\right\}$ to $\left\{\alpha_{1}, \alpha_{2}, \beta_{1}^{\prime}, \beta_{2}^{\prime}\right\}$ is

$$
S=\left(\begin{array}{llll}
1 & 0 & r & s \\
0 & 1 & s & t \\
0 & 0 & 1 & 0 \\
0 & 0 & 0 & 1
\end{array}\right) .
$$

If $T$ is the matrix of the endomorphism of $H_{1}(Y ; \mathbb{Z})$ given by multiplication by $a \lambda$ in the old basis. and $T^{\prime}$ is the matrix of the same endomorphism in the new basis, then $T^{\prime}$ is

$$
T^{\prime}=S \cdot T \cdot S^{-1}
$$

which is

$$
\left(\begin{array}{rrrr}
0 & -c & 0 & q^{\prime} \\
a & -b & -q^{\prime} & 0 \\
0 & 0 & 0 & a \\
0 & 0 & -c & -b
\end{array}\right)
$$

where

$$
q^{\prime}=q+a r-b s+c t .
$$

It follows that $q^{\prime} \equiv q(\bmod \operatorname{gcd}(a, b, c))$, so the prototype is independent of the choices.

Now suppose $(X, \omega)$ does have a separating node or a double zero. We must now show that $q$ is well-defined modulo $\operatorname{gcd}(a, c)$. In this case, the choice of $\beta_{i}$ is uniquely determined up to adding a multiple of $\alpha_{i}$ because we imposed additional requirements 
on the $\beta_{i}$. That means that different choices give a new pair $\left\{\beta_{i}^{\prime}\right\}$ of the form (7-10) with $s=0$. Then from $(7-11)$, we see that $q^{\prime} \equiv q(\bmod \operatorname{gcd}(a, c))$.

Finally, if $(X, \omega)$ has a double zero, then $\lambda>1$ by Proposition 6.10, so $a+b+c<0$, and $P^{\prime}(Y, \eta)$ is then a $W_{D}$-prototype.

Remark If $\lambda=1$, then there were also two possible ways to choose the $\alpha_{i}$. In this case, one can check that swapping the $\alpha_{i}$ has the effect of applying the involution (3-1) to the prototypes $P(Y, \eta)$ and $P^{\prime}(Y, \eta)$. Thus they are independent of this choice as ordinary prototypes but not as fine prototypes.

Equations for $\bar{X}_{\boldsymbol{D}}$ in local coordinates Given $(X,[\omega]) \in C_{\lambda}$ and a small neighborhood $U$ of $(X,[\omega])$ in the Dehn space as above, we now give equations for $\bar{X}_{D}$ in $U$ and show that $(X,[\omega])$ is indeed in $\bar{X}_{D}$. Since the prototype $P(Y, \eta)$ is locally constant on $U \cap X_{D}$, we can use these prototypes to label branches of $U \cap X_{D}$. Given a fine $Y_{D}$-prototype $P$, let $V_{P} \subset U$ be the closure in $U$ of

$$
\left\{(Y, \eta) \in U \cap X_{D}: P(Y, \eta)=P\right\},
$$

which in fact lies in $U_{\lambda}$ by Proposition 7.18. If $(X, \omega)$ has a double zero or a separating node, and $P$ is a fine $W_{D}$ or $P_{D}$-prototype (respectively), then let $V_{P}^{\prime}$ the analogous locus where $P^{\prime}(Y, \eta)=P$. In this case, given a fine $W_{D}$-prototype (or $P_{D}$-prototype) $P$,

$$
V_{P}=\bigcup_{Q} V_{Q}^{\prime}
$$

where the union is over all fine $W_{D}$-prototypes (or $P_{D}$-prototypes) which map to $P$.

Let

$$
\left(a^{\prime}, b^{\prime}, c^{\prime}, \bar{q}^{\prime}\right)=(a, b, c, \bar{q}) / \operatorname{gcd}(a, b, c),
$$

and

$$
\left(a^{\prime \prime}, b^{\prime \prime}, c^{\prime \prime}, \bar{q}^{\prime \prime}\right)=(a, b, c, \bar{q}) / \operatorname{gcd}(a, c)
$$

(here we interpret $\bar{q}^{\prime}$ and $\bar{q}^{\prime \prime}$ as elements of $\mathbb{Q} / \mathbb{Z}$ ).

Recall that we have coordinates $(x, y, z)$ on $U_{\lambda}$ defined above.

Theorem 7.22 The locus $V_{P}$ is cut out by the equation,

$$
y^{a^{\prime}}=e^{-2 \pi i q^{\prime}} z^{-c^{\prime}},
$$

and $C_{\lambda}$ is cut out by the equations $y=z=0$.

If $(X, \omega)$ has a double zero or a separating node, then $V_{P}^{\prime}$ is cut out by the equation,

$$
y^{a^{\prime \prime}}=e^{-2 \pi i q^{\prime \prime}} z^{-c^{\prime \prime}}
$$


and $\bar{W}_{D} \cap V_{P}$ or respectively $\bar{P}_{D} \cap V_{P}$ is cut out by the additional equation $x=0$. The leaves of the foliation $\mathcal{A}_{D}$ of $X_{D}$ are given in these coordinates by either of the equivalent equations $y=$ const or $z=$ const.

Proof The proofs of (7-12) and (7-13) are essentially the same, so we will only give the proof of (7-12).

Choose some $(Y, \eta) \in V_{P}$ with a symplectic basis $\left\{\alpha_{i}, \beta_{i}\right\}$ and $\mu \in K_{D}$ as before. Equation (7-9) implies

$$
\begin{aligned}
& a \mu \cdot \beta_{1}=-q \alpha_{2}-c \beta_{2} \\
& a \mu \cdot \beta_{2}=q \alpha_{1}+a \beta_{1}-b \beta_{2} .
\end{aligned}
$$

Since $\eta$ is an eigenform, integrating $\eta$ over both sides gives

$$
\begin{aligned}
& a \lambda \eta\left(\beta_{1}\right)=-q \lambda-c \eta\left(\beta_{2}\right) \\
& a \lambda \eta\left(\beta_{2}\right)=q+a \eta\left(\beta_{1}\right)-b \eta\left(\beta_{2}\right)
\end{aligned}
$$

because $\mu^{(1)}=\lambda$. Dividing (7-16) by $\lambda \operatorname{gcd}(a, b, c)$ and exponentiating yields (7-12).

Now consider $(Y, \eta)$ which lies in $U_{\lambda} \backslash V(y, z)$ and in the locus defined by (7-12) (using the notation $V(\cdot)$ for the zero locus of the enclosed functions). We must show that $(Y, \eta)$ is an eigenform for real multiplication by $\mathcal{O}_{D}$ with prototype $P=(a, b, c, \bar{q})$.

Taking a logarithm of (7-12), we get

$$
a \lambda \eta\left(\beta_{1}\right) \equiv-\lambda \bar{q}-c \eta\left(\beta_{2}\right) \quad(\bmod \lambda \operatorname{gcd}(a, b, c)) .
$$

We can then choose some $q \equiv \bar{q} \bmod \operatorname{gcd}(a, b, c)$ so that (7-16) holds. It then follows automatically from the relation $a \mu^{2}+b \mu+c=0$ that (7-17) holds. We can then define real multiplication of $\mathcal{O}_{D}$ on $\operatorname{Jac}(Y)$ by defining $a \mu$ to act via the matrix $T$ in $(7-9)$. Equations (7-16) and (7-17) then imply $(Y, \eta)$ is actually an eigenform for real multiplication with prototype $P$.

The equations for $\bar{W}_{D}$ and $\bar{P}_{D}$ are obvious from the definition of the coordinates. The leaves of $\mathcal{A}_{D}$ are as claimed because with our normalization the periods $\eta\left(\alpha_{i}\right)$ are constant, and either $y=$ const or $z=$ const implies that both of the $\eta\left(\beta_{i}\right)$ are constant by $(7-12)$.

This also completes the proof of Theorem 7.17 because we explicitly constructed eigenforms near any point of $C_{\lambda(P)}$ for any nondegenerate $Y_{D}$-prototype $P$ in Theorem 7.22 and showed that these are the only possible limits in Corollary 7.19. 
Branches of $\bar{X}_{\boldsymbol{D}}$ through $C_{\lambda}$ Let $p=(X,[\omega]) \in C_{\lambda}$, and let $q \in \mathbb{P} \Omega \mathcal{D}_{2}(S)$ (for the appropriate curve system $S$ ), with $\pi(q)=p$. We have seen that around $q$, the variety $\pi^{-1}\left(\bar{X}_{D}\right)$ is the union of the $V_{P}$ over all fine $Y_{D}$-prototypes $P$ such that $\lambda(P)=\lambda$.

To understand the local structure of $\bar{X}_{D}$ around $p$, we need only to understand how $\operatorname{Aut}(X,[\omega])$ acts on this picture. If $\lambda>1$, then $\operatorname{Aut}(X,[\omega])$ consists only of the hyperelliptic involution which acts trivially on $\mathbb{P} \Omega \mathcal{D}_{2}(S)$. Thus $\pi$ is a local isomorphism around $q$. Note also that there is no distinction between fine and ordinary prototypes when $\lambda>1$.

When $\lambda=1$, we have $\operatorname{Aut}(X,[\omega]) \cong \mathbb{Z} / 2 \oplus \mathbb{Z} / 2$. It is generated by the hyperelliptic involution together with an involution $t$ which interchanges the two nonseparating nodes of $X$ and preserves $\omega$. This involution $t$ acts on $\mathbb{P} \Omega \mathcal{D}_{2}(S)$ by $t(x, y, z)=(x, z, y)$ in the coordinates around $q$ defined above, so $t$ identifies $V_{P}$ with $V_{i(P)}$, where $i$ is the involution of (3-1). Thus $V_{P}$ and $V_{i(P)}$ have the same image in $\mathbb{P} \Omega \overline{\mathcal{M}}_{2}$. We will abuse notation and denote the image of $V_{P}$ in $\mathbb{P} \Omega \overline{\mathcal{M}}_{2}$ by $V_{P}$ as well, where now $P$ is an ordinary $Y_{D}$-prototype. This makes sense because it defines the same object if $P$ is replaced with $i(P)$. This $V_{P}$ is a union of branches of $\bar{X}_{D}$ through $p$. To summarize, whether or not $\lambda=1$, the germ of $\bar{X}_{D}$ around $P$ is the union of the $V_{P}$ where $P$ ranges over all ordinary $Y_{D}$-prototypes such that $\lambda(P)=\lambda$.

If $p=(X,[\omega])$ has a double zero or a separating node, then in the same way - just replacing $V_{P}$ with $V_{P}^{\prime}$ defined above - we define a branch $V_{P}^{\prime}$ of $\bar{X}_{D}$ through $p$, where now $P$ is a $W_{D}$ or $P_{D}$-prototype (respectively). This gives a finer classification of branches then we obtain for generic $p \in C_{\lambda}$

To summarize this discussion, we have the following Corollary of Theorem 7.22.

Corollary 7.23 For any $p \in C_{\lambda}$, the germ of $\bar{X}_{D}$ through $p$ is the union of germs,

$$
\bigcup_{\substack{P \in \mathcal{Y}_{D} \\ \lambda(P)=\lambda}} V_{P} .
$$

Each germ $V_{P}$ consists of mult $(P)$ branches of $\bar{X}_{D}$ through $P$.

If $p=w_{\lambda}$ or $p=p_{\lambda}$, then alternatively the germ of $\bar{X}_{D}$ through $p$ is the union,

$$
\underset{\substack{P \in \mathcal{W}_{D} \\ \lambda(P)=\lambda}}{\bigcup_{P}^{\prime},}
$$

or respectively,

$$
\bigcup_{\substack{P \in \mathcal{P}_{D} \\ \lambda(P)=\lambda}} V_{P}^{\prime} .
$$


In these cases, each of these germs is actually an irreducible branch of $\bar{X}_{D}$ through $p$.

Proof The only parts that don't follow directly from Theorem 7.22 and the discussion above are the statements that $V_{P}$ consists of mult $(P)$ branches and that $V_{P}^{\prime}$ is irreducible. This will follow directly from Proposition 8.1 because

$$
\operatorname{gcd}\left(a^{\prime}, c^{\prime}\right)=\frac{\operatorname{gcd}(a, c)}{\operatorname{gcd}(a, b, c)}=\operatorname{mult}(P)
$$

and $\operatorname{gcd}\left(a^{\prime \prime}, c^{\prime \prime}\right)=1$.

A section of $\mathcal{O}(\mathbf{- 1})$ We normalized each projective class $(Y,[\eta]) \in U_{\lambda}$ so that each $\eta\left(\alpha_{1}\right)=1$. We can regard this as defining a section $s$ of the canonical line bundle $\mathcal{O}(-1)$ over $U_{\lambda}$.

The following follows directly from (7-4) together with the definition of our coordinates.

Proposition 7.24 The norm of this section $s$ of $\mathcal{O}(-1)$ over $U_{\lambda}$ is given by

$$
h(s, s)=-\frac{1}{2 \pi} \log |y|-\frac{\lambda^{2}}{2 \pi} \log |z| .
$$

\subsection{The strata $X_{D}\left(T_{3,0}\right)$ and $X_{D}\left(T_{3,0}, T_{3,0}^{3}\right)$}

We now study Abelian differentials in $\bar{X}_{D}$ with three nonseparating nodes. These consist of the union of the stratum $X_{D}\left(T_{3,0}\right)$, where all of the nodes are polar, and the stratum $X_{D}\left(T_{3,0}, T_{3,0}^{3}\right)$, where one of the nodes is holomorphic. We will prove the following classification of such differentials.

Theorem 7.25 The stratum $X_{D}\left(T_{3,0}\right)$ is the finite union of points,

$$
X_{D}\left(T_{3,0}\right)=\bigcup_{P} c_{\lambda(P)}
$$

where the union is over all nonterminal $Y_{D}$ prototypes $P$.

The stratum $X_{D}\left(T_{3,0}, T_{3,0}^{3}\right)$ is the finite union of points,

$$
X_{D}\left(T_{3,0}, T_{3,0}^{3}\right)=\bigcup_{P} c_{\lambda(P)},
$$

where the union is over all terminal $Y_{D}$-prototypes.

If $P$ is neither degenerate nor terminal, then $c_{\lambda(P)}$ lies in the intersection of the closures of $C_{\lambda(P)}$ and $C_{\lambda\left(P^{+}\right)}$. If $P$ is a degenerate prototype, then $c_{P}$ lies in the intersection 
of the closures of $X_{D}\left(T_{1,0}\right)$ and $C_{\lambda\left(P^{+}\right)}$. If $P$ is a terminal prototype, then $c_{\lambda(P)}$ lies in the intersection of the closures of $C_{\lambda(P)}$ and $X_{D}\left(T_{1,0}, T_{1,0}^{1}\right)$. None of the other curves in $\partial X_{D}$ pass through $c_{\lambda(P)}$.

Local coordinates Fix $(X,[\omega]) \in \mathbb{P} \Omega \mathcal{M}_{2}^{0}\left(T_{3,0}\right)$ or $\mathbb{P} \Omega \mathcal{M}_{2}^{0}\left(T_{3,0}, T_{3,0}^{3}\right)$, and choose a marking $\Sigma_{2} \rightarrow X$ so that we can regard $(X,[\omega])$ as lying in $\mathbb{P} \Omega \mathcal{T}_{2}^{0}\left(T_{3,0}\right)$ or $\mathbb{P} \Omega \mathcal{T}_{2}^{0}\left(T_{3,0}, T_{3,0}^{3}\right)$.

Our first goal is to show that if $(X,[\omega]) \in \bar{X}_{D}$, then $(X,[\omega])$ is one of the points $c_{\lambda(P)}$ as in the statement of Theorem 7.25. If $(X,[\omega]) \in \bar{X}_{D}$, then $\omega$ is an eigenform for real multiplication of $\mathcal{O}_{D}$ on $\operatorname{Jac}(X)$ by Theorem 5.10, so assume that it is such an eigenform. It follows that the ratios of the residues of any two poles of $\omega$ are real, so we can normalize the projective class $[\omega]$ so that all of these residues are real.

Suppose $\omega$ has no holomorphic node. In this case it also has two simple zeros; let $\left\{p_{1}, p_{2}\right\} \in \Sigma_{2}$ be the inverse image of the zeros. Let $\alpha_{i} \in H_{1}\left(\Sigma_{2} \backslash\{p, q\}\right)$ be homology classes representing the three curves $T_{3,0}^{i}$ such that $\omega\left(\alpha_{i}\right) \geq 0$, and choose $\gamma_{i} \in H_{1}\left(\Sigma_{2},\left\{p_{1}, p_{2}\right\}\right)$ so that $\alpha_{i} \cdot \gamma_{j}=\delta_{i j}$ as in Section 6.7. Then the classes,

$$
\begin{aligned}
& \beta_{1}=\gamma_{1}-\gamma_{3} \\
& \beta_{2}=\gamma_{2}+\gamma_{3},
\end{aligned}
$$

in $H_{1}\left(\Sigma_{2} ; \mathbb{Z}\right)$ are dual to $\alpha_{1}$ and $\alpha_{2}$.

If $\omega$ does have a holomorphic node, then let $\alpha_{i} \in H_{1}\left(\Sigma_{2}\right)$ be homology classes representing the three curves $T_{3,0}^{i}$ such that $\omega\left(\alpha_{i}\right) \geq 0$, and let $\beta_{i} \in H_{1}\left(\Sigma_{2} ; \mathbb{Z}\right)$ be dual to $\alpha_{1}$ and $\alpha_{2}$.

Choose a small neighborhood $U$ of $(X,[\omega])$ in $\mathbb{P} \Omega \mathcal{D}_{2}^{0}\left(T_{3,0}\right)$ or $\mathbb{P} \Omega \mathcal{D}_{2}^{0}\left(T_{3,0}, T_{3,0}^{3}\right)$ such that the natural map $U / \operatorname{Aut}(X,[\omega]) \rightarrow \mathbb{P} \Omega \overline{\mathcal{M}}_{2}$ is an isomorphism onto its image and such that for each $(Y,[\eta]) \in U \cap \pi^{-1}\left(X_{D}\right)$, the subgroup $S=\left\langle\alpha_{1}, \alpha_{2}\right\rangle$ of $H_{1}\left(\Sigma_{2} ; \mathbb{Z}\right)$ is preserved by the real multiplication, which is possible by Theorem 5.9.

For the rest of this section, we will adopt the convention that each projective class $(Y,[\eta]) \in U$ is normalized so that $\eta\left(\alpha_{1}\right)=1$, so we can regard $U$ as an open subset of a hypersurface in $\Omega \mathcal{D}_{2}^{0}\left(T_{3,0}\right)$ or $\Omega \mathcal{D}_{2}^{0}\left(T_{3,0}, T_{3,0}^{3}\right)$.

Proposition 7.26 If the neighborhood $U$ of $(X,[\omega])$ is taken sufficiently small, then for each $(Y, \eta) \in U \cap \pi^{-1}\left(X_{D}\right)$, the $\mathcal{O}_{D}$-submodule $S$ of $H_{1}\left(\Sigma_{2}, \mathbb{Z}\right)$ is a quasiinvertible $\mathcal{O}_{D}$-module with $\left\{\alpha_{1}, \alpha_{2}, \alpha_{3}\right\}$ an admissible triple; furthermore, for $(Y, \eta) \in$ $U$, the period maps $P_{\eta}$ and $P_{\omega}: S \rightarrow \mathbb{R}$ are the same. 
Proof Since the curves of $T_{3,0}$ bound a pair of pants, we get the relation

$$
\pm \alpha_{1} \pm \alpha_{2} \pm \alpha_{3}=0
$$

To see that $\left\{\alpha_{1}, \alpha_{2}, \alpha_{3}\right\}$ is an admissible triple, it is enough to show that $\left\{\alpha_{1}, \alpha_{2}\right\}$ is an admissible basis. Just as in the proof of Proposition 7.18, it is enough to show that $\operatorname{Im} \eta\left(\beta_{i}\right)>0$ for $(Y, \eta)$ sufficiently close to $(X, \omega)$. This follows from Theorem 5.5, using the fact that the $\beta_{i}$ pass through tall cylinders, and each cylinder that $\beta_{i}$ passes through contributes positively to $\operatorname{Im} \eta\left(\beta_{i}\right)$ because $\alpha_{j} \cdot \beta_{i} \geq 0$ for each $j$.

Since there are only finitely many admissible triples up to isomorphism by Proposition 3.6, the period $\eta\left(\alpha_{2}\right)$ must be constant on $U$ if $U$ is sufficiently small, and $\eta\left(\alpha_{3}\right)$ must be constant by (7-20). Thus the period maps $P_{\omega}$ and $P_{\eta}$ are the same.

Now since $\left\{\alpha_{1}, \alpha_{2}, \alpha_{3}\right\}$ is an admissible triple, we can reorder them and replace $\omega$ by a constant multiple so that

$$
\omega\left(\alpha_{1}\right)=1, \omega\left(\alpha_{2}\right)=\lambda, \text { and } \omega\left(\alpha_{3}\right)=\lambda-1,
$$

with $\lambda \geq 1$, and $\lambda=\lambda(P)$ for some $Y_{D}$-prototype $P$ by Proposition 3.6. If $\omega$ has no holomorphic node, then there is a unique such choice of the $\alpha_{i}$ and $\omega$ (up to the hyperelliptic involution). Otherwise, there are two such choices because we can swap $\alpha_{1}$ and $\alpha_{2}$. Also when $(X,[\omega])$ has no holomorphic nodes, reorder and possibly change the signs of the $\gamma_{i}$ so that we still have $\alpha_{i} \cdot \gamma_{j}=\delta_{i j}$, and redefine the $\beta_{i}$ as in (7-19) with the new $\gamma_{i}$. When $(X,[\omega])$ has a holomorphic node, reorder the $\beta_{i}$ so that they are still dual to the $\alpha_{i}$.

Thus we see that if $(X,[\omega])$ has three nonseparating nodes and $(X,[\omega]) \in \bar{X}_{D}$, then $(X,[\omega])=c_{\lambda(P)}$ for some $Y_{D}$-prototype $P$ as stated in Theorem 7.25. Also, Proposition 7.26 implies that if $U$ is sufficiently small and if we define for $\mu \in \mathbb{R}$,

$$
U_{\mu}=\left\{(Y, \eta) \in U: \eta\left(\alpha_{1}\right)=1 \text { and } \eta\left(\alpha_{2}\right)=\mu\right\},
$$

then $U \cap \pi^{-1}\left(\bar{X}_{D}\right) \subset U_{\lambda}$, where $\lambda=\omega\left(\alpha_{2}\right)$, by Proposition 7.26.

Recall that in Section 6.7 we defined local coordinates $(v, w, x, y, z)$ on $\Omega \mathcal{D}_{2}^{0}\left(T_{3,0}\right)$. When $\lambda>1$, in these local coordinates, we can identify $U_{\lambda}$ with the subspace defined by $v=1$ and $w=\lambda$. Then on $U_{\lambda}$, the $(x, y, z)$ become

$$
\begin{aligned}
& x=e^{2 \pi i \eta\left(\gamma_{1}\right)} \\
& y=e^{2 \pi i \eta\left(\gamma_{2}\right) / \lambda} \\
& z=e^{2 \pi i \eta\left(\gamma_{3}\right) /(\lambda-1)},
\end{aligned}
$$

and the $(x, y, z)$ are local coordinates on $U_{\lambda}$. 
In Section 6.8 we defined local coordinates $(w, x, y, z)$ on $\Omega \mathcal{D}_{2}^{0}\left(T_{3,0}, T_{3,0}^{3}\right)$. In these local coordinates, we can identify $U_{1}$ with the subspace defined by $w=1$. Then on $U_{1}$, the $(x, y, z)$ become

$$
\begin{aligned}
& x=e^{2 \pi i \eta\left(\beta_{1}\right)} \\
& y=e^{2 \pi i \eta\left(\beta_{2}\right)} \\
& z=\left(\int_{I} \eta\right)^{2},
\end{aligned}
$$

and the $(x, y, z)$ are local coordinates on $U_{1}$.

Prototypes Now assume that our $(X,[\omega])=c_{\lambda}$, where $\lambda=\lambda(P)$ for some $Y_{D^{-}}$ prototype $P$ with a small neighborhood $U$ of $(X,[\omega])$ in the Dehn space chosen as above. We now assign a fine $Y_{D}$-prototype $P(Y,[\eta])$ to each $(Y,[\eta]) \in U \cap \pi^{-1}\left(X_{D}\right)$ as in Section 7.6.

Let $(Y,[\eta]) \in U \cap \pi^{-1}\left(X_{D}\right)$. We have a marking $\Sigma_{2} \rightarrow Y$ which is defined up to Dehn twist around the curve system $T_{3,0}$. Choose a marking $\Sigma_{2} \rightarrow Y$ and use this to transport the symplectic basis $\left\{\alpha_{1}, \alpha_{2}, \beta_{1}, \beta_{2}\right\}$ to $Y$.

There is some $\mu \in K_{D}$ with $\mu^{(1)}=\lambda$ such that $\mu \cdot \alpha_{1}=\alpha_{2}$ and

$$
a \mu^{2}+b \mu+c=0
$$

for integers $a, b$, and $c$ satisfying the properties of Proposition 3.6. The generator $a \mu$ of $\mathcal{O}_{D}$ defines by its action on $\operatorname{Jac}(Y)$ an endomorphism $T$ of $H_{1}(Y ; \mathbb{Z})$ which is given by the matrix (7-9) for some integer $q$. Define the prototype $P(Y, \eta)=(a, b, c, \bar{q})$, where $\bar{q}$ is the reduction of $q$ modulo $\operatorname{gcd}(a, b, c)$. By the same argument as in Theorem 7.21, $P(Y,[\eta])$ is a well-defined fine $Y_{D}$-prototype.

Local coordinates for $\bar{X}_{\boldsymbol{D}}$ Given $(X,[\omega])=c_{\lambda}$ and a small neighborhood $U$ of $(X,[\omega])$ in the Dehn space as above, we now give equations for $\pi^{-1}\left(\bar{X}_{D}\right)$ in $U$ and show that $(X,[\omega])$ is indeed in $\bar{X}_{D}$. For each fine $Y_{D}$-prototype $P$, let $\widetilde{V}_{P} \subset U$ be the closure of

$$
\left\{(Y, \eta) \in U \cap \pi^{-1}\left(X_{D}\right): P(Y, \eta)=P\right\},
$$

which actually lies in $U_{\lambda}$. The following theorem gives explicit equations for $\widetilde{V}_{P}$ in the coordinates $(x, y, z)$ on $U_{\lambda}$.

Theorem 7.27 The locus $\widetilde{V}_{P} \subset U_{\lambda(P)}$ is cut out by the equation

$$
x^{a^{\prime}}=e^{-2 \pi i q^{\prime}} y^{-c^{\prime}} z^{-a^{\prime}-b^{\prime}-c^{\prime}} .
$$


If $P$ is a nondegenerate and nonterminal $Y_{D}$-prototype, then the closure $\bar{C}_{\lambda(P)}$ is cut out by the equations $x=y=0$, and $\bar{C}_{\lambda\left(P^{+}\right)}$is cut out by the equations $x=z=0$.

If $P$ is a degenerate $Y_{D}$-prototype, then the additional equation $y=0$ cuts out $U \cap X_{D}\left(T_{1,0}\right)$, and the equations $x=z=0$ cuts out $\bar{C}_{\lambda\left(P^{+}\right)}$.

If $P$ is a terminal $Y_{D}$-prototype, then the additional equation $z=0$ cuts out $U \cap$ $X_{D}\left(T_{1,0}, T_{1,0}^{1}\right)$, and the equation $x=y=0$ cuts out $\bar{C}_{\lambda(P)}$.

The leaves of the foliation $\mathcal{A}_{D}$ of $X_{D}$ are given in these local coordinates by $y^{\lambda} z^{\lambda-1}=$ const.

Proof If the prototype $P$ is terminal, then (7-21) reduces to (7-12), and the proof that $\widetilde{V}_{\boldsymbol{P}}$ is given by this equation is the same as in Theorem 7.22.

Now assume that $P$ is not terminal. Using the same symplectic basis $\left\{\alpha_{i}, \beta_{i}\right\}$ and $\mu \in K_{D}$ such that $\mu \cdot \alpha_{1}=\alpha_{2}$ as above, Equation (7-9) again implies (7-14) and (7-15). Integrating $\eta$ over both sides yields (7-16) and (7-17), and substituting in (7-19) gives

$$
a \eta\left(\gamma_{1}\right)+\frac{c}{\lambda} \eta\left(\gamma_{2}\right)+\frac{a+b+c}{\lambda-1} \eta\left(\gamma_{3}\right)=-q .
$$

Dividing by $\operatorname{gcd}(a, b, c)$ and exponentiating yields (7-21).

Conversely if $(Y, \eta)$ is in $U_{\lambda}$ minus the coordinate axes and is in the locus defined by (7-21), then (7-22) holds modulo $\operatorname{gcd}(a, b, c)$, and by changing $q$ modulo $\operatorname{gcd}(a, b, c)$, we can ensure that it holds exactly. It then follows that (7-16) and (7-17) hold, and so $(Y, \eta)$ is an eigenform.

The equations for the $C_{\lambda}$ and the other strata are straightforward.

The periods $\eta\left(\alpha_{1}\right)$ and $\eta\left(\alpha_{2}\right)$ are fixed because of our normalization for $\eta$, and the periods $\eta\left(\beta_{1}\right)$ and $\eta\left(\beta_{2}\right)$ are related by the real multiplication, so if one is constant, then the other is. Thus the leaves of the foliation $\mathcal{A}_{D}$ in these coordinates are given by $\eta\left(\beta_{2}\right)=$ const, which is equivalent to $y^{\lambda} z^{\lambda-1}=$ const.

Proof of Theorem 7.25 We saw as a consequence of Proposition 7.26 that each stable Abelian differential in $\bar{X}_{D}$ with three nonseparating nodes is one of the $c_{\lambda(P)}$ for some $Y_{D}$-prototype $P$, and it follows from Theorem 7.27 that these $c_{\lambda(P)}$ are in fact in $\bar{X}_{D}$. The other statements also follow directly from Theorem 7.27. 
Branches of $\bar{X}_{\boldsymbol{D}}$ through $\boldsymbol{c}_{\lambda}$ The varieties cut out by (7-21) have a single branch through the origin, as we will see in Proposition 8.3, so we obtain a bijective correspondence between branches of $\pi^{-1}\left(\bar{X}_{D}\right)$ through $\pi^{-1}\left(c_{\lambda}\right)$ and fine $Y_{D}$-prototypes $P$ such that $\lambda(P)=\lambda$ given by $P \mapsto \widetilde{V}_{P}$. Just as in Section 7.6, the action of $\operatorname{Aut}(X,[\omega])$ identifies branches corresponding to the same ordinary $Y_{D}$-prototype, so we obtain the first statement of the following theorem.

Theorem 7.28 The germ of $\bar{X}_{D}$ through $c_{\lambda}$ is the following union of irreducible branches:

$$
\bigcup_{\substack{P \in \mathcal{Y}_{D} \\ \lambda(P)=\lambda}} \tilde{V}_{P} .
$$

If $P$ is a nondegenerate $Y_{D}$-prototype such that $\lambda(P)=\lambda$, then the branch $\tilde{V}_{P}$ through $c_{\lambda}$ intersects $C_{\lambda}$ in the germ $V_{P}$ of Corollary 7.23. If $P$ is a nonterminal $Y_{D}$-prototype such that $\lambda(P)=\lambda$, then the branch $\widetilde{V}_{P}$ through $c_{\lambda}$ intersects $C_{\lambda\left(P^{+}\right)}$in the germ $V_{P^{+}}$.

Proof Suppose that $P=(a, b, c, \bar{q})$ is nonterminal. Let $(Y, \eta) \in \tilde{V}_{P}$ be a nonsingular Abelian differential close enough to a point $p \in C_{\lambda\left(P^{+}\right)}$that it is contained in some well-defined subgerm $V_{P^{\prime}}$ of $\bar{X}_{D}$ through $p$. We need to show that $P^{\prime}=P^{+}$.

We will continue to use the notation that we used to define the local coordinates around $c_{\lambda}$, so we have classes $\alpha_{1}, \alpha_{2}$, and $\alpha_{3} \in H_{1}(Y ; \mathbb{Z})$ representing the nodes of $c_{\lambda}$ such that $\eta\left(\alpha_{1}\right)=1, \eta\left(\alpha_{2}\right)=\lambda$, and $\eta\left(\alpha_{3}\right)=\lambda-1$; we have $\beta_{1}$, and $\beta_{2} \in H_{1}(Y ; \mathbb{Z})$ such that $\left\{\alpha_{1}, \alpha_{2}, \beta_{1}, \beta_{2}\right\}$ is a symplectic basis of $H_{1}(Y ; \mathbb{Z})$; and we have a $\mu \in K_{D}$ such that $\mu \cdot \alpha_{1}=\alpha_{2}$ and $a \mu^{2}+b \mu+c=0$.

To calculate the prototype $P^{\prime}$, we must choose a symplectic basis $\left\{\alpha_{1}^{\prime}, \alpha_{2}^{\prime}, \beta_{1}^{\prime}, \beta_{2}^{\prime}\right\}$ of $H_{1}(Y ; \mathbb{Z})$ such that the $\alpha_{i}^{\prime}$ are homologous to cylinders on $Y$ representing the nodes of $p \in C_{\lambda\left(P^{+}\right)}$and such that if we set

$$
\tilde{\lambda}=\frac{\eta\left(\alpha_{2}^{\prime}\right)}{\eta\left(\alpha_{1}^{\prime}\right)},
$$

then $\tilde{\lambda} \geq 1$ and $\tilde{\lambda}^{\prime}<0$. Given such a basis, let $\tilde{\mu} \in K_{D}$ be such that $\tilde{\mu} \cdot \alpha_{1}^{\prime}=\alpha_{2}^{\prime}$. Let $a^{\prime}, b^{\prime}$, and $c^{\prime}$ be the unique integers such that

$$
a^{\prime} \tilde{\mu}^{2}+b^{\prime} \tilde{\mu}+c^{\prime}=0
$$

$a^{\prime}>0$, and $\left(b^{\prime}\right)^{2}-4 a^{\prime} c^{\prime}=D$. For $x \in K_{D}$, set $T_{x}$ and $T_{x}^{\prime}$ to be the matrices of the action of $x$ on $H_{1}(Y ; \mathbb{Z})$ in the bases $\left\{\alpha_{i}, \beta_{i}\right\}$ and $\left\{\alpha_{i}^{\prime}, \beta_{i}^{\prime}\right\}$ respectively. Then the 
prototype $P^{\prime}$ is $P^{\prime}=\left(a^{\prime}, b^{\prime}, c^{\prime}, \bar{q}^{\prime}\right)$, where $q^{\prime}$ is the entry in the upper right corner of $T_{a^{\prime} \tilde{\mu}}^{\prime}$

There are two cases to consider, depending on whether or not $\lambda \geq 2$. First suppose that $\lambda \geq 2$, or equivalently $4 a+2 b+c \leq 0$. Define the new symplectic basis of $H_{1}(Y ; \mathbb{Z})$ :

$$
\begin{array}{ll}
\alpha_{1}^{\prime}=\alpha_{1} & \beta_{1}^{\prime}=\beta_{1}+\beta_{2} \\
\alpha_{2}^{\prime}=\alpha_{3}=\alpha_{2}-\alpha_{1} & \beta_{2}^{\prime}=\beta_{2} .
\end{array}
$$

Then $\tilde{\mu}=\mu-1$, and $\tilde{\lambda}=\lambda-1$, which satisfies the required properties. We have

$$
\left(a^{\prime}, b^{\prime}, c^{\prime}\right)=(a, 2 a+b, a+b+c) .
$$

The matrix $T_{a \mu}$ is given by (7-9), and an easy calculation shows that the new matrix is

$$
T_{a^{\prime} \tilde{\mu}}^{\prime}=\left(\begin{array}{cccc}
0-a-b-c & 0 & q \\
a & -2 a-b & -q & 0 \\
0 & 0 & 0 & a \\
0 & 0 & -a-b-c & -2 a-b
\end{array}\right)
$$

so $P^{\prime}=P^{+}$is as claimed.

Now consider the case where $\lambda<2$, or equivalently $4 a+2 b+c>0$. In this case, define a new symplectic basis of $H_{1}(Y ; \mathbb{Z})$ by:

$$
\begin{array}{ll}
\alpha_{1}^{\prime}=\alpha_{2}-\alpha_{1} & \beta_{1}^{\prime}=\beta_{2} \\
\alpha_{2}^{\prime}=\alpha_{1} & \beta_{2}^{\prime}=\beta_{1}+\beta_{2} .
\end{array}
$$

Then

$$
\tilde{\mu}=\frac{1}{\mu-1}=\frac{-a \mu-a-b}{a+b+c},
$$

and $\tilde{\lambda}=1 /(\lambda-1)$ satisfies the required properties that $\tilde{\lambda} \geq 1$ and $\tilde{\lambda}^{\prime}<0$, using the fact that $\lambda>1$ because $P$ is nonterminal. We have

$$
\left(a^{\prime}, b^{\prime}, c^{\prime}\right)=(-a-b-c,-2 a-b,-a),
$$

and an easy calculation shows that the new matrix is

$$
T_{a^{\prime} \tilde{\mu}}^{\prime}=T_{a \mu+a+b}^{\prime}=\left(\begin{array}{cccc}
0 & a & 0 & q \\
-a-b-c & 2 a+b & -q & 0 \\
0 & 0 & 0 & -a-b-c \\
0 & 0 & a & 2 a+b
\end{array}\right) .
$$

Thus $P^{\prime}=P^{+}$as claimed. 
Calculating the prototype of the intersection of $\tilde{V}_{P}$ with $C_{\lambda}$ is much easier. Here we can use the old basis $\left\{\alpha_{i}, \beta_{i}\right\}$ of $H_{1}(Y ; \mathbb{Z})$ to calculate the prototype, and thus it is just $P$.

A section of $\mathcal{O}(-1)$ We normalized each projective class $(Y, \eta) \in U_{\lambda}$ so that each $\eta\left(\alpha_{1}\right)=1$. We can regard this as defining a section $s$ of the canonical line bundle $\mathcal{O}(-1)$ over $U_{\lambda}$.

The following follows directly from (7-4) together with the definition of our coordinates.

Proposition 7.29 The norm of this section $s$ of $\mathcal{O}(-1)$ over $U_{\lambda}$ is given by

$$
h(s, s)=-\frac{1}{2 \pi} \log |x|-\frac{\lambda^{2}}{2 \pi} \log |y|-\frac{(\lambda-1)^{2}}{2 \pi} \log |z| .
$$

\section{Geometric compactification of $X_{D}$}

We have compactified the Hilbert modular surface $X_{D}$ by taking its closure $\bar{X}_{D}$ in $\mathbb{P} \Omega \overline{\mathcal{M}}_{2}$; however, this compactification is not suitable for out purposes. One problem is that $\bar{X}_{D}$ has non-normal singularities, and another is that several cusps of the curves $W_{D}$ and $P_{D}$ can come together to one point in $\bar{X}_{D}$. It turns out that taking the normalization of $Y_{D}$ solves both of these problems and produces a useful compactification which has only quotient singularities.

Definition The geometric compactification $Y_{D}$ of the Hilbert modular surface $X_{D}$ is the normalization of $\bar{X}_{D}$.

This defines $Y_{D}$ as a variety. We will also give $Y_{D}$ the structure of a complex orbifold and of a projective variety in Section 8.1.

In this section, we will study in detail the geometry of $Y_{D}$. In Section 8.1, we will study curves in $Y_{D} \backslash X_{D}$, and their intersections with the curves $\bar{W}_{D}$ and $\bar{P}_{D}$. We show in Section 8.2 that $Y_{D}$ maps to the Baily-Borel compactification $\hat{X}_{D}$ by a map which is the identity on $X_{D}$, and we draw conclusions about the cohomology of $Y_{D}$. In Section 8.3, we study the extension of involution $\tau$ of $X_{D}$ to $Y_{D}$. We will assume that the reader is familiar with the operation of normalization. For a summary of the results about normalization which we use in this section, see Appendix A. 


\subsection{Geometry of $Y_{D}$}

The goal of this subsection is to classify the curves of $Y_{D} \backslash X_{D}$ and to understand the ways in which these curves intersect each other and the curves $\bar{W}_{D}, \bar{P}_{D} \subset Y_{D}$. This is basically a straightforward translation of the results of Section 7 on the local structure of $\bar{X}_{D}$ to the setting of the normalization.

Local normalization of $\bar{X}_{\boldsymbol{D}}$ Normalization is a local operation: constructing the normalization of $\bar{X}_{D}$ just amounts to replacing each small open set with its normalization and then gluing together these normalizations. We saw in Section 7 that the singular points of $\bar{X}_{D}$ are locally modeled on the varieties $V\left(X^{p}-Y^{q}\right)$ and $V\left(X^{p}-Y^{q} Z^{r}\right)$ in $\mathbb{C}^{3}$, so we need to understand the normalizations of these varieties.

Let $\theta_{m}=e^{2 \pi i / m}$. The proof of the following proposition is easy and will be left to the reader.

Proposition 8.1 The normalization of the variety $V=V\left(X^{p}-Y^{q}\right) \subset \mathbb{C}^{3}$ is $\operatorname{gcd}(p, q)$ copies of $\mathbb{C}^{2}$ which map to $V$ by

$$
f_{r}(x, y)=\left(x^{q}, \theta_{\operatorname{gcd}(p, q)}^{r} x^{p}, y\right)
$$

for $0 \leq r<\operatorname{gcd}(p, q)$. It follows that $V$ has $\operatorname{gcd}(p, q)$ branches through each singular point $(0,0, Z)$.

Given $p, q$, and $r \in \mathbb{N}$ relatively prime, let

$$
\begin{aligned}
m & =\frac{p}{\operatorname{gcd}(p, q) \operatorname{gcd}(p, r)} \\
s & \equiv\left(\frac{r}{\operatorname{gcd}(p, r)}\right)^{-1} \bmod \frac{p}{\operatorname{gcd}(p, r)} \\
n & =\frac{-q}{\operatorname{gcd}(p, q)} s,
\end{aligned}
$$

and define $Q(p, q, r)$ to be the normal analytic space $\mathbb{C}^{2} / G$, where $G$ is the order $m$ cyclic group generated by the transformation

$$
(x, y) \mapsto\left(\theta_{m} x, \theta_{m}^{n} y\right) .
$$

Lemma 8.2 If $p q$ and $r$ are relatively prime integers, then the map

$$
f: \mathbb{Z} /\left(\frac{p}{\operatorname{gcd}(p, q)}\right) \times \mathbb{Z} /\left(\frac{p}{\operatorname{gcd}(p, r)}\right) \rightarrow \mathbb{Z} / p
$$

defined by $f(\alpha, \beta)=q \alpha+r \beta$ is surjective, and its kernel is a cyclic group of order $m$ which is generated by $(\operatorname{gcd}(p, r),-q s)$, where $m$ and $s$ are as defined in (8-1). 
Proof It follows from the fact that $p, q$, and $r$ are relatively prime that $f$ is surjective, so the kernel $K$ has order $m$. The element $(\operatorname{gcd}(p, r),-q s)$ clearly belongs to $K$ and has order at least $m$ because $\operatorname{gcd}(p, r)$ has order $m$ in

$$
\mathbb{Z} /\left(\frac{p}{\operatorname{gcd}(p, q)}\right) \text {. }
$$

Thus it is a generator of $K$.

Proposition 8.3 The normalization of the variety $V=V\left(X^{p}-Y^{q} Z^{r}\right) \subset \mathbb{C}^{3}$ is $p: Q(p, q, r) \rightarrow V$, where $p$ is induced by the map $\tilde{p}: \mathbb{C}^{2} \rightarrow V$ defined by

$$
\tilde{p}(x, y)=\left(\begin{array}{c}
x^{q / \operatorname{gcd}(p, q)} y^{r / \operatorname{gcd}(q, r)} \\
x^{p / \operatorname{gcd}(p, q)} \\
y^{p / \operatorname{gcd}(p, r)}
\end{array}\right) .
$$

Proof For $i=1$ or 2 , let $\left(x_{i}, y_{i}\right)$ be a point in $\mathbb{C}^{2}$ minus its coordinate axes. We claim that

$$
\tilde{p}\left(x_{1}, y_{1}\right)=\tilde{p}\left(x_{2}, y_{2}\right),
$$

if and only if the $\left(x_{i}, y_{i}\right)$ are related by the action of $G$. It would then follow that $p$ is the normalization of $V$ because $p$ would be well-defined, finite-to-one, and biholomorphic on the complement of the coordinate axes

It is easy to show that $\tilde{p}\left(x_{1}, y_{1}\right)=\tilde{p}\left(x_{2}, y_{2}\right)$ if the $\left(x_{i}, y_{i}\right)$ are related by $G$. Conversely, suppose the $\left(x_{i}, y_{i}\right)$ have the same image. Then we must have

$$
\left(x_{2}, y_{2}\right)=\left(e^{2 \pi i \alpha \operatorname{gcd}(p, q) / p} x_{1}, e^{2 \pi i \beta \operatorname{gcd}(p, r) / p} x_{2}\right)
$$

for some pair

$$
(\alpha, \beta) \in \mathbb{Z} /\left(\frac{p}{\operatorname{gcd}(p, q)}\right) \times \mathbb{Z} /\left(\frac{p}{\operatorname{gcd}(p, r)}\right)
$$

such that

$$
q \alpha+r \beta \equiv 0 \quad \bmod p .
$$

By Lemma 8.2 there is some $k \in \mathbb{Z}$ such that

$$
\begin{aligned}
& \alpha \equiv k \operatorname{gcd}(p, r) \quad \bmod \frac{p}{\operatorname{gcd}(p, q)} \\
& \beta \equiv-k q s \quad \bmod \frac{p}{\operatorname{gcd}(p, r)},
\end{aligned}
$$

and it follows that the $\left(x_{i}, y_{i}\right)$ are related by an element of $G$.

Since the normalizations of Proposition 8.1 and Proposition 8.3 are either nonsingular or quotient singularities, we can regard $Y_{D}$ as a complex orbifold. 
One nonseparating polar node We now begin to study the curves which make up $Y_{D} \backslash X_{D}$. Define $S_{D}^{1} \subset Y_{D}$ by

$$
S_{D}^{1}=\pi^{-1}\left(X_{D}\left(T_{1,0}\right)\right)
$$

By Proposition 7.1, $S_{D}^{1}$ is empty unless $D$ is square. By Corollary 7.7, points of $S_{d^{2}}^{1}$ correspond precisely to degree $d$ cylinder covering differentials; $S_{d^{2}}^{1}$ is a suborbifold of $Y_{d^{2}}$ by Theorem 7.4, and

$$
S_{d^{2}}^{1} \cong \mathbb{H} / \Gamma_{1}(d)
$$

If $d>3$, then $S_{d^{2}}^{1}$ is actually a submanifold of $Y_{d^{2}}$ because $\Gamma_{1}(d)$ is torsion-free.

There are finitely many intersection points of $\bar{W}_{d^{2}}$ with $S_{d^{2}}^{1}$, and these intersections are transverse if $d>3$ by Theorem 7.4. These intersection points are exactly the one-cylinder cusps of $W_{d^{2}}$.

One nonseparating holomorphic node Define $S_{D}^{2} \subset Y_{D}$ by

$$
S_{D}^{2}=\pi^{-1}\left(X_{D}\left(T_{1,0}, T_{1,0}^{1}\right)\right) \text {. }
$$

By Proposition 7.1, $S_{D}^{2}$ is empty unless $D$ is square. $S_{d^{2}}^{2}$ is a suborbifold of $Y_{d^{2}}$ isomorphic to $\mathbb{T} / \Gamma_{1}(d)$ by Theorem 7.9, and $S_{d^{2}}^{2}$ is a submanifold when $d>3$. The points of $S_{d^{2}}^{2}$ correspond to genus one differentials with two marked points which differ by exactly $d$-torsion identified to form a node.

$\mathrm{SL}_{2} \mathbb{R}$ acts on $S_{d^{2}}^{2}$ with discrete stabilizer, so we can regard $S_{d^{2}}^{2}$ as a degenerate Teichmüller curve. Just as for $W_{d^{2}}$, cusps of $S_{d^{2}}^{2}$ correspond to periodic directions on a given $(X, \omega) \in S_{d^{2}}^{2}$, and we can divide the cusps of $S_{d^{2}}^{2}$ into one and two-cylinder cusps just as for $W_{d^{2}}$.

Given $q \in \mathbb{N}$ such that $0<q \leq d / 2$ and $\operatorname{gcd}(q, d)=1$, let $s_{q / d} \in Y_{d^{2}}$ be the inverse image of $f_{q / d} \in \bar{X}_{d^{2}}$. By Theorem 7.13, these are transverse intersection points of $\bar{S}_{d^{2}}^{1}$ and $\bar{S}_{d^{2}}^{2}$, and these are all of their intersection points, because no other points in our classification of points in $\bar{X}_{d^{2}}$ lie in this intersection. These points $s_{q / d}$ are also exactly the one-cylinder cusps of $S_{d^{2}}^{2}$.

Two or more nodes For each curve $C_{\lambda} \subset \bar{X}_{D}$, we associated a nondegenerate $Y_{D^{-}}$ prototype $P$ to each branch of $\bar{X}_{D}$ through each point $p \in C_{\lambda}$. This means that we can associate a nondegenerate $Y_{D}$-prototype to each point in $\pi^{-1}(p)$ by Theorem A.8.

For any nondegenerate $Y_{D}$-prototype $P$, let $C_{P}^{0} \subset Y_{D}$ be set of points in $\pi^{-1}\left(C_{\lambda(P)}\right)$ associated to $P$. The projection $C_{P}^{0} \rightarrow C_{\lambda(P)}$ is surjective and mult $(P)$-to-one 
by Corollary 7.23. The union of the curves $C_{P}^{0}$ parameterizes those points in $Y_{D}$ representing differentials with two nonseparating nodes and possibly a separating node.

If $\lambda>1$, then $C_{\lambda}$ contains exactly one point $w_{\lambda} \in \bar{W}_{D}$ which represents an Abelian differential with a double zero by Proposition 6.10. We associated a $W_{D}$-prototype to each branch of $\bar{X}_{D}$ through $w_{\lambda}$, and we saw in Corollary 7.23 that $W_{D}$-prototypes $P$ such that $\lambda(P)=\lambda$ correspond bijectively to such branches. Given any $W_{D}$-prototype $P$, let $w_{P} \in Y_{D}$ be the point in $\pi^{-1}\left(w_{\lambda(P)}\right)$ corresponding to the branch associated to $P$. The point $w_{P}$ is a two-cylinder cusp of $W_{D}$, and these are all of the two-cylinder cusps. This correspondence between $W_{D}$-prototypes and two-cylinder cusps of $W_{D}$ is equivalent to the one described in [54]; more precisely, the point $w_{P}$ described here is the cusp $w_{P}$ of Theorem 4.9 .

Similarly, for any $P_{D}$-prototype $P$, define $p_{P} \in Y_{D}$ to be the unique point in $\pi^{-1}\left(p_{\lambda(P)}\right)$ coming from the branch associated to $P$. These points $p_{P}$ are all of the cusps of $P_{D}$.

We know that $W_{D}$ and $P_{D}$ are disjoint since they are $\mathrm{SL}_{2} \mathbb{R}$ orbits. Their intersections with $Y_{D} \backslash X_{D}$ are also disjoint because we have accounted for all of these intersection points above, so it follows that $\bar{P}_{D} \cap \bar{W}_{D}=\varnothing$ in $Y_{D}$.

Suppose $P$ is a $W_{D}$ or a $P_{D}$-prototype that maps to the $Y_{D}$-prototype $Q$. Then the point $w_{P}$ or $p_{P}$ is contained in $C_{Q}^{0}$. This means that if $Q$ is a nonterminal prototype, then $C_{Q}$ contains mult $(P)$ points of $\bar{W}_{D}$ and the same number of points of $\bar{P}_{D}$. If $Q$ is a terminal prototype, then $C_{Q}$ contains a single point of $\bar{P}_{D}$ (because $\operatorname{mult}(Q)=1$ ) and no point of $\bar{W}_{D}$ because no $W_{D}$-prototype maps to a terminal $Y_{D}$-prototype.

Proposition 8.4 The locus $C_{P}^{0}$ is a nonsingular curve in $Y_{D}$. The restriction $\pi: C_{P}^{0} \rightarrow$ $C_{\lambda(P)}$ is an unramified cover of degree mult $(P)$, and $C_{P}^{0}$ is a twice-punctured sphere.

Furthermore, $\bar{W}_{D}$ and $\bar{P}_{D}$ are smooth suborbifolds of $Y_{D}$ which intersect $C_{P}^{0}$ transversely.

Proof Let $p \in C_{P}^{0}$, and $q=\pi(p)$. By Theorem 7.22, the branch $V$ of $\bar{X}_{D}$ through $q$ corresponding to $p$ is isomorphic to the germ at the origin of the variety in $\mathbb{C}^{3}$ cut out by

$$
x^{a^{\prime \prime}}=\theta y^{-c^{\prime \prime}}
$$

for some root of unity $\theta$. In these coordinates, $C_{\lambda(P)}^{0}$ is cut out by $x=y=0$. If $q=w_{\lambda(P)}$ or $q=p_{\lambda(P)}$, then $\bar{W}_{D}$ or $\bar{P}_{D}$ is cut out by $z=0$.

In coordinates $(u, v)$ on a neighborhood of $p$, the map $\pi: Y_{D} \rightarrow X_{D}$ is given by

$$
\pi(u, v)=\left(u^{-c^{\prime \prime}}, \theta^{\prime} u^{a^{\prime \prime}}, v\right),
$$


for some root of unity $\theta^{\prime}$. In these coordinates, $C_{P}$ is cut out by $u=0$, and if $p \in \bar{W}_{D}$ or $\bar{P}_{D}$, then $\bar{W}_{D}$ or $\bar{P}_{D}$ is cut out by $v=0$. The smoothness and transversality statements follow immediately.

Since every point in $C_{\lambda(P)}^{0}$ has mult $(P)$ preimages in $C_{P}$, the projection is unramified of that degree. Thus the locus $C_{P}^{0}$ is a twice-punctured sphere because it is an unramified cover of one.

Finally, $\bar{W}_{D}$ and $\bar{P}_{D}$ are suborbifolds because the coordinates above show that $\bar{W}_{D}$ and $\bar{P}_{D}$ are nonsingular where they cross the $C_{P}^{0}$. These are the only points where $\bar{P}_{D}$ and $\bar{W}_{D}$ meet $Y_{D} \backslash X_{D}$ except for the intersections of $\bar{W}_{D}$ with $S_{D}^{1}$ in the case when $D$ is square, and we have already seen that $\bar{W}_{D}$ is nonsingular there in Theorem 7.4 .

Remark The curves $C_{P}^{0}$ contain no orbifold points of $Y_{D}$. This is clear when $\lambda(P)>1$ because the image $C_{\lambda(P)}$ of $C_{P}^{0}$ in $\mathbb{P} \Omega \overline{\mathcal{M}}_{2}$ contains no orbifold points. When $\lambda(P)=1$, the image $C_{1}$ is an orbifold locus of order two, so a priori $C_{P}$ could be also. This doesn't happen because for any $(X,[\omega]) \subset C_{1}$, the group $\operatorname{Aut}(X,[\omega])$ fixes none of the branches of the inverse image of $\bar{X}_{D}$ through the inverse image of $(X,[\omega])$ in $\mathbb{P} \Omega \mathcal{D}_{2}(S)$.

We now study the closure of $C_{P}^{0}$, which we call $C_{P}$. By Proposition 6.9, $C_{P} \backslash C_{P}^{0}$ consists of points representing differentials $c_{\lambda}$ with three nonseparating nodes, defined in Section 6.6.

By Theorem 7.25 and Theorem 7.28, for every $Y_{D}$-prototype $P$, there is a point $c_{\lambda(P)} \in \bar{X}_{D}$ and a branch of $\bar{X}_{D}$ through $c_{\lambda(P)}$ labeled by $P$. Let $c_{P} \in Y_{D}$ be the point corresponding to the branch labeled with $P$. This gives a bijective correspondence between the points of $Y_{D}$ representing Abelian differentials with three nonseparating nodes and $Y_{D}$-prototypes. Unless $P$ is terminal, all three nodes of $c_{P}$ are polar.

Recall that for a prototype $P=(a, b, c, \bar{q})$, we set

$$
\left(a^{\prime}, b^{\prime}, c^{\prime}\right)=(a, b, c) / \operatorname{gcd}(a, b, c) .
$$

The following proposition describes the structure of $Y_{D}$ in a neighborhood of $c_{P}$.

Proposition 8.5 A neighborhood of the point $c_{P}$, where $P=(a, b, c, \bar{q})$ is a nondegenerate, nonterminal $Y_{D}$-prototype, is isomorphic to a neighborhood $U / G$ of the origin in the quotient singularity $Q\left(a^{\prime},-c^{\prime},-a^{\prime}-b^{\prime}-c^{\prime}\right)$. In coordinates $(u, v)$ on $U$, 
we have

$$
\begin{aligned}
V(u v) & =\left(Y_{D} \backslash X_{D}\right) \cap U \\
V(u) & =C_{P} \cap U \\
V(v) & =C_{P^{+}} \cap U .
\end{aligned}
$$

If $P$ is a degenerate prototype, then $c_{P}$ is a smooth point of $Y_{D}$. In a neighborhood of $c_{P}$, the complement $Y_{D} \backslash X_{D}$ is contained in $\bar{S}_{D}^{1} \cup C_{P^{+}}$, and these curves meet transversely at $c_{P}$.

If $P$ is a terminal prototype, then $c_{P}$ is also a smooth point of $Y_{D}$. In a neighborhood of $c_{P}$, the complement $Y_{D} \backslash X_{D}$ is contained in $\bar{S}_{D}^{2} \cup C_{P}$, and these curves meet transversely at $c_{P}$.

Proof These statements all follow directly from Theorems 7.27 and 7.28, using Proposition 8.3 to translate these theorems into local coordinates on $Y_{D}$.

It follows from this proposition that the order of the orbifold point $c_{P}$ is

$$
m_{P}=\frac{a^{\prime}}{\operatorname{gcd}\left(a^{\prime}, c^{\prime}\right) \operatorname{gcd}\left(a^{\prime}, b^{\prime}+c^{\prime}\right)}=\frac{a}{\operatorname{gcd}(a, c) \operatorname{gcd}(a, b+c) \operatorname{gcd}(a, b, c)} .
$$

Proposition 8.6 The curve $C_{P}$ is a connected rational curve which meets the points $c_{P}$ and $c_{P^{-}}$and no other point $c_{Q}$. If $C_{P}$ is given the structure of an orbifold with orbifold points of order $m_{P}$ at $c_{P}$ and $m_{P^{-}}$at $c_{P^{-}}$, then $C_{P}$ is a suborbifold of $Y_{D}$.

Proof The complement $C_{P} \backslash C_{P}^{0}$ is contained in the union of the points $c_{Q}$, and by Proposition 8.5 it consists of exactly the points $c_{P}$ and $c_{P^{-}}$. It follows from the coordinates in Proposition 8.5 that $C_{P}$ is a suborbifold at these two points.

The curve $C_{P}$ is rational because it is the closure of a twice-punctured sphere.

When $D$ is not square, the operations $P^{+}$and $P^{-}$are defined for all $P$, so the curves $C_{P}$ are divided into finitely many closed chains of rational curves. When $D=d^{2}$, the curves $C_{P}$ are divided into finitely many chains of rational curves joining $\bar{S}_{d^{2}}^{1}$ to $\bar{S}_{d^{2}}^{2}$.

To summarize, we have established the following:

Theorem 8.7 $Y_{D}$ has the following properties:

(1) $Y_{D}$ is a compact, complex orbifold. Its orbifold points are located at the elliptic points of $X_{D}$, the elliptic points of $S_{d^{2}}^{i} \cong \mathbb{H} / \Gamma_{1}(d)$, and the points $c_{P}$ for which $m_{P}>1$. 
(2) The curves $C_{P}, \bar{W}_{D}, \bar{P}_{D}$, and $\bar{S}_{D}^{i}$ are all suborbifolds of $Y_{D}$.

(3) The curves $\bar{S}_{D}^{2}, \bar{W}_{D}$, and $\bar{P}_{D}$ are pairwise disjoint, and $\bar{S}_{D}^{1}$ is disjoint from $\bar{P}_{D}$.

(4) The curves $\bar{S}_{D}^{1}, \bar{S}_{D}^{2}, \bar{W}_{D}$, and $\bar{P}_{D}$ meet the curves $C_{P}$ transversely. These intersections correspond to two cylinder cusps of $S_{D}^{2}$ and $W_{D}$. If $D=d^{2}$ with $d>3$, then $\bar{W}_{d^{2}}$ intersects $S_{d^{2}}^{1}$ transversely.

(5) The point $w_{P} \in \bar{W}_{D}$ lies on the curve $C_{Q}$, where $Q$ is the $Y_{D}$-prototype associated to $P$, and $w_{P}$ lies on no other curve $C_{q^{\prime}}$. This gives a bijective correspondence between the intersection points of $\bar{W}_{D}$ with $C_{Q}$ and the set of $W_{D}$-prototypes associated to the $Y_{D}$-prototype $Q$. Similarly, the intersection points of $\bar{P}_{D}$ with $C_{P}$ correspond bijectively in the same way to the set of $P_{D}$-prototypes associated to the $Y_{D}$-prototype $P$.

If $P$ is neither initial nor terminal, then $C_{P}$ meets $W_{D}$ and $P_{D}$ in mult $(P)$ points.

If $P$ is terminal, then $C_{P}$ meets $\bar{S}_{d^{2}}^{2}$ and $\bar{P}_{D}$ once each and is disjoint from $\bar{W}_{D}$.

If $P$ is initial, then $C_{P}$ meets $\bar{S}_{d^{2}}^{1}, \bar{P}_{D}$, and $\bar{W}_{D}$ once each.

(6) The curves $\bar{S}_{d^{2}}^{1}$ and $\bar{S}_{d^{2}}^{2}$ meet transversely in the $N$ points $s_{q / d}$, where

$$
N= \begin{cases}1, & \text { if } d=2 ; \\ \frac{1}{2} \phi(d), & \text { if } d>2\end{cases}
$$

(here $\phi$ is the Euler phi-function).

Corollary 8.8 If $D$ is not square, then $W_{D}$ and $P_{D}$ have the same number of cusps. The number of cusps of $P_{d^{2}}$ is equal to the number of two-cylinder cusps of $W_{d^{2}}$ plus the number of two-cylinder cusps of $S_{d^{2}}^{2}$.

Example: $Y_{17}$ We will now illustrate these results in some specific cases. Most of the prototypes which arise will have $\bar{q}=0$, and we will abbreviate those prototypes by omitting $q$.

There are five $Y_{17}$ prototypes:

$$
(1,1,-4), \quad(2,-3,-1), \quad(2,-1,-2), \quad(1,-3,-2), \quad(1,-1,-4) .
$$

They form one orbit under the operation $P \mapsto P^{+}$, with each prototype being sent to the next on the list. We have mult $(2,-1,-2)=2$, so $C_{(2,-1,-2)}$ meets $\bar{W}_{17}$ in the points $w_{(2,-1,-2,0)}$ and $w_{(2,-1,2,1)}$ and $\bar{P}_{17}$ in the points $p_{(2,-1,-2,0)}$ and $p_{(2,-1,2,1)}$. The other prototypes have multiplicity one, so the other curves $C_{P}$ meet $\bar{W}_{17}$ and $\bar{P}_{17}$ 
once each. Since $m_{P}=1$ for each $P$, none of the points $c_{P}$ are orbifold points, and the $C_{P}$ meet each other transversely.

This configuration is shown in Figure 13. The curves $C_{P}$ form the pentagon and are marked by their prototypes. The curves representing $\bar{W}_{17}$ and $\bar{P}_{17}$ in this figure accurately represent the connected components of these curves and their intersections with the $C_{P}$.

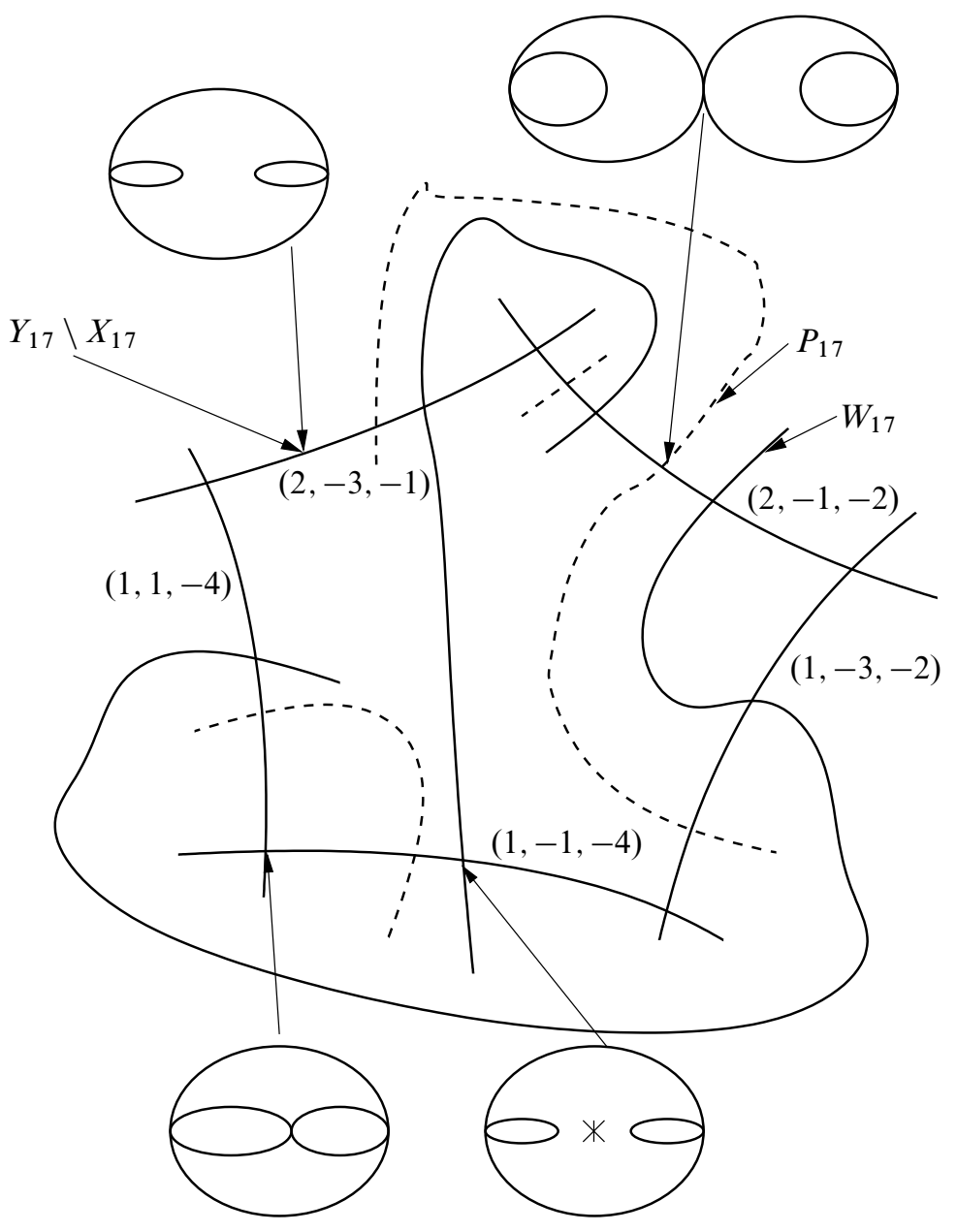

Figure 13: $Y_{17}$

Example: $Y_{12}$ There are three $Y_{12}$ prototypes:

$$
(2,-2,-1), \quad(1,-2,-2), \quad(1,0,-3) .
$$


They form one orbit under the operation $P \mapsto P^{+}$. We have $m_{(2,-2,-1)}=2$, so the point $c_{(2,-2,-1)}$ is an orbifold point of order two in the intersection of $C_{(1,-2,-2)}$ and $C_{(2,-2,-1)}$. The other two points $c_{P}$ are nonsingular. Each of these prototypes has multiplicity one, so $W_{12}$ and $P_{12}$ each have three cusps and intersect each $C_{P}$ once.

Example: $Y_{25}$ There are nine $Y_{25}$-prototypes, which are divided into two orbits under $P \mapsto P^{+}$:

$$
(1,-5,0), \quad(1,-3,-4), \quad(1,-1,-6), \quad(1,1,-6), \quad(1,3,-4),
$$

and $\quad(2,-5,0), \quad(2,-1,-3), \quad(2,-3,-2), \quad(2,1,-3)$.

The prototypes after the first one on each list are nondegenerate and correspond to seven curves $C_{P}$ in $Y_{25}$. Each of these prototypes has $m_{P}=1$, so none of the $c_{P}$ are orbifold points. The curve $W_{25}$ has six two-cylinder cusps; it meets $C_{(2,-3,-2)}$ twice and the other four curves $C_{P}$ corresponding to nonterminal prototypes once each. $W_{25}$ also has two one-cylinder cusps (see Section 13, McMullen [54], or Lelièvre and Royer [47] for formulas for the number of one-cylinder cusps) corresponding to two intersections with $S_{25}^{1}$. The curve $\bar{P}_{25}$ also meets $C_{(2,-3,-2)}$ twice and each of the other $C_{P}$ once each.

Each of the two-cylinder cusps of $W_{25}$ can be represented by a square-tiled surface with five squares having periodic horizontal direction. Figure 14 depicts the curves $C_{P}$ and $S_{25}^{i}$ in $Y_{25}$. A zero next to a node of a stable surface represents a holomorphic node. Next to each $C_{P}$ are diagrams representing square tiled surfaces associated to each cusp of $W_{25}$ which meets $C_{P}$.

Projective structure of $Y_{D}$ So far, we have given $Y_{D}$ the structure of an algebraic variety and a complex orbifold. We can also give $Y_{D}$ the structure of a projective variety:

Proposition 8.9 $Y_{D}$ is a projective variety.

Proof Let $Z \subset \overline{\mathcal{M}}_{2}$ be the closure of the image of the natural map $X_{D} \rightarrow \overline{\mathcal{M}}_{2}$. The image of $\pi: Y_{D} \rightarrow \overline{\mathcal{M}}_{2}$ is contained in $Z$, so we have a finite surjective morphism $Y_{D} \rightarrow Z$. This means that $Y_{D}$ is the normalization of $Z$ in $K\left(Y_{D}\right)=K\left(X_{D}\right)$. By Theorem A.3, $Y_{D}$ is a projective variety because $\overline{\mathcal{M}}_{2}$ is projective. 


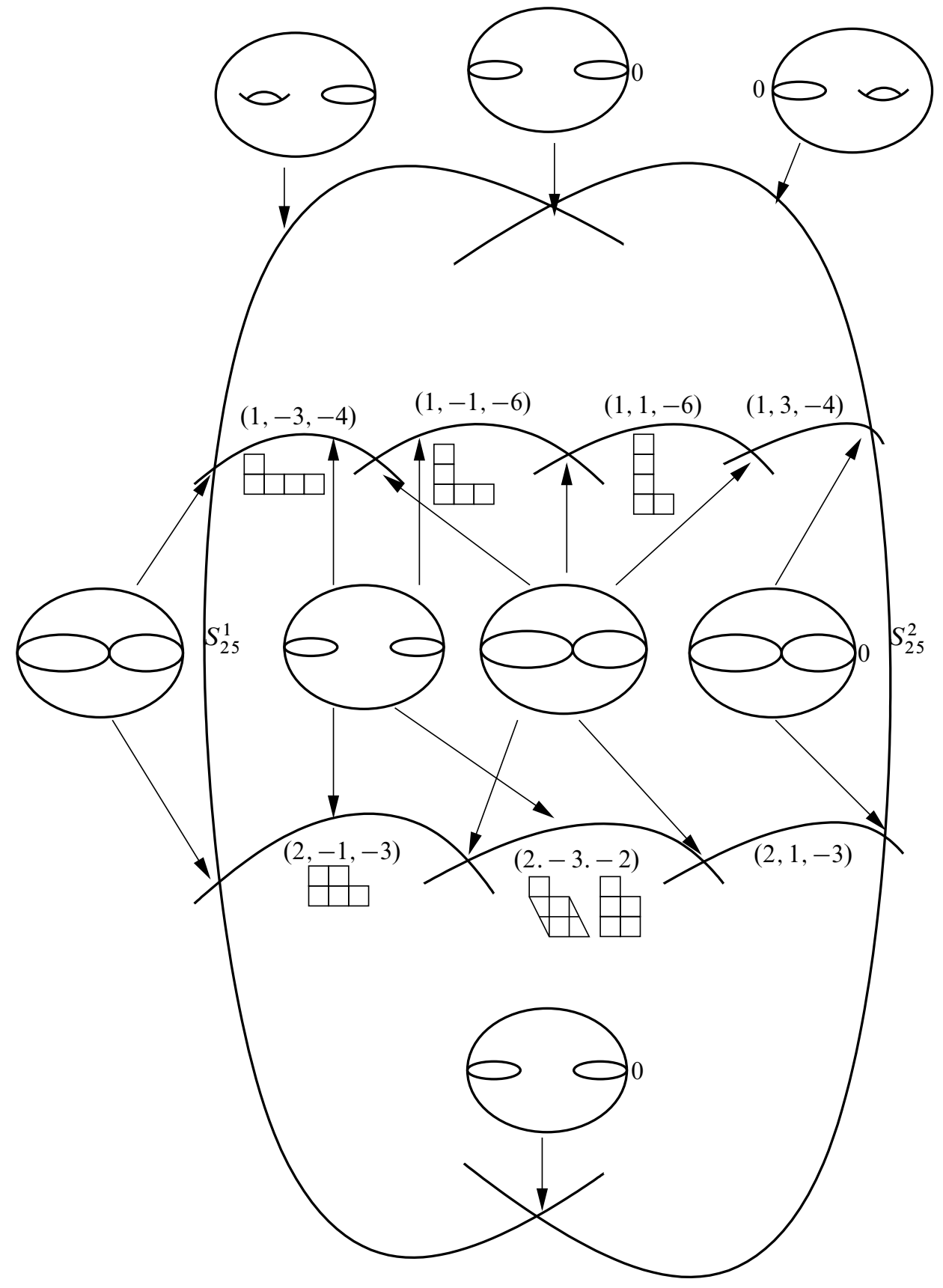

Figure 14: $Y_{25}$ 


\section{2 $Y_{D}$ maps to $\hat{X}_{D}$}

Recall that we introduced the Baily-Borel compactification $\hat{X}_{D}$ of $X_{D}$ in Section 2. If $D$ is not square, $\hat{X}_{D}$ consists of $X_{D}$ together with a finite set $C$ of cusps. If $D=d^{2}$, then $\hat{X}_{d^{2}}$ consists of $X_{D}$ together with the curves $R_{d^{2}}^{1}$ and $R_{d^{2}}^{2}$ isomorphic to $\mathbb{H} / \Gamma_{1}(d)$ and the finite set $C$ of cusps. Let $i: X_{D} \rightarrow Y_{D}$ be the natural inclusion.

Theorem 8.10 There is a unique morphism $p: Y_{D} \rightarrow \widehat{X}_{D}$ such that $p \circ i=\mathrm{id}_{X_{D}}$. This map $p$ has the following properties:

- $\quad p$ maps each curve $C_{P}$ onto a cusp of $\hat{X}_{D}$, and this induces a bijection between the connected components of $\bigcup_{P} C_{P}$ and the set of cusps $C \subset \hat{X}_{D}$.

- The restriction,

$$
p: Y_{D} \backslash \bigcup_{P} C_{P} \rightarrow X_{D} \backslash C
$$

is an isomorphism.

- $p$ maps $S_{d^{2}}^{i}$ isomorphically onto $R_{d^{2}}^{i}$.

Proof Recall from Section 2 that there is the natural morphism $j: \widehat{X}_{D} \rightarrow \widehat{\mathcal{A}}_{2}$ with image $Z$ such that $j: \hat{X}_{D} \rightarrow Z$ is the normalization of $Z$ in $K\left(X_{D}\right)$.

The varieties $Y_{D}$ and $\hat{X}_{D}$ both contain $X_{D}$ as an open dense set, so we can identify both of their function fields with $K\left(X_{D}\right)$. The morphism $q: Y_{D} \rightarrow \widehat{\mathcal{A}}_{2}$ which is the composition of the maps

$$
Y_{D} \rightarrow \overline{\mathcal{M}}_{2} \rightarrow \widehat{\mathcal{A}}_{2}
$$

has image $Z$. By the universal property of normalization, Theorem A.2, there is a map $p: Y_{D} \rightarrow \hat{X}_{D}$ such that $p^{*}$ is the identity on $K\left(X_{D}\right)$ and $j \circ p=q$. Since $p^{*}$ is the identity on $K\left(X_{D}\right)$, we must have $p \circ i=\operatorname{id}_{X_{D}}$.

The locus of points in $Y_{D}$ representing stable Riemann surfaces whose Jacobian is $\left(\mathbb{C}^{*}\right)^{2}$ is the union $\bigcup C_{P}$. Thus $q^{-1}\left(\mathcal{A}_{0}\right)=\bigcup C_{P}$. We have $j^{-1}\left(\mathcal{A}_{0}\right)=C$, so $p^{-1}(C)=\bigcup C_{P}$. By Theorem A.4, Zariski's Main Theorem, the fiber of $p$ over each point in $C$ is connected, so $p$ induces a bijection between the components of $\bigcup C_{P}$ and the points of $C$.

For each point $t \in \widehat{X}_{D} \backslash C$, the image $j(t)$ represents either an Abelian surface in $\mathcal{A}_{2}$ or an elliptic curve in $\mathcal{A}_{1}$ In the either case, there are only finitely many points in $Y_{D}$ which map to $j(t)$, so the fiber $p^{-1}(t)$ is finite. This fiber is also connected by Zariski's Main Theorem, so it is a single point. This means that the restriction,

$$
p: Y_{D} \backslash \bigcup_{P} C_{P} \rightarrow X_{D} \backslash C
$$


is a bijection, and it is thus an isomorphism because these are normal varieties.

The locus of points $t$ in $\hat{X}_{d^{2}}$ such that $j(t) \in \mathcal{A}_{1}$ is the union of $R_{d^{2}}^{1}$ and $R_{d^{2}}^{2}$, and the locus of points in $Y_{d^{2}}$ whose Jacobians are extensions of elliptic curves is the union of $S_{d^{2}}^{1}$ and $S_{d^{2}}^{2}$. This means that $p$ must take the curves $S_{d^{2}}^{i}$ to $R_{d^{2}}^{i}$, and the restriction of $p$ to the $S_{d^{2}}^{i}$ is an isomorphism onto the $R_{d^{2}}^{i}$ because $S_{d^{2}}^{i}$ is disjoint from each $C_{P}$.

Cohomology of $\boldsymbol{Y}_{\boldsymbol{D}}$ We can use Theorem 8.10 to get information about the cohomology of $Y_{D}$. In $H^{2}\left(Y_{D} ; \mathbb{Q}\right)$, let $B$ be the subspace generated by the fundamental classes of the rational curves $C_{P}$.

Theorem 8.11 The intersection form on $B$ is negative definite.

Proof Consider the map $q: Y_{D}^{\prime} \rightarrow Y_{D}$ obtained by resolving the singularities at the points $c_{P}$ of $Y_{D}$. Define $r: Y_{D}^{\prime} \rightarrow \widehat{X}_{D}$ by $r=p \circ q$, and let $E=r^{-1}(C)$. The locus $E$ is the union of irreducible curves $E_{i}$. By possibly taking some blow-ups of $Y_{D}^{\prime}$, we can assume that the curves $E_{i}$ are nonsingular and transverse (actually, performing these blow-ups is not necessary; see the remark below).

Let $B^{\prime} \subset H^{2}\left(Y_{D}^{\prime} ; \mathbb{Q}\right)$ be the subspace spanned by the fundamental classes of the $E_{i}$. By Theorem A.10, the intersection form on $B^{\prime}$ is negative definite.

The map $q^{*}: B \rightarrow B^{\prime}$ preserves the intersection forms on $B$ and $B^{\prime}$ because $q$ is degree one. We claim that $q^{*}: B \rightarrow B^{\prime}$ is injective. To see this, define a linear map $r: B^{\prime} \rightarrow B$ as follows. If $E_{i} \subset E$, and $q\left(E_{i}\right)$ is one of the curves $C_{P}$, let $r\left(\left[E_{i}\right]\right)$ be the fundamental class of that curve. Otherwise, $q\left(E_{i}\right)$ is a point; in this case let $r\left(\left[E_{i}\right]\right)=0$. We have $r \circ q^{*}=\mathrm{id}_{B}$ on $B$, so $q^{*}$ is injective on $B$.

Thus $q^{*}$ embeds $B$ in $B^{\prime}$, preserving the intersection forms. Since the intersection form on $B^{\prime}$ is negative definite, the intersection form on $B$ must be as well.

Remark We can actually describe the variety $Y_{D}^{\prime}$ above very explicitly. The exceptional fiber of a resolution of a cyclic quotient singularity consists of a chain of rational curves $\left\{E_{i}\right\}_{i=1}^{n}$ with $E_{i}$ having one transverse intersection with $E_{i+1}$ if $i \leq i \leq n-1$. This is described in [75].

Thus resolving each $c_{P} \in Y_{D}$ which is singular replaces each chain of curves $C_{P}$ with a longer chain of rational curves with transverse crossings.

Since $Y_{D}$ is an orbifold, Poincaré duality holds for rational cohomology, as Satake showed in [68]. This together with Theorem 8.11 means that we can define the orthogonal projection $\pi_{B}: H^{2}\left(Y_{D} ; \mathbb{Q}\right) \rightarrow B$. 
Corollary 8.12 If $D$ is not square, then

$$
\pi_{B}\left[\bar{P}_{D}\right]=\pi_{B}\left[\bar{W}_{D}\right]
$$

as classes in $H^{2}\left(Y_{D} ; \mathbb{Q}\right)$. If $D=d^{2}$, then

$$
\pi_{B}\left[\bar{P}_{d^{2}}\right]=\pi_{B}\left[\bar{W}_{d^{2}}\right]+\pi_{B}\left[\bar{S}_{d^{2}}^{2}\right]
$$

Proof For each curve $C_{P}$, the number of intersection points of $C_{P}$ with $\bar{P}_{D}$ is equal to the number of intersection points of $C_{P}$ with $\bar{W}_{D}$ and $\bar{S}_{D}^{2}$ by Part 4 of Theorem 8.7 (using the convention that $S_{D}^{2}$ is empty if $D$ is not square). Since these intersection points are transverse, this implies

$$
\left[C_{P}\right] \cdot\left[\bar{P}_{D}\right]=\left[C_{P}\right] \cdot\left(\left[\bar{W}_{D}\right]+\left[\bar{S}_{D}^{2}\right]\right) .
$$

The desired equations then follow because the intersection matrix of the $C_{P}$ is nondegenerate.

\subsection{Involution of $Y_{D}$}

We now study the extension of the involution $\tau$ of $X_{D}$ to $Y_{D}$. We then use this involution to deduce information about the fundamental classes of the curves $\bar{W}_{D}^{\epsilon}$.

Lemma 8.13 Let $\Gamma \subset X_{D} \times \overline{\mathcal{M}}_{2}$ be the graph of the natural map $X_{D} \rightarrow \overline{\mathcal{M}}_{2}$, and let $\bar{\Gamma}$ be the closure of $\Gamma$ in $\widehat{X}_{D} \times \overline{\mathcal{M}}_{2}$. Then there is a morphism $q: Y_{D} \rightarrow \bar{\Gamma}$ which is the normalization of $\bar{\Gamma}$.

Proof We have a map $q=p \times \pi: Y_{D} \rightarrow \hat{X}_{D} \times \overline{\mathcal{M}}_{2}$ by Theorem 8.10, and the image of $q$ is in $\bar{\Gamma}$. To show that $q: Y_{D} \rightarrow \bar{\Gamma}$ is the normalization of $\bar{\Gamma}$, we need only to show that $q$ is finite and birational. The map $q$ is birational because $p: Y_{D} \rightarrow \widehat{X}_{D}$ is birational, as is the projection $\bar{\Gamma} \rightarrow \hat{X}_{D}$. The map $q$ is finite because the map $\pi: Y_{D} \rightarrow \overline{\mathcal{M}}_{2}$ is finite.

Theorem 8.14 The involution $\tau$ of $X_{D}$ extends to an involution $\tau$ of $Y_{D}$. This involution satisfies

$$
\begin{gathered}
\tau\left(S_{D}^{1}\right)=S_{D}^{2}, \\
\tau\left(C_{P}\right)=C_{t(P)},
\end{gathered}
$$

where $t$ is the involution on the set of nondegenerate $Y_{D}$-prototypes defined in Section 3.1. 
Proof Let $\bar{\Gamma} \subset \widehat{X}_{D} \times \overline{\mathcal{M}}_{2}$ as in Lemma 8.13. The involution,

$$
\tilde{\tau}=\tau \times \operatorname{id}_{\overline{\mathcal{M}}_{2}}: \hat{X}_{D} \times \overline{\mathcal{M}}_{2} \rightarrow \hat{X}_{D} \times \overline{\mathcal{M}}_{2}
$$

restricts to an involution $\tilde{\tau}$ of $\bar{\Gamma}$. By the universal property, Theorem A.2, the composition $\tilde{\tau} \circ q: Y_{D} \rightarrow \bar{\Gamma}$ lifts to a map $\tau: Y_{D} \rightarrow Y_{D}$ which extends $\tau$ on $X_{D}$. Since $\tau^{2}$ is the identity on the open, dense subset $X_{D}$ of $Y_{D}$, the map $\tau$ is an involution of $Y_{D}$.

The involution $\tau$ of $\hat{X}_{D}$ sends $R_{D}^{1}$ to $R_{D}^{2}$. Since the map $p: Y_{D} \rightarrow X_{D}$ commutes with the involutions, we must have $\tau\left(S_{D}^{1}\right)=S_{D}^{2}$ by Theorem 8.10.

We are regarding $X_{D} \subset \mathbb{P} \Omega \overline{\mathcal{M}}_{2}$ as the set of stable Abelian differentials $(X,[\omega])$, where $X$ has a compact $\operatorname{Jacobian}$, and $\operatorname{Jac}(X)$ has real multiplication $\rho: \mathcal{O}_{D} \rightarrow \operatorname{End}(\operatorname{Jac}(X))$ with $\omega$ as an 1-eigenform defined up to constant multiple. In these terms, $\tau(X,[\omega])$ is the pair $\left(X,\left[\omega^{\prime}\right]\right)$, where $\omega^{\prime}$ is an 2-eigenform for the same real multiplication on $\operatorname{Jac}(X)\left(\omega^{\prime}\right.$ is then an 1 -eigenform for the Galois conjugate real multiplication $\left.\rho^{\prime}\right)$.

Now suppose that $(X,[\omega]) \in X_{D}$ is close to a point in the curve $C_{P}^{0}$, and $\left(X,\left[\omega^{\prime}\right]\right)=$ $\tau(X,[\omega])$ is close to a point in $C_{P^{\prime}}^{0}$. We must show that $P^{\prime}=t(P)$.

Recall the definition of the prototype $P$ from Section 7.6. On $(X, \omega)$, there are two tall cylinders $C_{1}$ and $C_{2}$. Let $\left\{\alpha_{i}, \beta_{i}\right\}_{i=1}^{2}$ be a symplectic basis of $H_{1}(X ; \mathbb{Z})$ such that $\alpha_{i}$ represents the cylinder $C_{i}$ and $\omega\left(\alpha_{2}\right) / \omega\left(\alpha_{1}\right) \geq 1$. Let $\mu \in K_{D}$ be such that $\mu \cdot \alpha_{1}=\alpha_{2}$, and let $\psi(t)=a t^{2}+b t+c$ be as in Proposition 3.4, in particular $\psi(\mu)=0$. For $\lambda \in K_{D}$, let $T_{\lambda}$ be the matrix of the action of $\lambda$ on $H_{1}(X ; \mathbb{Q})$ in the symplectic basis $\left\{\alpha_{i}, \beta_{i}\right\}$. Then $T_{a \mu}$ is as in (7-9). The prototype is then $P=(a, b, c, \bar{q})$, where $a$, $b$, and $c$ are as above, and $\bar{q}$ is the reduction of the upper right entry of $T_{a \mu}$ modulo $\operatorname{gcd}(a, b, c)$.

There are two cases to consider. First suppose that $\mu^{(2)} \leq-1$, which is equivalent to $a-b+c \leq 0$. Let $\left\{\alpha_{i}^{\prime}, \beta_{i}^{\prime}\right\}_{i=1}^{2}$ be the symplectic basis,

$$
\left\{\alpha_{1}^{\prime}, \alpha_{2}^{\prime}, \beta_{1}^{\prime}, \beta_{2}^{\prime}\right\}=\left\{\alpha_{1},-\alpha_{2}, \beta_{1},-\beta_{2}\right\},
$$

of $H_{1}(X ; \mathbb{Z})$. Let $\tilde{\mu}=-\mu$, and normalize $\left[\omega^{\prime}\right]$ so that $\omega^{\prime}\left(\alpha_{1}^{\prime}\right)=1$. Then $\tilde{\mu} \cdot \alpha_{1}^{\prime}=\alpha_{2}^{\prime}$, and

$$
\omega^{\prime}\left(\alpha_{2}^{\prime}\right)=\omega^{\prime}\left(\tilde{\mu} \cdot \alpha_{1}^{\prime}\right)=\tilde{\mu}^{(2)} \omega^{\prime}\left(\alpha_{1}^{\prime}\right)=-\mu^{(2)} \geq 1 .
$$

This means that we can use the symplectic basis $\left\{\alpha_{i}^{\prime}, \beta_{i}^{\prime}\right\}$ to calculate $P^{\prime}$. If $\psi^{\prime}(t)=$ $a^{\prime} t^{2}+b^{\prime} t+c^{\prime}$ is the polynomial from Proposition 3.4 with $\psi^{\prime}(\tilde{\mu})=0$, then

$$
\psi^{\prime}(t)=\psi(-t)=a t^{2}-b t+c,
$$


so $\left(a^{\prime}, b^{\prime}, c^{\prime}\right)=(a,-b, c)$. For $\lambda \in K_{D}$, let $T_{\lambda}^{\prime}$ be the matrix $T_{\lambda}$ in the new basis $\left\{\alpha_{i}, \beta_{i}\right\}$. Then a simple calculation shows

$$
T_{a^{\prime} \tilde{\mu}}^{\prime}=T_{-a \mu}^{\prime}=\left(\begin{array}{rrrr}
0 & -c & 0 & q \\
a & b & -q & 0 \\
0 & 0 & 0 & a \\
0 & 0 & -c & b
\end{array}\right) .
$$

Thus $P^{\prime}=(a,-b, c, \bar{q})=t(P)$.

Now suppose that $-1<\mu^{(2)}<0$, which is equivalent to $a-b+c>0$. Let $\left\{\alpha_{i}^{\prime}, \beta_{i}^{\prime}\right\}_{i=1}^{2}$ be the symplectic basis,

$$
\left\{\alpha_{1}^{\prime}, \alpha_{2}^{\prime}, \beta_{1}^{\prime}, \beta_{2}^{\prime}\right\}=\left\{\alpha_{2},-\alpha_{1}, \beta_{2},-\beta_{1}\right\},
$$

of $H_{1}(X ; \mathbb{Z})$, let $\tilde{\mu}=-1 / \mu$, and normalize $\omega^{\prime}$ so that $\omega^{\prime}\left(\alpha_{1}^{\prime}\right)=1$. Then $\tilde{\mu} \cdot \alpha_{1}^{\prime}=\alpha_{2}^{\prime}$, and

$$
\omega^{\prime}\left(\alpha_{2}^{\prime}\right)=-\mu^{(2)}>1
$$

as before. If $\psi^{\prime}(t)=a^{\prime} t^{2}+b^{\prime} t+c^{\prime}$ is the polynomial from Proposition 3.4 with $\psi^{\prime}(\tilde{\mu})=0$, then

$$
\psi^{\prime}(t)=-t^{2} \psi(-1 / t)=-c t^{2}+b t-a,
$$

so $\left(a^{\prime}, b^{\prime}, c^{\prime}\right)=(-c, b,-a)$. For $\lambda \in K_{D}$, let $T_{\lambda}^{\prime}$ be the matrix $T_{\lambda}$ in the new basis $\left\{\alpha_{i}, \beta_{i}\right\}$. Then,

$$
T_{a^{\prime} \tilde{\mu}}=T_{c / \mu}=a c\left(T_{a \mu}\right)^{-1}=\left(\begin{array}{rrrr}
-b & c & 0 & q \\
-a & 0 & -q & 0 \\
0 & 0 & -b & -a \\
0 & 0 & c & 0
\end{array}\right),
$$

and in the new basis,

$$
T_{a^{\prime} \tilde{\mu}}^{\prime}=\left(\begin{array}{rrrr}
0 & a & 0 & q \\
-c & -b & -q & 0 \\
0 & 0 & 0 & -c \\
0 & 0 & a & -b
\end{array}\right)
$$

Thus $P^{\prime}=(-c, b,-a, \bar{q})=t(P)$.

It is not true that $\tau\left(W_{D}^{0}\right)=W_{D}^{1}$, but we will prove that something like this is true on the level of cusps.

Theorem 8.15 Suppose $D \equiv 1(\bmod 8)$. If $D$ is not square, then for any nondegenerate $Y_{D}$-prototype,

$$
\#\left(\bar{W}_{D}^{1} \cap C_{P}\right)=\#\left(\bar{W}_{D}^{0} \cap \tau\left(C_{P}\right)\right) .
$$


If $D=d^{2}$, then

$$
\#\left(\bar{W}_{d^{2}}^{1} \cap C_{P}\right)+\#\left(\bar{S}_{d^{2}}^{2} \cap C_{P}\right)=\#\left(\bar{W}_{d^{2}}^{0} \cap \tau\left(C_{P}\right)\right) .
$$

Proof Suppose $P$ is a nondegenerate, nonterminal, noninitial $Y_{D}$-prototype. Recall that the cusps of $W_{D}$ lying on $C_{P}$, where $P=(a, b, c, \bar{q})$, are the points $w_{Q}$, where $Q=(a, b, c, \bar{r})$ is a $W_{D}$-prototype with $\bar{r} \in \mathbb{Z} / \operatorname{gcd}(a, c)$, and $\bar{r} \equiv q$ $(\bmod \operatorname{gcd}(a, b, c))$. The cusp $w_{Q}$ lies on $W_{D}^{\epsilon(Q)}$, where $\epsilon(Q)$ is given by (4-6).

There are two cases to consider, depending on whether or not $a-b+c \leq 0$. First suppose $a-b+c \leq 0$. In this case,

$$
\epsilon(t(Q)) \equiv \epsilon(Q)+b \equiv \epsilon(Q)+1 \quad(\bmod 2)
$$

for any $W_{D}$-prototype $Q$ associated to $P$, using the fact that $b$ is odd because $b^{2}-4 a c=D \equiv 1(\bmod 8)$. It follows that $C_{P}$ has as many cusps of $W_{D}^{1}$ as $C_{t(P)}$ has cusps of $W_{D}^{0}$.

Now suppose that $a-b+c>0$. Here there are four cases to consider, depending on the parity of $a$ and $c$. First suppose $a \equiv 1(\bmod 2)$, and $c \equiv 0(\bmod 2)$. In this case, for any $W_{D}$-prototype $Q$ associated to $P$, we have $\epsilon(Q) \equiv 0(\bmod 2)$ and $\epsilon(t(Q)) \equiv 1(\bmod 2)$. Thus, every cusp of $W_{D}$ on $C_{P}$ is in $W_{D}^{0}$, and $C_{t(P)}$ has the same number of cusps, all in $W_{D}^{1}$.

Suppose $a \equiv 0(\bmod 2)$, and $c \equiv 1(\bmod 2)$. In this case, for any $W_{D}$-prototype $Q$ associated to $P$, we have $\epsilon(Q) \equiv 1(\bmod 2)$ and $\epsilon(t(Q)) \equiv 0(\bmod 2)$. Thus, every cusp of $W_{D}$ on $C_{P}$ is in $W_{D}^{1}$, and $C_{t(P)}$ has the same number of cusps, all in $W_{D}^{0}$.

Suppose $a \equiv c \equiv 0(\bmod 2)$. In this case, for any $W_{D}$-prototype $Q=(a, b, c, \bar{r})$ associated to $P$, we have $\epsilon(Q) \equiv \epsilon(t(Q)) \bar{r}(\bmod 2)$. Since $\operatorname{gcd}(a, b, c) \equiv 1(\bmod 2)$, this means that exactly half of the cusps of $W_{D}$ on $C_{P}$ are on $W_{D}^{1}$, and the same is true for $C_{t(P)}$.

The last case, $a \equiv c \equiv 1(\bmod 2)$ doesn't occur because $b^{2}-4 a c \equiv 1(\bmod 8)$. Thus we have shown (8-4).

It remains to prove (8-5) when $P$ is an initial or terminal prototype. We claim that if $P$ is an initial $Y_{d^{2}}$-prototype, then $C_{P}$ intersects $\bar{W}_{d^{2}}^{0}$ once and is disjoint from $\bar{W}_{d^{2}}^{1}$. Let $P=(a, b, c, \bar{q})$ be an initial prototype. Since $a-b+c=0$,

$$
\operatorname{gcd}(a, c)=\operatorname{gcd}(a, b, c)
$$


so $\operatorname{mult}(P)=1$, and $\bar{W}_{d^{2}}$ intersects $C_{P}$ once. We have

$$
\begin{aligned}
& b=a+c \\
& \Rightarrow(a+c)^{2}-4 a c=d^{2} \\
& \Rightarrow a-c=d \\
& \Rightarrow a \equiv c+1 \quad(\bmod 2),
\end{aligned}
$$

and furthermore $f=d$. It follows that $\epsilon(P)=0$, thus the intersection point of $C_{P}$ with $\bar{W}_{d^{2}}$ is in $\bar{W}_{d^{2}}^{0}$.

We saw in Theorem 8.7 that if $P$ is a terminal $Y_{D}$-prototype, then $C_{P}$ intersects $\bar{S}_{d^{2}}^{2}$ once and is disjoint from $\bar{W}_{d^{2}}$. Thus if $P$ is a terminal prototype, then both sides of $(8-5)$ are one, and if $P$ is an initial prototype, then both sides of (8-5) are zero.

Corollary 8.16 If $D$ is not square, then

$$
\tau_{*} \pi_{B}\left[\bar{W}_{D}^{0}\right]=\pi_{B}\left[\bar{W}_{D}^{1}\right]
$$

in $H^{2}\left(Y_{D} ; \mathbb{Q}\right)$. If $D=d^{2}$, then

$$
\tau_{*} \pi_{B}\left[\bar{W}_{d^{2}}^{0}\right]=\pi_{B}\left[\bar{W}_{d^{2}}^{1}\right]+\pi_{B}\left[\bar{S}_{d^{2}}^{2}\right]
$$

in $H^{2}\left(Y_{d^{2}} ; \mathbb{Q}\right)$.

Proof Since the intersection pairing on $B \subset H^{2}\left(Y_{D} ; \mathbb{Q}\right)$ is nondegenerate by Theorem 8.11 , it suffices to show that

$$
\begin{aligned}
{\left[C_{P}\right] \cdot\left(\tau_{*} \pi_{B}\left[\bar{W}_{D}^{0}\right]\right) } & =\left[C_{P}\right] \cdot \pi_{B}\left[\bar{W}_{D}^{1}\right], \\
\text { and } \quad & {\left[C_{P}\right] \cdot\left(\tau_{*} \pi_{B}\left[\bar{W}_{d^{2}}^{0}\right]\right)=\left[C_{P}\right] \cdot\left(\pi_{B}\left[\bar{W}_{d^{2}}^{1}\right]+\pi_{B}\left[\bar{S}_{d^{2}}^{2}\right]\right) }
\end{aligned}
$$

for each $C_{P}$. Since the intersections of $\bar{W}_{D}$ and $\bar{S}_{d^{2}}^{2}$ with each $C_{P}$ are transverse, this follows directly from Theorem 8.15.

\section{Line bundles over $Y_{D}$}

In this section, we will define extensions of some line bundles over $X_{D}$ to $Y_{D}$, and we will calculate their Chern classes. In Section 9.1, we discuss background material on vector bundles over orbifolds. In Section 9.2, we recall Mumford's notion of a good Hermitian metric, which allows one to express the Chern classes of a vector bundle in terms of curvatures of singular Hermitian metrics. In Section 9.3, we discuss the extension of $\Omega X_{D}$ to $Y_{D}$, and we discuss the extension of $T^{*} \mathcal{A}_{D}$ to $Y_{D}$ in Section 9.4. 


\subsection{Orbifold vector bundles}

Most of the theory of vector bundles over manifolds works for vector bundles over orbifolds. Here following Chen and Ruan [18] we discuss the facts that we need and refer the reader to the Appendix to [18] for details.

Let $X$ be a complex orbifold with an atlas $\left\{U_{\alpha} / G_{\alpha} \rightarrow X\right\}$, where $U_{\alpha} \subset \mathbb{C}^{n}$, and $G_{\alpha}$ is a finite automorphism group of $U_{\alpha}$ (we allow $G_{\alpha}$ to have elements which act trivially on $U_{\alpha}$, and $X$ is said to be reduced if no such element exists). A rank $r$ holomorphic orbifold bundle over $X$ is an orbifold $E$ with a map $\pi: E \rightarrow X$ such that for each $\alpha$,

$$
\pi^{-1}\left(U_{\alpha}\right) \cong\left(U_{\alpha} \times \mathbb{C}^{r}\right) / G_{\alpha},
$$

where $G_{\alpha}$ acts on $U_{\alpha} \times \mathbb{C}^{r}$ by

$$
g \cdot(z, v)=(g \cdot z, \rho(z, g)(v))
$$

for some $\rho: U_{\alpha} \times G_{\alpha} \rightarrow \mathrm{GL}_{r} \mathbb{C}$ which is a homomorphism if we fix $z$ and is holomorphic if we fix $g$.

Nonreduced orbifold structures occur naturally. For example, $\mathcal{M}_{2}$ has a nonreduced orbifold structure, where the local group $G$ at a point $X$ is $\operatorname{Aut}(X)$. This is nonreduced because the hyperelliptic involution $J$ acts trivially. The associated reduced orbifold structure has local group $G=\operatorname{Aut}(X) / J$ at $X$. With this definition of an orbifold vector bundle, $\Omega \mathcal{M}_{2}$ is only an orbifold vector bundle if we take the nonreduced orbifold structure on $\mathcal{M}_{2}$. When necessary, we will implicitly use this orbifold structure. Similarly, $X_{D}$ has a nonreduced orbifold structure with local group $G=\operatorname{Aut}(A, \rho)$ at $(A, \rho)$.

We want to be able to pull back bundles along maps between orbifolds. The operation of pulling back a bundle is much more complicated in the category of orbifolds than in the category of topological spaces. In particular, given a map $f: X \rightarrow Y$ of orbifolds, it is not always possible to pull back a bundle over $Y$ by $f$; however, this is possible if $f$ is what is called a good map. We will informally discuss pullbacks of orbifold bundles without actually defining a good map.

Given a map $f: X \rightarrow Y$ of orbifolds and charts $V / H \subset Y$ and $U / G \subset X$ such that $f(U / G) \subset V / H$, there is a lift $\tilde{f}: U \rightarrow V$ such that for each $g \in G$ there is some $h \in H$ such that

$$
\tilde{f}(g \cdot z)=h \cdot \tilde{f}(z) .
$$

Note that there may not be a group homomorphism $\sigma: G \rightarrow H$ such that

$$
\tilde{f}(g \cdot z)=\sigma(g) \tilde{f}(z) .
$$


If $f$ is good in the sense of [18], then such a homomorphism does exist. The actual definition of a good map is some complicated global condition which locally amounts to the existence of such a homomorphism; we will not get into this here.

In the notation of the previous paragraph, suppose that $f: U / G \rightarrow V / H$ is good and we have over $V / H$ a vector bundle $E=\left(V \times \mathbb{C}^{r}\right) / H \rightarrow V / H$, with $H$ acting on $V \times \mathbb{C}^{r}$ by $\rho: V \times H \rightarrow \mathrm{GL}_{r} \mathbb{C}$. We define $f^{*} E$ over $U$ by

$$
f^{*} E=\left(U \times \mathbb{C}^{r}\right) / G
$$

where $G$ acts on $U \times \mathbb{C}^{r}$ by $g \cdot(z, v)=(g \cdot z, \tau(z, g)(v))$, with

$$
\tau(z, g)=\rho(\tilde{f}(z), \sigma(g)) .
$$

Globally, the pullback of a bundle $E \rightarrow Y$ along a map $f: X \rightarrow Y$ is an orbifold vector bundle $f^{*} E$ which locally satisfies (9-1).

Given an orbifold $X$, write $X_{\text {reg }}$ for the set of $p$ points of $X$ such that for a chart $U / G \ni p$, no element of $G$ fixes $p$. The following theorem follows from Lemmas 4.4.3 and 4.4.11 of [18].

Theorem 9.1 Let $f: X \rightarrow Y$ be a map of orbifolds such that $f^{-1}\left(Y_{\text {reg }}\right)$ is an open, dense, and connected subset of $X$. Then $f$ is a good map of orbifolds, and for any orbifold vector bundle $E \rightarrow Y$, there is a well-defined pullback bundle $f^{*} E \rightarrow X$. The pullback bundle satisfies the naturality property

$$
c_{1}\left(f^{*} E\right)=f^{*} c_{1}(E) .
$$

Remark Chern classes for orbifold vector bundles are defined using the Chern-Weil construction, which associates a de Rham cohomology class to a metric on an orbifold bundle. This theory is worked out in [18].

\subsection{Good metrics}

It is well known that the Chern classes of a complex vector bundle can be given in terms of the curvature of a Hermitian metric. In [63], Mumford showed that for a Hermitian metric with certain mild singularities, called a good metric, the Chern classes of the vector bundle can still be given in terms of the curvature of the metric. Mumford's results are in terms of nonsingular complex projective varieties, but his results and proofs work just as well in the setting of complex orbifolds. In this section, following [63], we will summarize what we need about good metrics, translating to the setting of orbifolds. 
Let $X \subset \bar{X}$ be complex $n$-dimensional orbifolds with $D=\bar{X}-X$ a divisor and $\bar{X}$ compact. Suppose that we can cover $D$ with coordinate charts $\Delta^{n} / G$, where $\Delta^{n} \subset \mathbb{C}^{n}$ is a polydisk, and $G$ is a finite group of automorphisms of $\Delta^{n}$ such that:

- Each transformation $g \in G$ is of the form,

$$
g\left(z_{1}, \ldots, z_{n}\right)=\left(\theta_{1} z_{1}, \ldots, \theta_{n} z_{n}\right),
$$

for some roots of unity $\theta_{i}$.

- $D \cap \Delta$ is a union of the coordinate axes $z_{1}=0, \ldots, z_{r}=0$ for $1 \leq r \leq n$.

In such a neighborhood $\Delta^{n} / G$, we have $\Delta^{n} \cap X=\left(\Delta^{*}\right)^{r} \times \Delta^{n-r}$. We give $\Delta^{n} \cap X$ a metric by putting the Poincaré metric,

$$
d s^{2}=\frac{|d z|^{2}}{|z|^{2}(\log |z|)^{2}},
$$

on the $\Delta^{*}$ factors and putting the Euclidean metric $|d z|^{2}$ on the $\Delta$ factors. Call the product metric $\omega$. This metric is $G$-invariant, so it is a metric on $\Delta^{n} / G$ in the orbifold sense.

Definition A $p$-form $\eta$ on $X$ has Poincaré growth if there is a cover of $\bar{X} \backslash X$ by polycylinders $\Delta_{\alpha}^{n} / G_{\alpha}$ as above such that in each $\Delta_{\alpha}^{n}$ we have some constant $C_{\alpha}$ such that for any $p$ vectors $t_{i}$ at any point $x \in \Delta_{\alpha}^{n} \cap X$,

$$
\left|\eta\left(t_{1}, \ldots, t_{p}\right)\right|^{2}<C_{\alpha} \omega_{\alpha}\left(t_{1}, t_{1}\right) \cdots \omega_{\alpha}\left(t_{p}, t_{p}\right),
$$

where $\omega_{\alpha}$ is the metric on $\Delta_{\alpha}^{n} / G_{\alpha}$ defined above.

In this paper, given a differential form $\eta$, we will write $\langle\eta\rangle$ for the current defined by $\eta$, and $[\eta]$ for the de Rham cohomology class defined by $\eta$.

Proposition 9.2 A $p$-form $\eta$ with Poincaré growth satisfies

$$
\int_{X}|\eta \wedge \zeta|<\infty
$$

for any smooth form $\zeta$ on $\bar{X}$ of complementary dimension. Thus, $\eta$ defines a $p$-current $\langle\eta\rangle$.

Definition A $p$-form $\eta$ in $X$ is $\operatorname{good}$ if $\eta$ and $d \eta$ both have Poincaré growth.

Proposition 9.3 If $\eta$ is a good $p$-form, then

$$
d\langle\eta\rangle=\langle d \eta\rangle .
$$


Note that it if $\eta$ is a closed good form, it is not necessarily true that $\eta=d \zeta$ for a good form $\zeta$; however, this will be the case if $\eta$ is the Chern form of a good Hermitian metric.

Let $\bar{L}$ be a holomorphic orbifold line bundle over $\bar{X}$ with $L$ the restriction to $X$, and let $H$ be a Hermitian metric on $E$.

Definition $H$ is a good metric if for every point $x \in D$, and every polycylindrical neighborhood $\Delta^{n} / G$ as described above with $D$ given by

$$
\prod_{i=1}^{k} z_{i}
$$

and for every holomorphic section $e$ of $\bar{E}$ over $\Delta^{n} / G$, setting $h=H(e, e)$, we have the following:

- For some $C>0$ and $m>1$,

$$
|h|,|h|^{-1}<C\left(\sum_{i=1}^{k} \log \left|z_{i}\right|\right)^{2 m} .
$$

- The 1 -forms $(\partial h) / h$ are $\operatorname{good}$ on $\Delta^{n} / G \cap X$.

Theorem 9.4 If $H$ is a good metric, then the Chern form $c_{1}(E, H)$ is good and the current $\left\langle c_{1}(E, H)\right\rangle$ represents the Chern class $c_{1}(\bar{E})$ in $H^{2}(\bar{X} ; \mathbb{Q})$. Furthermore,

$$
c_{1}(E, H)-d \eta
$$

is a smooth 2-form on $\bar{X}$ for some good 1-form $\eta$.

The last statement follows from the proof of Theorem 1.4 in [63].

We now show that Chern forms of good metrics behave as they should with respect to the cup product pairing and integrating over curves.

Proposition 9.5 Suppose $\operatorname{dim}_{\mathbb{C}} \bar{X}=2$. If $\omega$ and $\eta$ are Chern forms of two good metrics, then

$$
\langle[\omega],[\eta]\rangle=\int_{X} \omega \wedge \eta
$$


Proof By Theorem 9.4, we can write

$$
\begin{gathered}
\omega=\omega^{\prime}+d \omega^{\prime \prime} \\
\eta=\eta^{\prime}+d \eta^{\prime \prime},
\end{gathered}
$$

where $\omega^{\prime}$ and $\eta^{\prime}$ are smooth, closed 2-forms on $\bar{X}$, and $\omega^{\prime \prime}$ and $\eta^{\prime \prime}$ are good 1-forms. We have

$$
\begin{aligned}
\langle[\omega],[\eta]\rangle & =\int_{\bar{X}} \omega^{\prime} \wedge \eta^{\prime} \\
& =\int_{X}\left(\omega \wedge \eta-\omega \wedge d \eta^{\prime \prime}-d \omega^{\prime \prime} \wedge \eta+d \omega^{\prime \prime} \wedge d \eta^{\prime \prime}\right) \\
& =\int_{X}\left(\omega \wedge \eta+d\left(\omega \wedge \eta^{\prime \prime}\right)-d\left(\omega^{\prime \prime} \wedge \eta\right)+d\left(\omega^{\prime \prime} \wedge d \eta^{\prime \prime}\right)\right) .
\end{aligned}
$$

We claim that $\int_{X} d \alpha=0$ for any good 3-form $\alpha$. The last three terms in (9-4) are of this form, so (9-3) follows from this claim. By Proposition 9.3,

$$
\int_{X} d \alpha=\langle d \alpha\rangle(1)=d\langle\alpha\rangle(1)=-\langle\alpha\rangle(d 1)=0,
$$

so the claim follows.

Proposition 9.6 Suppose $C \subset \bar{X}$ is a curve with no irreducible component contained in $D$, and suppose $\omega$ is the Chern form of a good Hermitian metric. Then

$$
\langle[C],[\omega]\rangle=\int_{C} \omega .
$$

Sketch of proof Let $\omega=\omega^{\prime}+d \omega^{\prime \prime}$ with $\omega^{\prime}$ a smooth 2-form on $\bar{X}$ and $\omega^{\prime \prime}$ a good 1 -form. We have

$$
\langle[C],[\omega]\rangle=\int_{C} \omega^{\prime}=\int_{C} \omega-\int_{C} d \omega^{\prime \prime} .
$$

We need to show that the following two conditions hold:

$$
\begin{gathered}
\int_{C}|\omega|<\infty \\
\int_{C} d \omega^{\prime \prime}=0 .
\end{gathered}
$$

Let $C_{0}=C \backslash C \cap D$. We can speak of Poincaré growth for forms on $C_{0}$ using the compactification of $C_{0}$ obtained by adding a point to each cusp of $C_{0}$. It follows from the Schwartz Lemma that any good $p$-form on $X$ restricts to a good $p$-form on $C_{0}$, so $\left.\omega\right|_{C_{0}}$ and $\left.\omega^{\prime \prime}\right|_{C_{0}}$ are good. Then (9-6) follows from Proposition 9.2, and (9-7) follows from (9-5). 


\subsection{Extension of the bundle $\Omega X_{D}$ to $Y_{D}$}

Extension of $\boldsymbol{\Omega} \boldsymbol{X}_{\boldsymbol{D}}$ Recall that we have the map $\pi: Y_{D} \rightarrow \mathbb{P} \Omega \overline{\mathcal{M}}_{2}$. Let

$$
\pi_{1}: \mathbb{P} \Omega \overline{\mathcal{M}}_{2} \rightarrow \overline{\mathcal{M}}_{2}
$$

be the natural projection, and let

$$
\pi_{2}=\pi_{1} \circ \pi: Y_{D} \rightarrow \overline{\mathcal{M}}_{2} .
$$

Let $\Omega^{0} Y_{D}=\pi_{2}^{*}\left(\Omega \overline{\mathcal{M}}_{2}\right)$. It does not follow directly from Theorem 9.1 that this pullback is well defined because we must take the nonreduced orbifold structure on $\overline{\mathcal{M}}_{2}$ for $\Omega \overline{\mathcal{M}}_{2}$ to be a bundle, and then $\pi_{2}^{-1}\left(\overline{\mathcal{M}}_{2}\right)_{\text {reg }}=Y_{D}$. Instead, first pull back the square $\left(\Omega \overline{\mathcal{M}}_{2}\right)^{\otimes 2}$. This is a bundle over $\overline{\mathcal{M}}_{2}$ with the usual reduced orbifold structure, so $\pi_{2}^{-1}\left(\overline{\mathcal{M}}_{2}\right)_{\text {reg }}$ consists of the elliptic points of $X_{D}$ together with the elliptic points of $S_{d^{2}}^{i}$ and the curves $C_{P}$. This is an open, dense, and connected subset of $Y_{D}$, thus the pullback of the square is well defined by Theorem 9.1. Then define $\Omega^{0} Y_{D}$ to be the quotient of this pullback by \pm 1 .

Given $p \in Y_{D}$ with $\pi_{2}(p)=X \in \overline{\mathcal{M}}_{2}$, the fiber of $\Omega^{0} Y_{D}$ over $p$ is isomorphic to $\Omega(X) / G_{p}$ for some finite group $G_{p}$. If $p$ is not an orbifold point of $Y_{D}$, then $G=\{ \pm 1\}$. If $p$ is an elliptic point of $X_{D}$ or $S_{D}^{i}$, then $G_{p}=\operatorname{Aut}(X)$. If $p=c_{P}$ with $P$ a nondegenerate, nonterminal $Y_{D}$-prototype, then a priori $G_{p}$ could be bigger than $\{ \pm 1\}$ because $c_{P}$ is a singular point of $Y_{D}$. In fact, $\pi\left(c_{P}\right)$ is a nonsingular point of $\mathbb{P} \Omega \overline{\mathcal{M}}_{2}$ because the residues at the nodes of $\pi\left(c_{P}\right)$ are all different, so by (9-1), $G_{p} \cong\{ \pm 1\}$. Thus we have shown that the fiber of $\Omega^{0} Y_{D}$ over $p$ is $\Omega(X) /\{ \pm 1\}$ unless $p$ is an elliptic point of $X_{D}$ or $S_{D}^{i}$.

Over $\mathbb{P} \Omega \overline{\mathcal{M}}_{2}$, there is the canonical orbifold line bundle $\mathcal{O}(-1) \rightarrow \mathbb{P} \Omega \overline{\mathcal{M}}_{2}$ whose fiber over $(X,[\omega])$ is the subspace of $\Omega(X)$ spanned by $\omega$. Define

$$
\begin{aligned}
& \Omega^{1} Y_{D}=\pi^{*} \mathcal{O}(-1) \\
& \Omega^{2} Y_{D}=\tau^{*} \Omega^{1} Y_{D} .
\end{aligned}
$$

Since $\mathcal{O}(1) \subset \pi_{1}^{*} \Omega \overline{\mathcal{M}}_{2}$ as a sub line bundle, $\Omega^{1} Y_{D}$ is a sub line bundle of $\Omega^{0} Y_{D}$. The involution $\tau$ lifts to an involution of $\Omega^{0} Y_{D}$, so we can also regard $\Omega^{2} Y_{D}$ as a sub line bundle of $\Omega^{0} Y_{D}$. Given $p \in Y_{D}$ with $\pi_{2}(p)=X$, the $\operatorname{Jacobian} \operatorname{Jac}(X)$ comes with a choice of real multiplication by $\mathcal{O}_{D}$, and we can regard the fiber of $\Omega^{i} Y_{D}$ over $p$ as $\Omega^{i}(X)$, the space of $i$-eigenforms. Since

$$
\Omega(X)=\Omega^{1}(X) \oplus \Omega^{2}(X),
$$


we obtain

$$
\Omega^{0} Y_{D}=\Omega^{1} Y_{D} \oplus \Omega^{2} Y_{D}
$$

Define

$$
Q^{i} Y_{D}=\left(\Omega^{i} Y_{D}\right)^{2},
$$

which parameterizes quadratic differentials which are squares of $i$-eigenforms. In what follows, we will sometimes abbreviate $\Omega^{1} Y_{D}$ and $Q^{1} Y_{D}$ by $\Omega Y_{D}$ and $Q Y_{D}$.

Proposition 9.7 The restriction of $Q Y_{D}$ to each curve $C_{P} \subset Y_{D}$ is trivial.

Sketch of proof Define a global nonzero section of $Q Y_{D}$ over $C_{P}$ as follows. If $X \in C_{P}^{0}$, then let $q_{X} \in Q(X)$ be the unique quadratic differential which is the square of the Abelian differential $\omega_{X} \in \Omega(X)$ which has residue 1 at both nonseparating nodes of $X$. Otherwise, $X$ has three nonseparating nodes. In this case, define $q_{X}$ in the same way, using the two nodes of $X$ which are limits of nodes of surfaces in $C_{P}^{0}$.

Chern class of $\Omega Y_{D}$ In Section 2.3, we gave $\Omega X_{D}$ a Hermitian metric $h_{\Omega}$. In terms of Riemann surfaces, the metric is given on the fiber over $X$ by

$$
h_{\Omega}(\omega, \omega)=\int_{X}|\omega|^{2} .
$$

The induced metric $h_{Q}$ on $Q X_{D}$ is given in the fiber over $X$ by

$$
h_{Q}(q, q)=\left(\int_{X}|q|\right)^{2} .
$$

On $Y_{D}$, these metrics become singular along the curves $C_{P}$ and $\bar{S}_{D}^{1}$ because stable Abelian differentials representing points on these curves have infinite area.

Theorem 9.8 The metric $h_{Q}$ on $Q Y_{D}$ is a good metric.

Lemma 9.9 Let $H$ be a singular Hermitian metric on the trivial bundle $L=\Delta^{n} \times \mathbb{C}$ over the polydisk $\Delta^{n} \in \mathbb{C}^{n}$, and let $s$ be a holomorphic, nonzero section with

$$
H(s, s)=\left(\sum_{i=1}^{r} c_{i} \log \left|z_{i}\right|+c\right)^{2},
$$

for some $r \leq n$ and constants $c_{i}$ and $c$ with $c_{i}<0$. Then $H$ is a good metric on $L$. 
Proof Let $h=H(s, s)$. Clearly,

$$
h, h^{-1}<O\left(\sum_{i=1}^{r} \log \left|z_{i}\right|\right)^{2},
$$

so it remains to show that $\alpha=\partial h / h$ is a good 1 -form.

We have

$$
\alpha=\frac{\sum_{i} \frac{c_{i} d z_{i}}{z_{i}}}{\sum_{i} c_{i} \log \left|z_{i}\right|+c},
$$

so

$$
\begin{aligned}
\left|\alpha\left(\frac{\partial}{\partial z_{j}}\right)\right| & =\frac{c_{j}}{\left|z_{j}\right|\left|\sum_{i} c_{i} \log \right| z_{i}|+c|} \\
& \leq \frac{1}{\left|z_{j}\right| \log \left|z_{j}\right|} \\
& =\omega\left(\frac{\partial}{\partial z_{j}}, \frac{\partial}{\partial z_{j}}\right)^{1 / 2} .
\end{aligned}
$$

Thus $\alpha$ has Poincaré growth. We have

$$
\begin{aligned}
d \alpha & =\frac{1}{2} \frac{\sum_{i j} c_{i} c_{j} \frac{d z_{i} \wedge d \bar{z}_{j}}{z_{i} \bar{z}_{j}}}{\left(\sum c_{i} \log \left|z_{i}\right|+c\right)^{2}}, \\
\left|d \alpha\left(\frac{\partial}{\partial z_{k}}, \frac{\partial}{\partial \bar{z}_{l}}\right)\right| & =\frac{1}{2} \frac{c_{k} c_{l}}{\left|z_{k} z_{l}\right|\left(\sum_{i} c_{i} \log \left|z_{i}\right|+c\right)^{2}} \\
& \leq \frac{1}{4} \frac{1}{\left|z_{k}\right| \log \left|z_{k}\right|} \frac{1}{\left|z_{l}\right| \log \left|z_{l}\right|} \\
& =\frac{1}{4} \omega\left(\frac{\partial}{\partial z_{k}}, \frac{\partial}{\partial z_{k}}\right)^{1 / 2} \omega\left(\frac{\partial}{\partial \bar{z}_{l}}, \frac{\partial}{\partial \bar{z}_{l}}\right)^{1 / 2} .
\end{aligned}
$$

Thus, $d \alpha$ also has Poincaré growth, so $\alpha$ is a good 1 -form, and $H$ is a good metric.

Proof of Theorem 9.8 We need to give holomorphic sections of $Q Y_{D}$ around every point of $\bar{S}_{D}^{1}$ and $C_{P}$ and show that the norms of these sections satisfy the required bounds.

If $p \in \bar{S}_{d^{2}}^{1}$, then we saw in Section 7.3 and Section 7.5 that $\bar{X}_{d^{2}}$ is normal around $p$, so we can work in $\bar{X}_{d^{2}}$. We gave a section $s$ of $\mathcal{O}(-1)$ around $p$ whose norm is given by (7-3) or (7-7). The norm of $s^{2}$ as a section of $\mathcal{O}(-2)$ is the square of the norm of $s$, and $s^{2}$ can be regarded as a section of $Q Y_{D}$ around $p$. Thus $h_{Q}$ is good around each $p \in \bar{S}_{d^{2}}^{1}$ by Lemma 9.9. 
If $p \in C_{P}^{0}$, then we can consider $\pi(p)$ as a point in $\mathbb{P} \Omega \mathcal{D}_{2}^{0}\left(T_{2,0}\right)$ or $\mathbb{P} \Omega \mathcal{D}_{2}^{0}\left(T_{2,1}\right)$. As we saw in Section 7.6, $\pi(p)$ is contained in a hypersurface $U_{\lambda}$ with coordinates $(x, y, z)$ on $U_{\lambda}$. The variety $\bar{X}_{D}$ is contained in $U_{\lambda}$ near $\pi(p)$ and is cut out by the equation, (7-12). In a neighborhood of $p$ in $Y_{D}$, there are coordinates $(u, v)$ with

$$
x=u, y=v^{q}, \text { and } z=\theta v^{r},
$$

for some $p, q \in \mathbb{N}$ and root of unity $\theta$ by Proposition 8.1. We defined a section $s$ of $\mathcal{O}(-1)$ over $U_{\lambda}$ with norm given by (7-18). The pullback of $s^{2}$ to $Y_{D}$ gives a section $t$ of $Q Y_{D}$ with norm,

$$
h_{Q}(t, t)=\left(c_{1} \log |v|+c\right)^{2},
$$

for some constants $c_{1}$ and $c$ with $c_{1}<0$. Thus by Lemma 9.9, $h_{Q}$ is good around every $p \in C_{P}^{0}$.

Now suppose $p=c_{P}$ for some prototype $P$. We can consider $\pi(p)$ as a point in $\mathbb{P} \Omega \mathcal{D}_{2}^{0}\left(T_{3,0}\right)$ or $\mathbb{P} \Omega \mathcal{D}_{2}^{0}\left(T_{3,0}^{3}\right)$. As we saw in Section $7.7, \pi(p)$ is contained in a hypersurface $U_{\lambda}$ with are coordinates $(x, y, z)$ on $U_{\lambda}$. The variety $\bar{X}_{D}$ is contained in $U_{\lambda}$ near $\pi(p)$ and is cut out by the equation, (7-21). In a neighborhood of $p$ in $Y_{D}$, there are coordinates $(u, v)$ with

$$
x=u^{p} v^{q}, y=u^{r}, \text { and } z=v^{s},
$$

for some $p, q, r, s \in \mathbb{N}$ by Proposition 8.3. We defined a section $s$ of $\mathcal{O}(-1)$ over $U_{\lambda}$ with norm given by (7-23). The pullback of $s^{2}$ to $Y_{D}$ gives a section $t$ of $Q Y_{D}$ with norm,

$$
h_{Q}(t, t)=\left(c_{1} \log |u|+c_{2} \log |v|\right)^{2},
$$

for some negative constants $c_{1}$ and $c_{2}$. Thus by Lemma $9.9, h_{Q}$ is good around every $c_{P}$.

Corollary 9.10 The first Chern class of $Q^{i} Y_{D}$ is

$$
c_{1}\left(Q^{i} Y_{D}\right)=\left[\omega_{i}\right]
$$

Proof Since $h_{Q}$ is a good metric, $c_{1}\left(Q Y_{D}, h_{Q}\right)$ represents $c_{1}\left(Q Y_{D}\right)$ by Theorem 9.4. We showed in Proposition 2.8 that $c_{1}\left(Q Y_{D}, h_{Q}\right)=\omega_{1}$. This shows that $c_{1}\left(Q^{1} Y_{D}\right)=$ $\left[\omega_{1}\right]$. Also,

$$
c_{1}\left(Q^{2} Y_{D}\right)=c_{1}\left(\tau^{*} Q^{1} Y_{D}\right)=\tau^{*} c_{1}\left(Q^{1} Y_{D}\right)=\left[\omega_{2}\right] .
$$

Corollary 9.11 For $i=1,2$, and for any nondegenerate $Y_{D}$-prototype,

$$
\left[\omega_{i}\right] \cdot\left[C_{P}\right]=0 .
$$

Proof This pairing is equal to the degree of $Q Y_{D}$ restricted to $C_{P}$. Since this restriction is trivial by Proposition 9.7, the degree is zero. 


\subsection{Extension of the foliation $\mathcal{A}_{D}$ to $Y_{D}$}

Proposition 9.12 The foliation $\mathcal{A}_{D}$ of $X_{D}$ extends to a foliation (which we will continue to call $\mathcal{A}_{D}$ ) of

$$
Y_{D} \backslash \bigcup_{P} c_{P}
$$

where the union is over all nonterminal $Y_{D}$-prototypes. The curves $C_{P}^{0}$ and $S_{D}^{1}$ are leaves of $\mathcal{A}_{D}$, and $\mathcal{A}_{D}$ is transverse to $\bar{S}_{D}^{2}$.

Proof It follows directly from the equations for $\mathcal{A}_{D}$ given in Corollaries 7.7 and 7.12 that $\mathcal{A}_{D}$ extends over $S_{D}^{1}$ and $S_{D}^{2}$ to a foliation which contains $S_{D}^{1}$ as a leaf and is transverse to $S_{D}^{2}$. The equations for $\mathcal{A}_{D}$ in Corollary 7.15 show that $\mathcal{A}_{D}$ extends over the intersection points of $S_{D}^{1}$ and $S_{D}^{2}$ and is transverse to $S_{D}^{2}$ there. For $p \in C_{P}^{0}$, equations for $\mathcal{A}_{D}$ near $\pi(p) \in \bar{X}_{D}$ are given in Theorem 7.22. There are local coordinates $(u, v)$ in a neighborhood of $p$ in $Y_{D}$ such that $x=u, y=v^{p}$, and $z=\theta v^{q}$ for some $p, q \in \mathbb{N}$ and root of unity $q$. In these coordinates, $C_{P}$ is cut out by $v=0$, and $\mathcal{A}_{D}$ is given by $v=$ const. Thus $\mathcal{A}_{D}$ extends over $C_{P}^{0}$ to a foliation which contains $C_{P}^{0}$ as a leaf.

We will now study $T^{*} \mathcal{A}_{D}$, the cotangent bundle to the leaves of $\mathcal{A}_{D}$. Since every orbifold line bundle on a complex orbifold minus a subvariety of codimension at least two extends to an orbifold line bundle over the entire orbifold, we can regard $T^{*} \mathcal{A}_{D}$ as an orbifold bundle over all of $Y_{D}$, even though the foliation is singular at the points $c_{P}$. The holomorphic sections of $\mathcal{A}_{D}$ over an open set $U$ are exactly the holomorphic sections over $U$ minus the singular points of $\mathcal{A}_{D}$.

Proposition 9.13 The only singular fibers (that is, fibers which are nontrivial quotients $\mathbb{C} / G)$ of $T^{*} \mathcal{A}_{D}$ are over the elliptic points of $X_{D}$ and the elliptic points of $S_{d^{2}}^{i}$. For each nonterminal $Y_{D}$-prototype $P$, the restriction of $T^{*} \mathcal{A}_{D}$ to the curve $C_{P}$ is trivial. For each terminal $Y_{D}$-prototype, the restriction of $T^{*} \mathcal{A}_{D}$ to the curve $C_{P}$ is isomorphic to $T^{*} C_{P}\left(c_{P^{-}}\right)$.

Proof Besides the elliptic points, the only points over which $T^{*} \mathcal{A}_{D}$ could have singular fibers are the singular points $c_{P}$ of $\mathcal{A}_{D}$. Every section of an orbifold line bundle vanishes on a singular fiber, so to show that the fiber over $c_{P}$ is nonsingular, it suffices to show that $T^{*} \mathcal{A}_{D}$ has a nonzero section over $c_{P}$.

Let $(x, y, z)$ be the coordinates on in a neighborhood of $\pi\left(c_{P}\right)$ in the hypersurface $U_{\lambda} \subset$ $\mathbb{P} \Omega \mathcal{D}_{2}\left(T_{3,0}\right)$ as in Section 7.7. By Theorem 7.27 and Proposition 8.3, a neighborhood 
of $c_{P}$ in $Y_{D}$ is of the form $\Delta^{2} / G$, where $\Delta^{2}$ is a polydisk with coordinates $(u, v)$ such that

$$
x=u^{p} v^{q}, \quad y=u^{r}, \quad z=v^{s}
$$

for some $p, q, r, s \in \mathbb{N}$, and $G$ is a cyclic group whose action on $\Delta^{2}$ is generated by

$$
(u, v)=\left(\theta u, \theta^{n} v\right)
$$

for some root of unity $\theta$. By the equation,

$$
y^{\lambda} z^{\lambda-1}=\mathrm{const}
$$

for the foliation $\mathcal{A}_{D}$ from Theorem $7.27, \mathcal{A}_{D}$ is given on $\Delta^{2}$ by the equation,

$$
u^{a} v^{b}=\mathrm{const},
$$

for some $a, b \geq 0$. If $P$ is nonterminal, then $\lambda>1$, and $a, b>0$. Otherwise $\lambda=1$, and $b=0$, so the foliation is nonsingular at $c_{P}$. Define a vector field $X$ on $\Delta^{2}$ by

$$
X=b u \frac{\partial}{\partial u}-a v \frac{\partial}{\partial v} \text {. }
$$

$X$ is tangent to $\mathcal{A}_{D}$ and is invariant under $G$, so it gives a nonzero section of $T^{*} \mathcal{A}_{D}$ in a neighborhood of $c_{P}$. Thus the fiber of $T^{*} \mathcal{A}_{D}$ over $c_{P}$ is not singular.

The restriction of $X$ to $C_{P}$ is a vector field on $C_{P}$ in a neighborhood of $c_{P}$ which vanishes at $c_{P}$. That means that a vector field $Y$ on $C_{P}$ defines a nonzero section of $\left.T^{*} \mathcal{A}_{D}\right|_{C_{P}}$ if and only if $Y$ vanishes at $c_{P}$. If $P$ is nonterminal, then $C_{P}$ has two points $c_{P}$ and $c_{P^{-}}$which pass through singular points of $\mathcal{A}_{D}$. A holomorphic vector field on $C_{P}$ which has a zero at each of these points and no other zeros determines a nonzero holomorphic section of $\left.T^{*} \mathcal{A}_{D}\right|_{C_{P}}$. Thus this restriction is trivial.

If $P$ is terminal, then $c_{P}$ is a nonsingular point of $\mathcal{A}_{D}$. Thus, the only singular point of $\mathcal{A}_{D}$ which $C_{P}$ intersects is $c_{P^{-}}$, so

$$
\left.T^{*} \mathcal{A}_{D}\right|_{C_{P}} \cong T^{*} C_{P}\left(c_{P^{-}}\right) \text {. }
$$

Corollary 9.14 The pairings of $c_{1}\left(T^{*} \mathcal{A}_{D}\right)$ with the fundamental classes $\left[C_{P}\right]$ are

$$
c_{1}\left(T^{*} \mathcal{A}_{D}\right) \cdot\left[C_{P}\right]= \begin{cases}0 & \text { if } P \text { is not terminal } \\ -1 & \text { if } P \text { is terminal. }\end{cases}
$$

Proof The pairing $c_{1}\left(T^{*} \mathcal{A}_{D}\right) \cdot\left[C_{P}\right]$ is equal to the degree of the restriction of $T^{*} \mathcal{A}_{D}$ to $C_{P}$. If $P$ is nonterminal, then this restriction is trivial, so the pairing is zero. If $P$ is terminal, then this restriction has degree -1 by Proposition 9.13. 
Chern class of $T^{*} \mathcal{A}_{D}$ when $\mathrm{D}$ is not square We now calculate the first Chern class of $T^{*} \mathcal{A}_{D}$ by relating it to the bundle $Q^{2} Y_{D}$.

Lemma 9.15 If $D$ is not square, and $\mathcal{L} \rightarrow Y_{D}$ is an orbifold line bundle with a nonzero holomorphic section $s \in \Gamma\left(X_{D}, \mathcal{L}\right)$ defined over $X_{D}$, then $s$ is a meromorphic section of $\mathcal{L}$ over $Y_{D}$.

Proof Let $\mathcal{O}(\mathcal{L})$ be the sheaf of sections of $\mathcal{L}$. By [71], $\mathcal{O}(\mathcal{L})$ is a coherent algebraic sheaf on $Y_{D}$. We claim that $s$ is defined algebraically over $X$. The lemma would follow from this because every algebraic section of a line bundle over a dense, Zariski-open subset is meromorphic by [33, Lemma II.5.3].

The direct image $\pi_{*} \mathcal{O}(\mathcal{L})$ is a coherent algebraic sheaf on $\hat{X}_{D}$. Given an open set $U \subset \widehat{X}_{D}$ and $t \in \Gamma\left(U, \pi_{*} \mathcal{O}(\mathcal{L})\right)$, let $r=t / s$, a holomorphic function on $U \backslash C$, where $C=\widehat{X}_{D} \backslash X_{D}$ is the set of cusps of $X_{D}$. By Koecher's principle (see van der Geer [75]), $r$ extends uniquely to a holomorphic function $\widetilde{r}$ on $U$. This defines an injective analytic map of sheaves,

$$
i: \pi_{*} \mathcal{O}(\mathcal{L}) \rightarrow \mathcal{O}_{\hat{X}_{D}}
$$

with $i(t)=\tilde{r}$. By [71], $i$ is actually defined algebraically. Since $i(s)=1$, and 1 is algebraic, it follows that $s$ is an algebraic section of $\mathcal{L}$ over $X_{D}$.

Theorem 9.16 When $D$ is not square,

$$
Q^{2} Y_{D} \cong T^{*} \mathcal{A}_{D}
$$

Proof Define

$$
\mathcal{L}=Q^{2} Y_{D} \otimes\left(T^{*} \mathcal{A}_{D}\right)^{-1} .
$$

By Proposition 2.8 and Proposition 2.9,

$$
\left.\left.Q^{2} Y_{D}\right|_{X_{D}} \cong L_{2} \cong T^{*} \mathcal{A}_{D}\right|_{X_{D}},
$$

so there is a nonzero, holomorphic section $s$ of $\mathcal{L}$ over $X_{D}$, which is a meromorphic section of $\mathcal{L}$ over $Y_{D}$ by Lemma 9.15.

The divisor of $s$ is

$$
(s)=\sum_{P} e_{P} C_{P}
$$

for some integers $e_{P}$, so

$$
c_{1}(\mathcal{L})=\sum_{P} e_{P}\left[C_{P}\right]
$$


We need to show that the $e_{P}$ are all zero, for then $s$ would be nonzero and holomorphic, so $\mathcal{L}$ would be trivial. By Proposition 9.7 and Proposition 9.13, the restriction of $\mathcal{L}$ to each $C_{P}$ is trivial, so

$$
\left[C_{Q}\right] \cdot \sum e_{P}\left[C_{P}\right]=\left[C_{Q}\right] \cdot c_{1}(\mathcal{L})=0
$$

for each $Y_{D}$-prototype $Q$. It then follows from Theorem 8.11 that $e_{P}=0$ for each $P$.

Corollary 9.17 If $D$ is not square, then the first Chern class of $T^{*} \mathcal{A}_{D}$ is

$$
c_{1}\left(T^{*} \mathcal{A}_{D}\right)=\left[\omega_{2}\right] .
$$

Chern class of $\boldsymbol{T}^{*} \mathcal{A}_{\boldsymbol{d}^{2}}$ The foliation $\mathcal{A}_{d^{2}}$ of $X_{d^{2}}$ extends to a foliation $\widehat{\mathcal{A}}_{d^{2}}$ of the orbifold $\widehat{X}_{d^{2}}$. Give $T \widehat{\mathcal{A}}_{d^{2}}$ the metric $\rho$ induced by the hyperbolic metric along the leaves of $\mathcal{A}_{D}$. This metric is singular along $\bar{R}_{d^{2}}^{2}$.

Theorem 9.18 The metric $\rho$ is a good metric for $T \widehat{\mathcal{A}}_{d^{2}}\left(-\bar{R}_{d^{2}}^{2}\right)$.

Proof Let $G \subset \mathrm{SL}_{2} \mathcal{O}_{d^{2}}$ be the cyclic subgroup generated by

$$
\left(\left(\begin{array}{ll}
1 & 0 \\
0 & 1
\end{array}\right),\left(\begin{array}{ll}
1 & d \\
0 & 1
\end{array}\right)\right)
$$

We then have a map,

$$
\mathbb{W} \times \mathbb{W} / G \cong \mathbb{W} \times \Delta^{*} \rightarrow X_{d^{2}}
$$

(where $\Delta^{*}$ is the punctured unit disk), which extends to an unramified map of orbifolds,

$$
p: \mathbb{H} \times \Delta \rightarrow \widehat{X}_{d^{2}},
$$

sending $\mathbb{W} \times\{0\}$ to $R_{d^{2}}^{2}$, and sending leaves of the vertical foliation by disks to leaves of $\widehat{\mathcal{A}}_{d^{2}}$. The vector field,

$$
X=z_{2} \frac{\partial}{\partial z_{2}}
$$

on $\mathbb{H} \times \Delta^{*}$ locally defines a nonzero, holomorphic section of $T \widehat{\mathcal{A}}_{d^{2}}\left(-\bar{R}_{d^{2}}^{2}\right)$ around points in $R_{d^{2}}^{2}$. The norm of this section is

$$
\rho(X, X)=\left(\log \left|z_{2}\right|\right)^{-2},
$$

so by Lemma 9.9, $\rho$ is good near points of $R_{d^{2}}^{2}$ (note that the exponent of the metric in Lemma 9.9 is irrelevant because changing the exponent only changes $\partial h / h$ by a constant).

The proof that $\rho$ is good near the cusps of $\hat{X}_{D}$ is the same, except these points are covered by $\Delta \times \Delta$, rather then $\mathbb{W} \times \Delta$. 
Corollary 9.19 The first Chern class of $T^{*} \mathcal{A}_{d^{2}}$ is

$$
c_{1}\left(T^{*} \mathcal{A}_{d^{2}}\right)=\left[\omega_{2}\right]-\left[\bar{S}_{d^{2}}^{2}\right] .
$$

Proof By Theorem 9.18,

$$
c_{1}\left(T^{*} \widehat{\mathcal{A}}_{d^{2}}\right)=\left[\omega_{2}\right]-\left[\bar{R}_{d^{2}}^{2}\right] .
$$

Since $\pi^{*}\left(T^{*} \widehat{\mathcal{A}}_{d^{2}}\right)$ is isomorphic to $T^{*} \mathcal{A}_{d^{2}}$ over $X_{d^{2}}$,

$$
\pi^{*}\left(T^{*} \widehat{\mathcal{A}}_{d^{2}}\right)=T^{*} \mathcal{A}_{d^{2}}\left(\sum_{P} e_{P} C_{P}\right)
$$

for some integers $e_{P}$, where the sum is over all nondegenerate $Y_{D}$-prototypes $P$. Thus

$$
\sum_{P} e_{P}\left[C_{P}\right]=\left[\omega_{2}\right]-\left[\bar{S}_{d^{2}}^{2}\right]-c_{1}\left(T^{*} \mathcal{A}_{d^{2}}\right)
$$

because $\pi^{*}\left[\bar{R}_{d^{2}}^{2}\right]=\left[\bar{S}_{d^{2}}^{2}\right]$.

The pairing of $\left[C_{P}\right]$ with the right hand side of (9-12) is trivial for all $P$ by Corollary 9.14 together with the fact that from Theorem 8.7 that $C_{P}$ is disjoint from $\bar{S}_{d^{2}}^{2}$ if $P$ is nonterminal, and $C_{P}$ intersects $\bar{S}_{d^{2}}^{2}$ in one transverse intersection if $P$ is terminal. Theorem 8.11 then implies that the $e_{P}$ are all zero. Thus $c_{1}\left(T^{*} \mathcal{A}_{d^{2}}\right)$ is as claimed.

\section{Euler characteristic of $W_{D}$}

In this section, we calculate $\chi\left(W_{D}\right)$. We construct a meromorphic section of a line bundle over $Y_{D}$ which vanishes along $\bar{W}_{D}$ and has simple poles along $\bar{P}_{D}$ and $\bar{S}_{D}^{2}$. This allows us to relate $\chi\left(W_{D}\right)$ to $\chi\left(P_{D}\right), \chi\left(X_{D}\right)$, and $\chi\left(S_{D}^{2}\right)$.

In [58], McMullen defined a quadratic differential on the leaves of $\mathcal{A}_{D}$ in $X_{D}$, and used this to construct a transverse measure for the foliation of $X_{D}$ by $\mathrm{SL}_{2} \mathbb{R}$ orbits. This quadratic differential was also studied in [70] in the case when $D$ is square. Here we recall the construction of this quadratic differential from [58] and define it on all of $Y_{D}$.

Theorem 10.1 On each leaf $L$ of $\mathcal{A}_{D}$, there is locally a quadratic differential $q$ which has a simple zero on each point of $\bar{W}_{D} \cap L$, has a simple pole on each point of $\left(\bar{P}_{D} \cup \bar{S}_{D}^{2}\right) \cap L$, and is elsewhere holomorphic and nonzero. 
Proof For each $z \in L$, let $X_{z}$ be the corresponding Riemann surface with real multiplication. Choose a basepoint $z_{0} \in L$, and choose some eigenform $\omega_{z_{0}} \in \Omega^{1}\left(X_{z_{0}}\right)$. There is a unique section $z \mapsto \omega_{z}$ of $\Omega Y_{D}$ over $L$ such that the absolute periods of the $\omega_{z}$ are locally constant. When $L \subset X_{D}$, this follows from Proposition 2.10. If $L=C_{P}$, this is clear because the periods of $\omega_{z}$ are determined by the residues at the nonseparating nodes of $X_{z}$, and along $C_{P}$ these residues have constant ratio, and if $L=S_{d^{2}}^{1}$, this is also clear because if $\left(X_{z}, \omega_{z}\right)$ is a cylinder covering differential, then the periods of $\omega_{z}$ are all rational multiples of the period around the node of $X_{z}$, so if this period is constant along $L$, then all of the periods are constant.

If $z \in L \backslash\left(\bar{W}_{D} \cup \bar{P}_{D} \cup \bar{S}_{D}^{2}\right)$, then $\left(X_{z}, \omega_{z}\right)$ has two distinct simple zeros. Let

$$
f(z)=\int_{\gamma} \omega_{z}
$$

where $\gamma$ is some path joining the zeros of $\omega_{z}$. This $f(z)$ is a multivalued holomorphic function on $L \backslash\left(\bar{W}_{D} \cup \bar{P}_{D} \cup \bar{S}_{D}^{2}\right)$ because the value of $f(z)$ depends on a choice of an oriented path joining the zeros of $\omega_{z}$. Define

$$
q=(\partial f)^{2} .
$$

We claim that $q$ is a well-defined quadratic differential on $L$. To see this, suppose we replace an oriented path $\gamma$ joining the zeros $p$ and $q$ of $\omega_{z}$ with a new path $\gamma^{\prime}$ joining $p$ to $q$. Since $\gamma-\gamma^{\prime}$ is a closed path, this changes $f$ by an absolute period of $\omega_{z}$. Since the absolute periods of $\omega_{z}$ are constant along $L$, replacing $\gamma$ with $\gamma^{\prime}$ does not change $\partial f$. If we replace $\gamma$ with $-\gamma$, the same path with the opposite orientation, this changes $f$ to $-f$, which does not affect $q$ because of the exponent. Thus $q$ is well defined.

We now identify the zeros and poles of $q$. Since the absolute and relative periods give a system of local coordinates on the strata in $\Omega \overline{\mathcal{M}}_{2}$, the relative periods give local coordinates on $L$ because the absolute periods are constant, so $q$ is holomorphic and nonzero on the complement of $\bar{P}_{D}, \bar{W}_{D}$, and $\bar{S}_{D}^{2}$.

Suppose $z \in L \cap \bar{W}_{D}$. For $z \in \mathbb{C}$, let $I(z) \in \mathbb{C}$ be the segment joining 0 to $z$. For $w \in \Delta_{\epsilon}$, where $\Delta_{\epsilon}$ is some small $\epsilon$-ball around 0 in $\mathbb{C}$, let

$$
\phi(w)=\left(X_{w}, \omega_{w}\right) \#_{I\left(w^{3 / 2}\right)},
$$

(this is the operation of splitting a double zero defined in Section 4.1). As in Section 6.2, $\phi$ is a conformal isomorphism onto some neighborhood of $z$ in $\mathbb{C}$. In these coordinates, we have $f(w)=w^{3 / 2}$, so

$$
q=\frac{9}{4} w d w^{2} .
$$


Thus $q$ has a simple zero at $z$.

Now suppose $z \in L \cap \bar{P}_{D}$. Then

$$
\left(X_{z}, \omega_{z}\right)=\left(X_{1}, \omega_{1}\right) \#\left(X_{2}, \omega_{2}\right),
$$

the one-point connected sum of two genus one differentials. Define a conformal mapping $\phi: \Delta_{\epsilon} \rightarrow L$ by

$$
\phi(w)=\left(X_{1}, \omega_{1}\right) \#_{I\left(w^{1 / 2}\right)}\left(X_{2}, \omega_{2}\right),
$$

taking a connected sum along $I\left(w^{1 / 2}\right)$. Similarly, if $z \in L \cap \bar{S}_{D}^{2}$, then we can regard $\left(X_{z}, \omega_{z}\right)$ as an genus one differential with two points identified to a node. Define a conformal mapping $\phi: \Delta_{\epsilon} \rightarrow L$ by

$$
\phi(w)=\left(X_{z}, \omega_{z}\right) \#_{I\left(w^{1 / 2}\right)},
$$

taking a self-connected sum along $I\left(w^{1 / 2}\right)$ as described in Section 4.1. In either case, we have in these coordinates $f(w)=w^{1 / 2}$, so

$$
q=\frac{1}{2} w^{-1} d w^{2}
$$

Thus $q$ has a simple pole at $z$.

This construction locally defines meromorphic sections of $\left(T^{*} \mathcal{A}_{D}\right)^{2}$, but does not give a global section. The problem is that the definition of the quadratic differential $q$ on a leaf $L$ depended on a choice of $\omega_{z_{0}} \in \Omega\left(X_{z_{0}}\right)$ for some basepoint $z_{0} \in L$. There is no obvious way to choose these quadratic differentials coherently to get a global section. To get a global section of a bundle, we must twist by some power of $\Omega Y_{D}$.

Theorem 10.2 There is a meromorphic section of the line bundle

$$
\mathcal{L}=\left(Q Y_{D}\right)^{*} \otimes\left(T^{*} \mathcal{A}_{D}\right)^{2}
$$

on $Y_{D}$ which has a simple zero along $\bar{W}_{D}$, has a simple pole along $\bar{P}_{D}$ and $\bar{S}_{D}^{2}$ (which is empty if $D$ is nonsquare), and is elsewhere nonzero and finite.

Proof The construction in the proof of Theorem 10.1 defined for each point,

$$
z \in Y_{D} \backslash \bigcup c_{P}
$$

together with a choice of $\omega \in \Omega^{1}\left(X_{z}\right)$ a quadratic differential $q$ on the leaf of $\mathcal{A}_{D}$ through $z$. Thus, we have a map $h:\left.\Omega^{1}\left(X_{z}\right) \rightarrow\left(T^{*} \mathcal{A}_{D}\right)^{2}\right|_{z}$. With $f$ as in (10-1), if we replace $\omega \in \Omega^{1}\left(X_{z}\right)$ with $a \omega$, then $f$ becomes $a f$, and $q$ becomes $a^{2} q$. Thus $h$ satisfies

$$
h(a \omega)=a^{2} h(\omega) .
$$


We can thus regard $h$ as a linear map

$$
\left.\Omega^{1}\left(X_{z}\right)^{\otimes 2} \rightarrow\left(T^{*} \mathcal{A}_{D}\right)\right|_{z}
$$

This defines a meromorphic section $s$ of $\mathcal{L}$ over $Y_{D} \backslash \bigcup c_{P}$ which has the same zeros and poles as the quadratic differentials on the leaves of $\mathcal{A}_{D}$. This section extends holomorphically over the points $c_{P}$ to give a section over all of $Y_{D}$ because any holomorphic section of a line bundle defined on a neighborhood of a normal point extends over that point.

Corollary 10.3 If $D$ is not square, then the fundamental class $\left[\bar{W}_{D}\right]$ of $\bar{W}_{D}$ in $H^{2}\left(Y_{D} ; \mathbb{Q}\right)$ is given by

$$
\left[\bar{W}_{D}\right]=\left[\bar{P}_{D}\right]-\left[\omega_{1}\right]+2\left[\omega_{2}\right] .
$$

The fundamental class $\left[\bar{W}_{d^{2}}\right]$ of $\bar{W}_{d^{2}}$ in $H^{2}\left(Y_{d^{2}} ; \mathbb{Q}\right)$ is given by

$$
\left[\bar{W}_{d^{2}}\right]=\left[\bar{P}_{d^{2}}\right]-\left[\bar{S}_{d^{2}}^{2}\right]-\left[\omega_{1}\right]+2\left[\omega_{2}\right] .
$$

Proof By [27, p 141], for any line bundle $L$ over a compact, complex manifold $M$ with a meromorphic section $s$ of $L$ having divisor $D$,

$$
c_{1}(L)=[D] .
$$

This is proved for complex manifolds, but this is still true and the proof works just as well for orbifolds.

In our situation, the section $s$ of $\mathcal{L}$ from Theorem 10.2 implies

$$
\left[\bar{W}_{D}\right]-\left[\bar{P}_{D}\right]-\left[\bar{S}_{D}^{2}\right]=c_{1}(\mathcal{L})=-c_{1}\left(Q Y_{D}\right)+2 c_{1}\left(T^{*} \mathcal{A}_{D}\right) .
$$

This, together with (9-9), (9-10), and (9-11) yield the desired formulas.

Corollary 10.4 If $D \neq 1$ is a fundamental discriminant, then

$$
\begin{aligned}
\chi\left(W_{f^{2} D}\right) & =\chi\left(P_{f^{2} D}\right)-2 \chi\left(X_{f^{2} D}\right) \\
& =-9 \zeta_{K_{D}}(-1) f^{3} \sum_{r \mid f}\left(\frac{D}{r}\right) \frac{\mu(r)}{r^{2}} .
\end{aligned}
$$

If $D=d^{2}$, then

$$
\begin{aligned}
\chi\left(W_{d^{2}}\right) & =\chi\left(P_{d^{2}}\right)-\chi\left(S_{d^{2}}^{2}\right)-2 \chi\left(X_{d^{2}}\right) \\
& =-\frac{1}{16} d^{2}(d-2) \sum_{r \mid d} \frac{\mu(r)}{r^{2}} .
\end{aligned}
$$


Proof If we pair $-\left[\omega_{1}\right]$ with both sides of (10-2) and (10-3), then by Proposition 9.5 and Proposition 9.6, we get

$$
\begin{aligned}
& -\int_{W_{D}} \omega_{1}=-\int_{P_{D}} \omega_{1}-2 \int_{X_{D}} \omega_{1} \wedge \omega_{2} \\
& -\int_{W_{d^{2}}} \omega_{1}=-\int_{P_{d^{2}}} \omega_{1}-2 \int_{X_{d^{2}}} \omega_{1} \wedge \omega_{2}+\int_{S_{d^{2}}^{2}} \omega_{1} .
\end{aligned}
$$

Since $W_{D}, P_{D}$, and $S_{d^{2}}^{2}$ are transverse to $\mathcal{A}_{D}$, the form $-\omega_{1}$ restricts to the Chern form of the hyperbolic metric on these Riemann surfaces. Thus by the Gauss-Bonnet theorem we get (10-4) and (10-6). We calculated $\chi\left(X_{D}\right)$ and $\chi\left(P_{D}\right)$ in Theorem 2.12 and Theorem 2.22. We will calculate $\chi\left(S_{d^{2}}^{2}\right)$ in the following proposition. Putting all of this together yields (10-5) and (10-7).

Proposition 10.5 When $d>2$, we have

$$
\begin{aligned}
\chi\left(S_{4}^{i}\right) & =-\frac{1}{2} \\
\chi\left(S_{d^{2}}^{i}\right) & =-\frac{1}{12} d^{2} \sum_{r \mid d} \frac{\mu(r)}{r^{2}} .
\end{aligned}
$$

Proof We know that

$$
S_{d^{2}}^{i} \cong \mathbb{H} / \Gamma_{1}(d) .
$$

It follows from [59, Theorem 4.2.5] that

$$
\chi\left(\mathbb{M} / \Gamma_{1}(d)\right)= \begin{cases}-\frac{1}{2} & \text { if } d=2 ; \\ -\frac{1}{12} \phi(d) d \prod_{p \mid d}\left(1+\frac{1}{p}\right) & \text { when } d>2,\end{cases}
$$

where $\phi$ is the Euler $\phi$-function, and the product is over all primes dividing $d$. By [36, Proposition 2.2.5],

$$
\phi(d)=d \prod_{p \mid d}\left(1-\frac{1}{p}\right)
$$

so when $d>2$,

$$
\chi\left(\mathbb{H} / \Gamma_{1}(d)\right)=-\frac{1}{12} d^{2} \prod_{p \mid d}\left(1-\frac{1}{p^{2}}\right)=-\frac{0}{12} d^{2} \sum_{r \mid d} \frac{\mu(r)}{r^{2}},
$$

as claimed. 


\section{Fundamental class of $\bar{W}_{D}$}

In this section, we will calculate the fundamental class of $\bar{W}_{D}$. By Corollary 10.3, we just need to know the fundamental classes of $\bar{S}_{D}^{i}$ and $\bar{P}_{D}$. Recall that we defined $\pi_{B}$ to be the orthogonal projection of $H^{2}\left(Y_{D} ; \mathbb{Q}\right)$ onto the subspace $B$ generated by the curves $C_{P}$.

Theorem 11.1 The fundamental class of $\bar{S}_{d^{2}}^{i}$ in $H^{2}\left(Y_{d^{2}} ; \mathbb{Q}\right)$ is

$$
\left[\bar{S}_{d^{2}}^{i}\right]=\frac{6}{d}\left[\omega_{i}\right]+\pi_{B}\left[\bar{S}_{d^{2}}^{i}\right] .
$$

Proof From Theorem 8.10, we have a map $p: Y_{d^{2}} \rightarrow \widehat{X}_{d^{2}}$ which collapses the curves $C_{P}$ to cusps of $\hat{X}_{d^{2}}$. Let

$$
q: \widehat{X}_{d^{2}} \rightarrow \overline{\mathbb{H} / \mathrm{SL}_{2} \mathbb{Z}}=\overline{\mathcal{M}}_{1,1}
$$

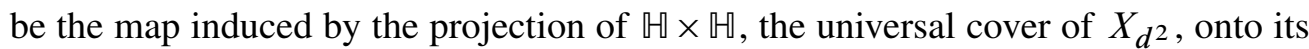
second factor. Let $r=q \circ p$, and let $\infty \in \overline{\mathcal{M}}_{1,1}$ by the single point added to $\mathbb{Z} / \mathrm{SL}_{2} \mathbb{Z}$. Then

$$
r^{-1}(\infty)=S_{d^{2}}^{1} \cup \bigcup_{P} C_{P}
$$

Let $f$ be a holomorphic function defined on a neighborhood of $\infty$ in $\overline{\mathcal{M}}_{1,1}$ which has a simple zero at $\infty$. We claim that $f \circ r$ vanishes to order $d$ along $S_{d^{2}}^{1}$.

It is enough to show that $f \circ q$ vanishes to order $d$ along $R_{d^{2}}^{1}$. Let $G \subset \mathrm{SL}_{2} \mathcal{O}_{d^{2}}$ be the cyclic group generated by

$$
\left(\left(\begin{array}{ll}
1 & 0 \\
0 & 1
\end{array}\right),\left(\begin{array}{ll}
1 & d \\
0 & 1
\end{array}\right)\right)
$$

and let $N \subset \mathrm{SL}_{2} \mathbb{Z}$ be the subgroup of upper-triangular matrices. We have the following commutative diagram:

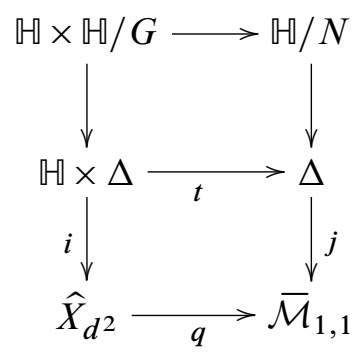

where $\Delta \subset \mathbb{C}$ is the unit disk. The two topmost vertical maps are isomorphisms onto $\mathbb{U} \times \Delta^{*}$ and $\Delta^{*}$ respectively, where $\Delta^{*}$ is the punctured disk. The maps $i$ and $j$ are 
unramified maps of orbifolds satisfying $i^{-1}\left(R_{d^{2}}^{1}\right)=\mathbb{H} \times\{0\}$ and $j^{-1}(\infty)=\{0\}$. The map $t$ is given by $t(z, w)=w^{d}$, and it follows that $f \circ q$ vanishes to order $d$ along $R_{d^{2}}^{1}$ as claimed because $f \circ j \circ t$ vanishes to order $d$ along $\mathbb{W} \times\{0\}$.

It follows from this claim that

$$
r^{*}[\infty]=d\left[\bar{S}_{d^{2}}^{1}\right]+\sum_{P} e_{P}\left[C_{P}\right],
$$

where $e_{P}$ is the order of vanishing of $f \circ r$ along $C_{P}$. Let $\eta$ be the 2 -form on $\mathbb{H} / \mathrm{SL}_{2} \mathbb{Z}$ induced by

$$
\frac{1}{2 \pi} \frac{d x \wedge d y}{y^{2}}
$$

on $\mathbb{H}$. Since $\chi\left(\mathbb{M} / \mathrm{SL}_{2} \mathbb{Z}\right)=-1 / 6$,

$$
\int_{\overline{\mathcal{M}}_{1,1}} \eta=\frac{1}{6}
$$

by the Gauss-Bonnet Theorem, so the cohomology class defined by the closed current $\langle\eta\rangle$ on $\overline{\mathcal{M}}_{1,1}$ satisfies

in $H^{2}\left(\overline{\mathcal{M}}_{1,1} ; \mathbb{Q}\right)$. We have

$$
6[\eta]=[\infty]
$$

so

$$
\begin{gathered}
r^{*} \eta=\omega_{1}, \\
r^{*}[\infty]=6\left[\omega_{1}\right] .
\end{gathered}
$$

This equation and Equation (11-2) imply (11-1) for $i=1$. The case $i=2$ follows from the same argument or by applying $\tau^{*}$ to (11-1).

Theorem 11.2 If $D$ is not square, then the fundamental class of $\bar{P}_{D}$ in $H^{2}\left(Y_{D} ; \mathbb{Q}\right)$ is

$$
\left[\bar{P}_{D}\right]=\frac{5}{2}\left(\left[\omega_{1}\right]+\left[\omega_{2}\right]\right)+\pi_{B}\left[\bar{P}_{D}\right] .
$$

The fundamental class of $\bar{P}_{d^{2}}$ in $H^{2}\left(Y_{d^{2}} ; \mathbb{Q}\right)$ is

$$
\left[\bar{P}_{d^{2}}\right]=\left(\frac{5}{2}-\frac{3}{d}\right)\left(\left[\omega_{1}\right]+\left[\omega_{2}\right]\right)+\pi_{B}\left[\bar{P}_{d^{2}}\right]
$$

Proof In $\overline{\mathcal{M}}_{2}$, let $\Delta_{0}$ be the divisor which is the closure of the locus of stable Riemann surfaces with one nonseparating node, and let $\Delta_{1}$ be the divisor which is the closure of the locus of stable Riemann surfaces with one separating node. Let $\delta_{i}=\left[\Delta_{i}\right]$, the fundamental class of $\Delta_{i}$. Define

$$
\lambda_{1}=c_{1}\left(\Omega \overline{\mathcal{M}}_{2}\right)
$$


These cohomology classes satisfy the well-known relation,

$$
\delta_{1}=5 \lambda_{1}-\frac{1}{2} \delta_{0},
$$

proved in [64].

Since $\pi_{2}^{*}\left(\Omega \overline{\mathcal{M}}_{2}\right)=\Omega^{1} Y_{D} \oplus \Omega^{2} Y_{D}$, where $\pi_{2}: Y_{D} \rightarrow \overline{\mathcal{M}}_{2}$ is the natural map, by Corollary 9.10 , we have

$$
\pi_{2}^{*}\left(\lambda_{1}\right)=\frac{1}{2}\left(\left[\omega_{1}\right]+\left[\omega_{2}\right]\right) .
$$

We claim that

$$
\pi_{2}^{*}\left(\delta_{1}\right)=\left[\bar{P}_{D}\right]
$$

Since $\pi_{2}^{-1}\left(\Delta_{1}\right)=\bar{P}_{D}$, it suffices to show that $\Delta_{1}$ is generically transverse to $\pi_{2}\left(Y_{D}\right)$. In $\mathcal{A}_{2}$, the divisor $\Delta_{1}$ corresponds to the surface $X_{1}$ parameterizing products of elliptic curves. In $\mathcal{A}_{2}$, the intersection of $X_{1}$ with $X_{D}$ is transverse because these are both linear subspaces of $\mathcal{A}_{2}$, so if their intersection was not transverse, one would be contained in the other. Equation (11-7) follows.

We now claim that

$$
\pi_{2}^{*}\left(\delta_{0}\right)=\left[\bar{S}_{D}^{1}\right]+\left[\bar{S}_{D}^{2}\right]+\sum_{P} e_{P}\left[C_{P}\right]
$$

for some integers $e_{P}$. Since

$$
\pi_{2}^{-1}\left(\Delta_{0}\right)=\bigcup_{i=1}^{2} \bar{S}_{D}^{i} \cup \bigcup_{P} C_{P},
$$

$\pi_{2} \circ \tau=\pi_{2}$, and $\tau^{*} S_{D}^{1}=S_{D}^{2}$, it suffices to show that $\Delta_{0}$ meets $\pi_{2}\left(Y_{D}\right)$ transversely along $\pi_{2}\left(S_{D}^{1}\right)$.

Let $p \in S_{d^{2}}^{1}$, and let $(X, \omega) \in \Omega \overline{\mathcal{M}}_{2}$ be a corresponding eigenform. We claim that $\pi_{2}\left(Y_{D}\right)$ meets $\Delta_{0}$ transversely at $\pi_{2}(p)$. Let $\left\{\alpha_{i}, \beta_{i}\right\}$ be a symplectic basis for $H_{1}(X ; \mathbb{Z})$ as in Section 6.3. Then we get coordinates $(v, w, x, y, z)$ on a neighborhood $U$ of $(X, \omega)$ in $\Omega \overline{\mathcal{M}}_{2}$ as in Section 6.3. The subspace $H$ of $U$ defined by the equations,

$$
v=\omega\left(\alpha_{1}\right), \quad w=\omega\left(\alpha_{2}\right),
$$

maps locally biholomorphically to $\overline{\mathcal{M}}_{2}$, so the coordinates $(x, y, z)$ on $H$ induce coordinates on $\overline{\mathcal{M}}_{2}$ on a neighborhood $W$ of $X$. In these coordinates,

$$
\Delta_{0}=V(z)
$$


By Corollary 7.7,

$$
\pi_{2}\left(Y_{D}\right) \cap W=V\left(x-\omega\left(\beta_{2}\right)\right) .
$$

Thus $\pi_{2}\left(Y_{D}\right)$ and $\Delta_{0}$ meet transversely at $p$ as claimed, and (11-8) follows.

Equations (11-3) and (11-4) then follow from (11-5), (11-6), (11-7), and (11-8).

Remark I am grateful to Gerard van der Geer for providing the idea of the proof of this theorem.

Remark We can also use (11-3) and (11-4) to get a new proof of Theorem 2.22. Since this proof doesn't use Siegel's formula, Theorem 2.16, this together with the previous proof of Theorem 2.22 can be used to give a proof of Siegel's formula.

Corollary 11.3 If $D$ is not square, then the fundamental class of $\bar{W}_{D}$ in $H^{2}\left(Y_{D} ; \mathbb{Q}\right)$ is given by

$$
\left[\bar{W}_{D}\right]=\frac{3}{2}\left[\omega_{1}\right]+\frac{9}{2}\left[\omega_{2}\right]+\pi_{B}\left[\bar{W}_{D}\right] .
$$

The fundamental class of $\bar{W}_{d^{2}}$ in $H^{2}\left(Y_{d^{2}} ; \mathbb{Q}\right)$ is given by

$$
\left[\bar{W}_{d^{2}}\right]=\frac{3}{2}\left(1-\frac{2}{d}\right)\left[\omega_{1}\right]+\frac{9}{2}\left(1-\frac{2}{d}\right)\left[\omega_{2}\right]+\pi_{B}\left[\bar{W}_{d^{2}}\right] .
$$

Proof This follows from plugging the formulas from Theorem 11.1 and Theorem 11.2 into the formulas from Corollary 10.3.

\section{Normal bundles}

We now study the normal bundles of the curves $\bar{W}_{D}, \bar{P}_{D}$, and $\bar{S}_{D}^{2}$ with the goal of calculating the self-intersection numbers of these curves. For any curve $C \subset Y_{D}$, we will write $N(C)$ for its normal bundle.

Proposition 12.1 For any connected component $C$ of $\bar{W}_{D}, \bar{P}_{D}$, or $\bar{S}_{D}^{2}$,

$$
\left.N(C) \cong T \mathcal{A}_{D}\right|_{C}
$$

as holomorphic line bundles over $C$.

Proof We claim that the foliation $\mathcal{A}_{D}$ is transverse to $\bar{W}_{D}, \bar{P}_{D}$, and $\bar{S}_{D}^{2}$. Equation (12-1) follows directly from this claim.

The curves $W_{D}$ and $P_{D}$ are transverse to $\mathcal{A}_{D}$ because the inverse images of these curves in $\mathbb{U} \times \mathbb{U}$ are the unions of graphs of holomorphic functions $\mathbb{H} \rightarrow \mathbb{H}$. The curve 
$S_{D}^{2}$ is also transverse to $\mathcal{A}_{D}$ by Proposition 9.12. The closures of these curves are then transverse to $\mathcal{A}_{D}$ because they intersect $Y_{D} \backslash X_{D}$ in the curves $C_{P}$, these intersections are transverse by Theorem 8.7, and the curves $C_{P}$ are leaves of $\mathcal{A}_{D}$ by Proposition 9.12 .

Theorem 12.2 For any connected component $C$ of $P_{D}$ or $S_{D}^{2}$,

$$
[\bar{C}]^{2}=\chi(C) .
$$

For any component $C$ of $W_{D}$,

$$
[\bar{C}]^{2}=\frac{1}{3} \chi(C)
$$

Proof Let $C$ be a connected component of $P_{D}, S_{D}^{2}$, or $W_{D}$, and choose a tubular neighborhood $U \subset Y_{D}$ of $\bar{C}$ which is small enough that each point in $U \backslash \bar{C}$ represents a stable Abelian differential which has a unique shortest saddle connection joining distinct zeros (up to the hyperelliptic involution). We wish to define a map,

$$
\Phi: U \rightarrow\left(Q^{1} \bar{C}\right)^{*}
$$

where $Q^{1} \bar{C}$ is the restriction of $Q^{1} Y_{D}$ to $\bar{C}$.

Consider $p \in U$ representing the projective class of a stable Abelian differential $(X,[\omega])$. Let $I \subset X$ be the unique shortest saddle connection connecting distinct zeros, and let $(Y, \eta)$ be the stable Abelian differential obtained by collapsing $I$ as in Section 4.1. The projective class $(Y,[\eta])$ then represents a point of $\bar{C}$. Since $\omega$ and $\eta$ are both eigenforms for real multiplication, we have an isomorphism,

$$
T: \Omega^{1}(Y) \rightarrow \Omega^{1}(X),
$$

defined by $T(\eta)=\omega$. Define $S \in Q^{1}(Y)^{*}=\Omega^{1}(Y)^{-2}$ by

$$
S(v)=\left(\int_{I} T(\sqrt{v})\right)^{2},
$$

where the integral along $I$ is with respect to some choice of orientation of $I$. It doesn't matter which orientation we take for $I$ or which square root of $v$ we take, so $S$ is well-defined. Now define $\Phi$ by

$$
\Phi(p)=((Y,[\eta]), S) .
$$

For $p \in \bar{C}$, we define $\Phi(p)=(p, 0)$. Note that $\Phi$ takes leaves of $\mathcal{A}_{D}$ to fibers of $\left(Q^{1} \bar{C}\right)^{*}$.

Suppose that $C$ is a connected component of $P_{D}$. We claim that in this case, $\Phi$ is injective. To see this, let $q \in\left(Q^{1} \bar{C}\right)^{*}$ be represented by $(Y,[\eta]) \in \mathbb{P} \Omega \overline{\mathcal{M}}_{2}$ and 
$S \in Q^{1}(Y)^{*}$. This $(Y, \eta)$ is the one point union of two genus one differentials or cylinders:

$$
(Y, \eta)=\left(Y_{1}, \eta_{1}\right) \#\left(Y_{2}, \eta_{2}\right) .
$$

Normalize $\eta$ so that $S\left(\eta^{2}\right)=1$. If $\Phi(p)=q$, then $p$ is represented by the connected sum

$$
(X, \omega)=\left(Y_{1}, \eta_{1}\right) \#_{I}\left(Y_{2}, \eta_{2}\right),
$$

where $I \subset \mathbb{C}$ is the segment joining 0 to 1 . Thus $p$ is determined uniquely by $q$, so $\Phi$ is injective as claimed. When $C=S_{D}^{2}$, then $\Phi$ is injective by the same argument, using a self-connected sum in place of the connected sum operation above.

Recall that in Section 4.1, we defined the operation of splitting a double zero, which is inverse to the operation of collapsing a saddle connection, and associates to a sufficiently small segment $I \subset(Y, \eta)$ starting at the zero of $\eta$ the Abelian differential $(Y, \eta) \#_{I}$.

Suppose now that $C$ is a connected component of $W_{D}$. We claim that in this case, $\Phi$ is branched of order three along $\bar{C}$. Again, let $q \in\left(Q^{1} \bar{C}\right)^{*}$ be represented by $(Y, \eta) \in \mathbb{P} \Omega \overline{\mathcal{M}}_{2}$ and $S \in Q^{1}(Y)^{*}$, and normalize $\eta$ so that $S\left(\eta^{2}\right)=1$. This $(Y, \eta)$ is a stable Abelian differential with a double zero $z$. If $\Phi(p)=q$, then $p$ is represented by

$$
(X, \omega)=(Y, \eta) \#_{I}
$$

for some oriented segment $I$ starting at $z$ such that

$$
\int_{I} \eta=1
$$

There are at most three such segments because there are three positively oriented horizontal directions at the zero of $\eta$; therefore, $q$ has at most three preimages. If $S$ is small, then there is an embedded ball around $z$ with large radius. This means that there are three embedded segments starting at $z$ satisfying (12-2), and we can split along each of these segments. Thus any point in $Q^{1} \bar{C}$ sufficiently close to the zero section has exactly three preimages under $\Phi$, and the claim follows.

Now if $C$ is a connected component of $P_{D}$ or $S_{D}^{2}$, then we have seen that $U$ is homeomorphic to a neighborhood of the zero section in $Q^{1} \bar{C}$. Since $U$ is a tubular neighborhood of $\bar{C}, U$ is also homeomorphic to a neighborhood of the zero section in $N(\bar{C})$. Therefore,

$$
[\bar{C}]^{2}=\operatorname{deg} N(\bar{C})=\operatorname{deg} Q^{1} \bar{C} .
$$

Since $c_{1}\left(Q^{1} Y_{D}\right)=\left[\omega_{1}\right]$ by Corollary 9.10 ,

$$
[\bar{C}]^{2}=\operatorname{deg} Q^{1} \bar{C}=-\int_{C} \omega_{1}=\chi(C),
$$


as claimed.

Now suppose $C$ is a connected component of $W_{D}$. Regard $U$ as a neighborhood of the zero section in $N(\bar{C})$. By the above claim, we can choose $U$ so that $\Phi: U \rightarrow\left(Q^{1} \bar{C}\right)^{*}$ preserves fibers and is exactly three-to-one onto its image. For any line bundle $B \rightarrow \bar{C}$, let $\tau_{B} \in H^{2}(B, B \backslash \bar{C} ; \mathbb{R})$ be its Thom class. The Thom class $\tau_{\left(Q^{1} \bar{C}\right)^{*}}$ is represented by a 2 -form which is supported in $\Phi(U)$ which satisfies

$$
\int_{F} \tau_{\left(Q^{1} \bar{C}\right)^{*}}=1
$$

for each fiber $F$ of $\left(Q^{1} \bar{C}\right)^{*}$. For each fiber $F$ of $N(\bar{C})$, we have

thus

$$
\int_{F} \Phi^{*} \tau_{\left(Q^{1} \bar{C}\right)^{*}}=3
$$

It follows that

$$
\Phi^{*} \tau_{\left(Q^{1} \bar{C}\right)^{*}}=3 \tau_{N(\bar{C})} .
$$

$$
\begin{aligned}
{[\bar{C}]^{2} } & =\tau_{N(\bar{C})} \cdot[\bar{C}] \\
& =\frac{1}{3} \tau_{\left(Q^{1} \bar{C}\right)^{*} \cdot[\bar{C}]} \\
& =\frac{1}{3} \operatorname{deg}\left(Q^{1} \bar{C}\right)^{*} \\
& =\frac{1}{3} \chi(C) .
\end{aligned}
$$

Corollary 12.3 For any connected component $C$ of $W_{D}$, we have

$$
\int_{C} \omega_{2}=\frac{1}{3} \int_{C} \omega_{1}
$$

Proof By Proposition 12.1, we have

$$
[C]^{2}=\left.\operatorname{deg} T \mathcal{A}_{D}\right|_{C}=c_{1}\left(T \mathcal{A}_{D}\right) \cdot[C]=-\int_{C} \omega_{2} .
$$

By Theorem 12.2,

$$
[C]^{2}=-\frac{1}{3} \int_{C} \omega_{1}
$$

and the claim follows.

\section{Euler characteristic of $W_{D}^{\epsilon}$}

Cohomology of $\boldsymbol{Y}_{\boldsymbol{D}}$ From Theorem 8.11, the intersection pairing on $H^{2}\left(Y_{\boldsymbol{D}} ; \mathbb{Q}\right)$ is negative definite on the subspace $B$ generated by the fundamental classes of the curves 
$C_{P}$. By Corollary 9.11, the subspace $\left\langle\left[\omega_{1}\right],\left[\omega_{2}\right]\right\rangle \subset H^{2}\left(Y_{D} ; \mathbb{Q}\right)$ is orthogonal to $B$. Thus, if we let $J \subset H^{2}\left(Y_{D} ; \mathbb{Q}\right)$ be the orthogonal complement to $B \oplus\left\langle\left[\omega_{1}\right],\left[\omega_{2}\right]\right\rangle$, then we have the orthogonal direct sum

$$
H^{2}\left(Y_{D} ; \mathbb{Q}\right)=B \oplus\left\langle\left[\omega_{1}\right],\left[\omega_{2}\right]\right\rangle \oplus J
$$

Since

$$
B \oplus\left\langle\left[\omega_{1}\right],\left[\omega_{2}\right]\right\rangle \subset H^{1,1}\left(Y_{D} ; \mathbb{Q}\right),
$$

$J$ contains all of $H^{2,0}\left(Y_{D} ; \mathbb{Q}\right)$ and $H^{0,2}\left(Y_{D} ; \mathbb{Q}\right)$.

$\chi\left(\boldsymbol{W}_{\boldsymbol{D}}^{\boldsymbol{\epsilon}}\right)$ when $\mathbf{D}$ is not square We now calculate $\chi\left(W_{D}^{\epsilon}\right)$ when $D$ is not square. Until further notice, we will assume that $D$ is not square.

According to Corollary 8.12,

$$
\pi_{B}\left[\bar{W}_{D}\right]=\pi_{B}\left[\bar{P}_{D}\right] .
$$

Let $B_{D}=\pi_{B}\left[\bar{P}_{D}\right]$, and let $B_{D}^{\epsilon}=\pi_{B}\left[\bar{W}_{D}^{\epsilon}\right]$ for $\epsilon=1,2$. Since

$$
\left[\bar{W}_{D}\right]=\left[\bar{W}_{D}^{0}\right]+\left[\bar{W}_{D}^{1}\right]
$$

we have

$$
B_{D}^{0}+B_{D}^{1}=B_{D}
$$

Lemma 13.1 For any nonsquare $D$, we have

$$
\left(B_{D}\right)^{2}=-15 \chi\left(X_{D}\right) \text {. }
$$

Proof Since $W_{D}$ and $P_{D}$ are disjoint,

$$
\left[\bar{W}_{D}\right] \cdot\left[\bar{P}_{D}\right]=0 \text {. }
$$

Equation (13-2) follows directly from this together with the equations (11-3) and (11-9) for these fundamental classes.

Theorem 13.2 If $D$ is not square, then the fundamental class of $\bar{W}_{D}^{\epsilon}$ in $H^{2}\left(Y_{D} ; \mathbb{Q}\right)$ is

$$
\left[\bar{W}_{D}^{\epsilon}\right]=\frac{3}{4}\left[\omega_{1}\right]+\frac{9}{4}\left[\omega_{2}\right]+B_{D}^{\epsilon}+j
$$

for some $j \in J$.

Proof Since $W_{D}^{0}$ and $P_{D}$ are disjoint, we have

$$
\left[\bar{W}_{D}^{0}\right] \cdot\left[P_{D}\right]=0,
$$


and by (8-6), we have

$$
\left(B_{D}^{0}\right)^{2}=\left(B_{D}^{1}\right)^{2} .
$$

By (13-1) and Corollary 12.3, the fundamental classes of the $\bar{W}_{D}^{\epsilon}$ are of the form,

$$
\begin{aligned}
& {\left[\bar{W}_{D}^{0}\right]=a\left[\omega_{1}\right]+3 a\left[\omega_{2}\right]+B_{D}^{0}+j} \\
& {\left[\bar{W}_{D}^{1}\right]=\left(\frac{3}{2}-a\right)\left[\omega_{1}\right]+\left(\frac{9}{2}-3 a\right)\left[\omega_{2}\right]+B_{D}^{1}-j,}
\end{aligned}
$$

for some $a \in \mathbb{Q}$ and $j \in J$. In terms of $a$, (13-4) becomes

$$
10 a \chi\left(X_{D}\right)+\left(B_{D}^{0}\right)^{2}+B_{D}^{0} \cdot B_{D}^{1}=0 .
$$

From (13-2) and (13-5), we obtain

$$
\left(B_{D}^{0}\right)^{2}+B_{D}^{0} \cdot B_{D}^{1}=\frac{1}{2}\left(B_{D}^{0}+B_{D}^{1}\right)^{2}=\frac{1}{2}\left(B_{D}\right)^{2}=-\frac{15}{2} \chi\left(X_{D}\right) .
$$

Combining (13-6) and (13-7) yields

$$
a=\frac{3}{4}
$$

as desired.

Remark It seems likely that $j=0$, but we don't know how to prove this.

Corollary 13.3 If $D$ is not square, then

$$
\chi\left(W_{D}^{0}\right)=\chi\left(W_{D}^{1}\right)
$$

$\chi\left(W_{D}^{\epsilon}\right)$ when $\mathbf{D}$ is square We now turn to the calculation of $\chi\left(W_{d^{2}}^{\epsilon}\right)$. The idea is the same as the proof of Theorem 13.2, but the calculation is more complicated because of the presence of the curves $S_{d^{2}}^{i}$. We will restrict to the case $d>2$ because $W_{4}=\varnothing$. We start by calculating the intersections of various classes in $H^{2}\left(Y_{d^{2}} ; \mathbb{Q}\right)$.

Lemma 13.4 For any $d>2$, we have the following intersection numbers:

$$
\begin{aligned}
{\left[\omega_{1}\right] \cdot\left[\omega_{2}\right] } & =\frac{1}{72} d^{3} \sum_{r \mid d} \frac{\mu(r)}{r^{2}} \\
\pi_{B}\left[\bar{S}_{d^{2}}^{i}\right] \cdot \pi_{B}\left[\bar{P}_{d^{2}}\right] & =\left(-\frac{5}{24} d^{2}+\frac{1}{4} d\right) \sum_{r \mid d} \frac{\mu(r)}{r^{2}} \\
\left(\pi_{B}\left[\bar{S}_{d^{2}}^{i}\right]\right)^{2} & =-\frac{1}{12} d^{2} \sum_{r \mid d} \frac{\mu(r)}{r^{2}}
\end{aligned}
$$




$$
\begin{aligned}
\pi_{B}\left[\bar{S}_{d^{2}}^{1}\right] \cdot \pi_{B}\left[\bar{S}_{d^{2}}^{2}\right] & =-\frac{1}{2} d \sum_{r \mid d} \frac{\mu(r)}{r^{2}}+\frac{1}{2} \phi(d) \\
\pi_{B}\left[\bar{S}_{d^{2}}^{2}\right] \cdot \pi_{B}\left[\bar{W}_{d^{2}}\right] & =\left(-\frac{1}{8} d^{2}+\frac{1}{4} d\right) \sum_{r \mid d} \frac{\mu(r)}{r^{2}} \\
\left(\pi_{B}\left[\bar{W}_{d^{2}}\right]\right)^{2} & =\left(-\frac{5}{24} d^{3}+\frac{19}{24} d^{2}-\frac{3}{4} d\right) \sum_{r \mid d} \frac{\mu(r)}{r^{2}} \\
\left(\pi_{B}\left[\bar{P}_{d^{2}}\right]\right)^{2} & =\left(-\frac{5}{24} d^{3}+\frac{11}{24} d^{2}-\frac{1}{4} d\right) \sum_{r \mid d} \frac{\mu(r)}{r^{2}} \\
\pi_{B}\left[\bar{S}_{d^{2}}^{1}\right] \cdot \pi_{B}\left[\bar{W}_{d^{2}}\right] & =\left(-\frac{5}{24} d^{2}+\frac{3}{4} d\right) \sum_{r \mid d} \frac{\mu(r)}{r^{2}}-\frac{1}{2} \phi(d)
\end{aligned}
$$

Proof Equation (13-8) is $\chi\left(X_{d^{2}}\right)$, which is given in Theorem 2.12.

By Theorem 8.7, $\bar{P}_{d^{2}} \cap \bar{S}_{d^{2}}^{i}=\varnothing$. Thus $\left[\bar{P}_{d^{2}}\right] \cdot\left[\bar{S}_{d^{2}}^{i}\right]=0$, from which (13-9) follows.

By Theorem 12.2 and (10-11),

$$
\left[\bar{S}_{d^{2}}^{i}\right]^{2}=\chi\left(S_{d^{2}}^{i}\right)=-\frac{1}{12} d^{2} \sum_{r \mid d} \frac{\mu(r)}{r^{2}},
$$

from which (13-10) follows.

By Theorem 8.7,

$$
\left[\bar{S}_{d^{2}}^{1}\right] \cdot\left[\bar{S}_{d^{2}}^{2}\right]=-\frac{1}{2} \phi(d)
$$

Equation (13-11) follows.

By Theorem 8.7,

$$
\left[\bar{S}_{d^{2}}^{2}\right] \cdot\left[\bar{W}_{d^{2}}\right]=0
$$

Equation (13-12) follows.

By Theorem 12.2 and (10-6),

$$
\left[\bar{W}_{d^{2}}\right]^{2}=\frac{1}{3} \chi\left(W_{d^{2}}\right)=-\frac{1}{48} d^{2}(d-2) \sum_{r \mid d} \frac{\mu(r)}{r^{2}},
$$

from which (13-13) follows. Equation (13-14) is proved similarly.

By Corollary 8.12,

$$
\pi_{B}\left[\bar{S}_{d^{2}}^{1}\right] \cdot \pi_{B}\left[\bar{W}_{d^{2}}\right]=\pi_{B}\left[\bar{S}_{d^{2}}^{1}\right] \cdot \pi_{B}\left[\bar{P}_{d^{2}}\right]-\pi_{B}\left[\bar{S}_{d^{2}}^{1}\right] \cdot \pi_{B}\left[\bar{S}_{d^{2}}^{2}\right] .
$$

Then (13-15) follows from (13-9) and (13-11). 
Theorem 13.5 For any $d>2$, the fundamental class of $\bar{W}_{d^{2}}^{\epsilon}$ in $H^{2}\left(Y_{d^{2}} ; \mathbb{Q}\right)$ is given by

$$
\begin{aligned}
& {\left[\bar{W}_{d^{2}}^{0}\right]=\frac{3}{4}\left(1-\frac{1}{d}\right)\left[\omega_{1}\right]+\frac{9}{4}\left(1-\frac{1}{d}\right)\left[\omega_{2}\right]+\pi_{B}\left[\bar{W}_{d^{2}}^{0}\right]+j} \\
& {\left[\bar{W}_{d^{2}}^{1}\right]=\frac{3}{4}\left(1-\frac{3}{d}\right)\left[\omega_{1}\right]+\frac{9}{4}\left(1-\frac{3}{d}\right)\left[\omega_{2}\right]+\pi_{B}\left[\bar{W}_{d^{2}}^{1}\right]-j}
\end{aligned}
$$

for some $j \in J$.

Proof By (13-1), Corollary 11.3, and Corollary 12.3, the fundamental classes of the $\bar{W}_{d^{2}}^{\epsilon}$ are given by

$$
\begin{aligned}
& {\left[\bar{W}_{d^{2}}^{0}\right]=a\left[\omega_{1}\right]+3 a\left[\omega_{2}\right]+\pi_{B}\left[\bar{W}_{d^{2}}^{0}\right]+j} \\
& {\left[\bar{W}_{d^{2}}^{1}\right]=\left(\frac{3}{2}-\frac{3}{d}-a\right)\left[\omega_{1}\right]+\left(\frac{9}{2}-\frac{9}{d}-3 a\right)\left[\omega_{2}\right]+\pi_{B}\left[\bar{W}_{d^{2}}^{1}\right]-j}
\end{aligned}
$$

for some $a \in \mathbb{Q}$ and $j \in J$.

From Corollary 8.16, we have

$$
\begin{aligned}
\left(\pi_{B}\left[\bar{W}_{d^{2}}^{0}\right]\right)^{2} & =\left(\pi_{B}\left[\bar{W}_{d^{2}}^{1}\right]+\pi_{B}\left[\bar{S}_{d^{2}}^{2}\right]\right)^{2} \\
& =\left(\pi_{B}\left[\bar{W}_{d^{2}}\right]-\pi_{B}\left[\bar{W}_{d^{2}}^{0}\right]+\pi_{B}\left[\bar{S}_{d^{2}}^{2}\right]\right)^{2} .
\end{aligned}
$$

Using (13-10), (13-12), and (13-13), this simplifies to

$$
\begin{aligned}
\pi_{B}\left[\bar{W}_{d^{2}}\right] \cdot \pi_{B}\left[\bar{W}_{d^{2}}^{0}\right]+\pi_{B}\left[\bar{W}_{d^{2}}^{0}\right] \cdot \pi_{B}\left[\bar{S}_{d^{2}}^{2}\right] & \\
& =\left(-\frac{5}{48} d^{3}+\frac{11}{48} d^{2}-\frac{1}{8} d\right) \sum_{r \mid d} \frac{\mu(r)}{r^{2}} .
\end{aligned}
$$

From Corollary 8.12, we have

$$
\pi_{B}\left[W_{d^{2}}\right]+\pi_{B}\left[S_{d^{2}}^{2}\right]=\pi_{B}\left[P_{d^{2}}\right] .
$$

Multiplying (13-17) by $\pi_{B}\left[\bar{W}_{d^{2}}^{0}\right]$ and subtracting the result from (13-16), we obtain

$$
\pi_{B}\left[\bar{W}_{d^{2}}^{0}\right] \cdot \pi_{B}\left[\bar{P}_{d^{2}}\right]=\left(-\frac{5}{48} d^{3}+\frac{11}{48} d^{2}-\frac{1}{8} d\right) \sum_{r \mid d} \frac{\mu(r)}{r^{2}} .
$$

Since $\bar{W}_{d^{2}}^{0}$ and $\bar{P}_{d^{2}}$ are disjoint, we have

$$
\left[\bar{W}_{d^{2}}^{0}\right] \cdot\left[\bar{P}_{d^{2}}\right]=0 .
$$


Expanding, this becomes

$$
\left(\frac{5}{32} d^{3}-\frac{1}{6} d^{2}\right) a \sum_{r \mid d} \frac{\mu(r)}{r^{2}}+\pi_{B}\left[\bar{W}_{d^{2}}^{0}\right] \cdot \pi_{B}\left[\bar{P}_{d^{2}}\right]=0,
$$

which with (13-18) yields

$$
a=\frac{3}{4}\left(1-\frac{1}{d}\right) .
$$

Corollary 13.6 For any $d>2$ with $d \equiv 1(\bmod 2)$,

$$
\begin{aligned}
& \chi\left(W_{d^{2}}^{0}\right)=-\frac{1}{32} d^{2}(d-1) \sum_{r \mid d} \frac{\mu(r)}{r^{2}} \\
& \chi\left(W_{d^{2}}^{1}\right)=-\frac{1}{32} d^{2}(d-3) \sum_{r \mid d} \frac{\mu(r)}{r^{2}} .
\end{aligned}
$$

Once cylinder cusps As an application of the calculation of $\left[\bar{W}_{d^{2}}^{\epsilon}\right]$, we give formulas for the number of one-cylinder cusps of $W_{d^{2}}^{\epsilon}$. These formulas were established independently by Lelièvre and Royer in [47].

Theorem 13.7 For any $d>3$, the number of one-cylinder cusps of $W_{d^{2}}$ is

$$
\frac{1}{6} d^{2} \sum_{r \mid d} \frac{\mu(r)}{r^{2}}-\frac{1}{2} \phi(d)
$$

the number of one-cylinder cusps of $W_{d^{2}}^{0}$ is

$$
\frac{1}{24} d^{2} \sum_{r \mid d} \frac{\mu(r)}{r^{2}}
$$

and the number of one-cylinder cusps of $W_{d^{2}}^{1}$ is

$$
\frac{1}{8} d^{2} \sum_{r \mid d} \frac{\mu(r)}{r^{2}}-\frac{1}{2} \phi(d)
$$

Proof The one-cylinder cusps of $W_{d^{2}}$ are the points of the intersection,

$$
S_{d^{2}}^{1} \cap \bar{W}_{d^{2}} \text {. }
$$

When $d>3$ this intersection is transverse by Theorem 8.7 , so $\left[\bar{W}_{d^{2}}\right] \cdot\left[\bar{S}_{d^{2}}^{1}\right]$ is equal to the number of one-cylinder cusps of $W_{d^{2}}$. Similarly, $\left[\bar{W}_{d^{2}}^{\epsilon}\right] \cdot\left[\bar{S}_{d^{2}}^{1}\right]$ is equal to the number of one-cylinder cusps of $W_{d^{2}}^{\epsilon}$. 
Using (13-15), we obtain

$$
\left[\bar{W}_{d^{2}}\right] \cdot\left[\bar{S}_{d^{2}}^{1}\right]=\frac{1}{6} d^{2} \sum_{r \mid d} \frac{\mu(r)}{r^{2}}-\frac{1}{2} \phi(d),
$$

which implies (13-19).

Now let's calculate $\left[\bar{S}_{d^{2}}^{1}\right] \cdot\left[\bar{W}_{d^{2}}^{0}\right]$. By Corollary 8.16 , we have

$$
\begin{aligned}
& \pi_{B}\left[\bar{S}_{d^{2}}^{1}\right] \cdot \pi_{B}\left[\bar{W}_{d^{2}}^{0}\right] \\
& =\tau^{*}\left(\pi_{B}\left[\bar{S}_{d^{2}}^{1}\right]\right) \cdot \tau^{*}\left(\pi_{B}\left[\bar{W}_{d^{2}}^{0}\right]\right) \\
& =\pi_{B}\left[\bar{S}_{d^{2}}^{2}\right] \cdot \pi_{B}\left[\bar{W}_{d^{2}}^{1}\right]+\left(\pi_{B}\left[\bar{S}_{d^{2}}^{2}\right]\right)^{2} \\
& =\pi_{B}\left[\bar{S}_{d^{2}}^{2}\right] \cdot \pi_{B}\left[\bar{W}_{d^{2}}\right]-\pi_{B}\left[\bar{S}_{d^{2}}^{2}\right] \cdot \pi_{B}\left[\bar{W}_{d^{2}}^{0}\right]+\left(\pi_{B}\left[\bar{S}_{d^{2}}^{2}\right]\right)^{2} .
\end{aligned}
$$

Since $\bar{W}_{d^{2}}^{0}$ and $\bar{S}_{d^{2}}^{2}$ are disjoint, we have $\left[\bar{W}_{d^{2}}^{0}\right] \cdot\left[\bar{S}_{d^{2}}^{2}\right]=0$, and it follows that

$$
\pi_{B}\left[\bar{S}_{d^{2}}^{2}\right] \cdot \pi_{B}\left[\bar{W}_{d^{2}}^{0}\right]=\left(-\frac{1}{16} d^{2}+\frac{1}{16} d\right) \sum_{r \mid d} \frac{\mu(r)}{r^{2}} .
$$

Substituting (13-10), (13-12), and (13-23) into (13-22), we obtain

$$
\pi_{B}\left[\bar{S}_{d^{2}}^{1}\right] \cdot \pi_{B}\left[\bar{W}_{d^{2}}^{0}\right]=\left(-\frac{7}{48} d^{2}+\frac{3}{16} d\right) \sum_{r \mid d} \frac{\mu(r)}{r^{2}} .
$$

Equation (13-24) yields

$$
\left[\bar{S}_{d^{2}}^{1}\right] \cdot\left[\bar{W}_{d^{2}}^{0}\right]=\frac{1}{24} d^{2} \sum_{r \mid d} \frac{\mu(r)}{r^{2}},
$$

which implies (13-20). Equation (13-21) follows from (13-19) and (13-20).

\section{Siegel-Veech constants}

As an application of our results, we calculate the Siegel-Veech constants for counting cylinders on translation surfaces on the Teichmüller curves $W_{D}$ and $W_{D}^{\epsilon}$. This is basically a matter of plugging in our results into known formulas for these constants.

Given a translation surface $(X, \omega)$, let

$$
N((X, \omega), L)=\#\{\text { maximal cylinders of length at most } L \text { on }(X, \omega)\} \text {. }
$$


If $(X, \omega)$ lies on a Teichmüller curve, then Veech [77] showed

$$
N((X, \omega), L) \sim \frac{c}{\pi \operatorname{Area}(X, \omega)} L^{2} .
$$

The constant $c$, known as a Siegel-Veech constant, only depends on the Teichmüller curve on which $(X, \omega)$ lies. Let $c_{D}$ be and $c_{D}^{\epsilon}$ be the Siegel-Veech constants associated to $W_{D}$ and $W_{D}^{\epsilon}$ respectively.

Given a $W_{D}$-prototype $P=(a, b, c, \bar{q})$, define

$$
v(P)=\frac{c}{\operatorname{gcd}(a, c)}\left(1-\frac{a}{c} \lambda^{2}\right)\left(1+\frac{1}{\lambda^{2}}\right),
$$

where $\lambda=\lambda(P)$, the positive root of $a x^{2}+b x+c=0$.

Theorem 14.1 If $D$ is not square, then

$$
c_{D}=\frac{\sum_{P \in \mathcal{W}_{D}} v(P)}{-2 \chi\left(W_{D}\right)}, \quad \text { and } \quad c_{D}^{\epsilon}=\frac{\sum_{P \in \mathcal{W}_{D}^{\epsilon}} v(P)}{-2 \chi\left(W_{D}^{\epsilon}\right)},
$$

where $\mathcal{W}_{D}^{\epsilon}$ is the set of $W_{D}$-prototypes of spin invariant $\epsilon$.

Sketch of proof To fix notation, assume $W_{D}$ is connected as the proof is the same otherwise. Each cusp of $W_{D}$ corresponds to a $W_{D}$-prototype $P$, and above Theorem 4.9 , we associated to this cusp a surface $\left(X_{P}, \omega_{P}\right)$ on $W_{D}$ with a decomposition into two cylinders $C_{1}$ and $C_{2}$ (say $C_{1}$ is the short cylinder). The subgroup of the Veech group of $\left(X_{P}, \omega_{P}\right)$ (the stabilizer of this surface in $\left.\mathrm{SL}_{2} \mathbb{R}\right)$ which preserves the horizontal direction is generated by

$$
g=\left(\begin{array}{ll}
1 & t \\
0 & 1
\end{array}\right)
$$

where

$$
t=\frac{c}{\operatorname{gcd}(a, c)} .
$$

Let $i\left(C_{i}\right)$ be the order of the Dehn twist which $g$ induces on $C_{i}$. We have

and

$$
\begin{aligned}
& i\left(C_{1}\right)=-\frac{c}{\operatorname{gcd}(a, c)} \\
& i\left(C_{2}\right)=\frac{a}{\operatorname{gcd}(a, c)} .
\end{aligned}
$$

We have

$$
v(P)=\operatorname{Area}\left(X_{P}, \omega_{P}\right) \sum_{i} \frac{i\left(C_{i}\right)}{\operatorname{Area}\left(C_{i}\right)} .
$$


It follows from Theorem 6.5 of [30] that for any $(X, \omega) \in \Omega_{1} W_{D}$,

$$
N((X, \omega), L) \sim \frac{\sum_{P \in \mathcal{W}_{D}} v(P)}{\text { Area } W_{D}} L^{2},
$$

as desired.

We list the Siegel-Veech constants for $D<100$ in Table 2, using the convention $c_{D}=c_{D}^{0}$ if $D \equiv 1(\bmod 8)\left(c_{D}^{1}\right.$ is the Galois conjugate of $c_{D}^{0}$ as we prove below). From numerical calculations, as $D \rightarrow \infty$, the constants appear to converge to 10 , which by [23] is the Siegel-Veech constant for counting cylinders on a generic surface in $\Omega \mathcal{M}_{2}$ (2). It would be interesting to find a closed formula for $c_{D}$.

Arithmetic of Siegel-Veech constants According to [30], the Siegel-Veech constant $c$ in (14-1) lies in the trace field of the Veech group of $(X, \omega)$. We get more precise information in the case of the $W_{D}$ :

Theorem 14.2 Suppose $D$ is not square. If $D \not \equiv 1(\bmod 8)$, then $c_{D} \in \mathbb{Q}$. Otherwise, $c_{D}^{0}$ and $c_{D}^{1}$ are Galois conjugate elements of $\mathbb{Q}(\sqrt{D})$.

Proof First assume $D \not \equiv 1(\bmod 8)$. We have the involution $t$ on the set of $W_{D^{-}}$ prototypes, defined in Section 3. Actually, it was defined on $Y_{D}$-prototypes, but the definition works just as well in this case. This involution satisfies

$$
v(t(P))=v(P)^{\prime},
$$

where the prime denotes Galois conjugation. It follows that $c_{D}^{\prime}=c_{D}$ as desired.

Now suppose $D \equiv 1(\bmod 8)$. If it were true that $\epsilon(t(P))=\epsilon(P)+1-$ where $\epsilon$ is the spin invariant - then we would be done. This is not true, but we can modify $t$ so that it is. Define a bijection on the set of $W_{D}$-prototypes:

$$
s(P)= \begin{cases}P, & \text { if } a-b+c<0 \text { or } a \neq \equiv \quad(\bmod 2) \\ P+(0,0,0, \operatorname{gcd}(a, b, c)), & \text { otherwise }\end{cases}
$$

Then $t^{\prime}=s \circ t$ is also a bijection. One can check that it satisfies

and

$$
\epsilon\left(t^{\prime}(P)\right)=\epsilon(P)+1
$$

$$
v\left(t^{\prime}(P)\right)=v(P)^{\prime},
$$

from which the second claim follows. 


\begin{tabular}{|c|c|c|c|}
\hline$D$ & $c_{D}$ & $D$ & $c_{D}$ \\
\hline 5 & 25 & 53 & 228695 \\
\hline$J$ & $\overline{3}$ & 33 & $\overline{21021}$ \\
\hline 8 & 28 & 56 & 1796 \\
\hline & 3 & & $\overline{195}$ \\
\hline 12 & $\frac{26}{3}$ & 57 & $\frac{23693}{2352}+\frac{29}{784} \sqrt{57}$ \\
\hline 13 & 91 & 60 & 2158 \\
\hline & 9 & & 231 \\
\hline 17 & $\frac{221}{24}+\frac{1}{8} \sqrt{17}$ & 61 & $\frac{194651}{19305}$ \\
\hline 20 & $\frac{31}{3}$ & 65 & $\frac{52429}{5376}+\frac{113}{1792} \sqrt{65}$ \\
\hline 21 & 133 & 68 & 413 \\
\hline & $\overline{15}$ & & 39 \\
\hline 24 & 148 & 69 & $\underline{26611}$ \\
\hline 24 & 15 & 09 & 2805 \\
\hline 28 & $\frac{82}{9}$ & 72 & $\frac{18868}{1785}$ \\
\hline 29 & $\frac{377}{35}$ & 73 & $\frac{3285}{352}+\frac{23}{864} \sqrt{73}$ \\
\hline 32 & $\frac{190}{21}$ & 76 & $\frac{2822}{285}$ \\
\hline 33 & $\frac{473}{48}-\frac{11}{144} \sqrt{33}$ & 77 & $\frac{116699}{12597}$ \\
\hline 37 & $\frac{9139}{945}$ & 80 & $\frac{12631}{1254}$ \\
\hline 40 & $\begin{array}{c}945 \\
1924 \\
189\end{array}$ & 84 & $\frac{487}{51}$ \\
\hline 41 & $\frac{8897}{960}-\frac{23}{320} \sqrt{41}$ & 85 & $\frac{336821}{32319}$ \\
\hline 44 & $\frac{7682}{735}$ & 88 & $\frac{182236}{18837}$ \\
\hline 45 & $\frac{299}{33}$ & 89 & $\frac{702833}{68640}-\frac{831}{22880} \sqrt{89}$ \\
\hline 48 & $\frac{325}{33}$ & 92 & $\frac{204178}{21945}$ \\
\hline 52 & $\frac{1283}{135}$ & 93 & $\frac{2823449}{270963}$ \\
\hline
\end{tabular}

Table 2: Siegel-Veech constants for $W_{D}$

Applications to billiards Given a L-shaped polygon $P$ (or more generally a rational angled polygon), there is a construction called unfolding which produced a translation surface from $P$. To construct the unfolding of $P$, join four copies of $P$ to form a cross and glue opposite sides as in Figure 15. This yields a genus two translation surface $(X, \omega)$ with a single double zero. Closed billiards paths on $P$ unfold to closed geodesics on $(X, \omega)$, and each closed billiards path which is neither horizontal nor vertical is the image of exactly two closed geodesics on $U(P)$. Thus we obtain a twoto-one correspondence between closed paths of length $L$ on $P$ and on the unfolding. 
Recall that we defined in Section 1 a L-shaped polygon $P(D)$ for each real quadratic discriminant $D$, and we defined $N(P(D), L)$ to be the number of families of closed billiards paths of length at least $L$. From the above discussion, we obtain

$$
N(U(P(D)), L) \sim 2 N(P(D), L) .
$$

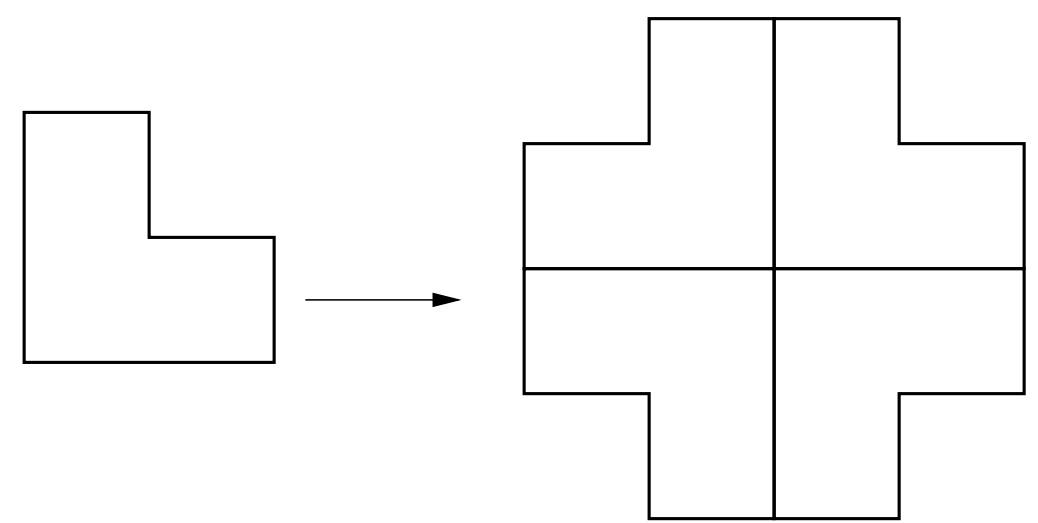

Figure 15: Unfolding a L-shaped polygon

Theorem 14.3 If $D$ is not square, then

$$
N(P(D), L) \sim c(D) \frac{1}{8 \pi \operatorname{Area}(P(D))} L^{2},
$$

where $c(D)=c_{D}$, if $D \neq \equiv 1(\bmod 8)$, and $c(D)=c_{D}^{(1+f) / 2}$ if $D \equiv 1(\bmod 8)$, where $f$ is the conductor of $D$.

Proof This follows directly from (14-1) and (14-2). There is an extra factor of 4 is because the unfolding has 4 times the area. To see that the unfolding has discriminant $D$ and spin invariant $\epsilon=(1+f) / 2$, note that the unfolding is $\mathrm{GL}_{2} \mathbb{R}$-equivalent to the surface $\left(X_{P}, \omega_{P}\right)$ associated to the $W_{D}$-prototype,

$$
P= \begin{cases}\left(1,-1, \frac{1-D}{4}, 0\right) ; & \text { if } D \text { is odd } \\ \left(1,0,-\frac{D}{4}, 0\right) ; & \text { if } D \text { is even }\end{cases}
$$

defined above Theorem 4.9. The spin invariant is then given by Theorem 4.10. 


\section{Lyapunov exponents}

Consider the rank $2 g$ bundle $\mathcal{H}_{1}(\mathbb{R}) \rightarrow \Omega_{1} \mathcal{M}_{g}$ whose fiber over the surface $(X, \omega)$ is $H_{1}(X ; \mathbb{R}) / \operatorname{Aut}(X, \omega)$. The action of the diagonal subgroup of $\mathrm{SL}_{2} \mathbb{R}$ lifts to a linear action on $\mathcal{H}_{1}(\mathbb{R})$. This action is called the Kontsevich-Zorich cocycle. The $2 g$ Lyapunov exponents of the Kontsevich-Zorich cocycle with respect to a finite, ergodic, $\mathrm{SL}_{2} \mathbb{R}$-invariant measure $\mu$ are of the form

$$
1=\lambda_{1}(\mu)>\cdots>\lambda_{g}(\mu)>0>-\lambda_{g}(\mu)>\cdots-\lambda_{1}(\mu)=-1 .
$$

The goal of this section is to prove the following unpublished theorem of Kontsevich and Zorich.

Theorem 15.1 If $\mu$ is any finite, ergodic, $\mathrm{SL}_{2} \mathbb{R}$-invariant measure on $\Omega_{1} \mathcal{M}_{2}$, then

$$
\lambda_{2}(\mu)= \begin{cases}1 / 3, & \text { if } \mu \text { is supported on } \Omega_{1} \mathcal{M}_{2}(2) ; \\ 1 / 2, & \text { if } \mu \text { is supported on } \Omega_{1} \mathcal{M}_{2}(1,1)\end{cases}
$$

The proof of Theorem 15.1 is based on a formula due to Kontsevich [42] for the sums of Lyapunov exponents in terms of some integrals over $\mathbb{P} \Omega \mathcal{M}_{g}$. Kontsevich states his formula for the Lyapunov exponents on the entire strata $\Omega_{1} \mathcal{M}_{g}(\mathbf{n})$, but his formula is equally valid for all ergodic, $\mathrm{SL}_{2} \mathbb{R}$-invariant probability measures on $\Omega_{1} \mathcal{M}_{2}$ and we will state it for these.

Let $E$ be the rank-two vector bundle over $\mathbb{P} \Omega \mathcal{M}_{2}$ obtained by pulling back $\Omega \mathcal{M}_{2}$ by $\mathbb{P} \Omega \mathcal{M}_{2} \rightarrow \mathcal{M}_{2}$, and let $L$ be the canonical sub-line-bundle whose fiber over $(X,[\omega])$ is $\mathbb{C} \omega$. Give $E$ the Hodge metric,

$$
h(\omega, \eta)=\int_{X} \omega \wedge \bar{\eta},
$$

and define two-forms on $\mathbb{P} \Omega \mathcal{M}_{2}$,

$$
\gamma_{1}=c_{1}(E, h) \quad \text { and } \quad \gamma_{2}=c_{1}(L, h) \text {. }
$$

For any $\mathrm{SL}_{2} \mathbb{R}$-invariant measure $\mu$ on $\Omega_{1} \mathcal{M}_{g}$, let $\pi_{*} \mu$ be the pushforward to $\mathbb{P} \Omega \mathcal{M}_{g}$, and let $\mathbb{P} \mu$ be the disintegration of $\pi_{*} \mu$ with respect to the foliation $\mathcal{F}$ of $\mathbb{P} \Omega \mathcal{M}_{g}$ by images of $\mathrm{SL}_{2} \mathbb{R}$-orbits. That is, $\mathbb{P} \mu$ is the unique transverse invariant measure such that the product of $\mathbb{P} \mu$ with the hyperbolic area measure on the leaves of $\mathcal{F}$ is $\pi_{*} \mu$. See Bourbaki [15] for a discussion of disintegration. 
Theorem 15.2 [42] For any finite ergodic, $\mathrm{SL}_{2} \mathbb{R}$-invariant measure $\mu$ on $\Omega_{1} \mathcal{M}_{2}$,

$$
\lambda_{1}(\mu)+\lambda_{2}(\mu)=\frac{\int_{\mathbb{P} \mu} \gamma_{1}}{\int_{\mathbb{P} \mu} \gamma_{2}} .
$$

Uniform distribution In order to evaluate (15-1), we will need the following weak uniform distribution result for Teichmüller curves. Given a space $X$ with an $\mathrm{SL}_{2} \mathbb{R}-$ action, let $M(X)$ be the space of finite $\mathrm{SL}_{2} \mathbb{R}$-invariant measures on $X$ with the weak* topology. Let $C(\mathbf{n}) \subset M\left(\Omega_{1} \mathcal{M}_{g}(\mathbf{n})\right)$ be the closed, convex cone spanned by the measures defined by hyperbolic area on those Teichmüller curves in $\Omega_{1} \mathcal{M}_{g}(\mathbf{n})$ which are generated by square-tiled surfaces. Similarly, let $C_{d^{2}} \subset\left(\Omega_{1} E_{d^{2}}(1,1)\right)$ be the closed, convex cone spanned by those Teichmüller curves in $\Omega_{1} E_{d^{2}}(1,1)$ generated by square-tiled surfaces.

Theorem 15.3 We have

and

$$
\begin{aligned}
\mu^{\prime}(\mathbf{n}) & \in C(\mathbf{n}) \\
\mu_{d^{2}}^{\prime} & \in C_{d^{2}},
\end{aligned}
$$

where the measures on the left are the period measures on these strata defined in Section 4.

Let $\eta_{m}(\mathbf{n})$ and $\eta_{m}[d]$ be the measures on $\Omega \mathcal{M}_{g}(\mathbf{n})$ and $\Omega E_{d^{2}}(1,1)$ defined by putting $\delta$-masses of equal weight on the square-tiled surfaces with at most $m$ squares, normalized to have the same volumes as $\mu^{\prime}(\mathbf{n})$ and $\mu_{d^{2}}^{\prime}$ respectively, which are finite by Theorem 4.2. Let $\eta_{m}^{\prime}(\mathbf{n})$ and $\eta_{m}^{\prime}[d]$ be the projections to $\Omega_{1} \mathcal{M}_{g}(\mathbf{n})$ and $\Omega_{1} E_{d^{2}}(1,1)$. The following lemma is well-known.

Lemma 15.4 We have

$$
\begin{aligned}
& \lim _{m \rightarrow \infty} \eta_{m}^{\prime}(\mathbf{n})=\mu^{\prime}(\mathbf{n}) \\
& \lim _{m \rightarrow \infty} \eta_{m}^{\prime}[d]=\mu_{d^{2}}^{\prime}
\end{aligned}
$$

in the weak* topology on measures.

Proof We will prove only the first statement for concreteness, the other having essentially the same proof. Let $\mu^{\prime \prime}(\mathbf{n})$ be the restriction of $\mu^{\prime}(\mathbf{n})$ to $\Omega_{\leq 1} \mathcal{M}_{g}(\mathbf{n})$, and let

$$
\eta_{m}^{\prime \prime}(\mathbf{n})=\left(t_{1 / \sqrt{m}}\right)_{*} \eta_{m}(\mathbf{n}),
$$


where $t_{r}$ is the multiplication-by- $r$ map on $\Omega \mathcal{M}_{g}$. Since $\mu^{\prime \prime}(\mathbf{n})$ and $\eta_{m}^{\prime \prime}(\mathbf{n})$ project to $\mu^{\prime}(\mathbf{n})$ and $\eta_{m}^{\prime}(\mathbf{n})$ respectively, it is enough to show that

$$
\lim _{m \rightarrow \infty} \eta_{m}^{\prime \prime}(\mathbf{n})=\mu^{\prime \prime}(\mathbf{n}) \text {. }
$$

This is easy to see in the period coordinates on $\Omega \mathcal{M}_{g}(\mathbf{n})$ (described in Section 4). In period coordinates, the square-tiled surfaces are exactly the points on the integer lattice, so $\eta_{m}^{\prime \prime}(\mathbf{n})$ consists of $\delta$-masses of uniform weight on points of norm less than one on the $\mathbb{Z} / \sqrt{m}$ lattice. Since these lattices have mesh approaching zero, the measures converge to the uniform measure which is just $\mu^{\prime \prime}(\mathbf{n})$ in period coordinates.

Proof of Theorem 15.3 The set of square-tiled surfaces is $\mathrm{SL}_{2} \mathbb{Z}$-invariant, so the measure $\eta_{m}^{\prime}(\mathbf{n})$ is also $\mathrm{SL}_{2} \mathbb{Z}$-invariant. Thus we can define

$$
v_{m}(\mathbf{n})=\int_{\mathrm{SL}_{2} \mathbb{R} / \mathrm{SL}_{2} \mathbb{Z}} \eta_{m}^{\prime}(\mathbf{n}) d \rho \in M\left(\Omega_{1} \mathcal{M}_{g}(\mathbf{n})\right),
$$

where $\rho$ is Haar measure on $\mathrm{SL}_{2} \mathbb{R}$. The measure $v_{m}(\mathbf{n})$ is $\mathrm{SL}_{2} \mathbb{R}$-invariant and supported on finitely many Teichmüller curves generated by square-tiled surfaces, so $v_{m}(\mathbf{n})$ lies in the cone $C(\mathbf{n})$.

Let $\Delta \subset \mathrm{SL}_{2} \mathbb{R}$ be a fundamental domain for $\mathrm{SL}_{2} \mathbb{Z}$. Given a compactly supported, continuous function $f$ on $\Omega_{1} \mathcal{M}_{g}(\mathbf{n})$, we have

$$
\begin{aligned}
\lim _{m \rightarrow \infty} \int_{\Omega_{1} \mathcal{M}_{g}(\mathbf{n})} f d v_{m}(\mathbf{n}) & =\lim _{m \rightarrow \infty} \int_{A \in \Delta \Omega_{1} \mathcal{M}_{g}(\mathbf{n})} f \circ A d \eta_{m}^{\prime}(\mathbf{n}) d \rho \\
& =\int_{A \in \Delta}\left(\lim _{m \rightarrow \infty} \int_{\Omega_{1} \mathcal{M}_{g}(\mathbf{n})} f \circ A d \eta_{m}^{\prime}(\mathbf{n})\right) d \rho \\
& =\int_{A \in \Delta \Omega_{1} \mathcal{M}_{g}(\mathbf{n})} f \circ A d \mu^{\prime}(\mathbf{n}) d \rho \\
& =\int_{A \in \Delta \Omega_{1} \mathcal{M}_{g}(\mathbf{n})} f d \mu^{\prime}(\mathbf{n}) d \rho \\
& =\operatorname{vol}\left(\operatorname{SL}_{2} \mathbb{R} / \mathrm{SL}_{2} \mathbb{Z}\right) \int_{\Omega_{1} \mathcal{M}_{g}(\mathbf{n})} f d \mu^{\prime}(\mathbf{n}),
\end{aligned}
$$

where the second equality follows from the Dominated Convergence Theorem; the third equality follows from Lemma 15.4; and the fourth equality uses the $\mathrm{SL}_{2} \mathbb{R}$-invariance of $\mu^{\prime}(\mathbf{n})$. It follows that $\mu^{\prime}(\mathbf{n}) \in C(\mathbf{n})$. 
The Siegel-Veech transform Given any translation surface $(X, \omega)$ with an oriented saddle connection $I$, we associate the complex number

$$
v(I)=\int_{I} \omega
$$

Let $V(X, \omega)$ be the collection of vectors in $\mathbb{C}$ associated with saddle connections on $(X, \omega)$, and for each $v \in V(X, \omega)$, let $n(v)$ be the number of saddle connections $I$ satisfying (15-2).

For any nonnegative measurable function $f: \mathbb{C} \rightarrow \mathbb{R}$, the Siegel-Veech transform $\hat{f}: \Omega_{1} \mathcal{M}_{g}(\mathbf{n}) \rightarrow \mathbb{R}$ is defined by

$$
\hat{f}(X, \omega)=\sum_{v \in V(X, \omega)} n(v) f(v) .
$$

Theorem 15.5 [80] Given any ergodic, $\mathrm{SL}_{2} \mathbb{R}$-invariant measure $\mu$ on $\Omega_{1} \mathcal{M}_{g}(\mathbf{n})$, we have

$$
\frac{1}{\operatorname{vol} \mu} \int_{\Omega_{1} \mathcal{M}_{g}(\mathbf{n})} \hat{f} d \mu=c_{\mathrm{sc}}(\mu) \int_{\mathbb{R}^{2}} f .
$$

The Siegel-Veech constant $c_{\mathrm{sc}}(\mu)$ depends only on the measure $\mu$.

If $\mu$ is supported on a Teichmüller curve $C$, we will also write $c_{\mathrm{sc}}(C)$ for $c_{\mathrm{sc}}(\mu)$. It follows from [80] that for any $(X, \omega)$ on a Teichmüller curve $C$,

$$
N_{\mathrm{sc}}((X, \omega), L) \sim c_{\mathrm{sc}}(C) \frac{\pi}{\operatorname{Area}(X, \omega)} L^{2},
$$

where $N_{\mathrm{sc}}$ is the counting function for saddle connections.

Bounded Siegel-Veech constants We now show that Siegel-Veech constants are uniformly bounded over Teichmüller curves of a given genus.

Theorem 15.6 There is a uniform bound,

$$
c_{\mathrm{sc}}(C) \leq D
$$

where $C$ ranges over all Teichmüller curves in $\Omega \mathcal{M}_{g}$, and $D$ only depends on the genus.

This follows easily from the following results. 
Theorem 15.7 [50; 21] Given any compact subset $K \subset \Omega \mathcal{M}_{g}(\mathbf{n})$, there is a constant $c(K)$ such that for any $(X, \omega) \in K$,

$$
N_{\text {sc }}((X, \omega), L)<c(K) L^{2} .
$$

Theorem 15.8 Given any connected component $S$ of a stratum $\Omega_{1} \mathcal{M}_{g}(\mathbf{n})$, there is a compact subset $K \subset S$ which intersects each $\mathrm{SL}_{2} \mathbb{R}$-orbit $S$.

Theorem 15.8 is a corollary of the main result in Athreya's thesis [3].

Proof of Theorem 15.6 For each connected component $S$ of a stratum, choose a $K$ as in Theorem 15.8. Then for every Teichmüller curve $C \subset S$, we have $c_{\mathrm{sc}}(C) \leq c(K)$ by Theorem 15.7. Since there are only finitely many connected components of strata in each genus by [43], we obtain a bound depending only on the genus.

Let $K_{\epsilon} \subset \Omega_{1} \mathcal{M}_{g}(\mathbf{n})$ be the locus of translation surfaces which have no saddle connection of length less than $\epsilon$, and let $\widetilde{K}_{\epsilon}$ be the complement of $K_{\epsilon}$.

Corollary 15.9 For any Teichmüller curve $C \subset \Omega_{1} \mathcal{M}_{g}(\mathbf{n})$, we have

$$
\frac{\operatorname{vol}\left(C \cap \tilde{K}_{\epsilon}\right)}{\operatorname{vol}(C)}<D \epsilon^{2},
$$

for some constant $D$ depending only on the genus $g$.

Proof Let $\chi_{\epsilon}$ and $\chi_{\tilde{K}_{\epsilon}}$ be the indicator functions of the disk of radius $\epsilon$ in $\mathbb{C}$ and $\tilde{K}_{\epsilon} \subset \Omega_{1} \mathcal{M}_{g}(\mathbf{n})$ respectively. We have

$$
\widehat{\chi}_{\epsilon} \geq \chi_{\tilde{K}_{\epsilon}} .
$$

Thus

$$
\frac{\operatorname{vol}\left(C \cap \tilde{K}_{\epsilon}\right)}{\operatorname{vol}(C)} \leq \frac{1}{\operatorname{vol}(C)} \int_{C} \hat{\chi}_{\epsilon}=c_{\mathrm{sc}}(C) \pi \epsilon^{2} \leq D \pi \epsilon^{2},
$$

where $D$ is the constant of Theorem 15.6.

Extension of $\mathcal{F}_{\boldsymbol{D}}$ to $\boldsymbol{Y}_{\boldsymbol{D}}$ We equipped the foliation $\mathcal{F}_{D}$ of $X_{D}$ by $\mathrm{SL}_{2} \mathbb{R}$-orbits with a transverse invariant measure $\mathbb{P} \mu_{D}^{\prime}$, so we can integrate 2 -forms over $\mathcal{F}_{D}$ to obtain a closed current $\left\langle\mathcal{F}_{D}\right\rangle$ on $X_{D}$. The foliation $\mathcal{F}_{D}$ does not extend to a foliation of $Y_{D}$. In fact, $\mathcal{F}_{D}$ has isolated singularities at the points $c_{P}$ as well as along the one-dimensional loci in the curves $C_{P}$ consisting of forms whose horizontal foliation has two cylinders. Nevertheless, the current $\left\langle\mathcal{F}_{D}\right\rangle$ extends to a closed current on $Y_{D}$. We prove the following two theorems in [7]. 
Theorem 15.10 The foliation $\mathcal{F}_{D}$ defines a closed current $\left\langle\mathcal{F}_{D}\right\rangle$ on $Y_{D}$, defined by

$$
\left\langle\mathcal{F}_{D}\right\rangle(\omega)=\int_{\mathcal{F}_{D}} \omega
$$

for each $C^{\infty} 2-$ form $\omega$ on $Y_{D}$.

Let $\left[\mathcal{F}_{D}\right]$ be the cohomology class in $H^{2}\left(Y_{D} ; \mathbb{R}\right)$ defined by $\left\langle\mathcal{F}_{D}\right\rangle$.

Theorem 15.11 For any component $C$ of $\bar{W}_{D}, \bar{P}_{D}$, or $\bar{S}_{D}^{2}$, we have

$$
[C] \cdot\left[\mathcal{F}_{D}\right]=0 .
$$

Remark In fact, we only prove the nonsquare discriminant cases of these theorems in [7]. The square discriminant cases can be proved by the same methods, but they are not needed here.

Proof of Theorem 15.1 Consider the pullbacks of the bundles $E$ and $L$ over $\mathbb{P} \Omega \mathcal{M}_{2}$ by $\pi: X_{D} \rightarrow \mathbb{P} \Omega \mathcal{M}_{2}$. We have

$$
\pi^{*} E=\Omega^{1} X_{D} \oplus \Omega^{2} X_{D}
$$

and

$$
\pi^{*} L=\Omega^{1} X_{D}
$$

Proposition 2.8 implies

and

$$
\begin{aligned}
& \pi^{*} \gamma_{1}=\omega_{1}+\omega_{2} \\
& \pi^{*} \gamma_{2}=\omega_{1} .
\end{aligned}
$$

Let $\mu$ be an ergodic $\mathrm{SL}_{2} \mathbb{R}$-invariant measure on $\Omega_{1} \mathcal{M}_{2}$ whose support is not one of the strata $\Omega_{1} \mathcal{M}_{2}(2)$ or $\Omega_{1} \mathcal{M}_{2}(1,1)$. By McMullen's classification of ergodic, invariant measures, each such $\mu$ is supported on one of the eigenform loci $\Omega_{1} E_{D}$. Then $\mathbb{P} \mu$ is a transverse, invariant measure to the foliation $\mathcal{F}_{D}$ of $X_{D}$, and (15-1) becomes

$$
\lambda_{2}(\mu)=\frac{\int_{\mathbb{P} \mu} \omega_{2}}{\int_{\mathbb{P} \mu} \omega_{1}} .
$$

If the ergodic, invariant measure $\mu$ is supported on a connected component $C$ of $W_{D}$, then $\lambda_{2}(\mu)=1 / 3$ by (15-4) and Corollary 12.3 .

Now suppose $\mu$ is supported on a Teichmüller curve $C \subset \mathbb{P} \Omega \mathcal{M}_{2}(1,1)$, so $C$ is either the decagon curve $D_{10}$ or a component of $W_{d^{2}}[n]$. By Corollary 10.3, we have

$$
\left[\bar{W}_{D}\right]-\left[\bar{P}_{D}\right]+\left[\bar{S}_{D}^{2}\right]=-\left[\omega_{1}\right]+2\left[\omega_{2}\right] .
$$


Since $\bar{C}$ is disjoint from each of the three curves on the left, pairing $[\bar{C}]$ with both sides of (15-5) yields $\lambda_{2}(\mu)=1 / 2$. If $\mu$ is the period measure $\mu_{D}^{\prime}$ on $E_{D}$, then

$$
\int_{\mathbb{P} \mu_{D}^{\prime}} \omega_{i}=\left[\mathcal{F}_{D}\right]\left(\omega_{i}\right)
$$

(see Theorem 8.7 of [7]). Then we obtain $\lambda_{2}\left(\mu_{D}^{\prime}\right)=1 / 2$ by pairing $\left[\mathcal{F}_{D}\right]$ with both sides of (15-5) and applying Theorem 15.11.

It remains to deal with the measures $\mu^{\prime}(\mathbf{n})$ on the strata $\Omega_{1} \mathcal{M}_{2}(\mathbf{n})$. Given a point $p \in \mathbb{P} \Omega \mathcal{M}_{2}$, let $L$ be the leaf of $\mathcal{F}$ through $p$. Define

$$
f(p)=\frac{\left.\gamma_{1}\right|_{L}}{\left.\gamma_{2}\right|_{L}}
$$

This is a well-defined real number because it is the ratio of top-degree forms.

Lemma 15.12 As a function on $\mathbb{P} \Omega \mathcal{M}_{2}$, we have

$$
1 \leq f \leq 2 \text {. }
$$

Proof Since $f$ is continuous, it suffices to prove this bound for every Teichmüller curve $C \subset \mathbb{P} \Omega \mathcal{M}_{2}$. The curve $C$ lies on some Hilbert modular surface $X_{D}$. Let

$$
\widetilde{C} \subset \mathbb{H} \times \mathbb{M}
$$

be a connected component of the inverse image of $C$ in the universal cover of $X_{D}$, and let $\pi_{i}: \widetilde{C} \rightarrow \mathbb{U}$ be the two projections. Since $C$ is transverse to the vertical foliation $\mathcal{A}_{D}$ of $X_{D}$, the first projection $\pi_{1}$ is a conformal isomorphism of $\widetilde{C}$ with $\mathbb{H}$. By the Schwartz lemma, the projection $\pi_{2}$ is a contraction with respect to the hyperbolic metrics. Since $\omega_{i}$ is the pullback of the hyperbolic area form on $\mathbb{U}$ by $\omega_{i}$, we have

$$
0 \leq \frac{\left.\omega_{2}\right|_{C}}{\left.\omega_{1}\right|_{C}} \leq 1
$$

The claim follows, since

$$
\left.f\right|_{C}=\frac{\left.\omega_{1}\right|_{C}+\left.\omega_{2}\right|_{C}}{\left.\omega_{1}\right|_{C}}
$$

We need to show that

$$
\frac{1}{\operatorname{vol} \mu^{\prime}(\mathbf{n})} \int_{\Omega_{1} \mathcal{M}_{2}(\mathbf{n})} f d \mu^{\prime}(\mathbf{n})= \begin{cases}1 / 3 ; & \text { if } \mathbf{n}=(2) ; \\ 1 / 2 ; & \text { if } \mathbf{n}=(1,1),\end{cases}
$$

where we regard $f$ as a function on $\Omega_{1} \mathcal{M}_{2}(\mathbf{n})$ by pulling it back from the projectivization. Let $\left\{v_{m}\right\}$ be a sequence of measures (provided by Theorem 15.3) supported 
on Teichmüller curves in $\Omega_{1} \mathcal{M}_{2}(\mathbf{n})$ and converging to $\mu^{\prime}(\mathbf{n})$. Since the average of $f$ over any Teichmüller curve $C$ is either $1 / 2$ or $1 / 3$, depending on the stratum in which $C$ lies, it is enough to show that

$$
\frac{1}{\operatorname{vol} v_{m}} \int f d v_{m} \rightarrow \frac{1}{\operatorname{vol} \mu^{\prime}(\mathbf{n})} \int f d \mu^{\prime}(\mathbf{n}) .
$$

Recall that we defined $K_{\epsilon}$ to be the locus of translation surfaces with no saddle connection shorter than $\epsilon$. Since $K_{\epsilon}$ is compact (as is shown in [40]), vol $\Omega_{1} \mathcal{M}_{2}(\mathbf{n})<\infty$, and the $K_{\epsilon}$ exhaust $\Omega_{1} \mathcal{M}_{2}(\mathbf{n})$ as $\epsilon \rightarrow 0$, we can choose for any $\delta>0$ an $\epsilon$ small enough that

$$
\frac{\mu^{\prime}(\mathbf{n})\left(\tilde{K}_{\epsilon}\right)}{\operatorname{vol} \mu^{\prime}(\mathbf{n})}<\frac{\delta}{2}
$$

By Corollary 15.9, we can also choose $\epsilon$ small enough that

$$
\frac{v_{m}(\mathbf{n})\left(\tilde{K}_{\epsilon}\right)}{\operatorname{vol} v_{m}(\mathbf{n})}<\frac{\delta}{2} .
$$

Let $g_{\epsilon}$ be a continuous, compactly supported function on $\Omega_{1} \mathcal{M}_{2}(\mathbf{n})$ such that $g \equiv 1$ on $K_{\epsilon}$, and $0 \leq g \leq 1$. We have

$$
\begin{aligned}
\lim _{m \rightarrow \infty} \mid & \left|\frac{1}{\operatorname{vol} v_{m}} \int f d v_{m}-\frac{1}{\operatorname{vol} \mu^{\prime}(\mathbf{n})} \int f d \mu^{\prime}(\mathbf{n})\right| \\
& \leq \lim _{m \rightarrow \infty}\left|\frac{1}{\operatorname{vol} v_{m}} \int g_{\epsilon} f d v_{m}-\frac{1}{\operatorname{vol} \mu^{\prime}(\mathbf{n})} \int g_{\epsilon} f d \mu^{\prime}(\mathbf{n})\right| \\
& +\frac{\mu^{\prime}(\mathbf{n})\left(\tilde{K}_{\epsilon}\right)}{\operatorname{vol} \mu^{\prime}(\mathbf{n})}+\frac{v_{m}(\mathbf{n})\left(\tilde{K}_{\epsilon}\right)}{\operatorname{vol} v_{\epsilon}(\mathbf{n})} \\
& \leq \delta
\end{aligned}
$$

since the limit of the first term is zero by Theorem 15.3, and the other two terms are bounded by (15-6) and (15-7).

This completes the proof of Theorem 15.1. Note that the last part of the proof applies to the $\Omega_{1} E_{d^{2}}$, so we can prove Theorem 15.1 for these spaces without appealing to Theorem 15.10 and Theorem 15.11.

\section{Appendix A Normal varieties}

In this section, we record standard facts about normality for algebraic varieties and analytic spaces which we use in this paper. We will consider all of our algebraic varieties to be over $\mathbb{C}$. 
Normal algebraic varieties A point $p$ on an algebraic variety $X$ is said to be normal if the local ring $\mathcal{O}_{p}$ of $X$ at $p$ is an integral domain which is integrally closed in its field of fractions. A variety $X$ is normal if it is normal at each of its points.

A normalization of a variety $X$ is a normal variety $Y$ together with a finite surjective morphism $\pi: Y \rightarrow X$. More generally (following [65]), if $X$ is an irreducible variety, and if $L$ is a finite algebraic extension of $K(X)$, then a normalization of $X$ in $L$ is a normal variety $Y$ with function field $K(Y)=L$, together with a finite surjective morphism $\pi: Y \rightarrow X$ such that $\pi^{*}: K(X) \rightarrow K(Y)=L$ is the given inclusion of $K(X)$ in $L$. If $L=K(X)$, this is just the usual normalization of $X$.

Theorem A.1 [65, Theorem III:8.3] For any irreducible variety $X$ and finite algebraic extension $L$ of $K(X)$, there is a normalization of $X$ in $L$, and any two such normalizations are equivalent.

Normalization is also characterized by a universal property. In the case, where $L=$ $K(X)$, this is an exercise in [33, p 91].

Theorem A.2 The normalization $\pi: Y \rightarrow X$ of $X$ in $L=K(Y)$ has the following universal property. Let $Z$ be a normal variety with a dominant morphism $q: Z \rightarrow X$, and let $r: K(Y) \rightarrow K(Z)$ be an isomorphism such that $r \circ \pi^{*}=q^{*}$ as inclusions $K(X) \rightarrow K(Z)$. Then there is a unique morphism $s: Z \rightarrow Y$ such that $s^{*}=r$ and $\pi \circ s=q$.

We will also have use for the following theorems:

Theorem A.3 [65, III:8.4] The normalization of a projective variety in a finite algebraic extension is projective.

Theorem A.4 (Zariski's Main Theorem [33]) Let $f: X \rightarrow Y$ be a birational morphism of projective varieties, and assume that $Y$ is normal. Then for every $y \in Y$, the fiber $f^{-1}(y)$ is connected.

Theorem A.5 (Zariski [83, Theorem VIII.32]) If $X$ is a normal variety, $p \in X$, and $\widehat{\mathcal{O}}_{p}$ is the completion of $\mathcal{O}_{p}$, then $\widehat{\mathcal{O}}_{p}$ is an integrally closed integral domain. 
Normal analytic spaces A complex analytic space is a ringed space which is locally modeled on analytic subvarieties of $\mathbb{C}^{n}$. We start by recalling some basic notions about complex analytic spaces. See Gunning and Rossi [29] and Gunning [28] for details.

Consider the set of pairs $(U, X)$, where $U \subset \mathbb{C}^{n}$ is a neighborhood of some point $p$, and $X \subset U$ is an analytic subvariety of $U$. We consider two such pairs to be equivalent if the varieties agree on some common neighborhood of $p$. An equivalence class of such pairs is called a germ on an analytic variety at $p$. A germ is considered to be irreducible if it contains no proper subgerms.

Theorem A.6 [28, p 11] Any germ of an analytic subvariety at $p$ can be written uniquely as the union of finitely many irreducible germs of analytic varieties at $p$.

Given a point $p \in X$, an analytic subvariety of an open subset of $\mathbb{C}^{n}$, a branch of $X$ through $p$ is an irreducible subgerm of the germ of $X$ at $p$. Informally, a branch of $X$ through $p$ is a connected component of $U \cap\left(X \backslash X_{\text {sing }}\right)$, where $U$ is a small and sufficiently regular neighborhood of $p$, and $X_{\text {sing }}$ is the singular set of $X$. Given a point $p$ in an analytic space $X$, let $\mathcal{O}_{p}$ be the local ring of germs of holomorphic functions on $X$ at $p$. Just as for algebraic varieties, we say that $p$ is a normal point of $X$ if $\mathcal{O}_{p}$ is an integrally closed integral domain. We say that $X$ is normal if each of its points is normal.

There is also a geometric characterization of normality. Following [81], we say that a function $f$ on an open subset $V$ of an analytic variety $X$ is weakly holomorphic if there is a subvariety $V^{\prime} \subset V$ with the following properties:

- $V^{\prime}$ is nowhere dense in $V$, and $V_{\text {sing }} \subset V^{\prime}$.

- $f$ is holomorphic in $V \backslash V^{\prime}$.

- $f$ is locally bounded in $V$.

It follows from [81, Theorem 4.10I] that an analytic space $X$ is weakly holomorphic at $p$ if and only if every germ of a weakly holomorphic function at $p$ is in fact holomorphic.

A normalization of an analytic space $X$ is a normal analytic variety $Y$ together with a holomorphic map $p: Y \rightarrow X$ such that the following holds:

- $\quad p: Y \rightarrow X$ is proper and has finite fibers.

- If $X_{\text {sing }}$ is the singular set of $X$, and $A=p^{-1}\left(X_{\text {sing }}\right)$, then $Y \backslash A$ is dense in $Y$, and $p: Y \backslash A \rightarrow X$ is biholomorphic onto $X \backslash X_{\text {sing }}$. 
Theorem A.7 [45, p 38] Every analytic space has a unique normalization.

Theorem A.8 [45, $\mathrm{p}$ 37] If $\pi: Y \rightarrow X$ is the normalization of $X$, then for each $p \in X$, the map $\pi$ induces a bijective correspondence between the points of $\pi^{-1}(p)$ and the branches of $X$ through $p$.

Theorem A.9 If $X$ is an algebraic variety, and $\pi: Y \rightarrow X$ is the normalization of $X$ as an algebraic variety then it is also the normalization of $X$ as an analytic space.

Proof By the definition of the normalization of an analytic space, we need only to show that $Y$ is normal as an analytic space. For this proof, write $\mathcal{O}_{p}^{\text {an }}$ for the local ring of germs of holomorphic functions on $X$ at $p$, to differentiate from the local ring of $\mathcal{O}_{p}$ of algebraic functions at $p$. Let $\widehat{\mathcal{O}}_{p}$ be the completion of $\mathcal{O}_{p}$.

Let $f$ in the quotient field $K\left(\mathcal{O}_{p}^{\text {an }}\right)$ be integral over $\mathcal{O}_{p}^{\text {an }}$. Since $\mathcal{O}_{p}^{\text {an }} \subset \widehat{\mathcal{O}}_{p}$, it follows that $f$ is integral over $\widehat{\mathcal{O}}_{p}$. Since $\widehat{\mathcal{O}}_{p}$ is integrally closed by Theorem A.5, it follows that $f \in \widehat{\mathcal{O}}_{p}$. Then $f \in \mathcal{O}_{p}^{\text {an }}$ because $K\left(\mathcal{O}_{p}^{\text {an }}\right) \cap \widehat{\mathcal{O}}_{p}=\mathcal{O}_{p}^{\text {an }}$ in $K\left(\widehat{\mathcal{O}}_{p}\right)$.

A resolution of an analytic space $X$ is a complex manifold $Y$ together with a proper analytic map $\pi: Y \rightarrow X$ such that $\pi: Y \backslash \pi^{-1}\left(X_{\text {sing }}\right) \rightarrow X \backslash X_{\text {sing }}$ is biholomorphic and such that $\pi^{-1}\left(X \backslash X_{\text {sing }}\right)$ is dense in $Y$. The following was proved by Mumford in [61], by Grauert in [26], and also by Laufer in [45, Theorem 4.4].

Theorem A.10 Let $\pi: Y \rightarrow X$ be a resolution of $X$, and let $p \in X$ be an isolated normal point. Suppose that $A=\pi^{-1}(p)$ is the union of irreducible curves $A_{i}$ which are nonsingular and intersect transversely, with at most two branches of $\bigcup_{i} A_{i}$ passing through any point. Then the intersection matrix

$$
\left(A_{i} \cdot A_{j}\right)
$$

is negative definite.

\section{References}

[1] W Abikoff, Degenerating families of Riemann surfaces, Ann. of Math. (2) 105 (1977) 29-44 MR0442293

[2] $\mathbf{L} \mathbf{V}$ Ahlfors, The complex analytic structure of the space of closed Riemann surfaces., from: "Analytic functions", Princeton Univ. Press, Princton, N.J. (1960) 45-66 MR0124486

[3] J S Athreya, Quantitative recurrence and large deviations for Teichmuller geodesic flow, Geom. Dedicata 119 (2006) 121-140 MR2247652 
[4] W L Baily, Jr, Satake's compactification of $V_{n}$, Amer. J. Math. 80 (1958) 348-364 MR0099451

[5] W L Baily, Jr, On the moduli of Jacobian varieties, Ann. of Math. (2) 71 (1960) 303-314 MR0110715

[6] W L Baily, Jr, On the theory of $\theta$-functions, the moduli of abelian varieties, and the moduli of curves, Ann. of Math. (2) 75 (1962) 342-381 MR0162799

[7] M Bainbridge, Billiards in L-shaped tables with barriers arXiv:0705.3271v1

[8] L Bers, Correction to "Spaces of Riemann surfaces as bounded domains", Bull. Amer. Math. Soc. 67 (1961) 465-466 MR0130972

[9] L Bers, Holomorphic differentials as functions of moduli, Bull. Amer. Math. Soc. 67 (1961) 206-210 MR0122989

[10] L Bers, Fiber spaces over Teichmüller spaces, Acta. Math. 130 (1973) 89-126 MR0430318

[11] L Bers, On spaces of Riemann surfaces with nodes, Bull. Amer. Math. Soc. 80 (1974) 1219-1222 MR0361165

[12] L Bers, Finite-dimensional Teichmüller spaces and generalizations, Bull. Amer. Math. Soc. (N.S.) 5 (1981) 131-172 MR621883

[13] C Birkenhake, H Lange, Complex abelian varieties, volume 302 of Grundlehren der Mathematischen Wissenschaften [Fundamental Principles of Mathematical Sciences], second edition, Springer, Berlin (2004) MR2062673

[14] A I Borevich, I R Shafarevich, Number theory, Translated from the Russian by Newcomb Greenleaf. Pure and Applied Mathematics, Vol. 20, Academic Press, New York (1966) MR0195803

[15] N Bourbaki, Éléments de mathématique. Fasc. XXXV. Livre VI: Intégration. Chapitre IX: Intégration sur les espaces topologiques séparés, Actualités Scientifiques et Industrielles, No. 1343, Hermann, Paris (1969) MR0276436

[16] I Bouw, M Möller, Teichmüller curves, triangle groups, and Lyapunov exponents arXiv:math. AG/0511738

[17] K Calta, Veech surfaces and complete periodicity in genus two, J. Amer. Math. Soc. 17 (2004) 871-908 MR2083470

[18] W Chen, Y Ruan, Orbifold Gromov-Witten theory, from: "Orbifolds in mathematics and physics (Madison, WI, 2001)", Contemp. Math. 310, Amer. Math. Soc., Providence, RI (2002) 25-85 MR1950941

[19] H Cohen, Sums involving the values at negative integers of $L$-functions of quadratic characters, Math. Ann. 217 (1975) 271-285 MR0382192

[20] P Deligne, D Mumford, The irreducibility of the space of curves of given genus, Inst. Hautes Études Sci. Publ. Math. (1969) 75-109 MR0262240 
[21] A Eskin, H Masur, Asymptotic formulas on flat surfaces, Ergodic Theory Dynam. Systems 21 (2001) 443-478 MR1827113

[22] A Eskin, H Masur, M Schmoll, Billiards in rectangles with barriers, Duke Math. J. 118 (2003) 427-463 MR1983037

[23] A Eskin, H Masur, A Zorich, Moduli spaces of abelian differentials: the principal boundary, counting problems, and the Siegel-Veech constants, Publ. Math. Inst. Hautes Études Sci. (2003) 61-179 MR2010740

[24] A Eskin, A Okounkov, Asymptotics of numbers of branched coverings of a torus and volumes of moduli spaces of holomorphic differentials, Invent. Math. 145 (2001) 59-103 MR1839286

[25] A Eskin, A Okounkov, R Pandharipande, The theta characteristic of a branched covering arXiv:math.AG/0312186

[26] H Grauert, Über Modifikationen und exzeptionelle analytische Mengen, Math. Ann. 146 (1962) 331-368 MR0137127

[27] P Griffiths, J Harris, Principles of algebraic geometry, Pure and Applied Mathematics, John Wiley \& Sons, New York (1978) MR507725

[28] R C Gunning, Lectures on complex analytic varieties: The local parametrization theorem, Mathematical Notes, Princeton University Press, Princeton, N.J. (1970) MR0273060

[29] R C Gunning, H Rossi, Analytic functions of several complex variables, Prentice-Hall, Englewood Cliffs, N.J. (1965) MR0180696

[30] E Gutkin, C Judge, Affine mappings of translation surfaces: geometry and arithmetic, Duke Math. J. 103 (2000) 191-213 MR1760625

[31] J Harris, I Morrison, Moduli of curves, Graduate Texts in Mathematics 187, Springer, New York (1998) MR1631825

[32] R Hartshorne, Residues and duality, Lecture Notes in Mathematics 20, Springer, Berlin (1966) MR0222093 Lecture notes of a seminar on the work of A Grothendieck, given at Harvard 1963/64. With an appendix by P Deligne

[33] R Hartshorne, Algebraic geometry, Graduate Texts in Mathematics 52, Springer, New York (1977) MR0463157

[34] F E P Hirzebruch, Hilbert modular surfaces, Enseignement Math. (2) 19 (1973) 183281 MR0393045

[35] P Hubert, S Lelièvre, Prime arithmetic Teichmüller discs in $\mathcal{H}(2)$, Israel J. Math. 151 (2006) 281-321 MR2214127

[36] K Ireland, M Rosen, A classical introduction to modern number theory, second edition, Graduate Texts in Mathematics 84, Springer, New York (1990) MR1070716 
[37] E Kani, The number of curves of genus two with elliptic differentials, J. Reine Angew. Math. 485 (1997) 93-121 MR1442190

[38] E Kani, Hurwitz spaces of genus 2 covers of an elliptic curve, Collect. Math. 54 (2003) 1-51 MR1962943

[39] R Kenyon, J Smillie, Billiards on rational-angled triangles, Comment. Math. Helv. 75 (2000) 65-108 MR1760496

[40] S Kerckhoff, H Masur, J Smillie, Ergodicity of billiard flows and quadratic differentials, Ann. of Math. (2) 124 (1986) 293-311 MR855297

[41] F F Knudsen, D Mumford, The projectivity of the moduli space of stable curves. I. Preliminaries on "det" and "Div", Math. Scand. 39 (1976) 19-55 MR0437541

[42] M Kontsevich, Lyapunov exponents and Hodge theory, from: "The mathematical beauty of physics (Saclay, 1996)", Adv. Ser. Math. Phys. 24, World Sci. Publ., River Edge, NJ (1997) 318-332 MR1490861

[43] M Kontsevich, A Zorich, Connected components of the moduli spaces of Abelian differentials with prescribed singularities, Invent. Math. 153 (2003) 631-678 MR2000471

[44] I Kra, Horocyclic coordinates for Riemann surfaces and moduli spaces. I. Teichmüller and Riemann spaces of Kleinian groups, J. Amer. Math. Soc. 3 (1990) 499-578 MR1049503

[45] H B Laufer, Normal two-dimensional singularities, Annals of Mathematics Studies 71, Princeton University Press, Princeton, N.J. (1971) MR0320365

[46] S Lelièvre, Siegel-Veech constants in H(2), Geom. Topol. 10 (2006) 1157-1172 MR2255494

[47] S Lelièvre, E Royer, Orbitwise countings in $\mathcal{H}(2)$ and quasimodular forms, Int. Math. Res. Not. (2006) Art. ID 42151, 30 MR2233718

[48] A Marden, Geometric complex coordinates for Teichmüller space, from: "Mathematical aspects of string theory (San Diego, CA, 1986)", Adv. Ser. Math. Phys. 1, World Sci. Publishing, Singapore (1987) 341-354 MR915828

[49] H Masur, Interval exchange transformations and measured foliations, Ann. of Math. (2) 115 (1982) 169-200 MR644018

[50] $\mathbf{H}$ Masur, The growth rate of trajectories of a quadratic differential, Ergodic Theory Dynam. Systems 10 (1990) 151-176 MR1053805

[51] H Masur, S Tabachnikov, Rational billiards and flat structures, from: "Handbook of dynamical systems, Vol. 1A", North-Holland, Amsterdam (2002) 1015-1089 MR1928530

[52] C T McMullen, Billiards and Teichmüller curves on Hilbert modular surfaces, J. Amer. Math. Soc. 16 (2003) 857-885 MR1992827 
[53] C T McMullen, Teichmüller geodesics of infinite complexity, Acta Math. 191 (2003) 191-223 MR2051398

[54] C T McMullen, Teichmüller curves in genus two: discriminant and spin, Math. Ann. 333 (2005) 87-130 MR2169830

[55] C T McMullen, Teichmüller curves in genus two: the decagon and beyond, J. Reine Angew. Math. 582 (2005) 173-199 MR2139715

[56] C T McMullen, Teichmüller curves in genus two: torsion divisors and ratios of sines, Invent. Math. 165 (2006) 651-672 MR2242630

[57] C T McMullen, Dynamics of $\mathrm{SL}_{2}(\mathbb{R})$ over moduli space in genus two, Ann. of Math. (2) 165 (2007) 397-456 MR2299738

[58] C T McMullen, Foliations of Hilbert modular surfaces, Amer. J. Math. 129 (2007) 183-215 MR2288740

[59] T Miyake, Modular forms, Springer, Berlin (1989) MR1021004 Translated from the Japanese by Yoshitaka Maeda

[60] M Möller, Periodic points on Veech surfaces and the Mordell-Weil group over a Teichmüller curve, Invent. Math. 165 (2006) 633-649 MR2242629

[61] D Mumford, The topology of normal singularities of an algebraic surface and a criterion for simplicity, Inst. Hautes Études Sci. Publ. Math. (1961) 5-22 MR0153682

[62] D Mumford, The structure of the moduli spaces of curves and Abelian varieties, from: “Actes du Congrès International des Mathématiciens (Nice, 1970), Tome 1”, GauthierVillars, Paris (1971) 457-465 MR0441983

[63] D Mumford, Hirzebruch's proportionality theorem in the noncompact case, Invent. Math. 42 (1977) 239-272 MR471627

[64] D Mumford, Towards an enumerative geometry of the moduli space of curves, from: “Arithmetic and geometry, Vol. II”, Progr. Math. 36, Birkhäuser, Boston (1983) 271-328 MR717614

[65] D Mumford, The red book of varieties and schemes, expanded edition, Lecture Notes in Mathematics 1358, Springer, Berlin (1999) MR1748380 Includes the Michigan lectures (1974) on curves and their Jacobians, With contributions by Enrico Arbarello

[66] Y Namikawa, On the canonical holomorphic map from the moduli space of stable curves to the Igusa monoidal transform, Nagoya Math. J. 52 (1973) 197-259 MR0337981

[67] M Ratner, Invariant measures and orbit closures for unipotent actions on homogeneous spaces, Geom. Funct. Anal. 4 (1994) 236-257 MR1262705

[68] I Satake, On a generalization of the notion of manifold, Proc. Nat. Acad. Sci. U.S.A. 42 (1956) 359-363 MR0079769 
[69] I Satake, On the compactification of the Siegel space, J. Indian Math. Soc. (N.S.) 20 (1956) 259-281 MR0084842

[70] M Schmoll, Spaces of elliptic differentials, from: "Algebraic and topological dynamics", Contemp. Math. 385, Amer. Math. Soc., Providence, RI (2005) 303-320 MR2180242

[71] J-P Serre, Géométrie algébrique et géométrie analytique, Ann. Inst. Fourier, Grenoble 6 (1955-1956) 1-42 MR0082175

[72] J-P Serre, Groupes algébriques et corps de classes, Publications de l'institut de mathématique de l'université de Nancago, VII. Hermann, Paris (1959) MR0103191

[73] CL Siegel, The volume of the fundamental domain for some infinite groups, Trans. Amer. Math. Soc. 39 (1936) 209-218 MR1501843

[74] C L Siegel, Berechnung von Zetafunktionen an ganzzahligen Stellen, Nachr. Akad. Wiss. Göttingen Math.-Phys. Kl. II 1969 (1969) 87-102 MR0252349

[75] G van der Geer, Hilbert modular surfaces, volume 16 of Ergebnisse der Mathematik und ihrer Grenzgebiete (3) [Results in Mathematics and Related Areas (3)], Springer, Berlin (1988) MR930101

[76] W A Veech, The Teichmüller geodesic flow, Ann. of Math. (2) 124 (1986) 441-530 MR866707

[77] W A Veech, Teichmüller curves in moduli space, Eisenstein series and an application to triangular billiards, Invent. Math. 97 (1989) 553-583 MR1005006

[78] W A Veech, Moduli spaces of quadratic differentials, J. Analyse Math. 55 (1990) 117-171 MR1094714

[79] W A Veech, The billiard in a regular polygon, Geom. Funct. Anal. 2 (1992) 341-379 MR1177316

[80] W A Veech, Siegel measures, Ann. of Math. (2) 148 (1998) 895-944 MR1670061

[81] H Whitney, Complex analytic varieties, Addison-Wesley Publishing Co., Reading, MA-London-Don Mills, Ont. (1972) MR0387634

[82] S A Wolpert, On obtaining a positive line bundle from the Weil-Petersson class, Amer. J. Math. 107 (1985) 1485-1507 (1986) MR815769

[83] O Zariski, P Samuel, Commutative algebra. Vol. II, The University Series in Higher Mathematics, D. Van Nostrand Co., Princeton, NJ-Toronto-London-New York (1960) MR0120249

[84] A Zorich, How do the leaves of a closed 1-form wind around a surface?, from: "Pseudoperiodic topology", Amer. Math. Soc. Transl. Ser. 2 197, Amer. Math. Soc., Providence, RI (1999) 135-178 MR1733872

[85] A Zorich, Flat surfaces, from: "Frontiers in number theory, physics, and geometry. I", Springer, Berlin (2006) 437-583 MR2261104 
Department of Mathematics, University of Chicago, 5734 S University Avenue, Chicago, IL 60637, USA

mcb@math.uchicago.edu

Proposed: Shigeyuki Morita

Received: 21 November 2006

Seconded: Benson Farb, Walter Neumann

Accepted: 12 July 2007 